Electrical and Electronics Systems Research Division

\title{
Oak Ridge National Laboratory Annual Progress Report for the Electric Drive Technologies Program
}

Burak Ozpineci, Program Manager

October 2015

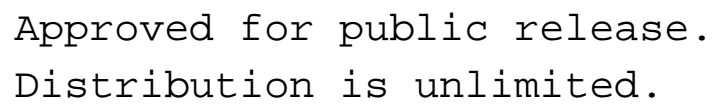




\section{DOCUMENT AVAILABILITY}

Reports produced after January 1, 1996, are generally available free via US Department of Energy (DOE) SciTech Connect.

Website http://www.osti.gov/scitech/

Reports produced before January 1, 1996, may be purchased by members of the public from the following source:

National Technical Information Service

5285 Port Royal Road

Springfield, VA 22161

Telephone 703-605-6000 (1-800-553-6847)

TDD 703-487-4639

Fax 703-605-6900

E-mailinfo@ntis.gov

Website http://www.ntis.gov/help/ordermethods.aspx

Reports are available to DOE employees, DOE contractors, Energy Technology Data Exchange representatives, and International Nuclear Information System representatives from the following source:

Office of Scientific and Technical Information

PO Box 62

Oak Ridge, TN 37831

Telephone 865-576-8401

Fax 865-576-5728

E-mail reports@osti.gov

Website http://www.osti.gov/contact.html

This report was prepared as an account of work sponsored by an agency of the United States Government. Neither the United States Government nor any agency thereof, nor any of their employees, makes any warranty, express or implied, or assumes any legal liability or responsibility for the accuracy, completeness, or usefulness of any information, apparatus, product, or process disclosed, or represents that its use would not infringe privately owned rights. Reference herein to any specific commercial product, process, or service by trade name, trademark, manufacturer, or otherwise, does not necessarily constitute or imply its endorsement, recommendation, or favoring by the United States Government or any agency thereof. The views and opinions of authors expressed herein do not necessarily state or reflect those of the United States Government or any agency thereof. 


\section{Oak Ridge National Laboratory Annual Progress Report for the Electric Drive Technologies Program}

Prepared by:

Oak Ridge National Laboratory

Burak Ozpineci, Program Manager

\section{Submitted to:}

Energy Efficiency and Renewable Energy

Vehicle Technologies Office

Susan A. Rogers, Technology Development Manager

October 2015 

CONTENTS

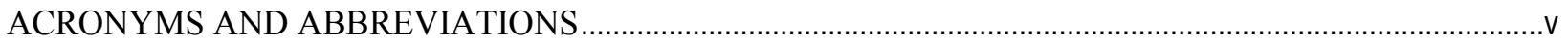

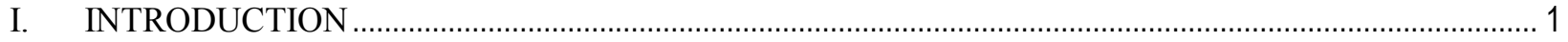

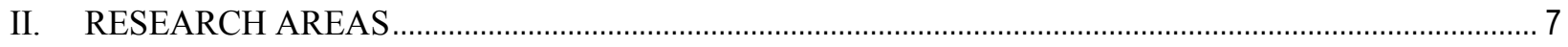

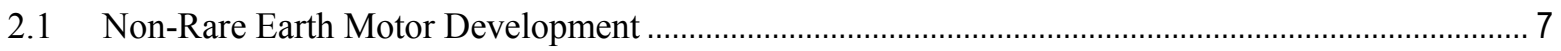

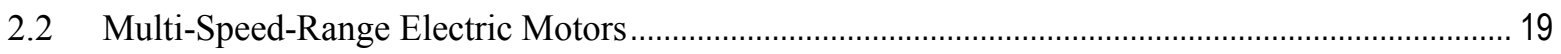

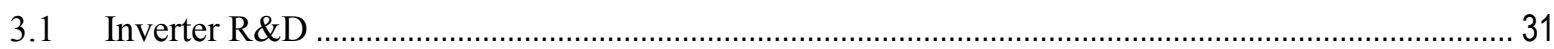

3.2 Innovative Technologies for Converters and Chargers ...............................................................4 43

3.3 Traction Drive Systems with Integrated Wireless Charging ........................................................ 55

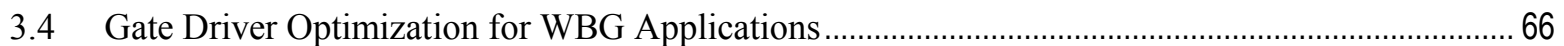

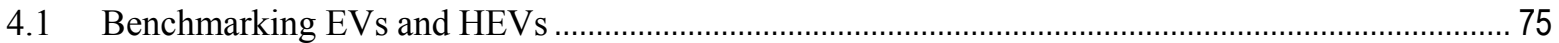

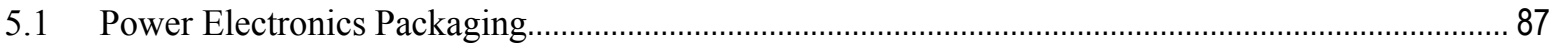

6.1 Power Electronics and Electric Motor Materials Support (Joint with VTO Propulsion Materials) 

ACRONYMS AND ABBREVIATIONS

\begin{tabular}{|c|c|}
\hline $3 \mathrm{D}$ & three dimensional \\
\hline ac & alternating current \\
\hline A & ampere \\
\hline $\mathrm{Ag}$ & silver \\
\hline AGD & active gate drive \\
\hline AM & additive manufacturing \\
\hline $\mathrm{Au}$ & gold \\
\hline BEV & battery electric vehicle \\
\hline C & capacitor \\
\hline $\mathrm{Cl}$ & constant current \\
\hline CTE & coefficient of thermal expansion \\
\hline CV & constant voltage \\
\hline CVD & chemical vapor deposition \\
\hline DBC & direct bond copper \\
\hline dc & direct current \\
\hline $\mathrm{di} / \mathrm{dt}$ & instantaneous current change rate \\
\hline $\mathrm{dv} / \mathrm{dt}$ & instantaneous voltage change rate \\
\hline DOE & US Department of Energy \\
\hline EDT & Electric Drive Technologies \\
\hline EM & electric motor \\
\hline EMI & electromagnetic interference \\
\hline EV & electric vehicle \\
\hline $\mathrm{Fe}$ & iron \\
\hline FEA & finite element analysis \\
\hline GaN & gallium nitride \\
\hline gpm & gallon per minute \\
\hline HEV & hybrid electric vehicle \\
\hline HV & high voltage \\
\hline HV-WBG & high-voltage wide bandgap \\
\hline HWFET & Highway Fuel Economy Test \\
\hline IGBT & insulated gate bipolar transistor \\
\hline JBS & junction barrier Schottky \\
\hline K & degrees Kelvin \\
\hline $\mathrm{L}$ & inductor \\
\hline
\end{tabular}




\begin{tabular}{|c|c|}
\hline MOSFET & metal oxide semiconductor field-effect transistor \\
\hline $\mathrm{MPH}$ & miles per hour \\
\hline MSR & multiple speed range \\
\hline NREL & National Renewable Energy Laboratory (DOE) \\
\hline OBC & onboard charger \\
\hline OEM & original equipment manufacturer \\
\hline ORNL & Oak Ridge National Laboratory \\
\hline PBA & planar bond-all \\
\hline PCB & printed circuit board \\
\hline PE & power electronics \\
\hline PEV & plug-in electric vehicle \\
\hline PFC & power factor correction \\
\hline $\mathrm{Pl}$ & proportional integral \\
\hline PM & permanent magnet \\
\hline PPS & polyphenylene sulfide \\
\hline PWM & pulse width modulated/modulation \\
\hline$R \& D$ & research and development \\
\hline rms & root mean square \\
\hline SBD & Schottky barrier diode \\
\hline $\mathrm{Si}$ & silicon \\
\hline $\mathrm{SiC}$ & silicon carbide \\
\hline SOI & silicon-on-insulator \\
\hline TDS & traction drive system \\
\hline THD & total harmonic distortion \\
\hline UDDS & Urban Dynamometer Driving Schedule \\
\hline US06 & US06 Supplemental Federal Test Procedure \\
\hline U.S. DRIVE & $\begin{array}{l}\text { Driving Research and Innovation for Vehicle efficiency and Energy sustainability (cooperative } \\
\text { research effort between DOE and industry partners) }\end{array}$ \\
\hline V & volt \\
\hline Vac & volts of alternating current \\
\hline Vdc & volts of direct current (operating voltage) \\
\hline VTO & Vehicle Technologies Office (DOE) \\
\hline WBG & wide bandgap \\
\hline ZS & zero sequence \\
\hline ZSIN & zero sequence impedance network \\
\hline
\end{tabular}




\section{INTRODUCTION}

The US Department of Energy (DOE) announced in May 2011 a new cooperative research effort comprising DOE, the US Council for Automotive Research (composed of automakers Ford Motor Company, General Motors Company, and Chrysler Group), Tesla Motors, and representatives of the electric utility and petroleum industries. Known as U.S. DRIVE (Driving Research and Innovation for Vehicle efficiency and Energy sustainability), it represents DOE's commitment to developing public-private partnerships to fund high-riskhigh-reward research into advanced automotive technologies. The new partnership replaces and builds upon the partnership known as FreedomCAR (derived from "Freedom" and "Cooperative Automotive Research") that ran from 2002 through 2010 and the Partnership for a New Generation of Vehicles initiative that ran from 1993 through 2001.

Oak Ridge National Laboratory's (ORNL's) Electric Drive Technologies (EDT) subprogram within the DOE Vehicle Technologies Office (VTO) provides support and guidance for many cutting-edge automotive technologies now under development. Research is focused on developing revolutionary new power electronics (PE), electric motor (EM), and traction drive system (TDS) technologies that will leapfrog current on-the-road technologies, leading to lower cost and better efficiency in transforming battery energy to useful work. The research and development (R\&D) is also aimed at achieving a greater understanding of and improvements in the way the various new components of tomorrow's automobiles will function as a unified system to improve fuel efficiency through research in more efficient TDSs.

In supporting the development of advanced vehicle propulsion systems, the EDT subprogram fosters the development of technologies that will significantly improve efficiency, costs, and fuel economy.

The EDT subprogram supports the efforts of the U.S. DRIVE partnership through a three-phase approach intended to

- identify overall propulsion- and vehicle-related needs by analyzing programmatic goals and reviewing industry recommendations and requirements, and then develop and deliver the appropriate technical targets for systems, subsystems, and component R\&D activities

- develop, test, and validate individual subsystems and components, including EMs and PE

- estimate how well the components and subsystems work together in a vehicle environment or as a complete propulsion system and whether the efficiency and performance targets at the vehicle level have been achieved

The research performed under this subprogram addresses the technical and cost barriers that currently inhibit the introduction of advanced propulsion technologies into hybrid electric vehicles (HEVs), plug-in HEVs, battery electric vehicles (BEVs), and fuel-cell-powered automobiles that meet the goals set by U.S. DRIVE.

A key element in making these advanced vehicles practical is providing an affordable electric TDS. This will require attaining weight, volume, efficiency, and cost targets for the PE and EM subsystems of the TDS. Areas of development include

- novel traction motor designs that result in increased power density and lower cost

- inverter technologies that incorporate advanced wide bandgap (WBG) semiconductor devices to achieve higher efficiency while accommodating higher-temperature environments and delivering higher reliability

- converter concepts that leverage higher-switching-frequency semiconductors, nanocomposite magnetics, higher-temperature capacitors, and novel packaging techniques that integrate more functionality into applications offering reduced size, weight, and cost

- new onboard battery charging electronics that build from advances in converter architectures for decreased cost and size

- more compact and higher-performing thermal controls achieved through novel thermal materials and innovative packaging technologies 
- integrated motor-inverter TDS architectures that optimize the technical strengths of the underlying PE and electric machine subsystems.

ORNL's EDT research program conducts fundamental research, evaluates hardware, and assists in the technical direction of the VTO EDT program and in setting national policy for future BEVs that addresses the overarching goal of petroleum and greenhouse gas reduction. In this role, ORNL serves on the U.S. DRIVE Electrical and Electronics Technical Team, evaluates proposals for DOE, and lends its technological expertise to the direction of projects and evaluation of developing technologies. ORNL also executes specific projects for DOE.

DOE's continuing R\&D into advanced vehicle technologies supports the administration's goal to produce a five-passenger affordable BEV with a payback of less than 5 years and sufficient range and fast charging capability to enable average Americans everywhere to meet their daily transportation needs more conveniently and at lower cost by the year 2022 .

\section{Research Highlights}

\section{Task 2: Electric Motor Research and Technology Development}

\section{Task 2.1 Non-Rare Earth Motor Development}

- Machine design and development

- Developed several non-rare earth motor designs including the use of ferrite magnets.

- Conducted simulations that indicate several of ORNL's motor designs can achieve DOE 2020 targets for power density, specific power, and cost per unit of power.

- Soft magnetic materials R\&D

- Continued research on electrical sheet steel with high silicon $(\mathrm{Si})$ content $(>6 \%)$.

- Confirmed capability for ingot-based processing vs. expensive chemical vapor deposition.

- Developed and confirmed a novel processing technique to reduce brittleness; otherwise, the workability of high-Si steel is not suitable for mass production of motor laminations.

- Advanced modeling

- Began incorporating findings from research on soft magnetic materials properties using the new characterization system into electromagnetics modeling.

- Developed detailed micromagnetics code and a corresponding simulation environment to study the fundamental behavior and impact of various conditions upon the magnetization and loss characteristics of electrical steel.

- Proof-of-principle fabrication and basic testing

- Fabricated a proof-of-principle prototype and started basic testing to confirm preliminary modeling.

\section{Task 2.2 Multi-Speed-Range Electric Motors}

- Confirmed by drive cycle simulation that multiple-speed-range (MSR) operation achieves higher drive cycle efficiency.

- Used a 3-speed-range motor to reduce the total loss by $24 \%$ in a combined drive cycle.

- Developed a novel MSR system design.

- It features fewer added solid-state ac switches and lower losses in low-speed and high-speed mode.

- It does not have a de short-circuit failure mode.

- Conducted a benchtop component test.

- Designed/fabricated a low-cost fast switching ac switch printed circuit board (PCB) assembly for changing switching arrangements.

- Confirmed the capability to decrease current, change winding configurations, and increase current in a short amount of time. 


\section{Task 3: Power Electronics Research and Technology Development}

\section{Task 3.1 Inverter R\&D}

- Completed evaluation of a 1,200 V, 30 A, trench silicon carbide (SiC) metal-oxide-semiconductor field-effect transistor (MOSFET)

- Completed the design, build, and testing of a $10 \mathrm{~kW}$ WBG semiconductor-based air-cooled inverter prototype using ORNL's WBG modules

- Completed the design, build, and testing of a $10 \mathrm{~kW}$ WBG-based liquid-cooled inverter prototype using an advanced package built at ORNL

- Completed the design and build of a $30 \mathrm{~kW}$ WBG-based liquid-cooled inverter

\section{Task 3.2 Innovative Technologies for Converters and Chargers}

- Completed the design, fabrication, and testing of a reconfigurable $\mathrm{SiC}$ traction inverter for use in the development of all-WBG integrated onboard chargers of different topologies. The traction inverter can be configured as either a $100 \mathrm{~kW}$ segmented inverter or a dual 3-phase inverter, each rated at $50 \mathrm{~kW}$.

- Completed the integration and testing of a $6.8 \mathrm{~kW}$ all-SiC integrated onboard charger using the $100 \mathrm{~kW} \mathrm{SiC} \mathrm{segmented} \mathrm{traction} \mathrm{inverter} \mathrm{and} \mathrm{the} \mathrm{isolated} \mathrm{SiC}$ charger dc-dc converter. It showed a peak efficiency of $96.5 \%$ when charged from a $240 \mathrm{~V}$ source and $92.6 \%$ when charged from a $120 \mathrm{~V}$ source, and a 2\% point improvement over the Si-based counterpart developed in FY 2013.

- Completed a design for a $6.8 \mathrm{~kW}$ charger converter using GaN Systems gallium nitride transistors, high-voltage heavy copper PCB power planes, low-voltage (14 V) high-current PCB power planes, and a planar transformer. The design has a high power density of $7.1 \mathrm{~kW} / \mathrm{L}$.

- In collaboration with Aegis Technology, completed fabrication of an advanced magnetic material core set of an E-shaped core and a plate using ORNL's additive manufacturing capability and Aegis Technology's nanocomposite magnetic powders.

\section{Task 3.3 Traction Drive System with Integrated Wireless Charging}

- Simulated various resonant circuits and designed one that is optimized for minimizing circulating current and the associated losses. It shows, with the optimized resonant circuit, a range of $61-75 \%$ reduction in the primary current and $10-44 \%$ reduction in the total losses in a dc-ac wireless charger.

- Simulated and proved concepts for three electric drive topology candidates with integrated wireless charging functionality:

- Topology 1: Tapping into the $14 \mathrm{~V}$ accessory power supply converter to eliminate the secondary ac-dc converter for the wireless charger.

- Topology 2: Using the traction motor and inverter to eliminate the secondary ac-dc converter for the wireless charger

- Topology 3: Using a multiport dc-dc converter that combines a reduced-power boost converter for stepping up the dc bus voltage of the traction drive inverter, a $14 \mathrm{~V}$ buck converter for powering the $14 \mathrm{~V}$ vehicle accessory loads, and a wireless battery charging converter.

- Simulation results show all the wireless chargers have high input power factors of greater than $99 \%$ and low total harmonic distortion factors of less than $3.5 \%$ in the ac source current.

\section{Task 3.4 Gate Driver Optimization for WBG Applications}

- Research of Existing Methods

- Conducted a review of the scientific literature dealing with state-of-the-art methods of performing $\mathrm{di} / \mathrm{dt}$ and $\mathrm{dv} / \mathrm{dt}$ control. Three primary protection methods were identified: solid state circuit breaker, fault current evaluation, and desaturation detection. Of these, desaturation detection was chosen to be the most appropriate, particularly for approaches with a goal of maximized monolithic integration. 
- Of the active gate drive methods identified, chose source inductance-based current measurement. All methods addressed Si devices (insulated gate bipolar transistors and MOSFETs), with none addressing the specific needs of driving $\mathrm{SiC}$ devices. Furthermore, all methods appeared monolithically compatible, but none was found that had been implemented as an integrated circuit or integrated circuit chipset.

- Established di/dt as the most appropriate measured parameter from this literature search and reviewed conventional means of current measurement, specifically for measuring the drain current of a power MOSFET.

- Determined current measurement using a sense inductor to be the overall best choice for motor drive applications.

- Gate Driver Design and Architecture Selection

- Investigated multiple gate-drive waveforms and carried out simulations using SPICE to predict efficacy in controlling the $\mathrm{di} / \mathrm{dt}$ and $\mathrm{dv} / \mathrm{dt}$ of a MOSFET switching a motor leg.

- Constructed a double pulse test setup and used it to evaluate the transient switching characteristics of commercially available SiC MOSFET devices from Cree. Using data from these tests, some SPICE model adjustments were made and simulations re-run.

- Determined that improved SPICE models were needed for more accurate dynamic simulation of the WBG devices.

- To address the SiC MOSFET modeling need, identified a private company with expertise in SiC circuit modeling and simulation. A commercial simulation tool (CoolSPICE) was acquired, along with a few $\mathrm{SiC}$ powerMOS device models for evaluation.

- Gate Driver Design Simulations

- Developed a new gate drive waveform (variable threshold) and showed via simulation that it provides the desired control of the transient switching characteristics. This method also automatically compensates for variation in the powerMOS threshold voltage from device to device and over temperature.

- Performed simulations of the closed-loop gate driver in PSPICE using vendor-supplied SiC powerMOS device models (C2M0080120D, Cree Inc.). The loop control set point was stepped to demonstrate the range of operation. Some simplifications were used to improve the simulation convergence, including the use of a single-slope gate drive waveform. Results showed the loop operated in a stable fashion.

- Began enhanced modeling activity with CoolSPICE software. It will be carried forward in year 2 of the project.

\section{Task 4: Benchmarking, Testing, and Analysis}

\section{Task 4.1 Benchmarking EV and HEVs}

- Designed, fabricated, and assembled the hardware necessary to adapt the 2014 Honda Accord HEV transmission to the dynamometer test cell.

- Successfully implemented the integration of ORNL controls with the 2014 Accord power converter unit.

- Conducted comprehensive dynamometer testing of a 2014 Accord inverter and motor at 300, 500, and $700 \mathrm{Vdc}$ to obtain an efficiency map and many other performance metrics.

- Confirmed published peak torque and power specifications for a 2014 Accord inverter and motor.

\section{Task 5: Packaging Research and Technology Development}

\section{Task 5.1 Power Electronics Packaging}

- Manufactured and delivered high-power SiC power modules for air-cooling system evaluation that allow a reduction of $30 \%$ in overall volume and weight.

- Demonstrated that integrated cooled planar SiC power electronics modules not only increase power density by $60 \%$ but also enable a threefold increase in current density over their conventional $\mathrm{Si}$ 
counterparts, resulting from a $35 \%$ reduction in the die size; 40 and $80 \%$ reductions in conduction and switching power losses, respectively; and a $35 \%$ reduction in package thermal resistance.

- Fabricated and delivered sintered-silver (Ag) die-attached $\mathrm{SiC}$ power modules. Initiated research on that technology to develop high-reliability, high-temperature WBG power electronics.

\section{Task 6: Other Support}

\section{Task 6.1 Power Electronics and Electric Motor Materials Support}

- Power electronics support

- Quantified the shear strength of sintered-Ag interconnects as a function of printing method (screen vs. stencil printing) and plating material. Initiated failure analysis to censor the measured strengths in the context of cohesive and adhesive failure locations. These results will illustrate the achievable strength characteristics of such interconnect systems, guide future sintered-Ag design for power electronics, and improve the community's receptiveness to adopting sintered-Ag technology.

- Conceived of, coordinated, fabricated all specimens for, and assumed leadership of an ORNL-led collaboration with National Renewable Energy Laboratory (NREL) to study delamination initiation and propagation response in sintered-Ag interconnects.

- Sectioned and analyzed the micro-architecture of the power module used in the Honda Accord inverter in support of EDT Task 4.1.

- Used sintered $\mathrm{Ag}$ as an interconnect to bond a SiC die to direct-bonded copper substrates in support of EDT Task 5.1.

- Fabricated sintered-Ag samples for thermal and mechanical testing at NREL.

- Electric machines support (EDT Task 2.1)

- Measured thermal properties of developmental, mineral-filled potting and molding compounds and established a collaboration with an industrial manufacturer of such materials.

- Conceived of, coordinated, fabricated all specimens for, and assumed leadership of an ORNL-led collaboration with NREL to better understand the directional thermal response of copper-wound structures.

- Developed versatile copper-wound billets from which samples were harvested to enable the thermal property measurement of their anisotropic structure using three different standardized techniques.

- Showed that the thermal conductivity in the direction of the copper wires is over two orders of magnitude higher than the thermal conductivity perpendicular to them. 



\section{RESEARCH AREAS}

\subsection{Non-Rare Earth Motor Development}

Tim Burress, Principal Investigator

Oak Ridge National Laboratory (ORNL)

National Transportation Research Center

2360 Cherahala Boulevard

Knoxville, TN 37932

Phone: (865) 946-1216

E-mail: burressta@ornl.gov

\section{Susan A. Rogers, DOE EDT Program Manager}

Phone: (202) 586-8997

E-mail: Susan.Rogers@ee.doe.gov

\section{Burak Ozpineci, ORNL EDT Program Manager}

Phone: (865) 946-1329

E-mail: burak@ornl.gov

Contractor: UT-Battelle, LLC, managing and operating contractor for the Oak Ridge National Laboratory Contract No.: DE-AC05-00OR22725

\section{Abstract/Executive Summary}

Almost all hybrid electric vehicles (HEVs) and electric vehicles (EVs) use permanent magnets (PMs) with rare earth materials such as neodymium and dysprosium because they facilitate the achievement of high power densities, specific powers, and efficiencies. However, there has been significant market volatility associated with these rare earth materials in recent years, including a price increase for dysprosium by a factor of 40 within one year. Therefore, alternatives to rare earth PM motors are of very high interest to original equipment manufacturers and suppliers, particularly as vehicles become more electrified and uses for rare earth materials in other applications expand. Achieving competitive performance and efficiency with alternative motors having comparable mass, volume, voltage, and other key metrics requires a highly advanced multidisciplinary research approach including high-accuracy modeling; the research, use, and development of soft and hard magnetic materials; and novel multi-objective nonlinear optimization computational design methods.

\section{Accomplishments}

- Machine design and development

- Developed several non-rare earth motor designs including the use of ferrite magnets.

- Conducted simulations that indicate several of ORNL's motor designs can achieve DOE 2020 targets for power density, specific power, and cost per unit of power.

- Soft magnetic materials research and development (R\&D)

- Continued research on electrical sheet steel with high silicon ( $\mathrm{Si})$ content $(>6 \%)$.

- Confirmed capability for ingot-based processing vs. expensive chemical vapor deposition (CVD).

- Developed and confirmed a novel processing technique to reduce brittleness; otherwise, the workability of high-Si steel is not suitable for mass production of motor laminations.

- Advanced modeling

- Began incorporating findings from research on soft magnetic materials properties using the new characterization system into electromagnetics modeling.

- Developed detailed micromagnetics code and a corresponding simulation environment to study the fundamental behavior and impact of various conditions upon the magnetization and loss characteristics of electrical steel. 
- Proof-of-principle fabrication and basic testing

- Fabricated a proof-of-principle prototype and started basic testing to confirm preliminary modeling.

\section{Introduction}

As the electric motor is one of the main components of HEV and EV drivetrains, improving efficiency, performance, and cost-effectiveness is crucial to the hybridization and electrification of vehicles. Because PM motors are not easily surpassed in terms of efficiency, power density, and specific power, almost all HEVs and EVs use them. However, the cost of rare earth PM material accounts for at least $40 \%$ of the entire motor cost; and the high and unstable costs of rare earth materials are causing automotive manufacturers and suppliers around the world to seek alternative non-rare earth motor technologies that facilitate cost effectiveness as HEV and EV markets expand. Therefore, the development of alternative non-rare earth motor technologies plays an important role in the future interests of economic stability, clean energy, and energy independence.

\section{Approach}

The primary objective of ORNL's motor R\&D is to develop low-cost non-rare earth motor solutions with high power density, specific power, and efficiency. The overall structure of the efforts in the project is described by the diagram in Figure 1. Key efforts of the project include conventional motor design techniques, advanced motor modeling, motor materials research, and empirical verification.

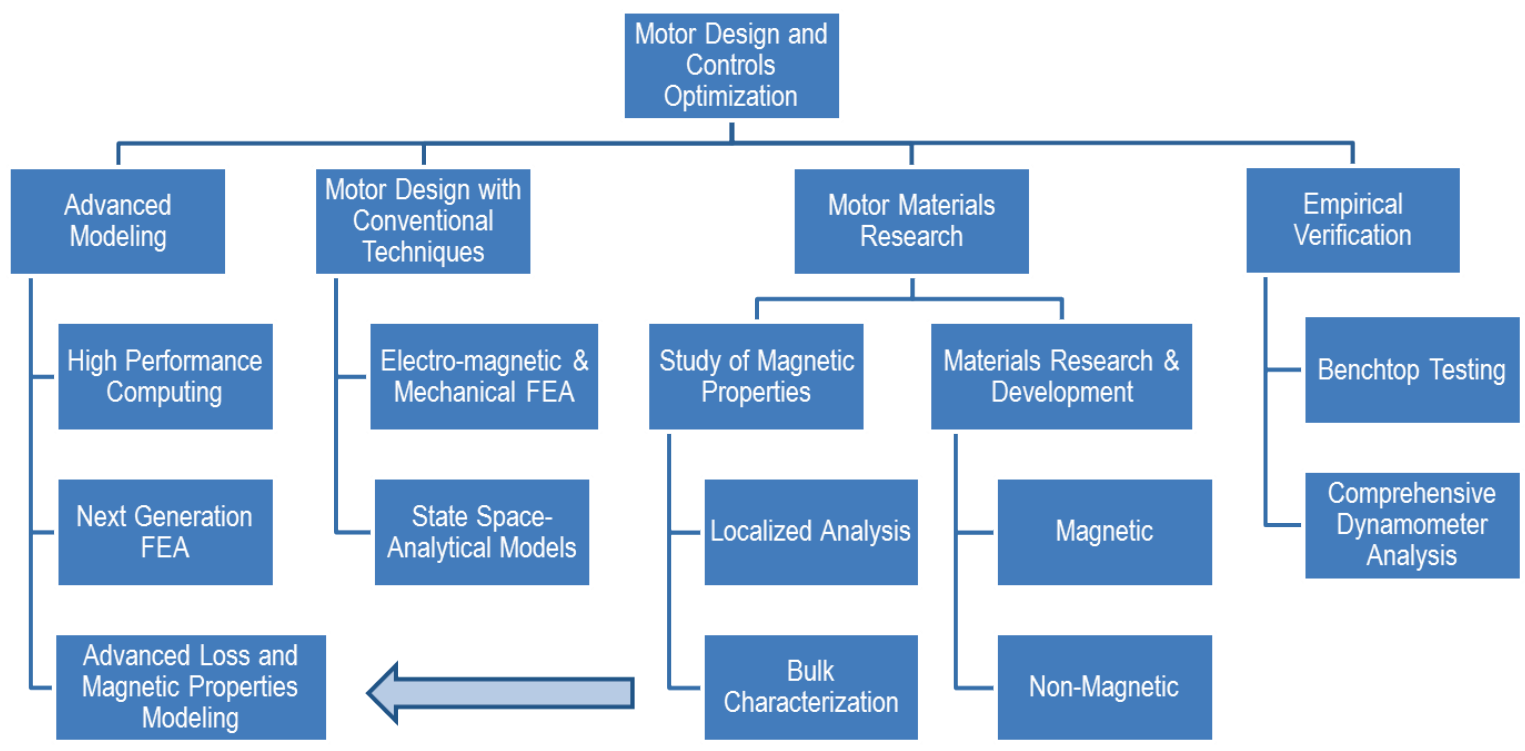

Figure 1: Various exterior views of the $\mathbf{2 0 1 4}$ Honda Accord hybrid power converter unit.

In the initial stages of this project, many types of novel electric motor designs were developed and considered in coarse simulations to identify the most feasible designs that have high potential for commercialization. The wide range of motor types studied includes field excitation, synchronous reluctance, and non-rare earth PM motors, and combinations of these machine types. Proof-of-principle components were built and tested to serve as a feedback mechanism for novel motor development and fundamental soft magnetic materials research. Novel motor designs were developed and optimized using high-performance computing techniques, beginning with implementation and modeling on parallelized workstations. Preparations are under way for the utilization of computational clusters and ultimately the supercomputer at the computational facility at ORNL. Findings from fundamental magnetic materials research are being incorporated into new modeling tools to improve accuracy, and this is greatly facilitated with parallelized computing. 
In addition to novel motor and controls development, the development and use of new materials and materials processing techniques is under way to improve the magnetization and/or efficiency characteristics of soft magnetic materials (e.g., electrical steel). This effort is being conducted in coordination with the VTO Propulsion Materials Program. Additionally, new materials or manufacturing techniques are being studied and used to achieve improvements in manufacturability (cost reduction), cooling (in collaboration with National Renewable Energy Laboratory), and improved mechanical integrity.

To improve motor modeling accuracy and complement new materials and processing developments, fundamental research is being conducted to study the phenomena behind magnetization and loss characteristics of soft magnetic materials in electric motors, details that are still not fully quantified or understood in the scientific community. For example, residual stresses or induced stresses incurred during manufacturing or motor operation have a considerable impact upon losses and permeability. Additionally, the impact of temperature, pulsed-width modulation excitation, and other factors impact the magnetic properties in a way that is not considered or modeled with conventional finite element analysis (FEA) tools. These studies support the development of high-fidelity models and modeling tools, which are particularly important as highperformance computing is used in the optimization of detailed geometric features to achieve high power density, specific power, efficiency, and cost effectiveness.

\section{Results and Discussion}

Motor design optimization in FY 2015 was conducted with commercial FEA packages in combination with other software packages for parametric optimization algorithms as well as state space simulations. The progress and results in the area of motor design are discussed after the following sections describing the parallel efforts in materials R\&D. These parallel efforts are ultimately in support of improving motor designs by developing or facilitating the use of more efficient materials and by conducting fundamental research to improve the modeling accuracy of electrical steel characteristics. Findings from this research will continue to be incorporated into new modeling tools in FY 2016.

\section{Motor Materials Research-6.5\% Silicon Steel}

ORNL is developing ways to facilitate the use of electrical steel with high Si content. Near the end of FY 2014, this effort began to be a joint project with the VTO Propulsion Materials Program. Conventional electrical steel has about 2-3\% Si content, and there are some expensive products on the market with $6.5 \% \mathrm{Si}$ content. A higher amount of $\mathrm{Si}$ increases resistivity and therefore reduces eddy current and hysteresis losses. The comparison in Figure 2 indicates that an increase from 3 to $6.5 \%$ Si yields an average core loss reduction of about $35 \%$. This reduction of core loss is nearly indirectly proportional to the resistance increase; thus the specific resistance of the 3\% Si steel (labeled "Non-oriented $\mathrm{SiFe")} \mathrm{is} \mathrm{about} 44 \%$ lower than the specific resistance of $6.5 \% \mathrm{Si}$ (labeled "JNEX-Core"). The loss reduction is consistent for the various frequencies and flux density levels indicated.

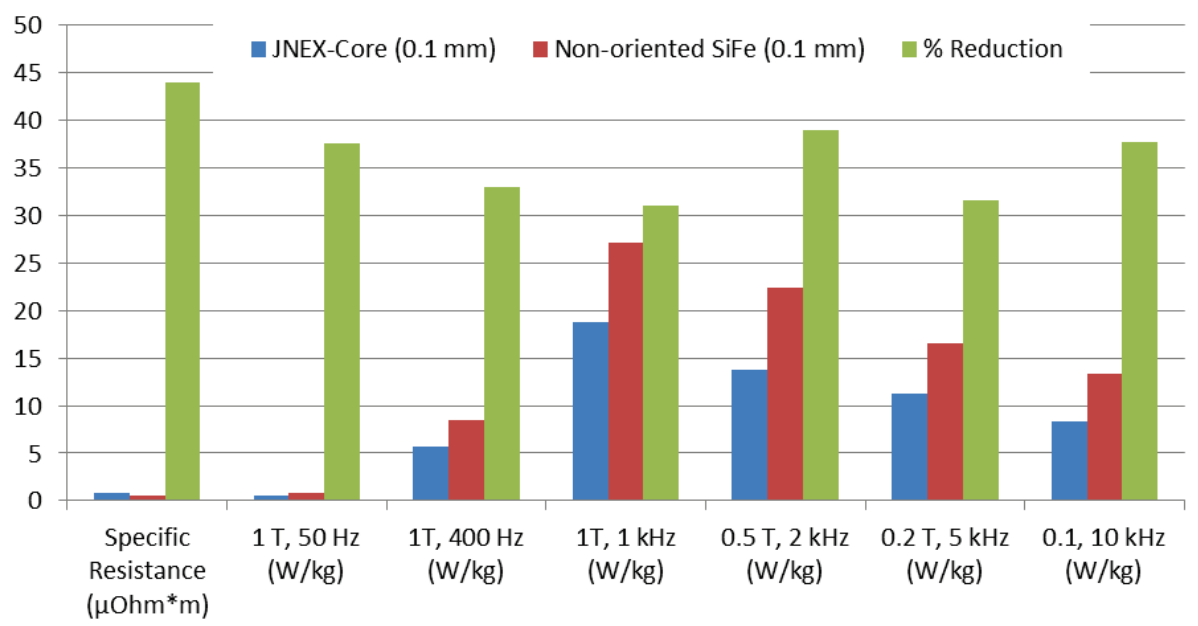

Figure 2: Comparison of electrical steel core losses: $6.5 \% \mathrm{Si}$ vs $3 \% \mathrm{Si}$. 
Currently, $6.5 \% \mathrm{Si}$ is commercially available, but it is expensive because it is made using an intensive CVD process. Silicon steel is rolled into sheets about 0.1 to $1 \mathrm{~mm}$ thick, depending on the application requirements. The CVD process is used because conventional $6.5 \% \mathrm{Si}$ steel is so brittle that it cannot be easily rolled and mechanically worked with conventional methods. Therefore, $6.5 \% \mathrm{Si}$ sheet processing begins with $3 \% \mathrm{Si}$ sheets, and the CVD process is used to increase the Si content to $6.5 \%$. It remains a challenge to produce motor laminations from conventional $6.5 \% \mathrm{Si}$ sheet steel, since a stamping process is used for mass-produced motors. The brittle $6.5 \% \mathrm{Si}$ sheet steel is difficult to stamp consistently without undesirable fracturing, and stamping tool lifetime is reduced because the material is harder.

Research on this project aims to facilitate the production of $6.5 \%$ Si sheet steel from ingot form using themomechanical processing techniques that are similar to conventional methods. Trace elements can be added to enhance strain softening behavior. In previous years, this phenomenon was confirmed with experimental measurements as samples were prepared with trace amounts of various materials, including boron. Results confirmed that ductility can be recovered during warm rolling, in which certain crystalline phase ordering is destroyed.

In addition to developing methods to produce $6.5 \% \mathrm{Si}$ from ingots, ORNL is developing processing techniques to facilitate the rolling process, as well as lamination production. This is a critical part of the development, because lamination quality control and tool lifetime are key considerations for motor production. Hardness measurements confirm that ORNL's new processing method softens $6.5 \% \mathrm{Si}$ to a level similar to the softness of 3\% Si without significant modification of conventional processing methods. The hardness and brittleness is associated with the formation of B2 ordered domains in the steel microstructure when Si content exceeds a critical amount, and this precludes cold rolling of the steel. Recently, it has been shown that warm rolling the steel to a critical strain results in the destruction of the B2 order because of the dislocation flow that renders the steel ductile enough for subsequent cold rolling to the finish thickness. Such an approach is clearly less expensive than the current process based on diffusion annealing.

In FY 2015, we performed thermomechanical processing of Fe-Si-B steels with different Si and boron contents. The steels possessed sufficient ductility during warm rolling that significant thickness reductions could be achieved without incidence of cracking. The microstructure after warm rolling indicated reduced B2 ordering. However, the dislocation density after warm rolling was high enough to adversely impact the roomtemperature ductility. We are currently performing controlled warm deformation studies to optimize the warm rolling conditions that would maximize the subsequent room-temperature ductility for cold rolling of the sheet to the finish thickness.

Significant progress has also been made in investigating mechanisms that would result in significant softening of the steel during secondary forming operations, thus potentially reducing die wear. As part of the effort to understand the fundamental mechanisms that result in the destruction of the B2 order, we performed molecular dynamics simulations of warm deformation using a modified embedded atom potential (MEAM) for $\mathrm{Fe}-\mathrm{Si}$, shown in Figure 3. The initial microstructure consisted of multiple B2 domains in the form of circular discs lying in the [100] planes. These simulations were able to capture the reduction in the B2 order due to deformation consistent with experimental observation. The B2 domains were broken up to smaller and smaller sizes with increasing deformation. The experimental effort is continuing with the additional melting and casting of additional $\mathrm{Fe}-\mathrm{Si}-\mathrm{B}$ alloy compositions for subsequent thermomechanical processing trials in FY 2016.
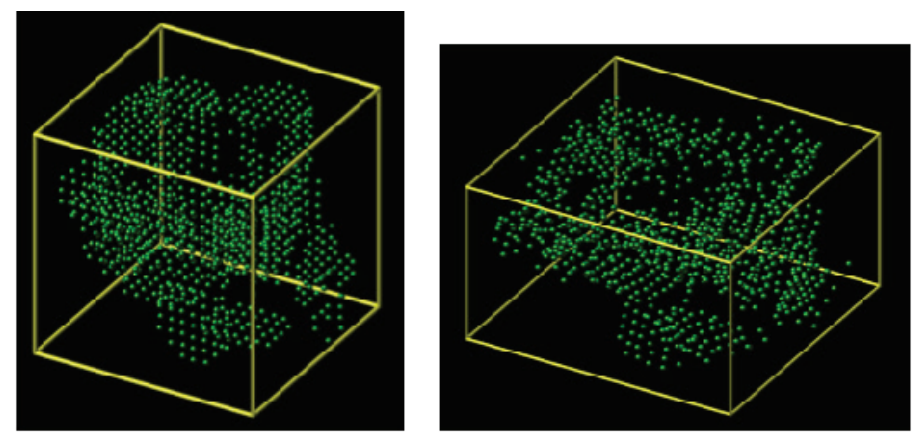

Figure 3: Simulated Si positions before and after a compressive strain of 0.153 . 


\section{Motor Materials Research—Electrical Steel Modeling}

The objective of this effort is to simulate the domain structure in iron $(\mathrm{Fe})$ and $\mathrm{Fe}-\mathrm{Si}$ alloys and the evolution of the domain structure during magnetization. The simulations will also capture the effect of nonmagnetic inclusions and elastic strain fields on the domain wall velocity and its effect on the magnetization curves. The output of the simulations will be used to improve finite element simulations of the electric motor to provide physically based constitutive laws for the soft magnetic materials used. The simulations use a Monte Carlo technique to evolve the distribution of the magnetic moments in the material, taking into account energy contributions from external magnetizing field, exchange energy, magneto-crystalline anisotropy, and magnetic dipole-dipole interaction. A message passing interface (MPI) -based parallel code has been developed that calculates exchange energy using up to $3^{\text {rd }}$-nearest neighbors, and calculates the dipole-dipole interactions through a fast multi-pole technique. A scaling law has been developed for the exchange energy term when the simulations are scaled from an atomistic to a mesoscopic length scale where each atom is replaced by a collection of unit cells.

The simulations have been used to simulate the stable presence of a domain wall when the simulation size exceeds the single-domain particle size for iron. The single-crystal simulations have been used to quantify the drag force exerted on a strain domain wall owing to the presence of a non-magnetic inclusion. The magnetization curves for a single crystal with a cube orientation and a crystal with a random orientation were generated using the simulation approach that captures the hysteresis loop associated with demagnetization and re-magnetization. The simulations have been extended to polycrystals, where the orientation of each grain in the polycrystal is identified by the three Euler angles. A realistic, three-dimensional polycrystalline microstructure was simulated using Potts model. The simulations were able to capture the effects of grain orientations and of the evolution of magnetic moments in each grain on the overall hysteresis of the microstructure. In all of the above domain wall simulations, a theoretical material with uniaxial magnetocrystalline anisotropy was used. The magnitude of the magneto-crystalline anisotropy energy relative to the exchange energy was artificially increased to obtain a stable magnetic domain wall within a relatively small computational volume. A director's discretionary project has been requested in order to perform the simulations using Titan, ORNL's leadership-class supercomputer, so that the above constraint on the simulation size can be relaxed and the simulations can be performed for realistic materials, including Fe and Fe-Si based magnetic materials.

Figure 4 shows the simulation results for the magnetization of a randomly oriented single crystal. The initial stable structure contains a few domains. Initial magnetization involves the growth of the domain, which is closest to the applied direction at the expense of the other unfavorably oriented domains. Subsequent magnetization involves the rotation of the magnetic moments in the single domain to the magnetization direction. During the demagnetizing stage as well as the re-magnetizing stage, the single domain remains intact and the magnetic moments rotate to reduce the net magnetization in the direction of the applied field. One of the aspects that the simulation is missing is the rotation of a collection of spins to the easy magnetization directions to nucleate the domains during the demagnetization stage. The Monte Carlo code is being improved to include cluster Monte Carlo moves and implement it in a parallel code to overcome this challenge. 


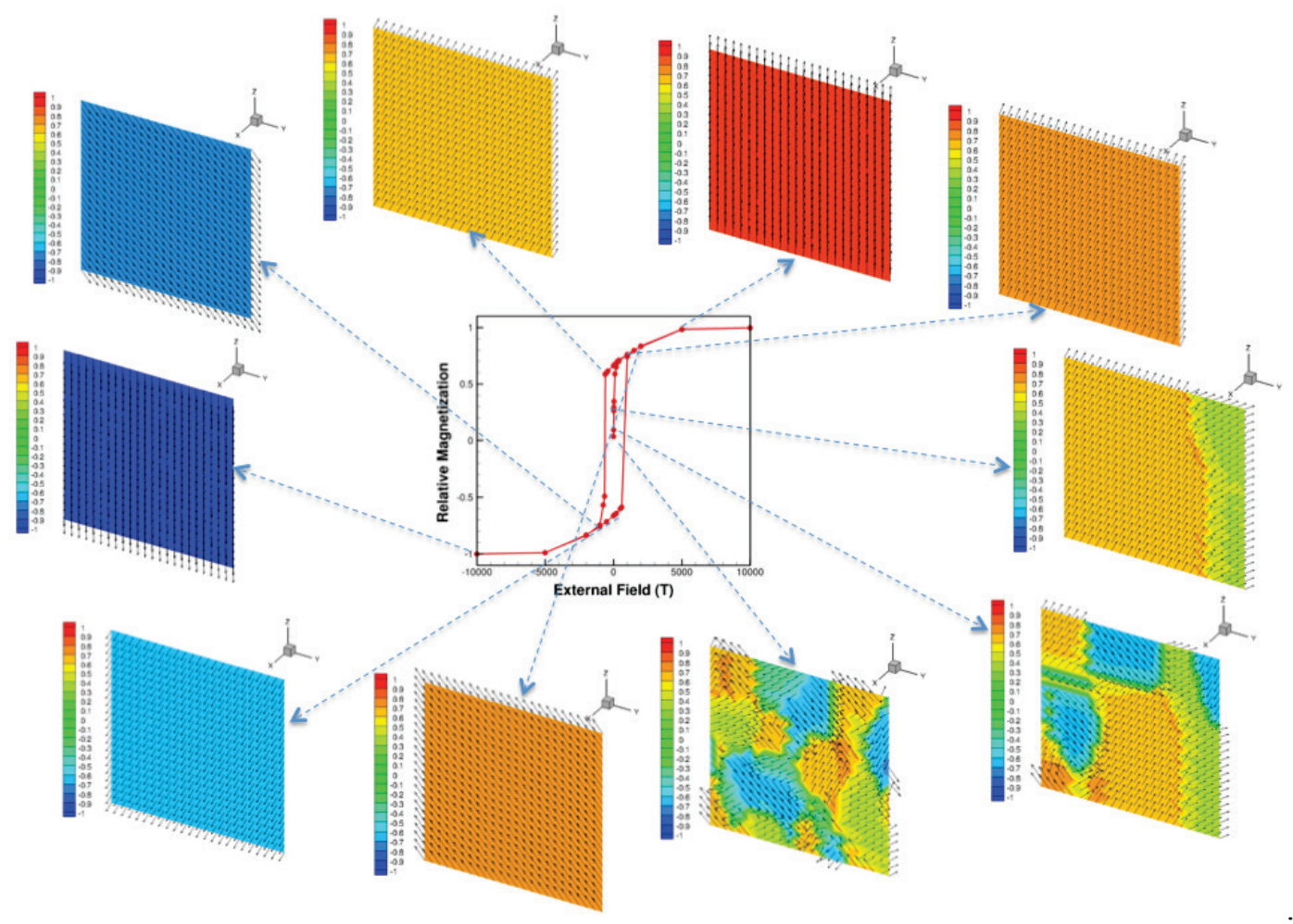

Figure 4: Monte Carlo simulation of the hysteresis loop for a randomly oriented single crystal showing the evolution of the initial domain structure.

\section{Motor Design and Modeling}

The development of unconventional motor technologies was accomplished by improvising upon promising technologies from previous work and initiating work on novel motor concepts. Previous research includes the design of various motor types, including the induction motor, switched reluctance motor, hybrid excitation motor, and flux coupling/field coil machines, with the latter two displaying the most potential. Novel motor designs were conceived and simulated, with consideration of various machine types such as switched reluctance, hybrid PM excitation, synchronous reluctance, and field coil excitation. Recent designs include the utilization of ferrite magnets for improved power density.

ORNL used advanced optimization algorithms to optimize motor design and controls. Electromagnetic FEA tools were used to implement basic models to determine the performance and operational characteristics of new motor designs. Analytical models were used to determine additional performance metrics and associated control methods.

\section{Considerations in Using Non-Rare Earth Permanent Magnets}

Non-rare earth PM motors must accommodate a much larger volume of magnet material in the rotor to achieve similar performance to that of a motor employing rare-earth materials. There are two main reasons for this. First, non-rare earth magnets, such as ferrites, have a much lower remanent flux density than those based on rare earths like $\mathrm{NdFeB}$. For high-performance ferrites, a typical remanent flux density is about $0.4 \mathrm{~T}(4 \mathrm{kG})$ whereas $1.2 \mathrm{~T}(12 \mathrm{kG})$ is typical for $\mathrm{NdFeB}$ magnets. This indicates that the total surface area of ferrite magnets in an electric motor rotor must be three times greater than in an $\mathrm{NdFeB}$ motor producing an equivalent flux-linkage in the stator.

Second, ferrites have a much lower intrinsic coercivity than $\mathrm{NdFeB}$ magnets. For example, the Hitachi Metals 12 series ferrites have coercivities in the range of 300-450 kA/m (3.8-5.7 kOe). In comparison, the coercivity of $\mathrm{NdFeB}$ magnets will be closer to $950 \mathrm{kA} / \mathrm{m}(12 \mathrm{kOe})$. Again, the ratio is about 3:1. Therefore, to resist demagnetization against a field produced by a given number of amp-turns in the stator, the magnets in a ferrite 
design would need to be about three times as thick as an equivalent design with rare earth materials. It should be emphasized that this approximation assumes similar stator designs for both ferrite and NdFeB designs. This issue will be addressed in more depth later.

Depending on the effective magnetic path length through the motor, each PM material has an associated magnet aspect ratio that operates the magnet at maximum flux per volume. Achieving high flux levels leads to increasingly poor magnet utilization and lower torque/power per dollar. Rare earth motors can be designed more economically by using reluctance torque to achieve the targeted torque and power levels. For example, about half of the 2010 Toyota Prius's peak torque is due to reluctance torque. On the other hand, motors that do not operate with high reluctance torque have a potential benefit of an improved power factor.

Stators with concentrated windings have several advantages, as the teeth can be wound individually and a higher slot fill factor can be achieved. The short end turn length reduces the volume of non-active material in the motor (from a mechanical power production standpoint). Both of these factors lead to an overall decrease in copper losses.

A disadvantage of concentrated windings is that they can cause time-varying fields in the rotor, increasing rotor core losses. This is an especially serious issue for rotors with rare earth PMs, which have high conductivities. Any time-varying fields in the rotor will induce eddy currents in the PMs which will cause the PM to heat up, decreasing performance and increasing the risk of permanent demagnetization. This is less an issue for ferrite magnets because of their low conductivity and good temperature stability.

\section{Design Constraints and Concentrated Windings}

The concentrated winding design allows for the balancing of core losses and time-varying rotor fields with a corresponding decrease in copper losses. From a heat transfer perspective, losses occurring in the core of the stator often sustain a higher effective rate of heat transfer to the outside of the motor. In contrast, heat generated in the windings must be transferred through insulating materials to the stator core and is more difficult to manage.

To assess the feasibility of a concentrated winding motor design within a practical application framework, we have imposed constraints on our design based on the existing hybrid powertrain topology of the 2010 Toyota Prius. In particular, the voltage limit, current limit, stator outer diameter, and maximum speed were taken directly from this system.

For motors with no appreciable reluctance torque, the torque $\tau$ is given by $\tau=1.5 N_{p} N_{t} \bar{\lambda}_{p m} i_{q}$ where $N_{p}$ is the number of motor poles, $N_{t}$ is the number of turns, $i_{q}$ is the quadrature current, and $\bar{\lambda}_{p m}$ is the normalized (single-turn) PM flux-linkage. Increasing the torque by achieving a large number of turns is limited by (1) the risk of demagnetizing the PM materials and (2) saturation of the magnetic core. From a bulk parameter perspective, the demagnetization risk is associated purely with a direct axis current $\left(i_{d}\right)$, which is not the torque-producing component. At the continuum level, this is largely true for PM motors with integer slot winding configurations because, for each pole, the field generated by $i_{q}$ is truly in quadrature with the PM fields.

The situation is different for concentrated windings that produce a spatial distribution of the flux with rather large subharmonics. The bulk parameter model is the result of the phasor sum of the individual stator teeth interacting with the rotor poles, and the effects of the subharmonics are unobservable in this model. In the worst-case scenario, the peak demagnetizing field is produced by two adjacent teeth roughly aligned with different rotor poles. This is to say a PM motor with concentrated windings must be protected against demagnetization from both direct and quadrature currents. This depends on the tooth/winding configuration, with some choices increasing the risk of q-axis demagnetization more than others, as demonstrated in Figure 5.

The other limiting factor is saturation of the core. An ideally sized motor would meet the demagnetization and saturation constraints simultaneously. A saturation limitation would reduce the ability to perform field weakening in order to achieve constant power over a wide speed range. Alternatively, it implies an overuse of PM material. The saturation flux density can be improved through choice of material, but this quantity usually varies inversely with the core losses for materials of similar cost. 


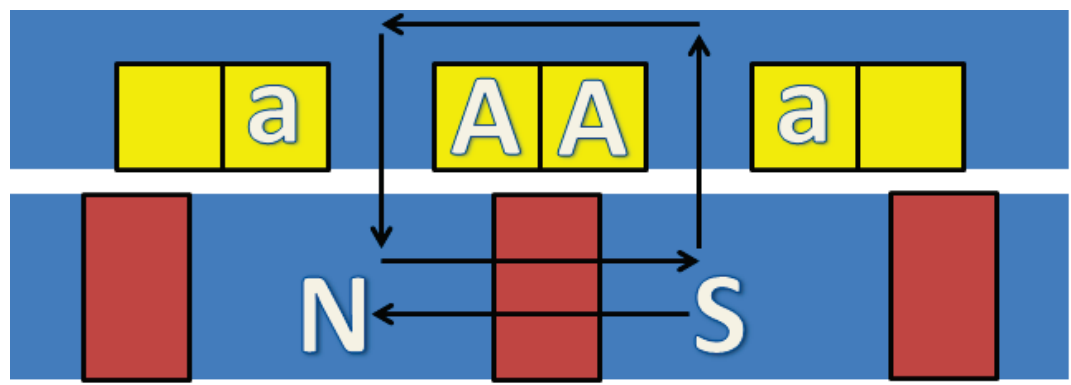

Figure 5: Certain concentrated winding configurations contain two adjacent phases with opposite polarities. While possessing certain favorable characteristics in terms of torque ripple and back-electromotive-force, they can pose a significant risk of demagnetization with a pure quadrature axis current.

\section{Design A}

Rough analytical models led to the development of a refined ferrite motor, referred to as Design A. An electromagnetic efficiency map for this design is shown in Figure 6. Note that this does not include mechanical or inverter losses. The peak torque of the motor is $140 \mathrm{~N}-\mathrm{m}$ and the peak power output is $80 \mathrm{~kW}$ above $6,000 \mathrm{rpm}$. This initial design demonstrated that it is possible to achieve significant torque and power capabilities using only ferrite magnets in this configuration. This particular design, however, had several flaws. First, concerns about demagnetizing the PMs limited the torque to less than what would otherwise be achievable within the saturation limit of the magnetic circuit. Second, the particular winding configuration produced reluctance torque that opposed the PM torque. This ultimately decreased the torque/power output by about $10 \%$. Third, the analytical equations used for the initial sizing made geometric approximations that were less than ideal in estimating the magnet arrangement and size.

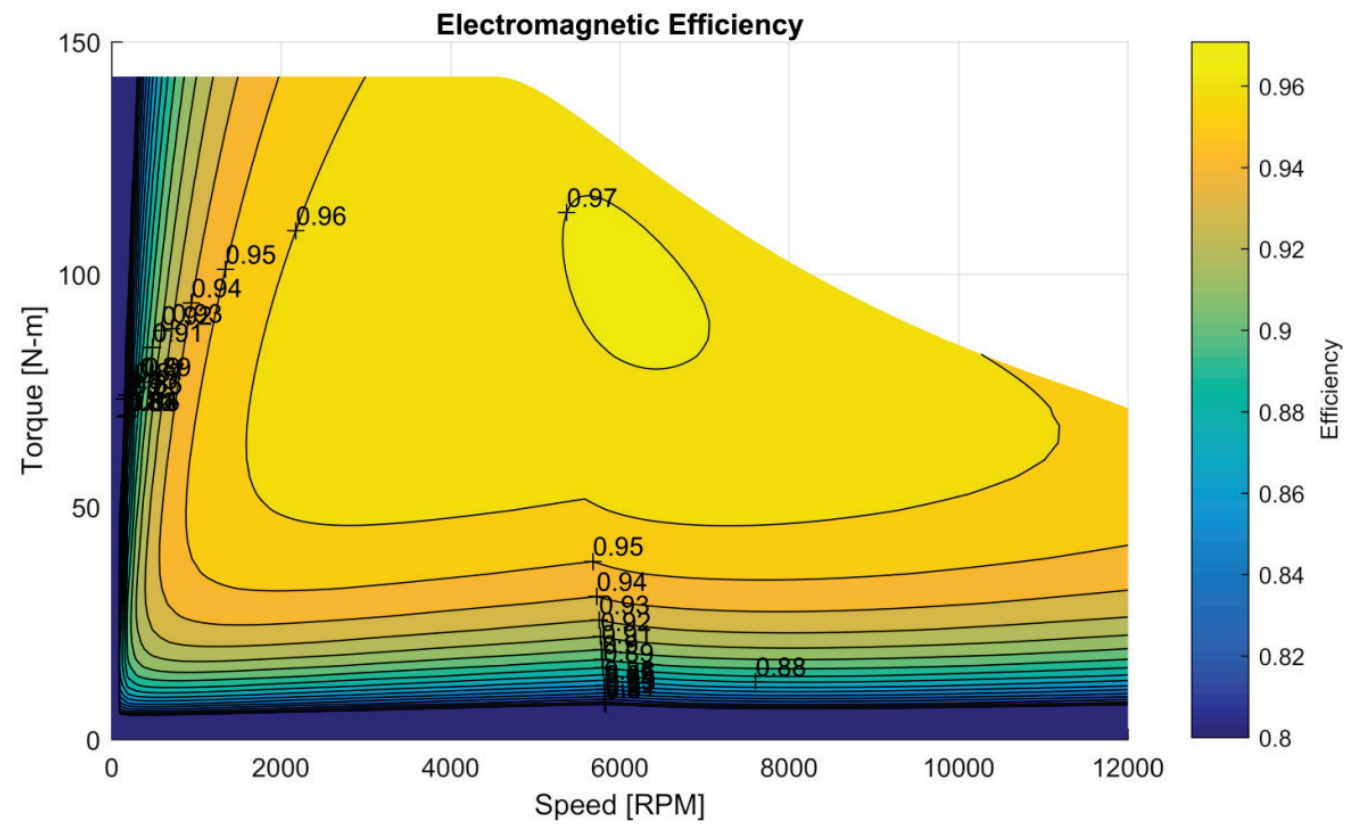

Figure 6: Efficiency map for Design A.

\section{Design B}

A refined analytical model, which includes a more careful consideration of the geometric constraints associated with the rotor topology, yielded a design that maximizes the PM flux linkage. Figure 7 shows an efficiency map for this design, termed Design B. It is qualitatively similar to the one for Design A, as many of the optimal dimensions (e.g., rotor outer radius, normalized tooth dimensions) end up being similar. The main difference is that it includes a slightly thicker magnet, allowing more amp turns and increasing the overall torque and power output of the motor. The demagnetization and core saturation constraints are more closely 
matched in this design. The peak torque is estimated to be $218 \mathrm{~N}-\mathrm{m}$, and the peak power is greater than $110 \mathrm{~kW}$ above $5,000 \mathrm{rpm}$.

An interesting phenomenon observable in the efficiency map is the relatively flat efficiency contours in the field-weakening region (speeds above 5, $000 \mathrm{rpm}$ ) and low torque levels (less than $60 \mathrm{~N}-\mathrm{m}$ ). Because field weakening reduces the amplitude of the flux density in the core, the core losses tend to increase proportionally to speed instead of at the square of the speed, as would be the case without field weakening. Since the power output is also proportional to speed, the efficiency remains relatively constant. Localized heating due to leakage flux may still be a concern.

Figure 7 gives the estimated material volumes and masses for Design B. Compared with the 2010 Prius, the rotor contains a greater proportion of the overall mass at roughly $11.4 \mathrm{~kg}$ total. A disadvantage of this topology is the difficulty of reducing the rotor mass without sacrificing structural integrity. However, the estimated mass is still within a reasonable range compared with other existing systems on the road today.

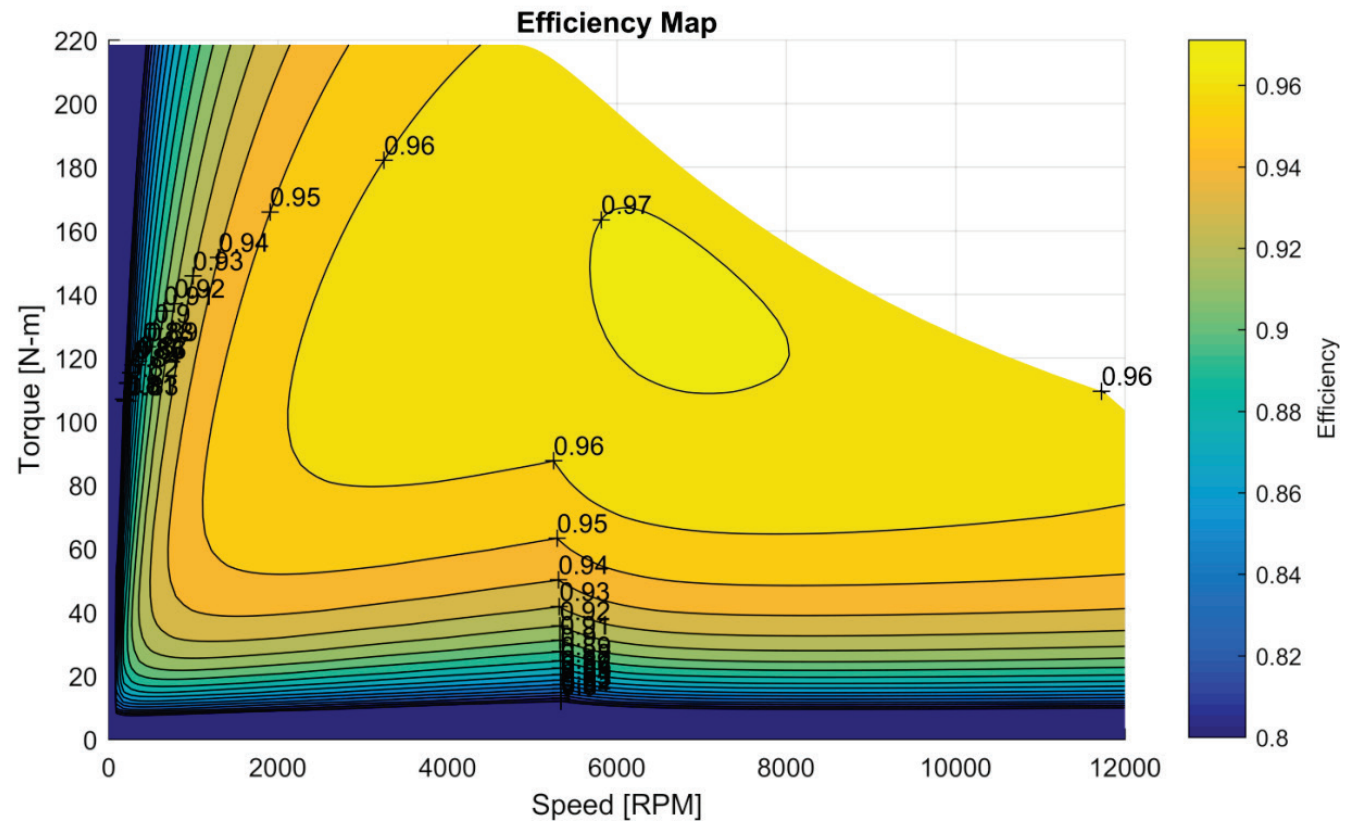

Figure 7: Efficiency map for Design-B.

The total volume of copper is greatly reduced in this design as a result of the concentrated winding scheme. The aspect ratio of the 2010 Prius (stator outer diameter to stack length) is relatively poor at 5:1. This leads to relatively large end turns, with an estimated end-turn-to-active-copper ratio of about 1.5:1. In comparison, the same ratio for the concentrated winding design is closer to $0.5: 1$. This represents a drastic increase in copper volume and losses. Note that Design B has constraints matching those in the 2010 Prius, including a $650 \mathrm{Vdc}$ inverter bus voltage, a 170 Arms current limit, and the same stack length and stator outer diameter. It should be noted that for a comparable volume, the stack length of the concentrated winding design can be increased considerably, since end turns consume much less of the axial volume associated with the motor. A comparison of performance is provided later in this report after other designs are discussed.

Table 1: Estimated material volumes and masses for Design B

\begin{tabular}{|l|c|c|l|}
\multicolumn{1}{|c|}{ Material } & Volume $(\mathrm{L})$ & Mass $(\mathrm{kg})$ & \multicolumn{1}{|c|}{ Note } \\
\hline Rotor laminations & 0.81 & 6.3 & JFE 35H440 \\
\hline Ferrite magnets & 0.67 & 3.5 & Hitachi NMF-12G+ \\
\hline Rotor shaft & 0.21 & 1.6 & Estimate based on 2010 Prius \\
\hline Stator laminations & 0.91 & 7.1 & JFE 35H440 \\
\hline Copper & 0.20 & 1.8 & Assuming a 50\% fill factor \\
\hline
\end{tabular}




\section{Design C}

Refinements were made to the winding layout of Design B to achieve a very low $(\sim 1.7 \%)$ torque ripple with this topology without skewing of the stator or rotor. An example torque waveform is shown in Figure 8 . Some winding approaches to reduce torque ripple tend to excite lower-frequency vibrational modes due to a slower spatial variation of the radial forces between the stator and the rotor. This can be undesirable from a noise perspective, depending on where the vibration frequency falls within the spectrum of human perception. The problem is confounded by the fact that mechanical systems tend to be more efficient at damping highfrequency vibrations.

Additional mechanical simulations are required to evaluate the feasibility of achieving reduced torque ripple in this manner. If possible, it is certainly cheaper from a manufacturing perspective than the method of skewing the stator and/or rotor. The optimal solution involves a compromise that will still reduce the torque ripple, although to a lesser extent, but will suffer fewer negative effects from radial forces.

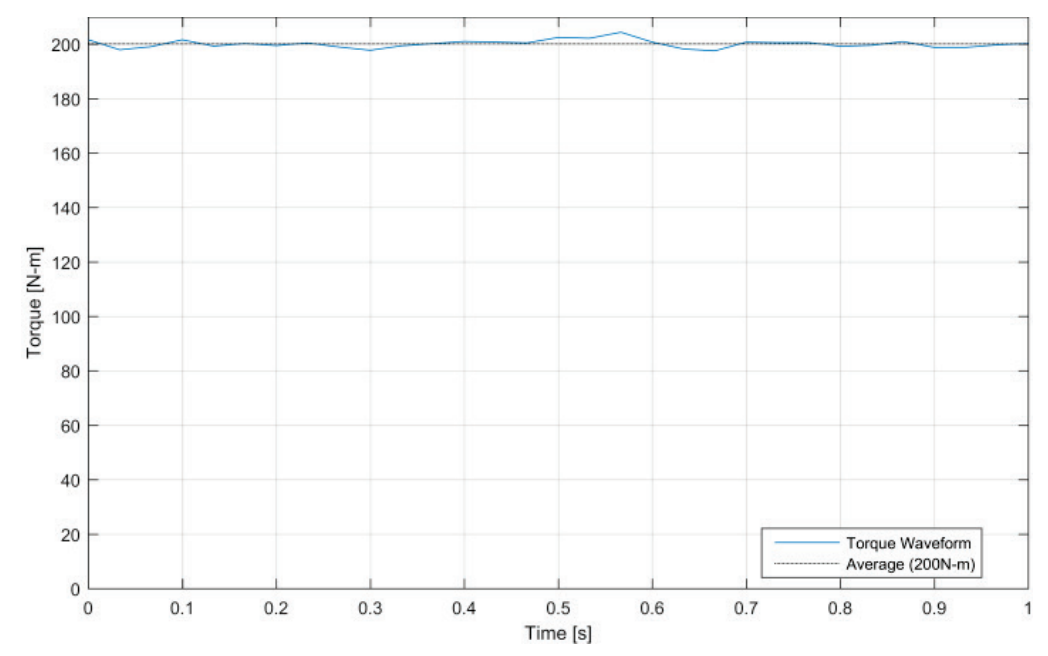

Figure 8: Torque waveform from a design with winding designed for torque ripple minimization.

\section{System Level Considerations-Design D}

The base speed of Design B is about twice that of the 2010 Prius. The fact that the peak torque can be delivered at much higher speeds is directly responsible for the higher output power of the design. This may in fact be unnecessary, and the extra headroom could be used to improve the overall system elsewhere. For example, the current rating of the inverter could be lowered (say, by 50\%) and the number of turns increased in the motor to give similar peak torque and power capabilities to those of the 2010 Prius with a greatly downsized inverter. Or the operating voltage can be lowered so that the electric drive system operates at lower voltages, near those of typical nominal battery voltages in the range of 300-400Vdc. Otherwise, a boost converter may be required.

Figure 9 shows an electromagnetic efficiency map for Design $\mathrm{D}$, a redesigned version of Design $\mathrm{B}$, with twice the number of turns and the peak current rating decreased by half ( $\sim 85 \mathrm{Arms})$. The peak torque remains constant, while the base speed is reduced to $2400 \mathrm{rpm}$. The motor is capable of producing peak power greater than $60 \mathrm{~kW}$ at speeds greater than 2,800 rpm.

The maximum torque versus speed for Design B and Design D is shown in Figure 10, which shows a direct comparison with the values for the 2010 Toyota Prius. Similarly, a plot of power versus speed for Design B, Design D, and the 2010 Prius is provided in Figure 11. Note again that the electrical operating constraints and key geometric parameters (stator outer diameter and stack length) of Design B match those of the 2010 Prius, and a significant improvement of torque and power capability is observed. Note that the 2010 Prius performance curves have been empirically validated, whereas the curves for the various designs discussed previously are from first-round FEA simulations, and slightly lower empirical performance is possible. However, the maximum amount of degradation is expected to be about $10-20 \%$ of the performance represented in the plots. 


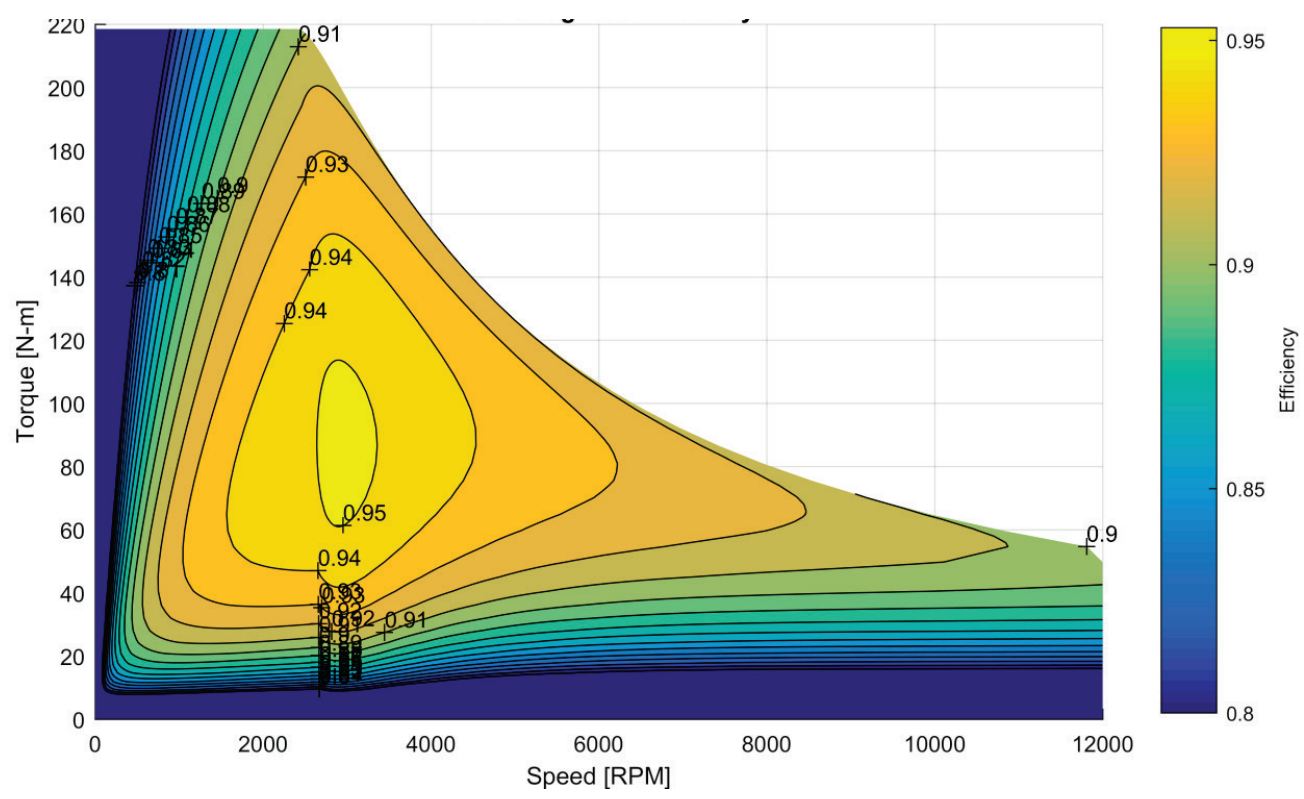

Figure 9: Electromagnetic efficiency contour plot for Design D.

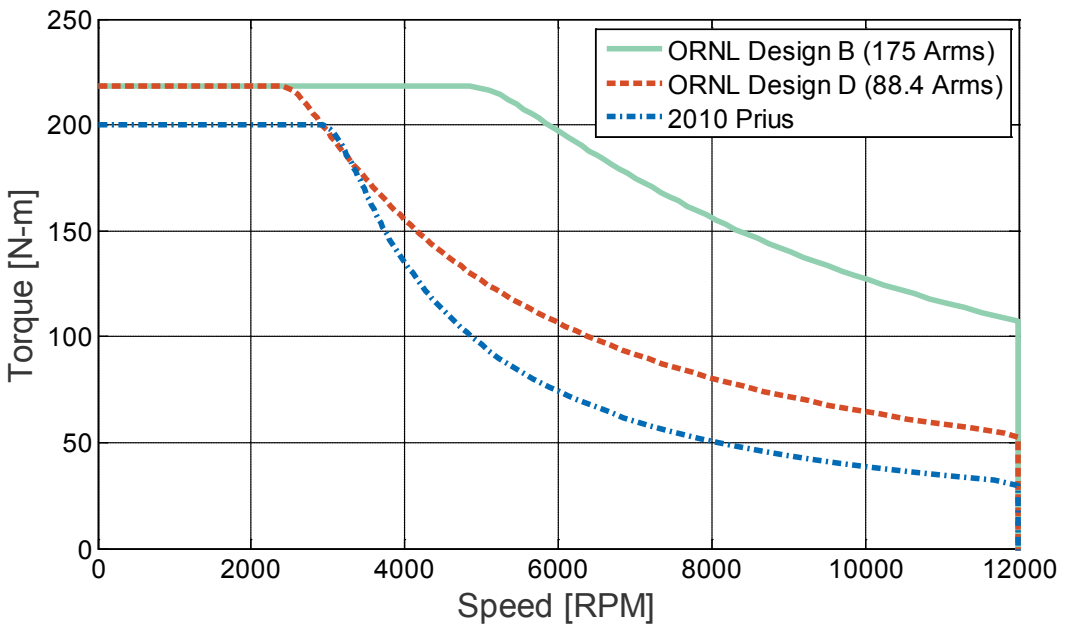

Figure 10: Torque versus speed for ORNL's Design B and Design D compared with that of the 2010 Prius.

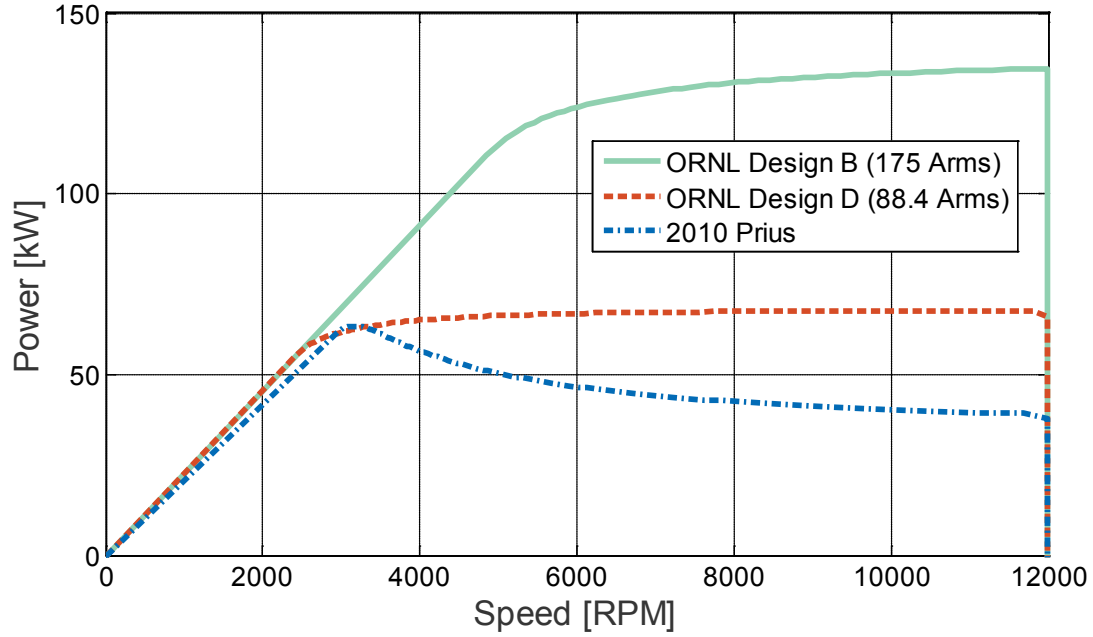

Figure 11: Power versus speed for ORNL's Design B and Design D compared with that of the 2010 Prius. 


\section{Conclusions and Future Direction}

ORNL continues to make advances in the development of low-cost processing methods for the production of high-efficiency Si steel for motor laminations. In this FY 2015 effort, jointly funded by the Propulsion Materials Program, thermomechanical processing of Fe-Si-B steels with different Si and boron contents, sufficient ductility during warm rolling, and significant thickness reductions was achieved without incidence of cracking. Controlled warm deformation studies are under way to optimize the warm rolling conditions that would maximize the subsequent room-temperature ductility for cold rolling of the sheet to the finish thickness.

Simulations are being implemented on ORNL's Titan supercomputer using a Monte Carlo technique to study the fundamental impacts of residual stress, temperature, and other influences upon the magnetic properties of electrical steel that is used in electric motors. Ultimately, this information will be used to help in the development of high-fidelity FEA models.

Motor performance and efficiency metrics from FY 2015 motor design efforts involving the use of ferrite PMs are highly competitive with those for state-of-the-art rare earth PM motors. Table 2 shows a summary of the various targets compared with ORNL's designs and the 2010 Prius design. While the designs still need to be verified with empirical testing, the results are highly encouraging. Secondary prototypes will be fabricated and tested in FY 2016.

Table 2: Comparison of status with DOE targets

\begin{tabular}{|l|c|c|c|c|}
\hline \multicolumn{1}{|c|}{ Parameter } & DOE 2020 targets & ORNL Design B & ORNL Design D & 2010 Prius \\
\hline $\mathrm{kW} / \mathrm{L}$ & 5.7 & 8.8 & 5.1 & 4.8 \\
\hline $\mathrm{kW} / \mathrm{kg}$ & 1.6 & 3.2 & 1.8 & 1.6 \\
\hline Estimated $\$ \mathrm{~kW}$ & 4.7 & 3.1 & 2.2 & 13.5 \\
\hline
\end{tabular}

\section{FY 2015 Presentations/Publications/Patents}

1. T. Burress, et al., "Non-rare earth motor development," presented at the DOE Vehicle Technologies Program Electric Drive Technologies FY 2015 Kickoff Meeting, Oak Ridge, Tennessee, November $18,2014$.

2. T. Burress, et al., "Non-rare earth motor development," presented at the DOE Vehicle Technologies Program Electric Drive Technologies Electrical and Electronics Technical Team Meeting, February 24, 2015.

3. T. Burress, et al., "Non-rare earth motor development," presented at the VTO 2015 Annual Merit Review, Washington DC, June 2015. 


\subsection{Multi-Speed-Range Electric Motors}

Lixin Tang, Principal Investigator

Oak Ridge National Laboratory (ORNL)

National Transportation Research Center

2360 Cherahala Boulevard

Knoxville, TN 37932

Phone: (865) 946-1526

E-mail: tangl@ornl.gov

Susan A. Rogers, DOE EDT Program Manager

Phone: (202) 586-8997

E-mail: Susan.Rogers@ee.doe.gov

Burak Ozpineci, ORNL EDT Program Manager

Phone: (865) 946-1329

E-mail: burak@ornl.gov

Contractor: UT-Battelle, LLC, managing and operating contractor for the Oak Ridge National Laboratory Contract No.: DE-AC05-00OR22725

\section{Abstract/Executive Summary}

- In this project, multi-speed-range (MSR) solutions for electric motors are being developed to improve drive cycle efficiency, increase power density/specific power, and reduce system cost. In FY 2015, we used drive cycle simulation to confirm the effectiveness of MSR operation, developed a new design to achieve MSR operation, and finished initial benchtop tests.

\section{Accomplishments}

- Confirmed by drive cycle simulation that MSR operation achieves higher drive cycle efficiency.

- Used a 3-speed-range motor to reduce the total loss by $24 \%$ in a combined drive cycle.

- Developed a novel MSR system design.

- It features fewer added solid-state ac switches and lower losses in low-speed and high-speed mode.

- It does not have a de short-circuit failure mode.

- Conducted a benchtop component test.

- Designed/fabricated a low-cost fast switching ac switch printed circuit board (PCB) assembly for changing switching arrangements.

- Confirmed the capability to decrease current, change winding configurations, and increase current in a short amount of time.

\section{Introduction}

The latest benchmarking test results for different hybrid electric vehicle (HEV) and plug-in electric vehicle (PEV) drive systems show that single-speed-range drive systems normally are most efficient in a high-power region-i.e., medium torque in a medium- to high-speed area- and less efficient in low torque and speed regions. To achieve high efficiency, it is desirable to operate the drive system as close as possible to the highefficiency area. Figure 1 is an efficiency map of an EV drive system at different torques and speeds. Its efficiency ranges from $70 \%$ at around $500 \mathrm{rev} / \mathrm{min}$ to a peak efficiency of $96 \%$ at around $6,400 \sim 8,600 \mathrm{rev} / \mathrm{min}$ with $80 \sim 100 \mathrm{Nm}$ load torque. 


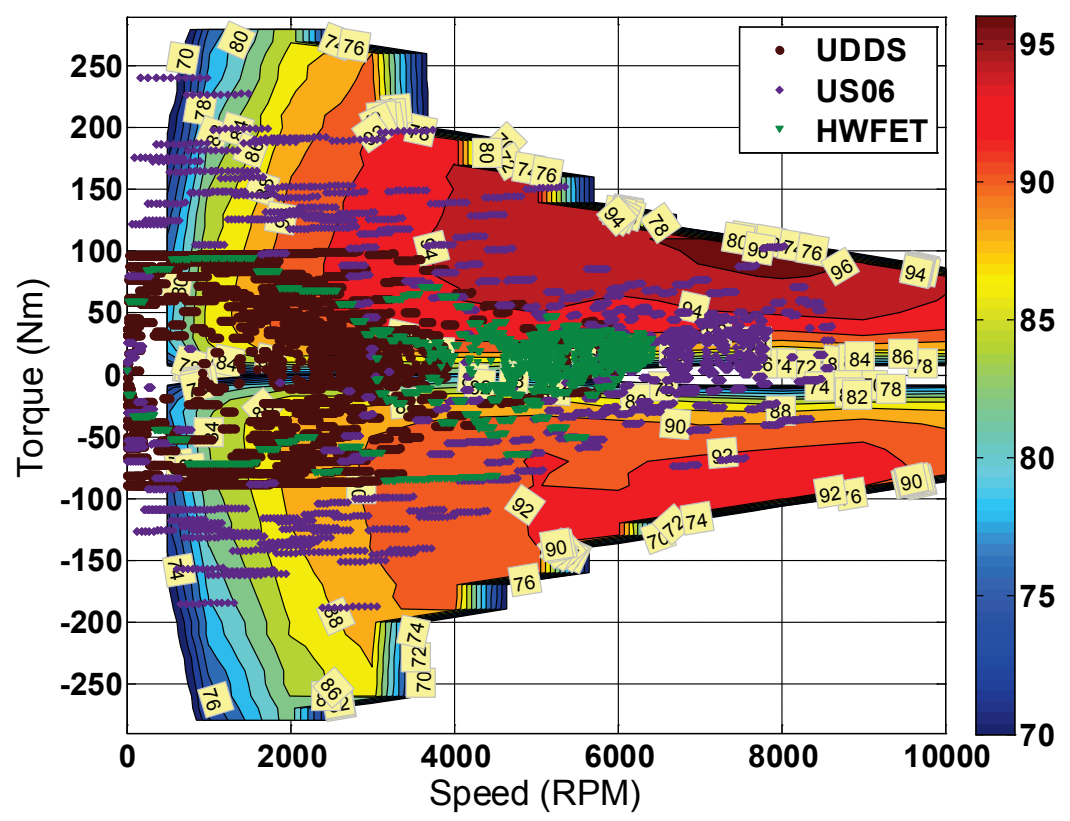

Figure 1: Overlay of drive cycle operation points on efficiency map.

In actual PEV operation, the motor operating point is determined by multiple factors, such as dc bus voltage, vehicle speed, vehicle weight, road condition, and wind speed and direction. Normally, most drive conditions can be represented by drive cycles such as the US06 Supplemental Federal Test Procedure (US06) (simulates aggressive driving), Urban Dynamometer Driving Schedule (UDDS) (simulates city stop-and-go traffic), and the Highway Fuel Economy Test (HWFET ) (simulates highway traffic).

If the different driving cycle operation points are superimposed onto the drive system torque-versus-speed efficiency map, the system efficiency for each operating point can easily be seen. It is noticeable that most operating points of the UDDS (brown points) are limited to $0 \sim 4,000 \mathrm{rpm}$ and to the $-100 \sim 100 \mathrm{Nm}$ region. The HWFET (blue) points are mostly $0 \sim 50 \mathrm{Nm}$ and 5,000 6,000 rev/min; neither is in the peak efficiency area. The US06 (purple) points are spread over a larger area $(0 \sim 9,000 \mathrm{rev} / \mathrm{min}$ and $-200 \sim 250 \mathrm{Nm})$ on the image; however, there are very few points in the peak efficiency area. As a result, it can be seen that the peak efficiency region is very infrequently used in most drive cycles and driving conditions.

In this project, we are developing MSR solutions to improve drive cycle efficiency by shifting the peak efficiency region to the region in which the motor most frequently operates. Reducing drive cycle power losses will increase the system power density/specific power and reduce cost. Also under consideration are the important impacts and advantages of changing torque-speed characteristics compared with mechanical hydraulic transmission systems.

\section{Approach}

The project objective is to improve drive cycle efficiency by facilitating drive system operation in highefficiency regions. With reduced system total losses, the power density/specific power should be improved and the cost should be reduced. Literature reviews were and will be conducted throughout the project to make sure the approach is unique. After the initial literature review was conducted, the following approaches were considered to achieve MSR operation.

- Electric motor designs

- Winding arrangements (e.g., reconfigurable windings)

- Power electronics integration

- Control techniques 
Figure 2 shows an example of MSR operation in the UDDS drive cycle, which indicates stop-and-go driving characteristics typically experienced in cities. The speed range transition levels are set at 15 and $27 \mathrm{mph}$ with three different operating modes that are determined by the vehicle speed. A transition occurs if the vehicle speed crosses one of the two speed transition levels, and it is desirable that each transition be smooth, short, and stable.

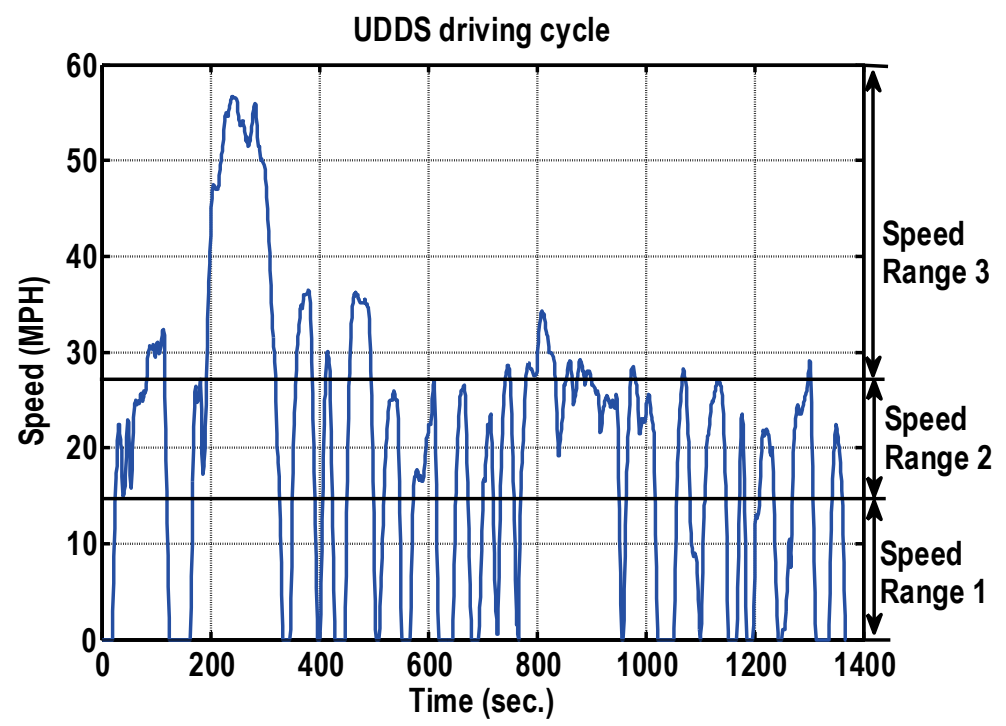

Figure 2: Example of 3-speed-range operation in UDDS drive cycle.

This approach can be viewed as mimicking the functionality of a mechanical transmission. A 3-speed transmission designed for PEV applications is shown in Figure 3 to show the complexity, weight, and volume of a mechanical transmission. It weights about $45 \mathrm{~kg}$, and its overall length can be as much as $16.3 \mathrm{in}$. The oil pumps are heavy and occupy a large volume. A wet clutch requires maintenance and is the component most likely to fail in a conventional transmission. Figure 4 shows a 6-speed transmission for a 2011 Hyundai Sonata hybrid vehicle. A large oil pump and valve body are visible on the left, and some of the clutches and gears of the transmission are shown on the right. Normally, hydraulic clutches are mechanically complicated. They need oil pumps to maintain the pressure - requiring extra power and control — and extra sensors, valves, and a control unit. Our approach will replace a significant number of these parts in a mechanical transmission. Note that an electric drive system may not need a 6-speed transmission because it can generate peak torque at zero speed.

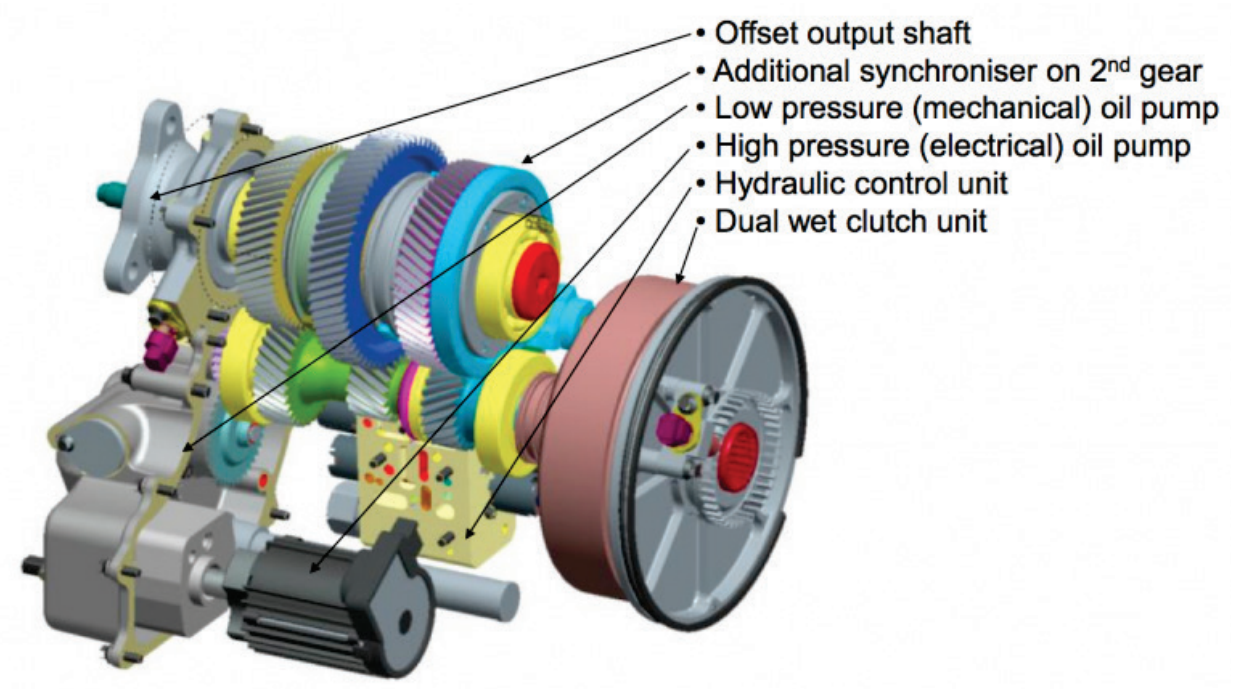

Figure 3: A commercial 3-speed transmission for PEV applications. 

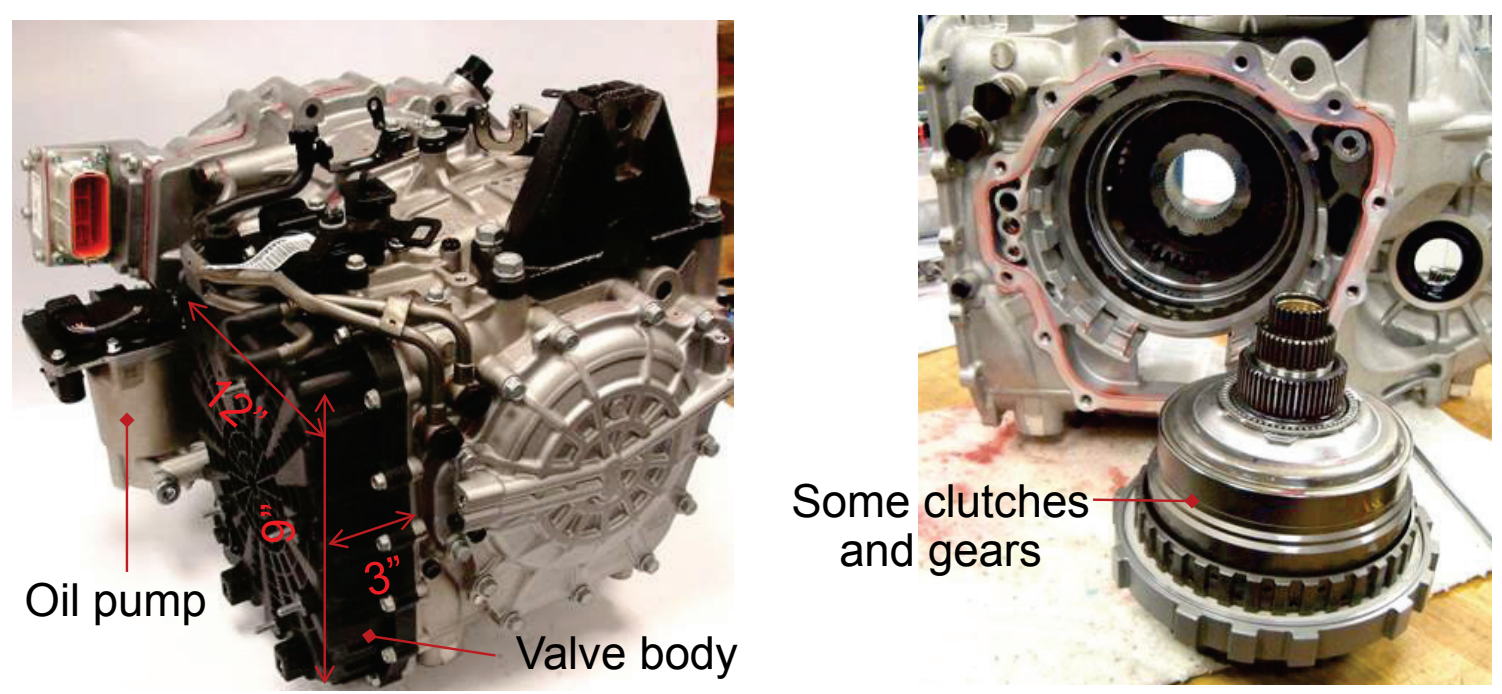

Figure 4: 2011 Hyundai Sonata hybrid 6-speed transmission and its key parts.

\section{Results and Discussion}

\section{Literature review}

MSR operation of electric motors is not a new idea. Some products based on MSR motors are already on the market. For example, some 3-phase induction motors use a star-delta soft-starter to reduce the inrush current when they start. Such a motor initially selects a star winding, which has a larger impedance and backelectromotive force (emf); then when the motor builds up its speed and back-emf, it switches to a delta winding arrangement so that it can go to higher-speed /power operation. This is a 2-speed-range system based on a switchable winding approach. Recently, more papers/patents report progress on MSR motors for automotive applications. Generally, four different approaches are used. The first, a dual-rotor motor approach by Longya Xu, Dorrel, and Boldea, is still in the laboratory prototype stage. It uses two rotors in the drive to achieve a wider speed range and higher efficiency. Often, more than one inverter is needed to drive a dual-rotor motor. These motors are normally mechanically and electrically complicated. A second approach - the use of a mechanical gearbox (transmission) - was not considered in this project because it is not related to electric drive technologies. A third approach, switchable winding, is the one on which most researchers are working. For example, Eckart and Erik worked on switchable winding for a surface-mounted permanent magnet synchronous motor and published several papers around 1999. They proposed and verified a scheme to mitigate torque transient. Chen and Cheng, by using relays, developed a motor with an MSR winding for electrical transmission applications and with brushless dc motors to expand the speed range. Huang and Chang developed switchable winding in an induction motor to achieve faster motor start-up. Fulton, Bates, Krieger, and Shum filed different patents recently for switchable winding motors. This approach uses ac switches to change the winding configuration so that the motor operating mode can be changed to achieve a wider speed range and faster motor starting. Generally, this approach adds complexity to the system. A fourth approach uses pole-change motors to achieve MSR operation. Most of these methods are suitable only for induction motors.

Figure 5 illustrates two important industry patents on this topic. ${ }^{1,2}$ At left is a diagram representing patent A, granted in 2012 for a system in which eight ac switches are used to change the star-connected stator winding between parallel and serial connections. In parallel mode, the five blue ac switches are on and the three orange switches are off; in serial mode, the five blue switches are off and the three orange switches are on. The diagram at right in Figure 5 (patent B) represents a system patented in 2012 for electric bike applications. It uses only three ac switches rather than eight. In parallel mode, $K_{P 1}$ and $K_{P 2}$ are on and $K_{S}$ is off; in serial mode, $K_{S}$ is on and $K_{P 1}$ and $K_{P 2}$ are off. However, this system must use two small inverters instead of one. Further, note that all three switches must be installed in a dc bus; that arrangement may add up the total dc bus stray inductance, which will in turn increase the voltage spikes on the dc bus. This makes it less suitable for high-power applications. Note also that patent B also has a dc short-circuit failure mode if all three switches are on at the same time, and some extra protection should be considered. 

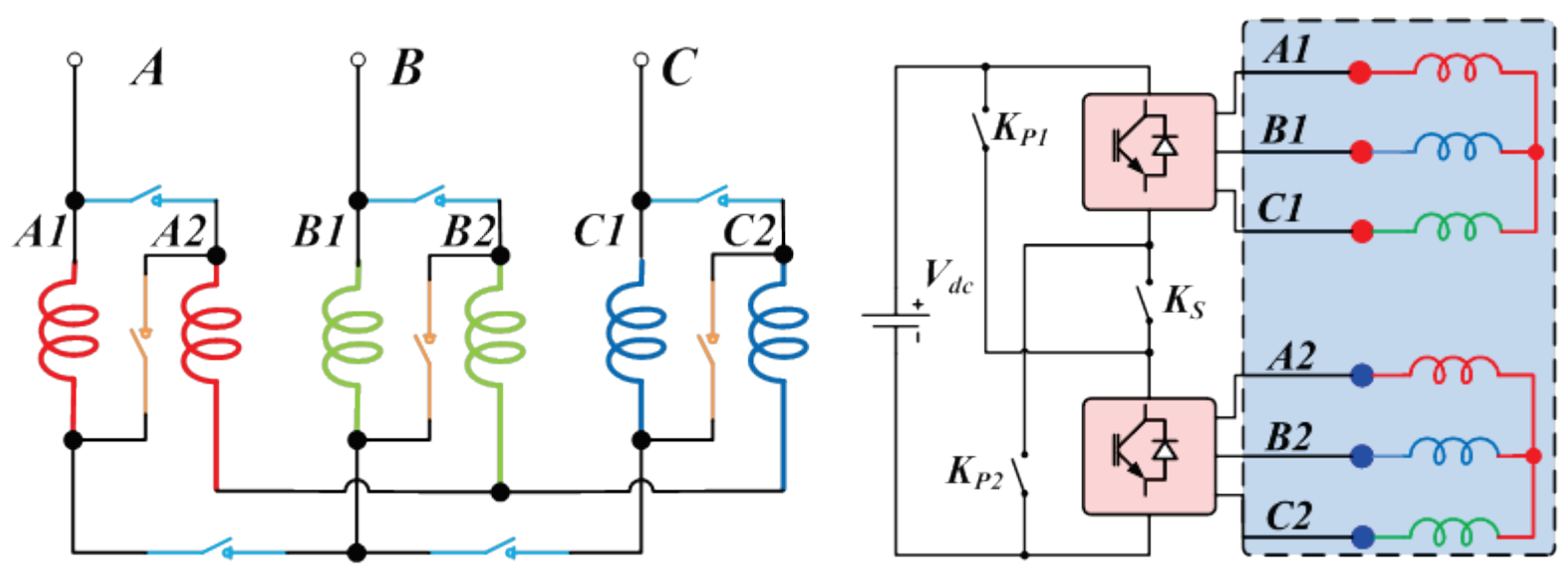

Figure 5: Schematics illustrating two industry patents: (left) patent A-US8415910 B2 (published in 2013 by Fulton) and (right) patent B-US20120086380 (published in 2012 by Krieger and Shum).

\section{Drive cycle simulation}

The peak efficiency region of an electric drive system is normally not the region in which the motor most frequently operates. To study the potential to improve drive cycle efficiency, drive cycle simulations were conducted. Two ratios were used to shift the efficiency map. Figure 6 compares the original efficiency map of an electric drive system with a 2:1 reduction, a 3:1 reduction, and an optimal map in which the condition is chosen based on the highest efficiency. The maps show that when a reduction is used, the peak efficiency map is compressed to the left and stretched upward. In the optimal map, there are two areas with a peak efficiency of 95\%: one at 2,000 3,000 rev/min, 100 170 Nm and the other at 5,000 7,000 rev/min and 50 90 Nm. The 93 and $91 \%$ efficiency contours also expand to the low-speed area.
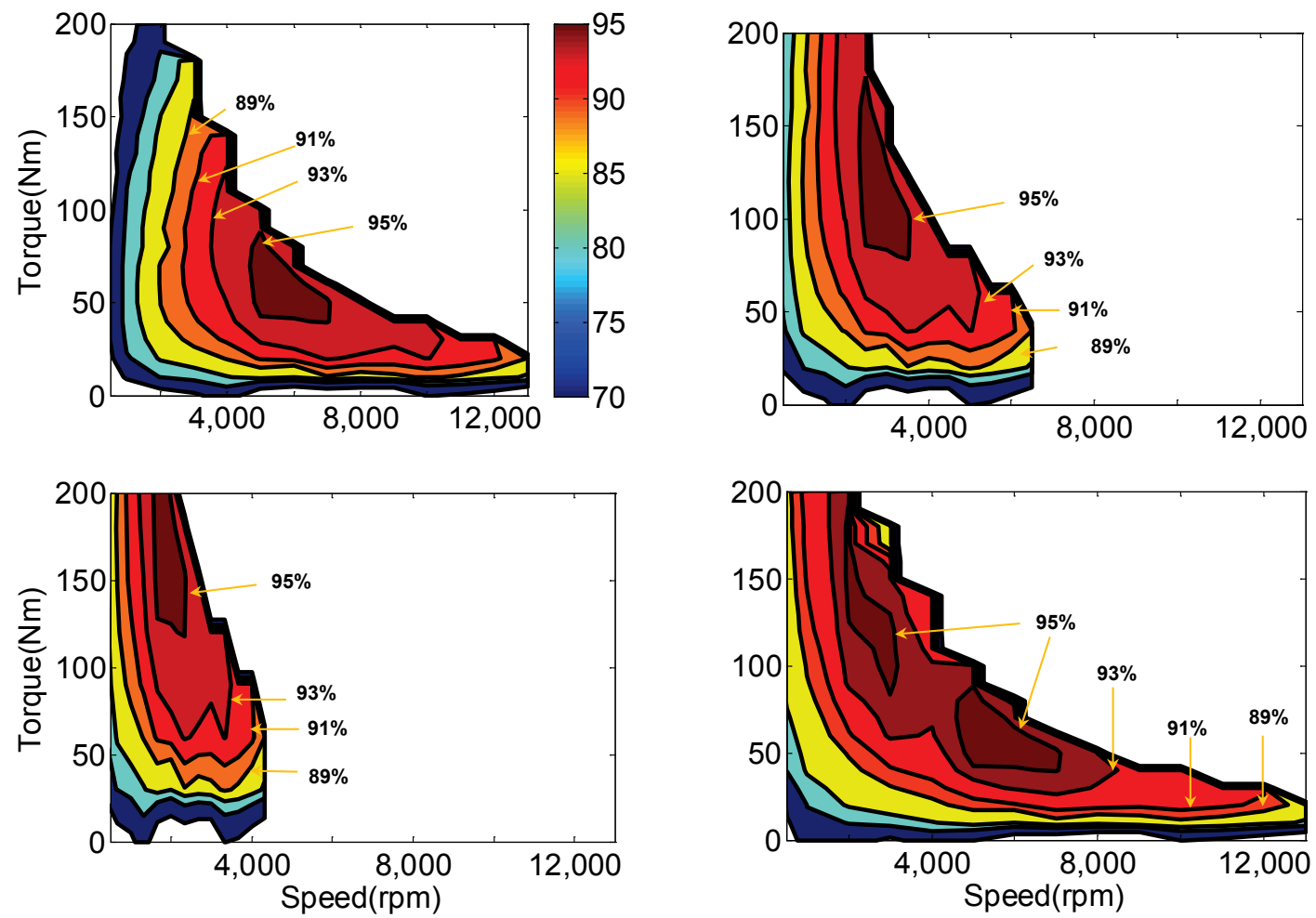

Figure 6: Original Prius drive system efficiency map (top left), system efficiency with 2:1 reduction (top right), system efficiency with 3:1 reduction (bottom left), and optimized system efficiency map (bottom right). 
To study drive cycle efficiency, three scenarios were considered. The first scenario was the original Prius drive system with a fixed-speed-range motor; the second and third scenarios used the Prius system with 2-speed and 3 -speed ratios. Four drive cycles (US06, UDDS, HWFET, and a combined cycle including all three) were selected to compare the drive cycle efficiency.

Table 1 shows the average drive cycle efficiency. The option-1 system is a 2-speed-range drive, and the option-2 system is a 3-speed-range drive. It can be seen that in the MSR system, the drive cycle efficiency is improved most significantly in the UDDS cycle, followed by the US06 cycle and the HWFET cycle. The improvement in the HWFET cycle is low because the operating points were already in a relatively highefficiency area. The option-2 system is more efficient than the option-1 system with only two speed ranges.

Table 1: Average drive cycle efficiency

\begin{tabular}{|l|l|l|l|l|}
\hline & US06 (aggressive ) & $\begin{array}{c}\text { UDDS (city/stop- } \\
\text { and-go) }\end{array}$ & $\begin{array}{c}\text { HWFET } \\
\text { (highway) }\end{array}$ & \multicolumn{1}{|c|}{ Combined } \\
\hline $\begin{array}{l}\text { 2013 Prius motor } \\
\text { only }\end{array}$ & 86.9 & 80.7 & 89.9 & 84.7 \\
\hline $\begin{array}{l}\text { 2013 Prius inverter } \\
\text { only }\end{array}$ & 91.8 & 81.0 & 95.95 & 87.6 \\
\hline 2013 Prius system & 81.7 & 69.1 & 86.6 & 76.7 \\
\hline Option-1 system & 83.6 & 75.5 & 87.5 & 81.5 \\
\hline Option-2 system & 86.3 & 78.7 & 87.8 & 83.0 \\
\hline
\end{tabular}

Table 2 shows the total energy loss of the original and option- 2 system. The results show that with the 3 -speedrange system, the total energy loss can be reduced by 29.7, 28, 4.6, and 23.6\% in the US06, UDDS, HWFET, and combined cycles, respectively. All the results show that an MSR system can improve drive cycle efficiency and reduce drive cycle loss significantly.

Table 2: Drive cycle total energy loss

\begin{tabular}{|l|l|l|l|l|}
\hline & \multicolumn{1}{|c|}{$\begin{array}{c}\text { US06 } \\
(\times 1,000,000 \\
\text { Joules })\end{array}$} & $\begin{array}{c}\text { UDDS } \\
(\times 1,000,000 \\
\text { Joules })\end{array}$ & $\begin{array}{c}\text { HWFET } \\
(\times 1,000,000 \\
\text { Joules })\end{array}$ & $\begin{array}{c}\text { Combined } \\
(\times 1,000,000 \\
\text { Joules })\end{array}$ \\
\hline 2013 Prius system & 1.374 & 1.621 & 0.803 & 3.798 \\
\hline Option-2 system & 0.966 & 1.167 & 0.766 & 2.899 \\
\hline Loss reduction (\%) & 29.7 & 28.0 & 4.61 & 23.6 \\
\hline
\end{tabular}

\section{New MSR designs}

MSR designs based on a switchable winding approach were proposed to achieve MSR operation; the block diagram can be seen in Figure 7. Compared with the patent A approach shown in Figure 5, the proposed design uses fewer ac power switches; and because it does not insert ac power switches in the dc bus, it does not have the dc short-circuit failure mode that patent $\mathrm{B}$ has. To evaluate the advantages, the inverter losses of the design proposed in patent A were compared with those of the MSR design. The inverter loss includes switching loss and conduction loss. The basic equations used to calculate switching loss and conduction loss in a 3-phase voltage source inverter can be seen in Eqs. (1-3). Equation (1) shows the inverter switching loss, where $f_{S W}$ is the switching frequency, $V_{d c}$ is the dc bus voltage, $I_{P E A K}$ is the peak phase current, $V_{r e f}$ and $I_{r e f}$ are the 
voltage and current at which the switching losses are given, $E_{O N_{-} I G B T}, E_{O F F_{-} I G B T}$ are the turn-on, turn-off loss of the insulated gate bipolar transistor (IGBT), and $E_{O F_{-} D I O D E}$ is the turn-off loss of the diode.

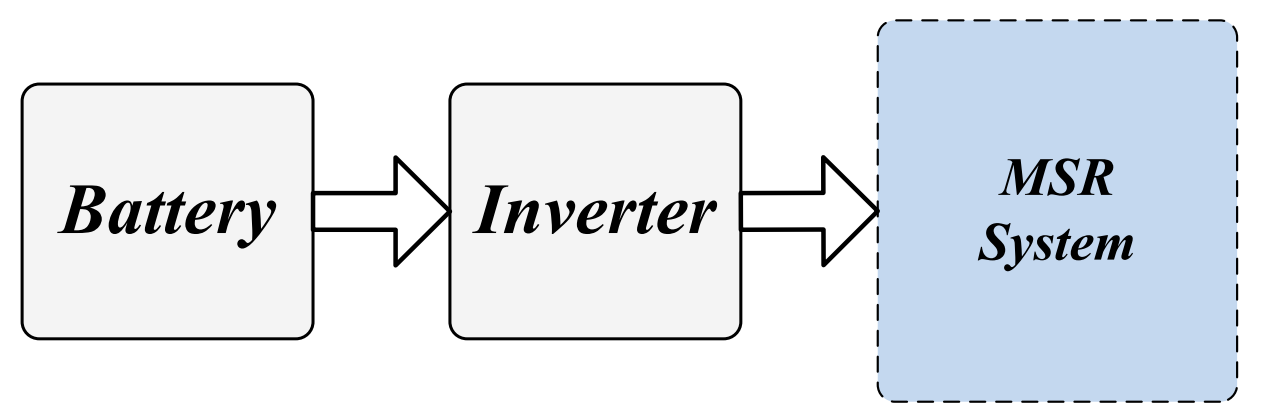

Figure 7: Proposed MSR design.

Equation (2) shows the conduction losses of the IGBT, in which $V_{C E O}$ and $r_{I G B T}$ are the zero current saturation voltage and equivalent resistance of the IGBT, $m$ is the modulation index, and $\theta$ is the power displacement factor. Equation (3) shows the conduction losses of the diodes, in which $V_{f o}$ and $r_{d}$ are the zero current forward voltage and equivalent resistance of the diode. With Eqs. (1) to (3), the voltage source inverter total loss can be calculated. The losses associated with the solid-state ac switches for switching winding configurations are also considered in Eq. (4), where $V_{C E R 0}$ is zero current forward voltage drop and $r_{R}$ is the equivalent resistance of the solid-state ac switch (relay). The key parameters of an off-the-shelf IGBT module with a $1200 \mathrm{~V} / 450 \mathrm{~A}$ rating were used in the simulation. A switching frequency of $7.5 \mathrm{kHz}$ was used with a $650 \mathrm{~V}$ dc bus voltage, and the motor peak phase current was $250 \mathrm{~A}$.

$$
\begin{aligned}
& P_{S L}=\frac{6 f_{s w} V_{d c} I_{\text {Peak }}}{\pi V_{\text {ref }} I_{\text {ref }}} \times\left(E_{\text {ON_IGBT }_{-}}+E_{\text {OFF_IGBT }}+E_{\text {OFF_DIODE }}\right) \\
& P_{C L_{-} I G B T}=I_{P E A K} V_{C E O}\left(\frac{1}{2 \pi}+\frac{1}{8} m \cos \theta\right)+I_{P E A K}^{2} r_{I G B T}\left(\frac{1}{8}+\frac{1}{3 \pi} m \cos \theta\right) \\
& P_{C L_{-} D}=I_{P E A K} V_{f 0}\left(\frac{1}{2 \pi}-\frac{1}{8} m \cos \theta\right)+I_{P E A K}^{2} r_{d}\left(\frac{1}{8}-\frac{1}{3 \pi} m \cos \theta\right) \\
& P_{C L_{-} R}=I_{P E A K} V_{C E R 0}\left(\frac{2}{\pi}+\frac{I_{P E A K}^{2} r_{R}}{2}\right)
\end{aligned}
$$

Figure 8 shows the total inverter losses of different approaches. The trace with red squares shows the inverter loss of a fixed winding motor at different peak phase currents from $50 \mathrm{~A}$ up to $250 \mathrm{~A}$. The trace with blue diamonds shows the loss of the patent A design at high speed; the trace with brown diamonds shows the loss of the proposed MSR design at high speed. The trace with blue triangles shows the loss of the patent A design in low-speed operation; the trace with brown triangles shows the loss of the proposed MSR design at low speed. It can be seen that the patent A design has the highest loss, while the fixed winding motor has the second highest loss. It is clear that the proposed MSR design has lower losses in both low-speed and high-speed operation compared with the fixed winding motor. For high speeds, the loss reduction is $24.6 \%$, and for low speeds, it can be as high as $43.2 \%$. Compared with the patent A approach, the proposed MSR design achieves a significant loss reduction of $33.7 \%$ at high speed. Note that the loss at low speed is slightly higher $(+3.3 \%)$ than that of the patent A approach. Table 3 summarizes the loss reductions of the MSR and patent A approaches at low-speed and high-speed operation with maximum motor current. The results show the proposed MSR design can reduce power loss much more than the approach proposed in patent A. 


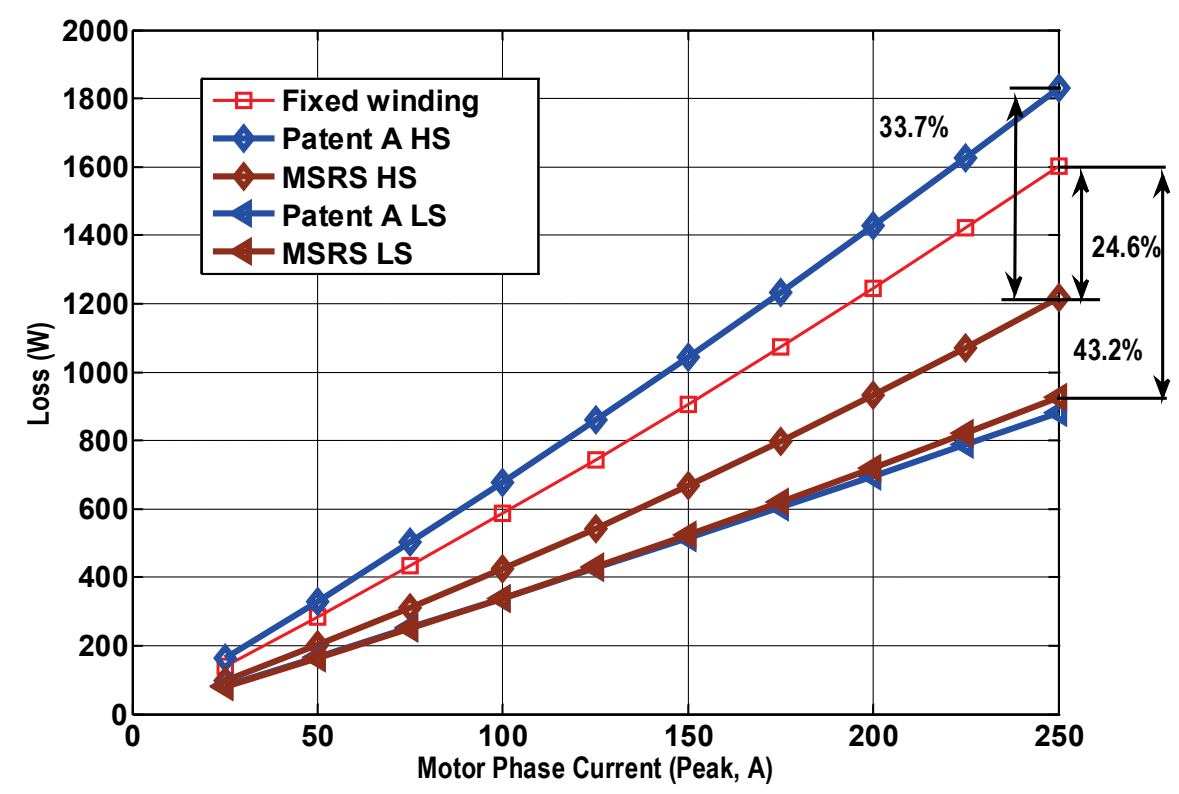

Figure 8: Loss simulation of the proposed MSR, fixed winding drive, and patent A system.

Table 3: Loss comparison

\begin{tabular}{|l|c|c|c|}
\hline & $\begin{array}{c}\text { Fixed winding } \\
\text { motor loss }(\mathrm{W})\end{array}$ & $\begin{array}{c}\text { SOA system } \\
\text { loss }(\mathrm{W})\end{array}$ & $\begin{array}{c}\text { MSR system } \\
\text { loss }(\mathrm{W})\end{array}$ \\
\hline $\begin{array}{l}\text { Maximum current in } \\
\text { LS mode }\end{array}$ & 1620 & 890 & 920 \\
\hline $\begin{array}{l}\text { Maximum current in } \\
\text { HS mode }\end{array}$ & 1620 & 1840 & 1220 \\
\hline $\begin{array}{l}\text { Loss change over fixed } \\
\text { winding motor(LS/HS) }\end{array}$ & $\begin{array}{l}100 \%(\mathrm{LS}) \\
100 \%(\mathrm{HS})\end{array}$ & $-45 \%(\mathrm{LS})$ & $-43.2 \%(\mathrm{LS})$ \\
\hline
\end{tabular}

\section{Switching transient and benchtop component test}

To actually change the winding configuration, the drive will have some switching transient. During the transient, the controller will first need to bring all the phase currents down to zero. Second, the ac switches will turn on/off to change the winding configuration at zero phase current. Finally, the controller will build up the 3 -phase current to the new operating point. Figure 9 shows a switching transient of a 3-phase motor with peak torque. At $198 \mathrm{~ms}$, the inverter starts to turn off the 3-phase current. It takes roughly $3 \mathrm{~ms}$ to completely turn off all 3-phase current; then the ac switches are given $9 \mathrm{~ms}$ to change the winding configuration. The current ramp-up and ramp-down times are determined by the dc bus voltage and motor parameters such as the inductance and flux linkage of the permanent magnet. Since all 3-phase currents are zero, there is a torque interruption to the drive, which is not desirable. This torque interruption time is determined by the speed of the ac switches. If fast solid-state ac switches are used, each turn on/turn off takes only about $1 \sim 2 \mathrm{~ms}$; the torque interruption time could be shorter. However, if slow ac switches like ac contactors are used, the undesirable torque interruption could be longer. Further, the ac contactors have limited operating cycles because of the mechanical parts. All these drawbacks make the ac contactor unattractive for automotive applications. To minimize the torque interruption, fast and low-cost solid-state ac switches were selected in this project. We also looked at other techniques to mitigate the torque interruption. 
I(A)
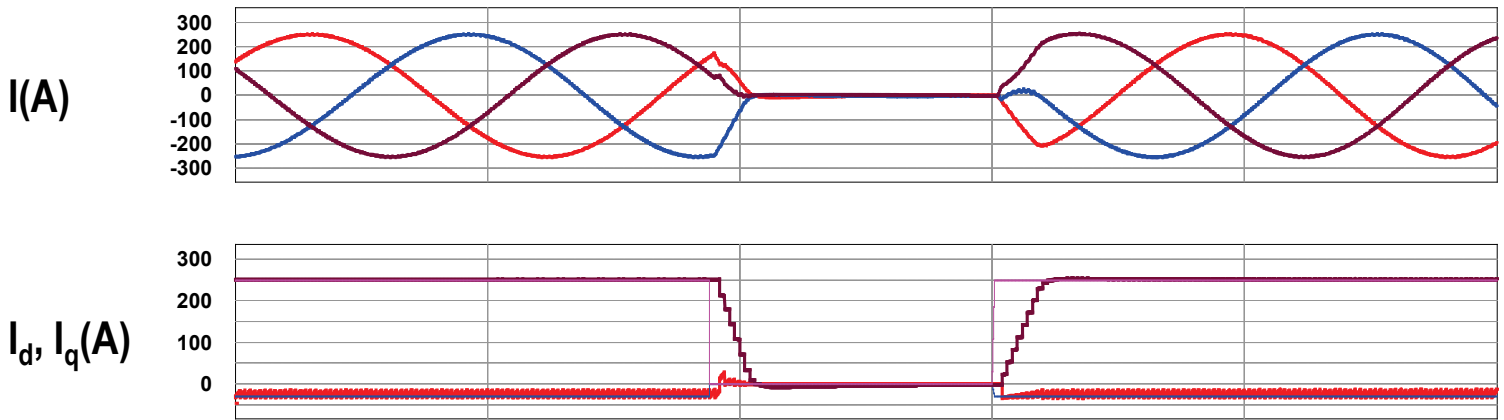

Torque interruption - about $14 \mathrm{~ms}$

$\mathrm{T}(\mathrm{Nm})$

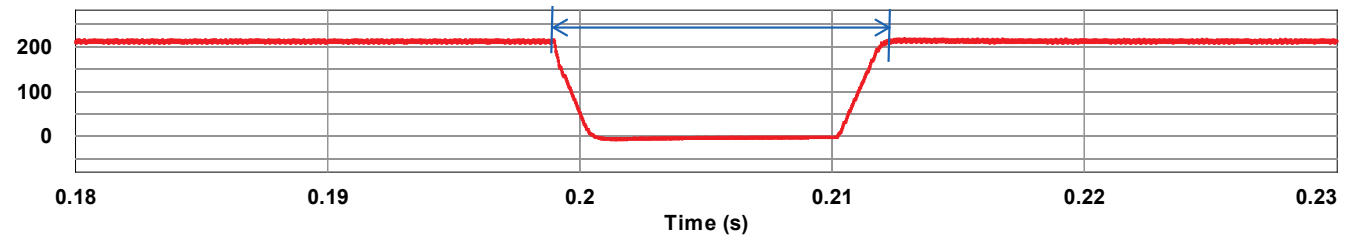

Figure 9: Loss simulation of the proposed MSR, fixed winding drive, and patent A system.

A $4.2 \times 4.3$ in. PCB was designed to switch the motor 3-phase winding. Anti-parallel thyristor modules were used as the ac switches. There are off-the-shelf, solid-state ac switches on the market. However, they are fairly expensive - more than $\$ 100$ for a switch with a $660 \mathrm{~V} / 125 \mathrm{~A}$ ac rms rating. An anti-parallel thyristor module with a similar rating costs only $\$ 15.14$ (for a quantity $>1,680$ ). It also has a smaller footprint than the off-theshelf solid-state ac switches. Although IGBTs and metal-oxide-semiconductor field-effect transistors can be considered for this application, their high cost is a limiting factor. Further, two IGBTs must be connected in anti-series mode to provide reverse-blocking capability, adding up the conduction loss. IGBTs with reverseblocking capability could be used; however, the forward voltage drop of a reverse-blocking IGBT is higher than that of a normal IGBT, and their market availability is limited at this time.

An HEV motor with a 3-phase winding was used in the test. To simulate a switch winding transient, the winding was rearranged. A single-phase inverter controlled by a digital signal processor was used for the realtime test. Figure 10 shows the ac switch PCB assembly and the motor winding. At top left is the top view of the PCB and at bottom left is the bottom view. Three solid-state ac switches were installed. At right is the motor, which was used in a commercial HEV drive system. Figure 11 shows the test diagram. The winding configuration can be changed between parallel and serial connection by turning the three ac switches on and off.

Figure 12 shows the test waveform. The waveform shows the inverter output current in a transient from series mode (A-B-C-D) to parallel (E-F-G). The ac switches change the configuration of the windings between points $\mathrm{D}$ and $\mathrm{E}$. The inverter current ramp-up/ramp-down time during the transient is about $2.45 \mathrm{~ms}(1.75 \mathrm{~ms}$ from C to $\mathrm{D}$ and $0.7 \mathrm{~ms}$ from $\mathrm{E}$ to F), and the total transient is only $5.0 \mathrm{~ms}$. Since the serial winding configuration has higher inductance, it takes a longer time to ramp up/ramp down the current than it does in parallel mode. A dc bus voltage of $100 \mathrm{~V}$ was used in the test. Note that the current ramp-up/ramp-down time will be much faster with typical hybrid bus voltages in the range of 300-700 Vdc. The test results show the designed ac switch PCB assembly can change the motor winding configuration in a fairly short time. Research continues to be done to further reduce the transient. 


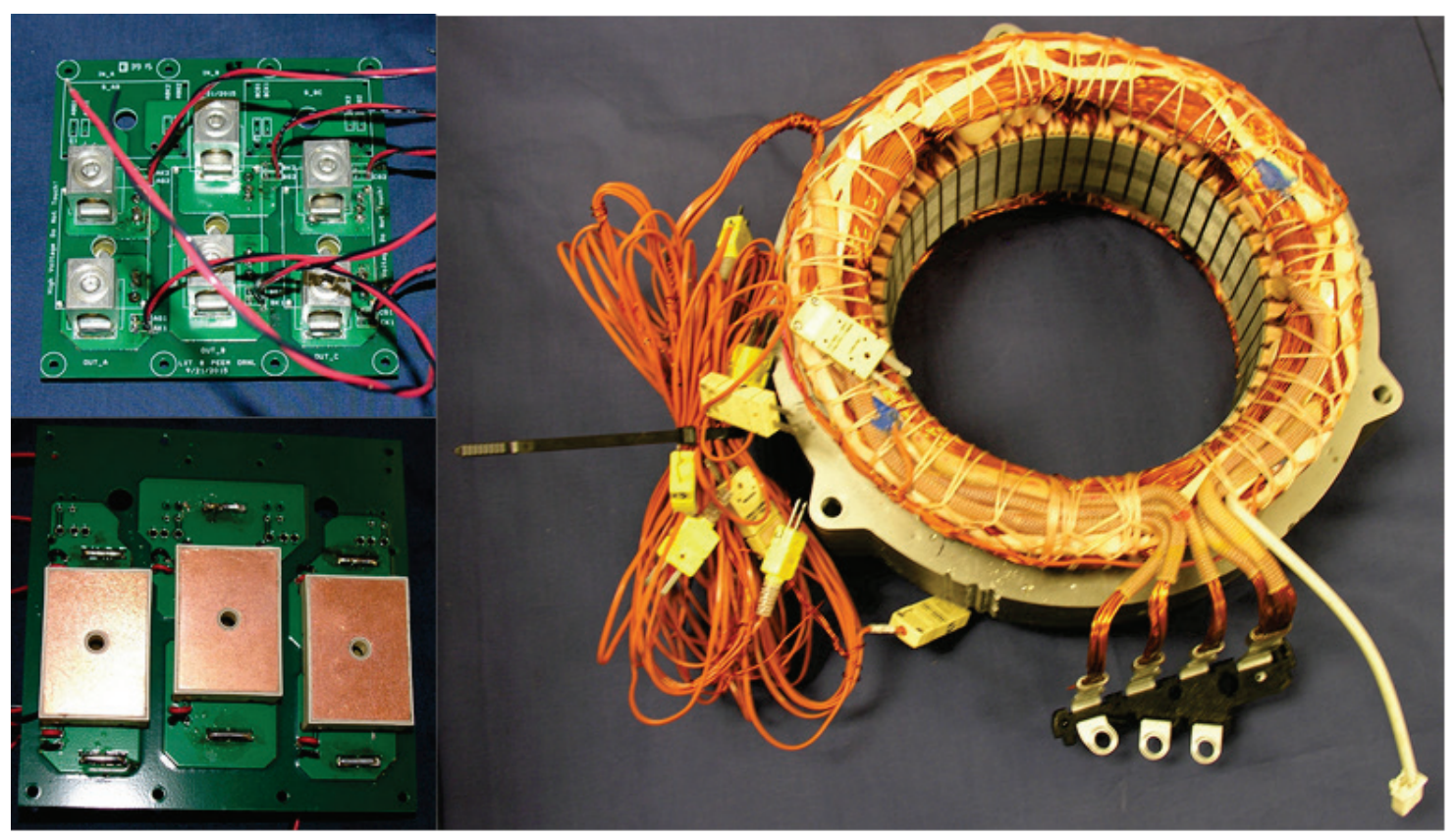

Figure 10: Solid-state ac switch board (top view at top left; bottom view at bottom left) and motor winding used in benchtop test (right).

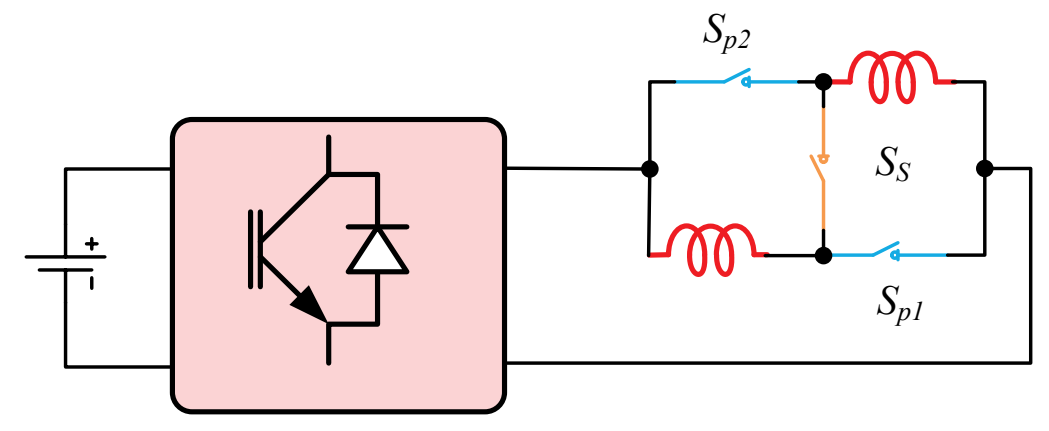

Figure 11: Test circuit diagram with an inverter and three solid-state ac switches. 


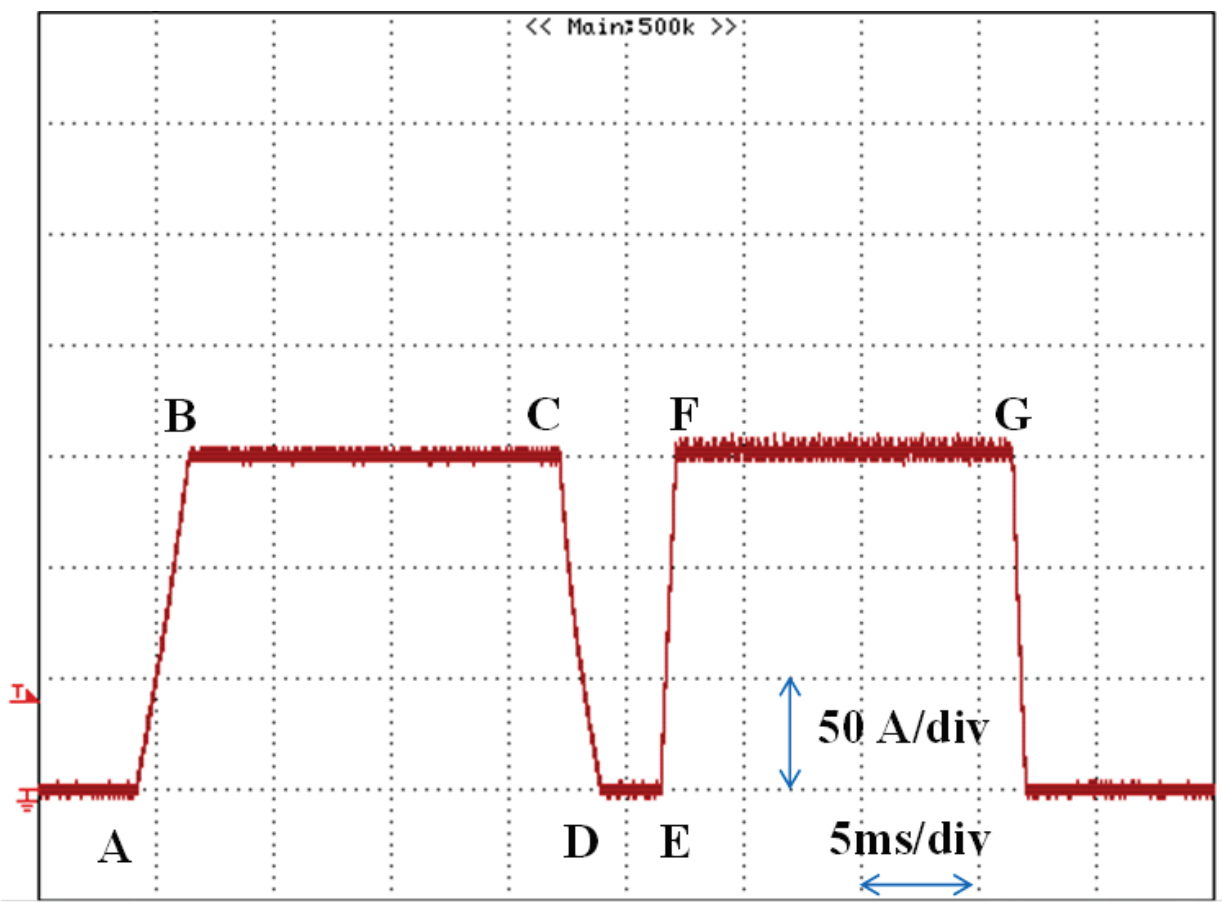

Figure 12: Key test waveform of a series to parallel transient, output current $50 \mathrm{~A} / \mathrm{div}$, time is $5 \mathrm{~ms} / \mathrm{div}$.

\section{Conclusions and Future Direction}

A literature review indicates that most MSR research is ongoing and not on the market. There are different paths to achieving MSR operation, such as switchable winding

Drive cycle simulations show that an MSR solution with three speed ranges reduces drive cycle losses by about $24 \%$ in a combined drive cycle. The loss reductions for different drive cycles are different. The greatest benefit is achieved in the US06 drive cycle (30\% loss reduction). In the UDDS cycle, the MSR design reduces losses by $28 \%$; and in the HWFET drive cycle, it cuts losses by $4.6 \%$. We developed a MSR solution with fewer solid state switches that does not have a battery short-circuit failure mode. It achieves significant loss reduction compared with a fixed winding motor drive and a state-of-the-art approach. A $4.2 \times 4.3 \mathrm{in}$. ac switch PCB based on a low-cost solid-state switch was designed, fabricated, and tested. Initial test results show it can change the motor winding configuration in a fairly short time.

In FY 2016, we will build and test a benchtop prototype to verify the benefits of the novel MSR solution. We will build a final prototype in FY 2017.

For commercialization, in-depth discussions will be held with automotive original equipment manufacturers to maintain their awareness of the advancements made in this motor project

\section{FY 2015 Presentations/Publications/Patents}

1. L. Tang and T. Burress, "Multi-speed-range electric motors," presented at the DOE Vehicle Technologies Program Electric Drive Technologies FY 2015 Kickoff Meeting, Oak Ridge, Tennessee, November 19, 2014.

2. L. Tang and T. Burress, "Multi-speed range electric motor R\&D," presented at the DOE Vehicle Technologies Program Electric Drive Technologies Electrical and Electronics Technical Team Meeting, February 26, 2015.

3. L. Tang and T. Burress, "Multi-speed-range electric motor R\&D," presented at the DOE Vehicle Technologies Office 2015 Annual Merit Review, Washington, DC, June 2015. 


\section{References}

1. D. A. Fulton, "Switch Module for an Electric Machine Having Switchable Stator Windings," US patent application publication, US 2012/0068656 A1, March 22, 2012.

2. M. Krieger and H. Shum, "Electric Motor Having Windings Operable in Parallel and/or Series, and Related Methods," US patent application publication, US 2012/0086380 A1, April 12, 2012. 


\subsection{Inverter R\&D}

Madhu Chinthavali, Principal Investigator

Oak Ridge National Laboratory (ORNL)

National Transportation Research Center

2360 Cherahala Boulevard

Knoxville, TN 37932

Phone: (865) 946-1411

E-mail: chinthavalim@ornl.gov

Susan A. Rogers, DOE EDT Program Manager

Phone: (202) 586-8997

E-mail: Susan.Rogers@ee.doe.gov

Burak Ozpineci, ORNL EDT Program Manager

Phone: (865) 946-1329

E-mail: burak@ornl.gov

Contractor: UT-Battelle, LLC, managing and operating contractor for the Oak Ridge National Laboratory Contract No.: DE-AC05-00OR22725

\section{Abstract/Executive Summary}

The overall objective of this project is to design and develop a high-voltage wide-bandgap (HV-WBG) $30 \mathrm{~kW}$ continuous $55 \mathrm{~kW}$ peak power inverter. WBG devices offer some distinct advantages over silicon ( $\mathrm{Si}$ ) components. Primarily, they can operate at higher junction temperatures. This benefit allows for hotter coolant and smaller heat sinks and can potentially help facilitate air cooling without a sacrifice in performance. Many of the typical components in a commercial inverter cannot withstand the desired operating temperature of WBG devices, i.e., the capacitor and gate driver. Thus the whole inverter must be considered in developing new high-temperature packages. The new concepts developed under this project will increase the power density and decrease the volume and weight for electric-base vehicle traction-drive inverters and will achieve the DOE 2022 weight, volume, and efficiency targets.

\section{Accomplishments}

- Completed evaluation of a 1,200 V, $30 \mathrm{~A}$, trench silicon carbide ( $\mathrm{SiC}$ ) metal-oxide-semiconductor field-effect transistor (MOSFET)

- Completed the design, build, and testing of a $10 \mathrm{~kW}$ WBG-based air-cooled inverter prototype using ORNL's WBG modules

- Completed the design, build, and testing of a $10 \mathrm{~kW}$ WBG-based liquid-cooled inverter prototype using an advanced package built at ORNL

- Completed the design and build of a $30 \mathrm{~kW}$ WBG-based liquid-cooled inverter

\section{Introduction}

There is an increasing need for higher-temperature operation of power electronics in automotive applications. The capability of components to operate reliably at elevated temperatures can enable cost and weight savings by making it feasible to reduce the sizes of heat sinks and eliminate secondary cooling loops. Additionally, devices capable of increased-frequency operation can reduce requirements for passive components, leading to further reductions in cost, weight, and volume. WBG devices, specifically $\mathrm{SiC}$ and gallium nitride semiconductors, are emerging technologies that enable operation at higher temperatures and frequencies, as well as efficiency and reliability improvements. The development of WBG devices promises to help achieve 
these goals, as well as VTO targets. WBG technology assessment performed under this project will help to determine when a viable market introduction of these devices for automotive use will occur. The independent assessment of devices for the automotive industry is carried out to monitor progress and provide data readily when the need arises.

It should be no surprise that none of the electric drive vehicle traction drive systems on the market can meet cost and efficiency goals. Efficiency is achieved by using lower-loss devices and materials, which tend to be expensive even as their quantity levels increase. A case in point is motor lamination steel, lower-loss grades of which are manufactured using novel processes that add more cost.

Problems associated with power electronics for advanced vehicle applications include the following:

- Low efficiency at light load conditions for inverters and converters

- Low current density and device scaling issues for high-power converters

- Lack of reliable higher-junction-temperature devices

- High costs of devices and power modules, especially for WBG and advanced Si devices

- High numbers of components for low-voltage electronics (e.g., gate drivers, controllers, sensors)

- Lack of standardized high-power-density, low-cost power modules for scalable and modular power converters

- Substrates that use expensive ceramics for thermal stability and reliability

- Low-cost, low-loss magnetics and high-temperature films for capacitors

The goal of this research is to reduce the size and weight of power converters to meet the 2015 and 2020 inverter targets. The overall strategy for addressing the limitations of the state of the art is shown in Figure 1.

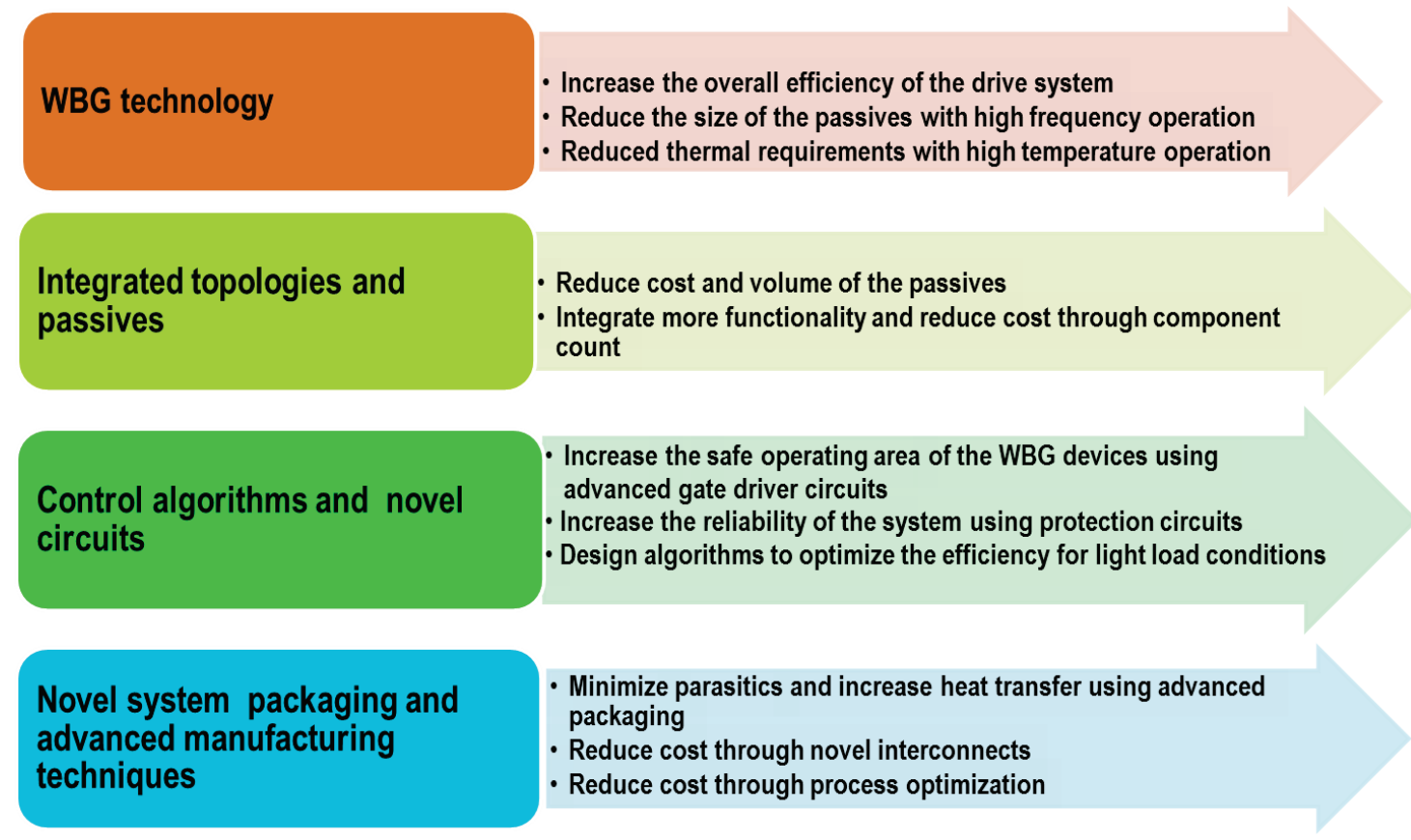

Figure 1: Overall strategy to address limitations of the state of the art.

\section{Approach}

The overall objective of this project is to design and develop a WBG $30 \mathrm{~kW}$ continuous $55 \mathrm{~kW}$ peak power inverter. WBG devices offer some distinct advantages over their Si components. Primarily, they can operate at higher junction temperatures. This benefit allows for hotter coolant and smaller heat sinks and can potentially 
facilitate air cooling without sacrificing performance. Many of the typical components in a commercial inverter cannot withstand the desired operating temperature of WBG devices, i.e., the capacitor and gate driver. Thus the whole inverter must be considered in the development of new high-temperature packages.

The design innovations in this project include the following:

- The design concept uses layers of high-temperature thermal insulating material to separate the lowtemperature components from the high-temperature zone.

- It uses the high-temperature operating capability of WBG devices to enable air cooling and uses newer fast-switching Si insulated gate bipolar transistors for high-temperature-liquid designs.

- The innovative heat sink design minimizes thermal resistance.

- The design is optimized for the most frequently operated points.

These new concepts will increase the power density and decrease the volume and weight for electric-base vehicle traction-drive inverters and will achieve the DOE 2020 weight, volume, and efficiency targets. The specific approach to address the limitations of the state of the art is described in Figure 2.

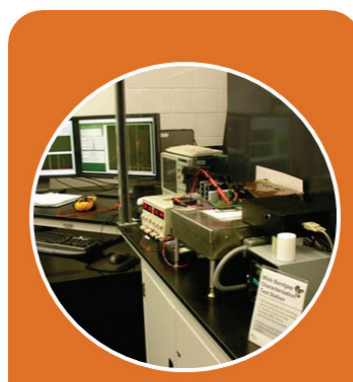

WBG device evaluation

- Static and dynamic characterization: discrete devices

- Single phase test characterization: power modules

- Device models

- Evaluate electrical reliability of the devices

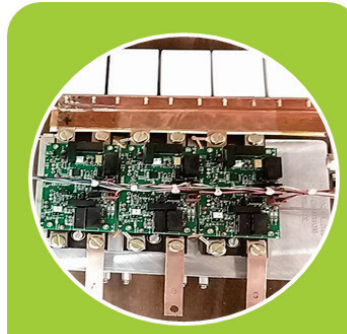

Commercial-modulebased liquid-cooled inverter

Build, test, and evaluate a 30 kW commercial-modulebased WBG inverter

- Use the test results as benchmark

- Scale the inverter as the modules become available

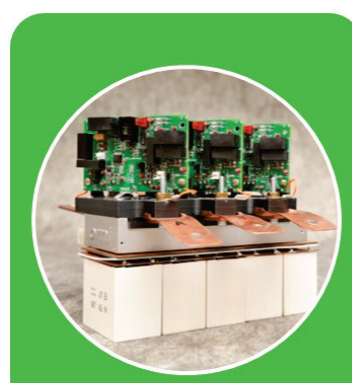

ORNL-module-based liquid-cooled inverter

Build, test, and evaluate a 30 kW ORNL-modulebased WBG inverter

- Compare the results with the commercial module based inverter

- Scale the inverter as the devices become available

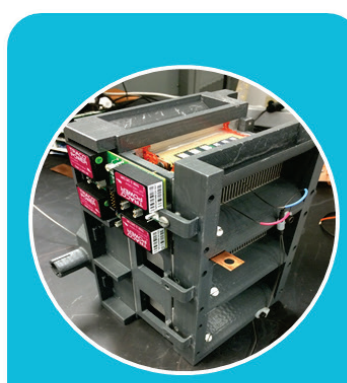

Air-cooled inverter designs

- Build and test a $10 \mathrm{~kW}$ air-cooled WBG inverter

- Compare the results with the liquid-cooled inverters

- Scale the inverter as the devices become available

2022 power electronics targets of $13.4 \mathrm{~kW} / \mathrm{L}$ and $14.1 \mathrm{~kW} / \mathrm{kg}$

Figure 2: Specific approach to address limitations of the state of the art.

\section{Results and Discussion}

\section{Device Testing}

The new WBG devices acquired this year are 1,200 V, 30 A SiC MOSFETs. On-state characteristics and switching energy losses of the devices were obtained over a wide temperature range. All the devices obtained were experimental samples. The static characteristics and switching energy losses of two types of $\mathrm{SiC}$ MOSFETs with a TO-247 packaging case are presented and compared. One is a single 1,200 V, $30 \mathrm{~A} \mathrm{SiC}$ MOSFET with a planar structure); the other is a $1,200 \mathrm{~V}, 30 \mathrm{~A}$ SiC MOSFET with a trench structure.

The output characteristics of the 1,200 V, 30 A planar gate and trench gate SiC MOSFET at $+20 \mathrm{~V}$ gate voltage level for different operating temperatures are shown in Figure 3. The comparison of their on-state resistances is shown in Figure 4. As can be clearly observed, both types of SiC MOSFETs present a positive 
temperature coefficient in on-state resistance, which is beneficial for device paralleling operation. Moreover, although they have the same current and voltage rating, the on-state resistance of the trench gate device is only one-half that of the planar gate device, which indicates the new trench gate SiC MOSFETs can achieve even higher power density.

The dynamic performance of both kinds of SiC MOSFETs was characterized by the ORNL WBG device evaluation facility. Specifically, a universal double pulse power test setup was built to test their switching performance, as shown in Figure 5. The new version of the test setup adds several features: (1) a built-in solidstate circuit breaker for overcurrent and short circuit protection; (2) compatibility with Si/WBG devices featuring various device packages, TO-220, TO-247, and so on; (3) compatibility with different measurement methods (shunt, Pearson, current probe).
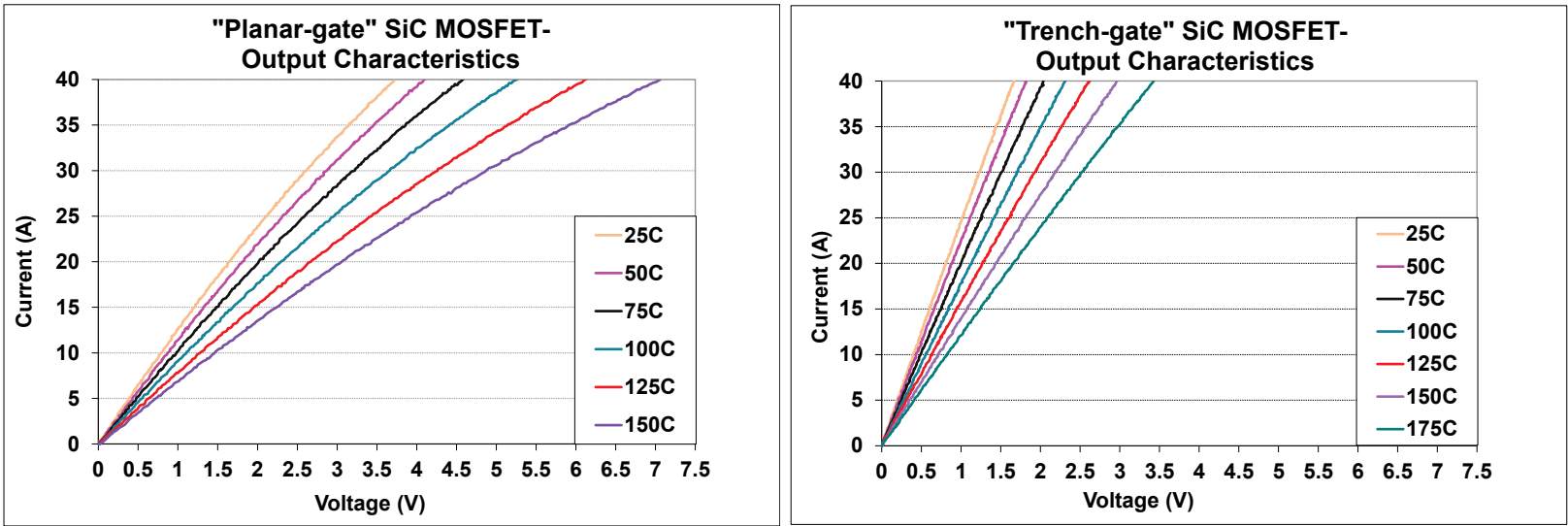

Figure 3. The i-v curves of a $1200 \mathrm{~V}, 30$ A SiC MOSFET.

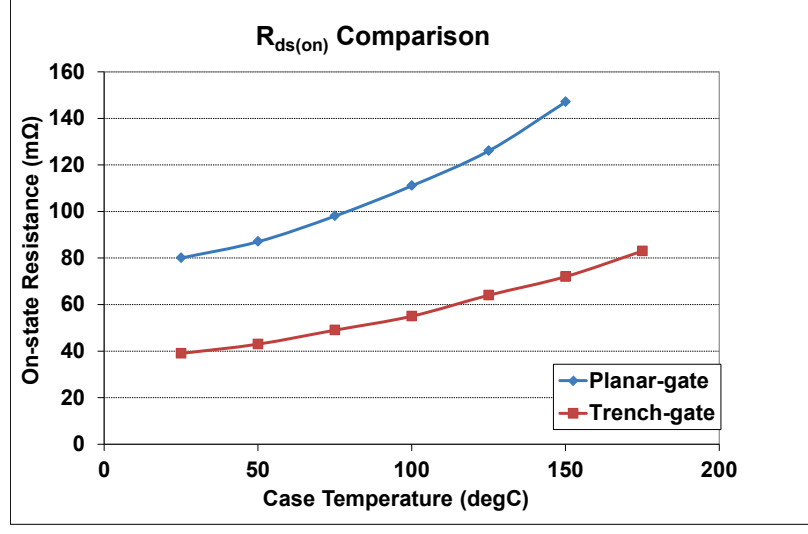

Figure 4. On-state resistances.

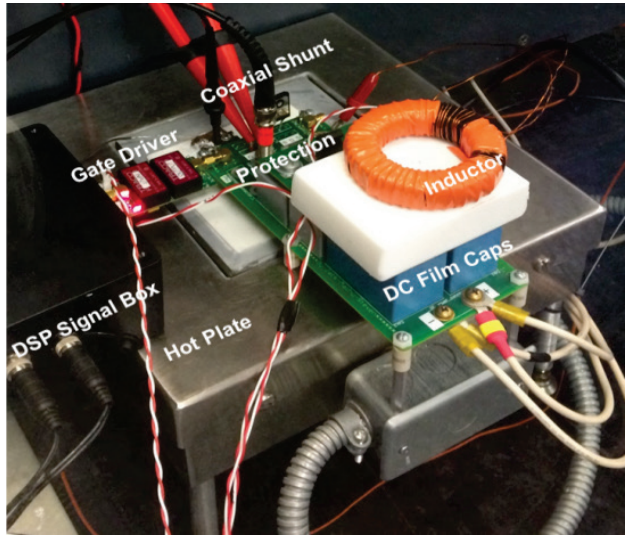

Figure 5. Double pulse test setup.

The turn-on, turn-off energy losses and total energy losses of the planar gate SiC MOSFETs and trench gate $\mathrm{SiC}$ MOSFETs were obtained using a universal double-pulse circuit with a load inductance of $360 \mu \mathrm{H}$, as shown in Figures 6 and 7, respectively. The gate driver used for this testing was a commercial gate driver chip with high sourcing and sinking current capability.

The data for the planar gate $\mathrm{SiC}$ MOSFETs were obtained at $600 \mathrm{Vdc}$ for various currents under different case temperatures from 25 and $175^{\circ} \mathrm{C}$, as shown in Figure 6(a) - (c). As can be observed, the turn-on, turn-off, and total energy losses increased with an increase in current. With a rise in temperature, the turn-on energy loss decreased as the result of a faster switching speed, whereas the turn-off energy loss increased as the result of a slower switching speed. The net result was that the total switching energy loss became somewhat lower at higher temperatures. 


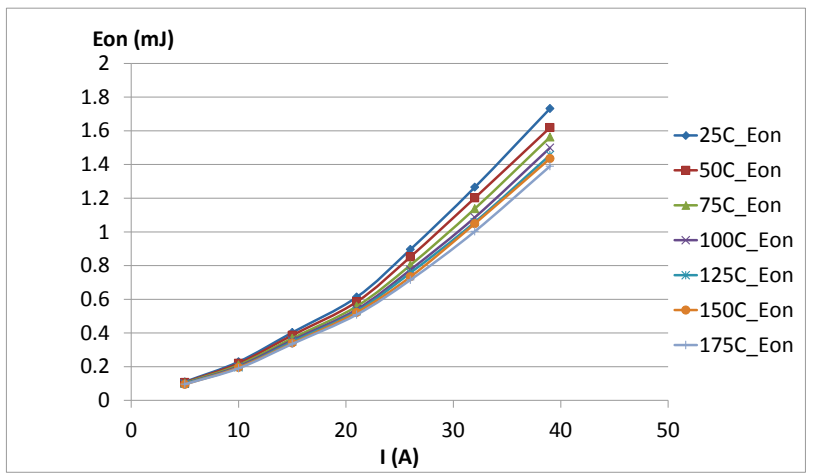

(a) Turn-on loss

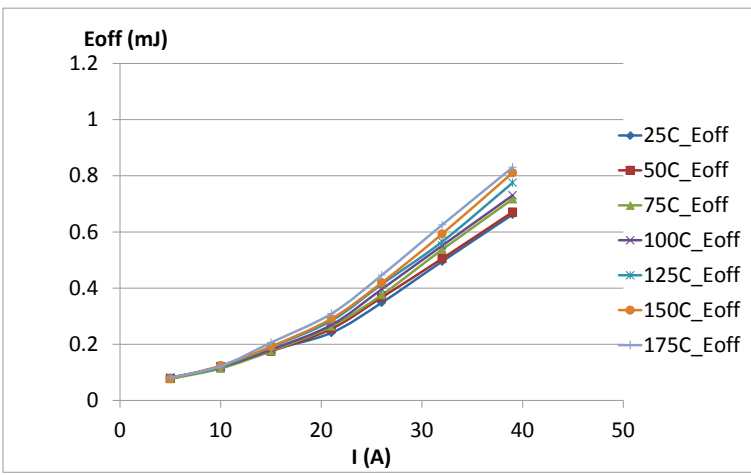

(b) Turn-off loss

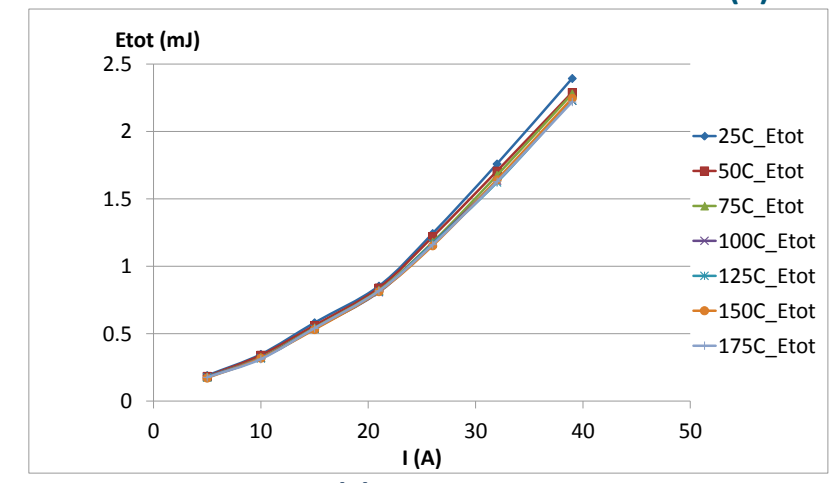

(c) Total loss

Figure 6. Energy losses of planar gate SiC MOSFETs.

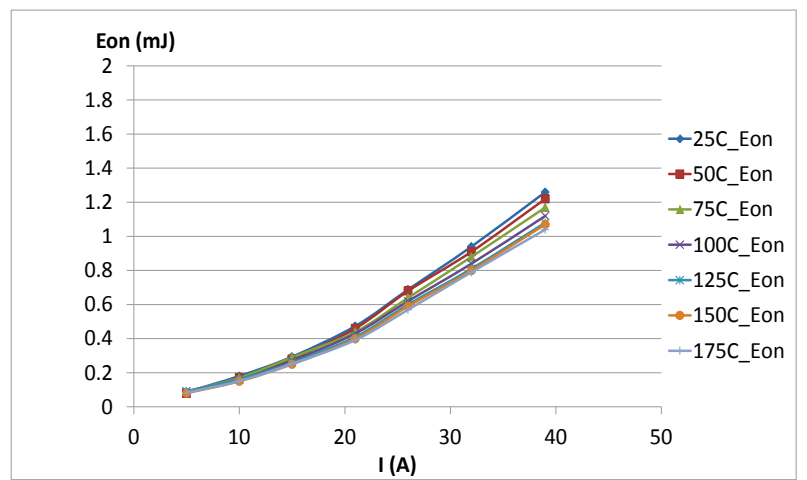

(a) Turn-on loss

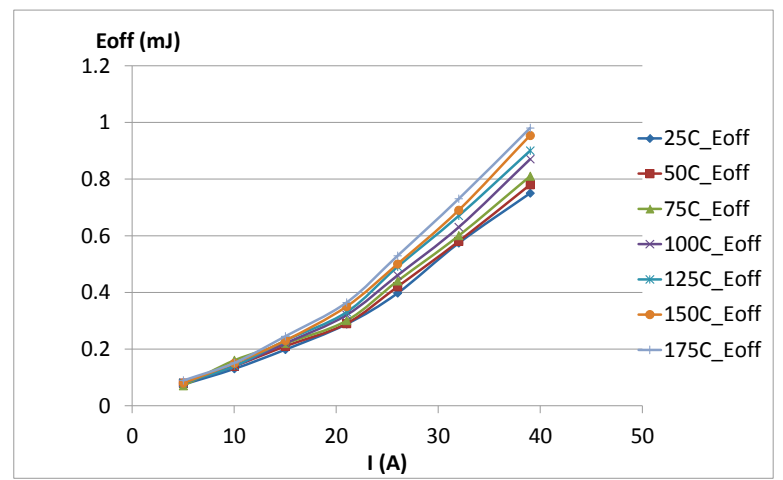

(b) Turn-off loss

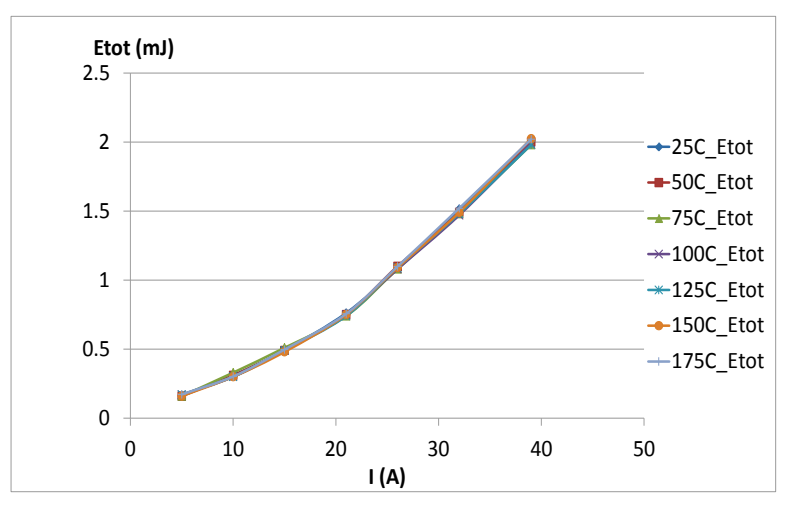

(c) Total loss

Figure 7. Energy losses of trench gate SiC MOSFETs. 
Figures 7(a) - (c) illustrate the turn-on, turn-off, and total energy losses of the SiC MOSFET with trench gate structure under the same testing conditions $\left(600 \mathrm{~V}, 25\right.$ to $\left.175^{\circ} \mathrm{C}\right)$. Compared with the planar gate $\mathrm{SiC}$ MOSFET, the turn-on energy loss was lower because of smaller equivalent junction capacitance and thus faster switching speed; and the turn-off energy loss was nearly the same. Therefore, the total energy loss of the tested trench device was a little lower.

\section{WBG Inverter Design and Development}

Device packages able to withstand high temperatures are essential to take advantage of the high-temperature operating capability of WBG devices. Various organizations are working on high-temperature packaging for high-temperature devices. Several high-temperature packages, which include discrete device packages for power modules, have been reported in the past several years. Novel packaging concepts focus primarily on improving the existing packages, designing new packages using new materials, and/or developing processing techniques for better reliability and performance. Even though the novel packages enable the devices to work at higher temperatures, theoretical advantages such as the current density of WBG devices are not realized because of the interconnects needed for the power module to access the device terminals. In addition, the novel designed packages need further development to be used in full systems.

Other factors that limit system designers from reaping the benefits of WBG technology are low-voltage electronics and passive components. This is because although the packages and the power devices can handle high temperatures, the low-power electronics that drive the power devices, silicon-on-insulator (SOI) -based technologies, are limited to a maximum temperature of $200^{\circ} \mathrm{C}$. Silicon-based electronics are limited to $125^{\circ} \mathrm{C}$ operating temperatures. Although SOI-based electronics can work at up to $200^{\circ} \mathrm{C}$, they are expensive. Hightemperature (over $200^{\circ} \mathrm{C}$ ) electronics have been reported to be feasible; however, they have not been built to demonstrate their performance capabilities. It could be many years before a logic-level high-temperature transistor can be built. This time lag creates a void in the power module industry, especially for intelligent power module products, which include the electronics inside the module.

Similarly, the passive components in an inverter have low operating temperatures and cannot be operated in close proximity to high-temperature WBG devices. This situation leads to an increase in the volume and a reduction in the power density of the system. High-temperature passive components are currently being developed to address the requirement for high-temperature-operation. However, as with the electronic components, they will be much more expensive than the low-temperature components.

To address these problems, a system-level approach for packaging design needs to be developed. Complex 3-dimensional packaging structures with integrated interconnects can reduce the required assembly steps and increase the power densities of power electronic systems. Recent advancements in additive manufacturing (AM) promise an exciting future trend that will allow WBG technology to make inroads into the power electronics industry. AM techniques enable the development of complex 3-dimensional geometries that will result in size and volume reductions at the system level by integrating low-temperature components with hightemperature active devices and reducing the amount of material needed to build the heat exchangers in inverters. ORNL has developed expertise in AM in the past few years. ORNL's PEEM team recognized the potential of this technology for power electronics system packaging and took a first step toward achieving a completely printed inverter concept.

A $10 \mathrm{~kW}$ all-SiC inverter incorporating an aluminum-based printed power module with an integrated cooling system and a printed plastic lead frame was built using AM techniques. This is the first air-cooled inverter prototype built using AM. The design and development of the inverter and characterization of a hightemperature 1,200 V, $100 \mathrm{~A}$ all-SiC module are discussed in the following sections.

ORNL worked with the National Renewable Energy Laboratory (NREL) in developing the air-cooled inverter to further optimize the thermal design. The air-cooled inverter developed in FY 2013 was redesigned using thermal simulations from NREL. The initial inverter size was reduced by $33 \%$ through fin design optimization. A balance-of-plant analysis is currently being conducted to establish the feasibility of air cooling at the system level. The new module design has been fabricated and tested for heat transfer. The design was modified by ORNL and redesigned with 1,200 V, 100 A MOSFET and Schottky diodes. The module layout and fabricated prototype are shown in Figure 8. 


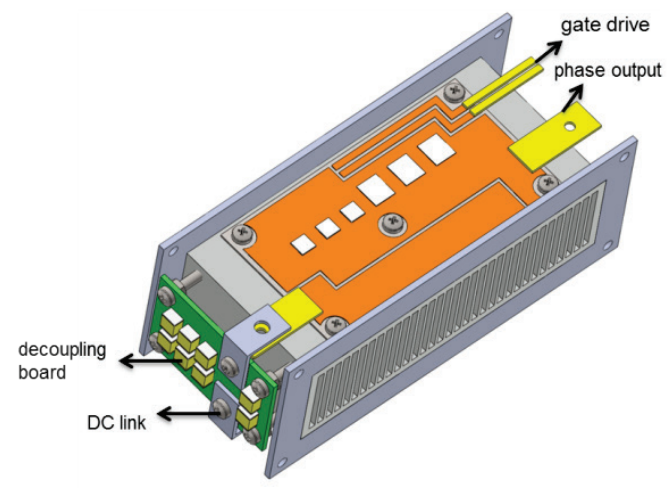

(a) Module layout

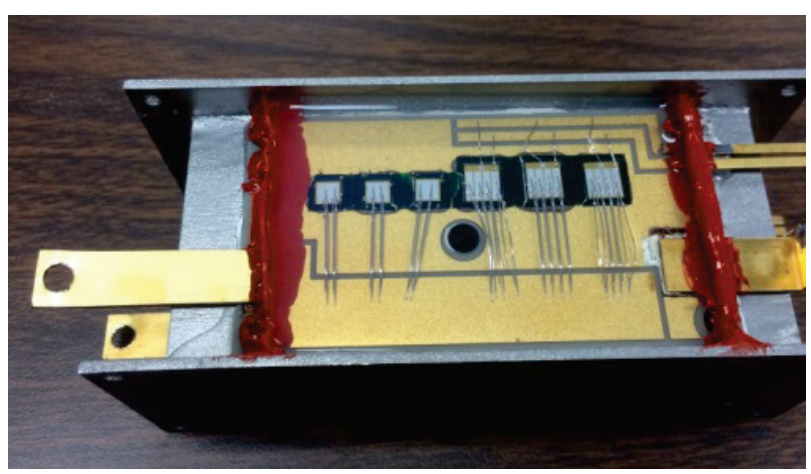

(b) Fabricated module

Figure 8. Air-cooled inverter module.

The switching performance of the power module was evaluated by a double pulse power test setup, as shown in Figure 9. The switching loss with $400 \mathrm{Vdc}$ bus voltages and $10 \mathrm{ohm}$ gate resistance is summarized in Figure 10. The switching speed of the developed module is much slower than that of commercial discrete SiC devices because of a large equivalent gate input capacitance and gate resistance. The slow switching speed also results in a high switching loss, as shown in Figure 10. Also, the turn-on, turn-off, and total switching losses increase linearly with the rise of load current. The switching speed can be pushed faster by using a small gate resistance to obtain low power loss and thus high power density, although the switching stress and associated electromagnetic noise will be a concern for continuous operation. Given that the main goal of this project is to verify the improved thermal performance of the 3-dimensional printed air-cooled power module structure, a relatively large power loss is preferable to demonstrate its heat dissipation capabilities.

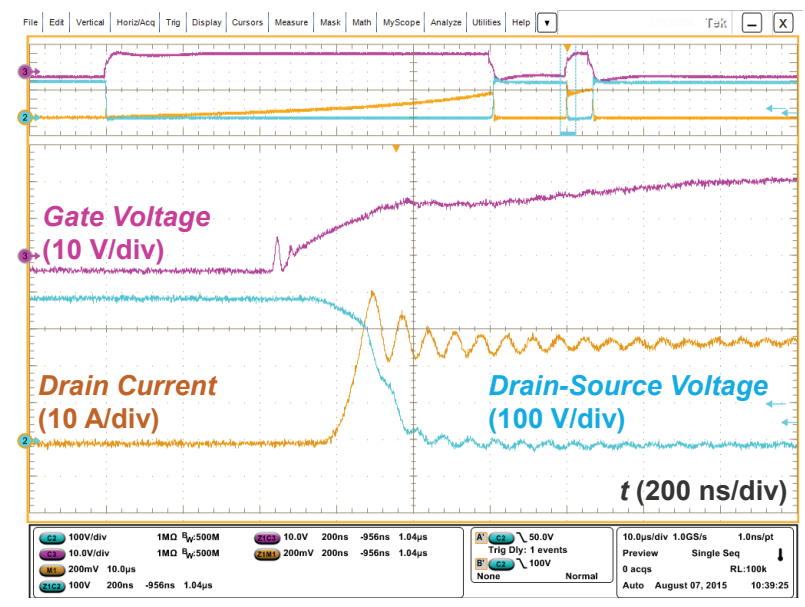

(a) Turn-on transient

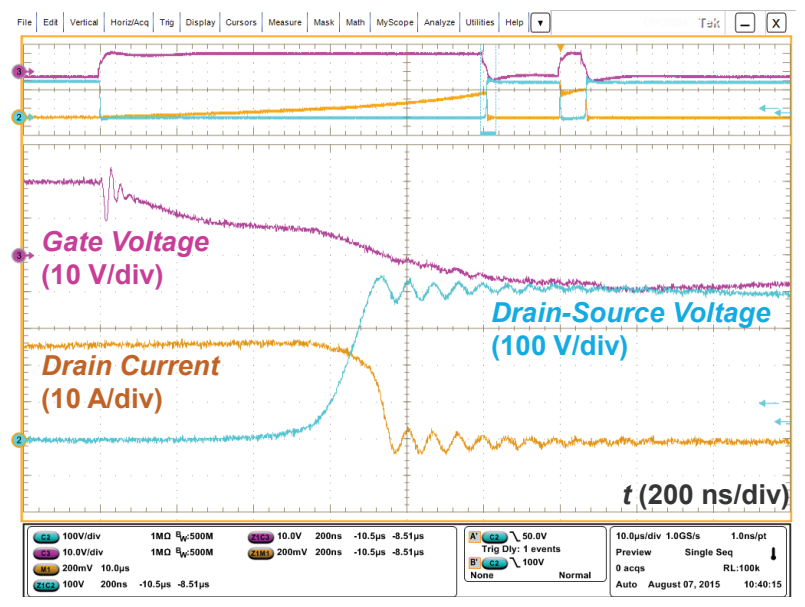

(b) Turn-off transient

Figure 9. Air-cooled module switching performance.

Three sets of phase-leg power modules with 3-dimensional printed heat sinks were fabricated to build a 3-phase voltage source inverter. A dc link bus bar with six $40 \mu \mathrm{F}$ film capacitors was designed to obtain a high form factor. In addition, an air duct was designed and installed at the input and output of the heat sinks for a low air pressure drop. The inverter structure and final assembly are shown in Figure 11(a) and Figure 11(b), respectively. 


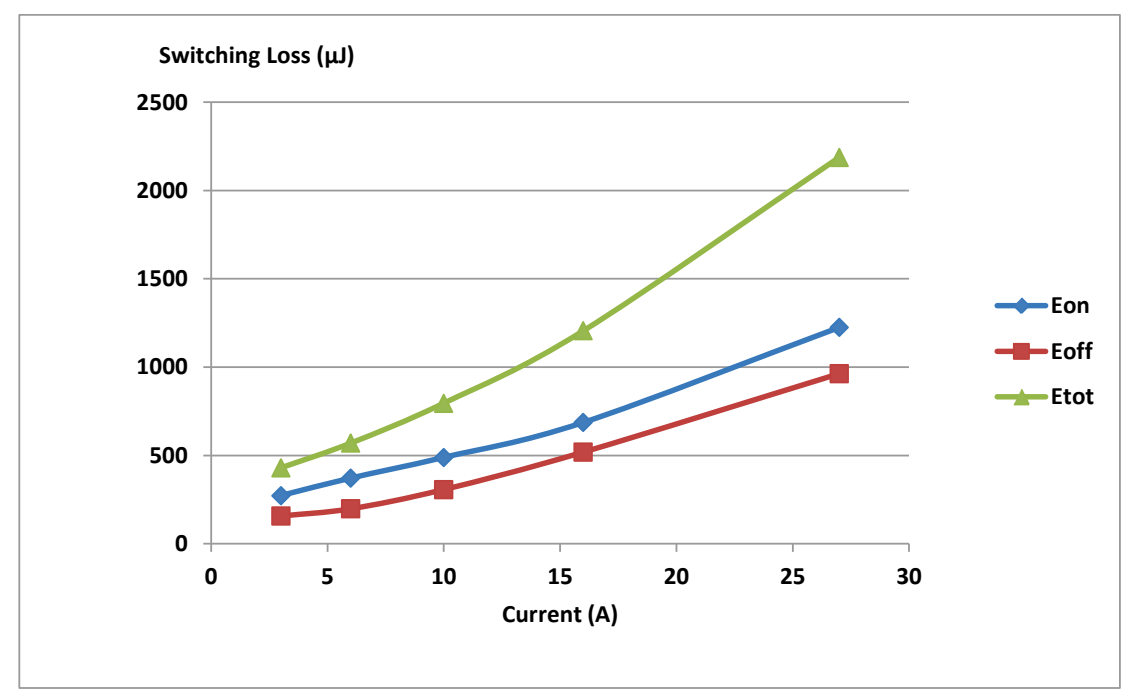

Figure 10. Air-cooled module switching loss with $400 \mathrm{~V} \mathrm{dc}$ bus voltage.

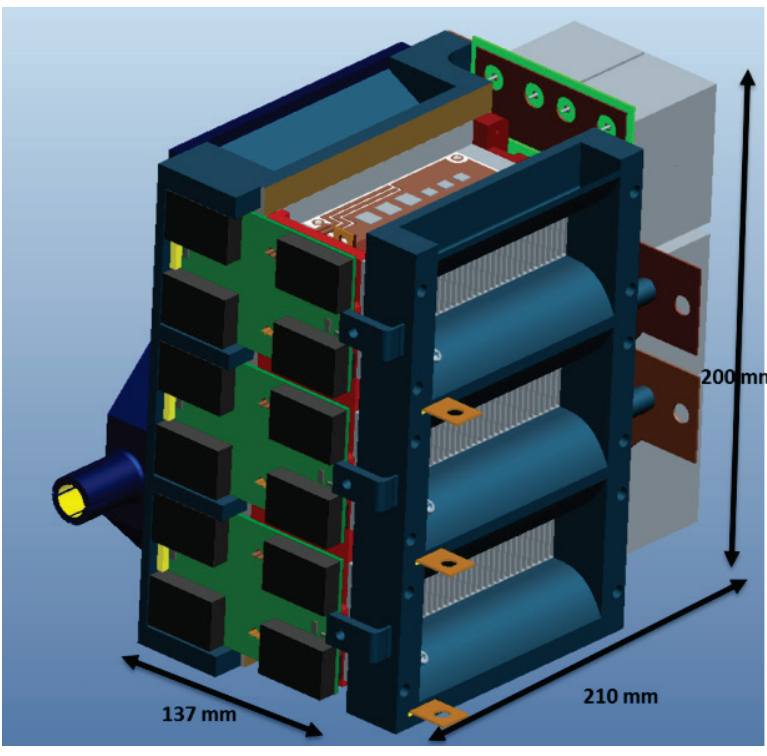

(a) Inverter structure

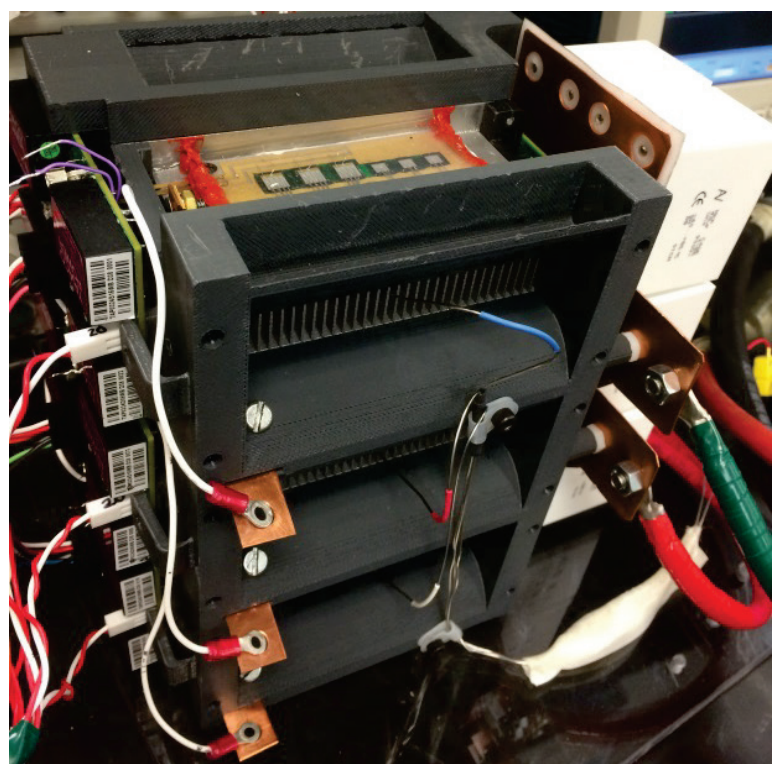

(b) Assembled inverter

Figure 11. Air-cooled inverter assembly.

The assembled 3-phase air-cooled inverter was tested under different dc bus voltages and load conditions. Results for continuous operation with a de bus voltage of $350 \mathrm{~V}$ and power of $13 \mathrm{~kW}$ are shown in Figure 12 . The efficiency of the inverter is summarized in Figure 13. Under light load conditions, the efficiency is relatively low as a result of the high switching loss. As the load current increases, the efficiency gradually increases to $98 \%$. 


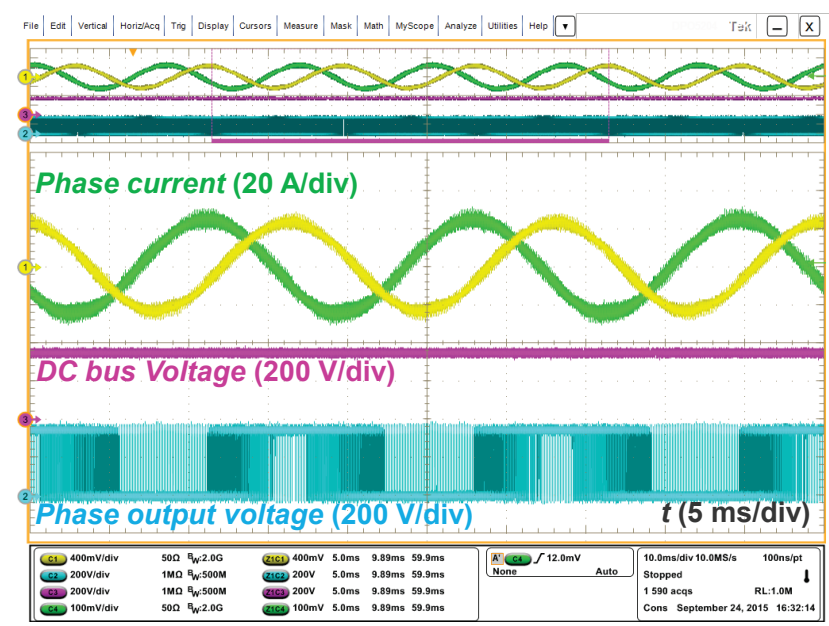

(a) Inverter structure

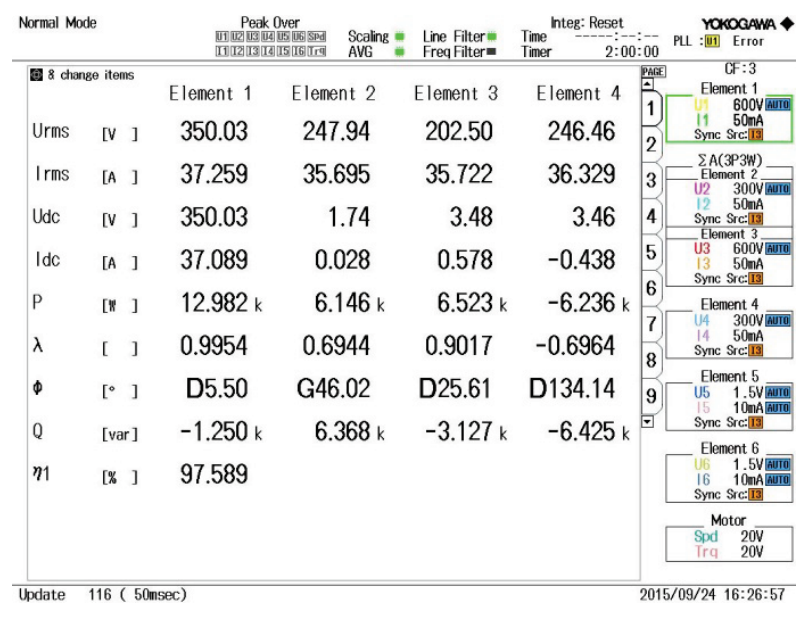

(b) Assembled inverter

Figure 12. Air-cooled inverter testing results.

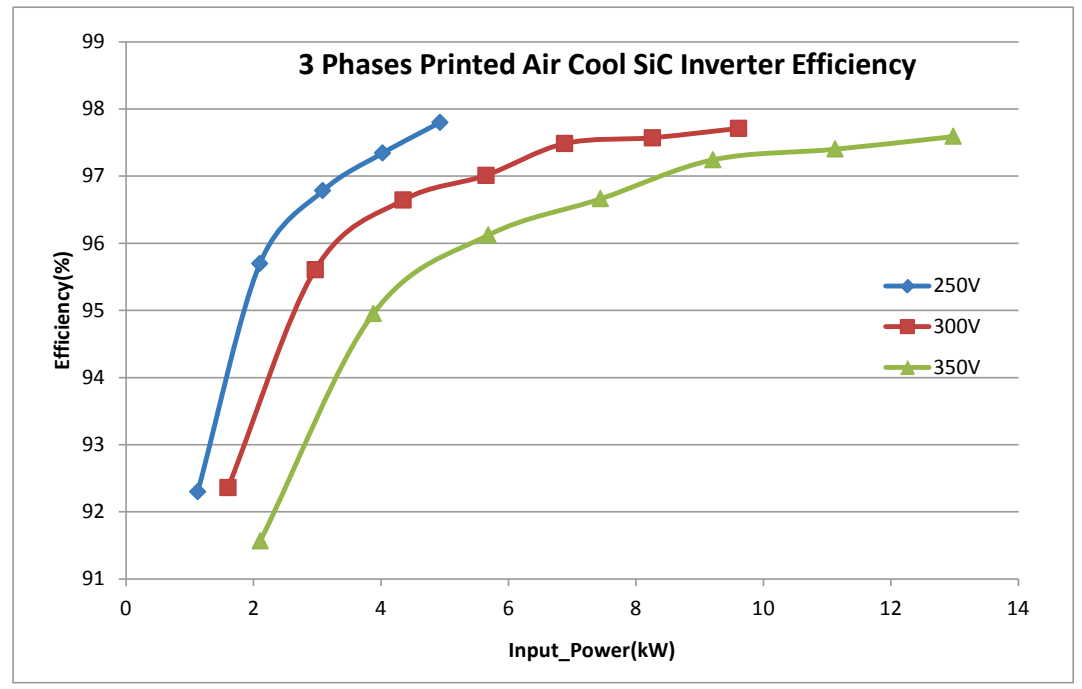

Figure 13. Efficiency of the air-cooled inverter.

The $30 \mathrm{~kW}$ all-SiC inverter was designed using a commercially available 1,200 V, 100 A SiC MOSFET-based module. The layout of the inverter is shown in Figure 14. The total inverter volume is $3.6 \mathrm{~L}(226 \times 224 \times$ $73 \mathrm{~mm}$ ). Commercially available gate drivers from Rohm were used. The gate driver has galvanic isolation up to 3,000 Vrms and integrated overcurrent protection, undervoltage lockout, and temperature feedback. The performance of the module was evaluated before the inverter was built. The cooling system for this prototype is a single-sided cooling commercially available cold plate. The modules were mounted on the cold plate with thermal grease as the heat transfer medium from the lower sides of the power modules. This prototype model will be packaged as shown in Figure 14, with the controls and capacitors packaged close to the heat sink. The capacitors used in this design are not a brick type but are small individual capacitors in series to ensure better cooling and reduce costs. 

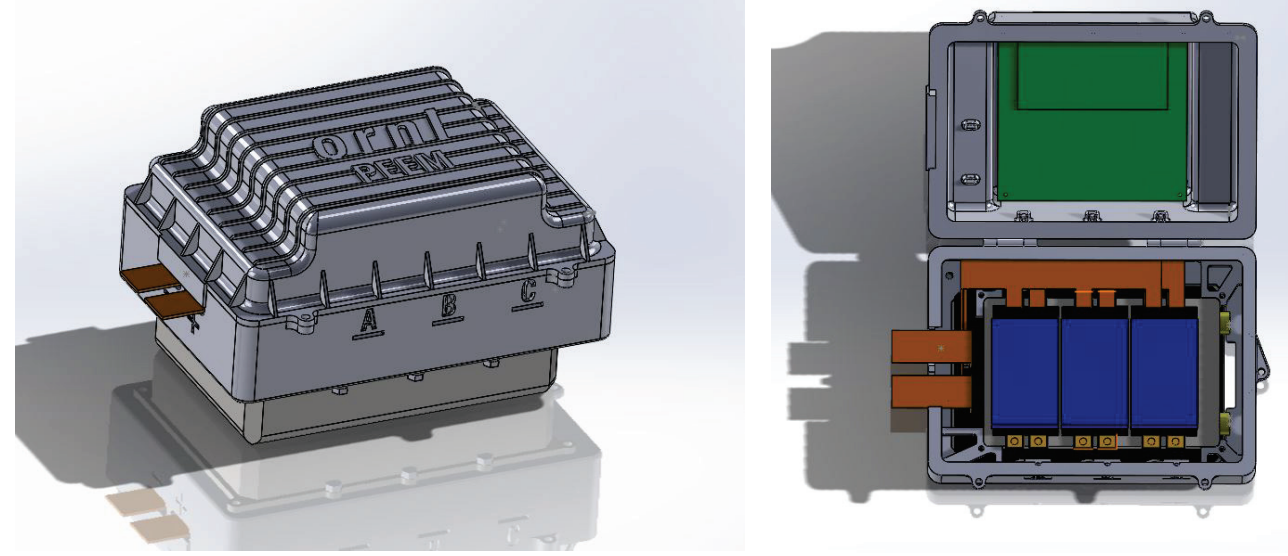

Figure 14. The $10 \mathrm{~kW} \mathrm{SiC} \mathrm{inverter} \mathrm{layout.}$

The final inverter assembly inverter is shown in Figure 15. For this test, the dc-link voltage was fixed at nominal operating voltage $(325 \mathrm{~V})$ to the maximum bus voltage $(450 \mathrm{~V})$. The load resistance was set to the minimum value, and the current was controlled by changing the modulation index. The coolant was set at $20^{\circ} \mathrm{C}$ at a flow rate of $1.5 \mathrm{gpm}$. The open-loop frequency of operation and the pulsed width modulation frequency were fixed, and the current command was varied for a particular dc-link voltage. The command current was increased in steps without exceeding the power rating of the inverter or of the load. The coolant temperature was changed to $60^{\circ} \mathrm{C}$, and data were recorded for a wide range of current and switching frequencies.

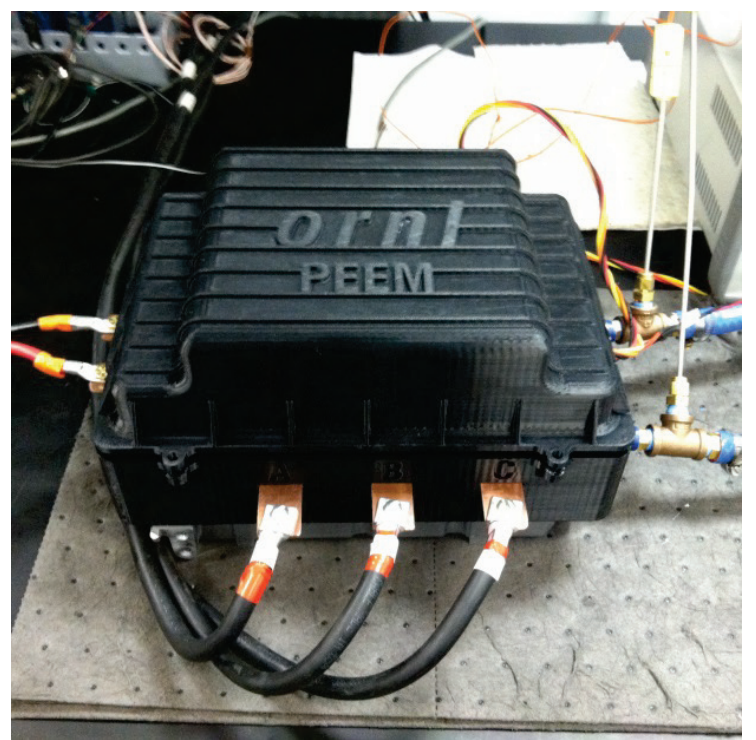

(a) Inverter structure

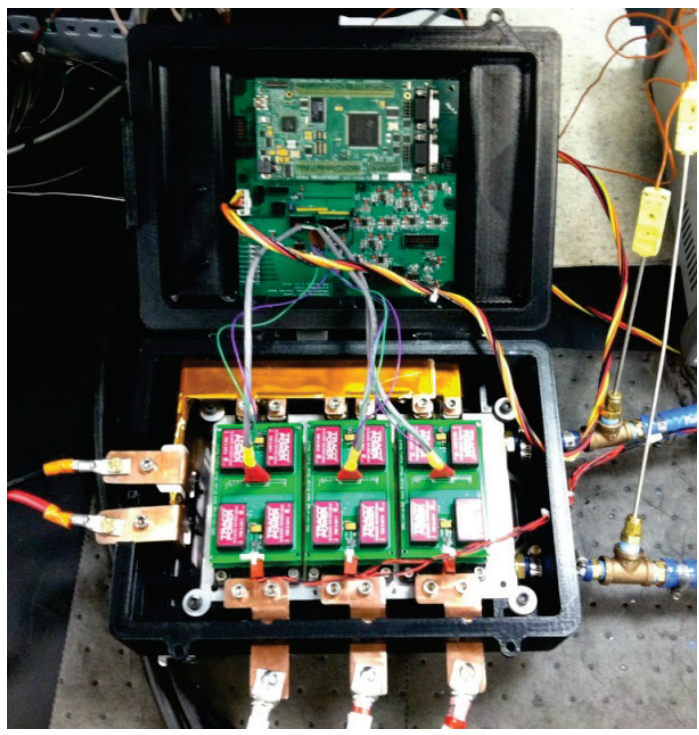

(b) Assembled inverter

Figure 15. Liquid-cooled inverter assembly.

The experimental waveforms and results for $400 \mathrm{Vdc}$ bus voltage and $27 \mathrm{~kW}$ active power operation are shown in Figure 16. 


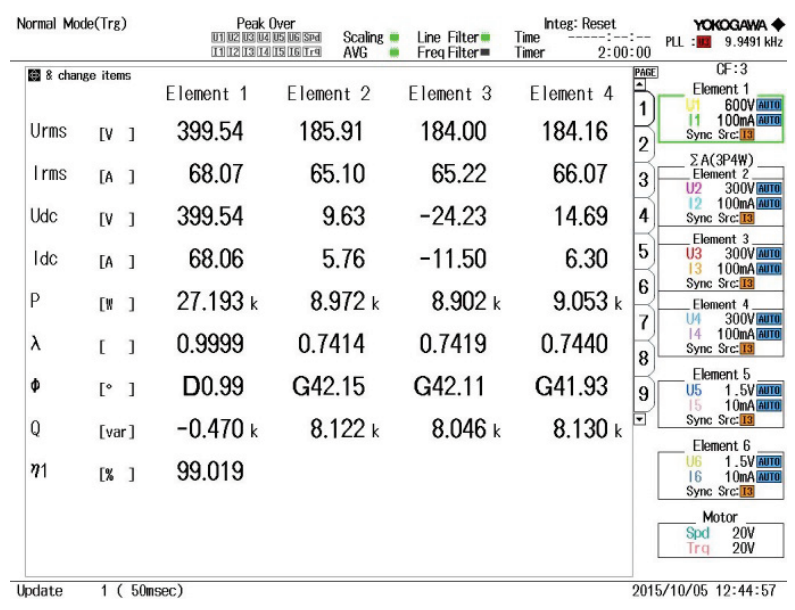

(a) Power analyzer data

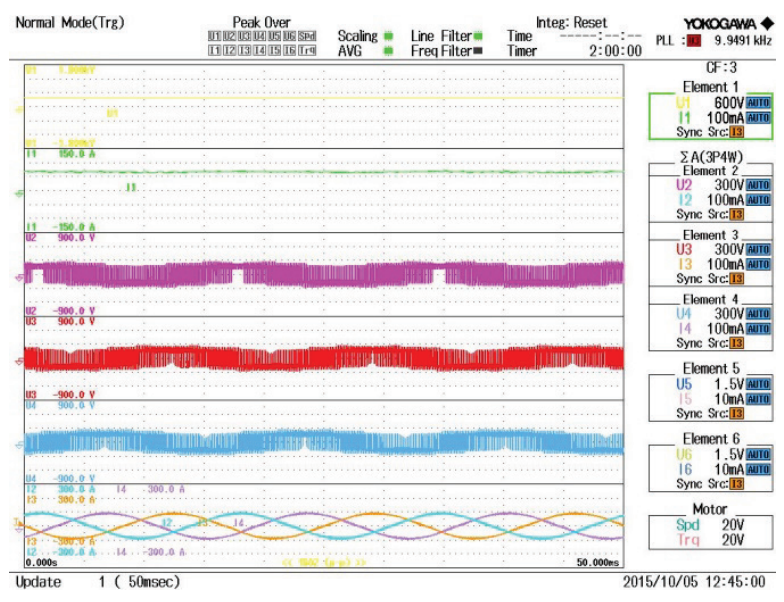

(b) Experimental waveforms

Figure 16. Experimental waveforms of $30 \mathrm{~kW}$ SiC inverter with $400 \mathrm{~V}$ dc-link operation.

Figure 17 shows the efficiency-versus-output-power plot for several operating conditions, comparing efficiencies at different voltages. Inverter efficiencies are higher at $450 \mathrm{~V}$ than at the $325 \mathrm{~V}$ operating condition, as expected.

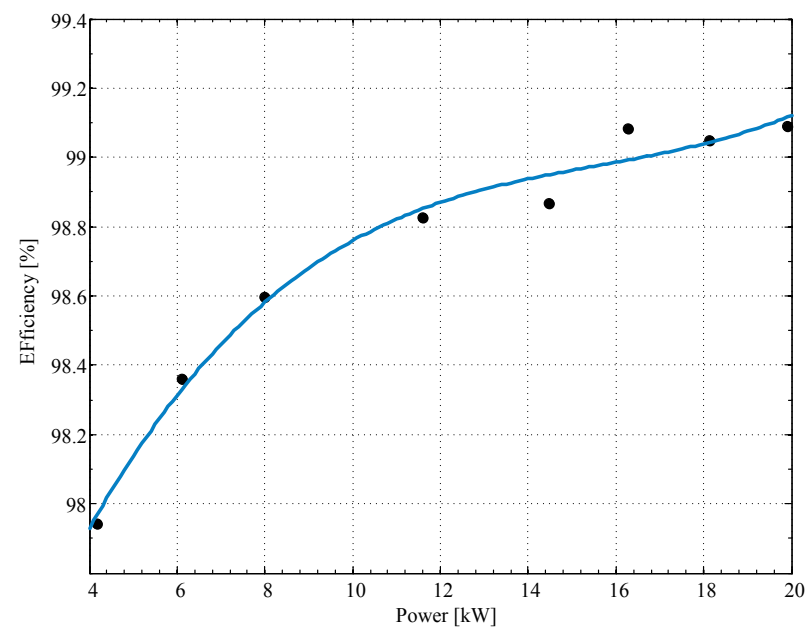

(a) Power vs. efficiency curve (@350 v)

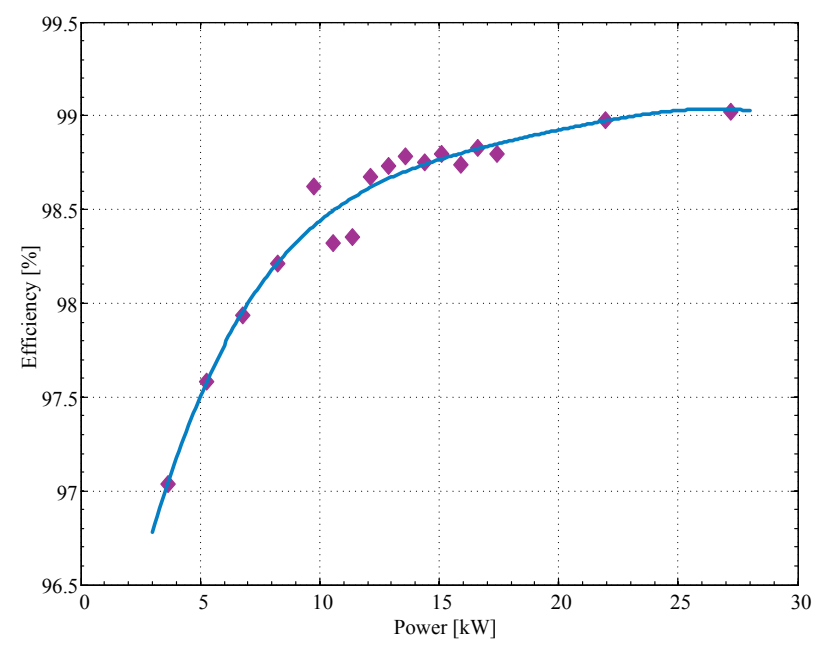

(b) Power vs. efficiency curve (@400 v)

Figure 17. Inverter efficiency vs. output power.

\section{Conclusions and Future Direction}

WBG device evaluation will continue until the technology transitions to industry. The design, development, and testing of an air-cooled $\mathrm{SiC}$ inverter with a printed power module AM heat sink was presented. The total operating power density of the laboratory prototype inverter was $\sim 2.26 \mathrm{~kW} / \mathrm{L}$, and the average operating efficiency of the inverter for a wide range of operating conditions was around $98 \%$. However, based on the design, the power density could potentially be four times greater for higher power with the same power module. This prototype is the first air-cooled inverter built using AM techniques. The power density of the commercial module based on a $30 \mathrm{~kW}$ all SiC inverter built at ORNL is $\sim 4.96 \mathrm{~kW} / \mathrm{L}$.

The inverter test results obtained during FY 2015 will be used as a benchmark for next-generation higherpower inverters to be built using ORNL's WBG package. The results obtained show that if the inverter is 
scaled to $30 \mathrm{~kW}$, it will meet the 2020 VTO targets. They also show that WBG technology will aid in achieving U.S. DRIVE targets for volume, efficiency, power density, and system costs.

\section{FY 2015 Presentations/Publications/Patents}

1. M. Chinthavali, C. Ayers, S. Campbell, and R. Wiles, "A 10-kW SiC inverter with a novel printed metal power module with integrated cooling using additive manufacturing," 2014 IEEE Workshop on Wide Bandgap Power Devices and Applications (WiPDA), pp. 48-54, Knoxville, Tennessee, October 13-15, 2014,. 


\title{
3.2 Innovative Technologies for Converters and Chargers
}

\author{
Gui-Jia Su, Principal Investigator \\ Oak Ridge National Laboratory (ORNL) \\ National Transportation Research Center \\ 2360 Cherahala Boulevard \\ Knoxville, TN 37932 \\ Phone: (865) 946-1330 \\ E-mail: sugj@ornl.gov
}

Susan A. Rogers, DOE EDT Program Manager

Phone: (202) 586-8997

E-mail: Susan.Rogers@ee.doe.gov

Burak Ozpineci, ORNL EDT Program Manager

Phone: (865) 946-1329

E-mail: burak@ornl.gov

Contractor: UT-Battelle, LLC, managing and operating contractor for the Oak Ridge National Laboratory Contract No.: DE-AC05-00OR22725

\section{Abstract/Executive Summary}

- The overall objective of this multiyear project is to develop low-cost, high-efficiency, high-powerdensity all-wide bandgap (WBG) dc-dc converters and onboard chargers (OBCs). The aim is to reduce charger converter cost by $50 \%$ and weight and volume by a factor of 2 compared with the state of the art and provide charger efficiency of more than $96 \%$.

- A major task for FY 2015 was to design, build, and test an all-SiC integrated OBC at the level 2 charging power of $6.6 \mathrm{~kW}$. To this end, a $100 \mathrm{~kW}$ segmented traction inverter using commercial $\mathrm{SiC}$ metal-oxide-semiconductor field-effect transistors (MOSFETs) and 3-dimensional (3D) printed components was designed, built, and tested. The isolated $\mathrm{SiC}$ charger dc-dc converter developed in FY 2014 was then integrated with the traction inverter to provide an integral onboard charging functionality. Testing and evaluation of the integral onboard charging functionality was successfully completed at power levels up to $6.8 \mathrm{~kW}$.

- Another task for FY 2015 was to develop a gallium nitride $(\mathrm{GaN})$-based charger dc-dc converter. A $6.8 \mathrm{~kW}$ charger converter was designed using the GaN Systems GaN transistor GS66516T, highvoltage heavy copper printed circuit board (PCB) power planes, low-voltage (14 V) high-current PCB power planes, and a planar transformer. A prototype will be built and tested in FY 2016.

\section{Accomplishments}

- Completed the design, fabrication, and testing of a reconfigurable $\mathrm{SiC}$ traction inverter for use in the development of all-WBG integrated OBCs of different topologies. The traction inverter can be configured as either a $100 \mathrm{~kW}$ segmented inverter or a dual 3-phase inverter, each rated at $50 \mathrm{~kW}$.

- Completed the integration and testing of a $6.8 \mathrm{~kW}$ all-SiC integrated OBC using the $100 \mathrm{~kW} \mathrm{SiC}$ segmented traction inverter and the isolated $\mathrm{SiC}$ charger dc-dc converter. It showed a peak efficiency of $96.5 \%$ when charged from a $240 \mathrm{~V}$ source and $92.6 \%$ when charged from a $120 \mathrm{~V}$ source, and a 2\% point improvement over the silicon-based counterpart developed in FY 2013.

- Completed a design for a $6.8 \mathrm{~kW}$ charger converter using GaN Systems GaN transistors, high-voltage heavy copper PCB power planes, low-voltage (14 V) high-current PCB power planes, and a planar transformer. The design has a high power density of $7.1 \mathrm{~kW} / \mathrm{L}$. 
- In collaboration with Aegis Technology, completed fabrication of an advanced magnetic material core set of an E-shaped core and a plate using ORNL's additive manufacturing capability and Aegis Technology's nanocomposite magnetic powders.

\section{Introduction}

Most current plug-in electric vehicles (EVs) employ a stand-alone $\mathrm{OBC}$ to charge the propulsion battery. However, a stand-alone $\mathrm{OBC}$ is not cost-effective because of its large number of components. Moreover, its performance in terms of weight, volume, and efficiency is limited by the capabilities of existing semiconductor and magnetic materials. Bulky and expensive passive components (inductors, capacitors, and transformers) are needed in OBCs because (1) silicon switches constrain switching frequencies to less than $100 \mathrm{kHz}$ at power levels of several kilowatts, and (2) inductors and transformers based on soft ferrite magnetic materials further limit power density and efficiency because of low saturation flux densities $(\sim 0.3 \mathrm{~T})$ and high core losses at high frequencies. As a result, OBCs (1) add significant cost $(\sim \$ 106 / \mathrm{kW}) ;(2)$ have low power-density and specific-power numbers $(\sim 0.6 \mathrm{~kW} / \mathrm{kg}, \sim 0.8 \mathrm{~kW} / \mathrm{L})$; (3) are relatively inefficient (85-93\%); and (4) are unidirectional (can charge the battery but are incapable of vehicle-to-grid support, a highly desirable function in future smart grids).

The problems of existing silicon-based OBC technology are addressed in this multiyear project through overcoming the limitations of existing semiconductor and magnetic materials by using WBG devices, advanced magnetic materials, and novel integrated charger topologies and control strategies to significantly increase power density, specific power, and efficiency at lower cost. Emerging WBG devices-including those made with $\mathrm{SiC}$ and $\mathrm{GaN}$ - and advanced soft magnetic materials enable significant improvements in ac-dc and dc-dc converters, major components of OBCs. Their ability to operate with enhanced efficiency over higher frequencies and temperatures minimizes requirements for the passive components and reduces cooling demands. In addition, a novel control strategy developed under this project and reported in the FY 2014 annual report was shown to reduce the dc link capacitance in the ac-dc stage by $60 \%$. Because currently passive components contribute more than $30 \%$ to the charger cost, weight, and volume in state-of-the-art silicon-based technology, the approach proposed in this project provides enabling technologies to produce low-cost, light, compact, and highly efficient OBCs and converters.

\section{Approach}

Our strategy to address the problems of state-of-the-art OBCs and dc-dc converters is multifold:

- Push the envelope on functional integration of the traction drive, $14 \mathrm{~V} \mathrm{dc}$-dc converter, and OBC.

- Take up the challenge of introducing WBG materials, specifically GaN, into automotive applications to determine what performance, packaging, cost, and efficiency benefits can be gained.

- Perform analysis, modeling, and simulation that lead to a functional prototype meeting VTO OBC specific power, power density, and efficiency requirements while significantly reducing the current cost levels.

- Design, build, test, and demonstrate prototypes.

- Work with U.S. DRIVE to develop insights and lessons learned from the automotive community pertinent to dc-dc converters and OBCs.

- Collaborate with industry stakeholders, universities, and other national laboratories to maximize the impact of this work.

Three technical approaches based on converter topology, advanced semiconductor and magnetic materials, and control strategy are being pursued. First, in power conversion topology, integrated bidirectional WBG OBCs are being developed that (a) provide galvanic isolation; (b) provide an integrated function for dc-dc conversion of high voltages to $14 \mathrm{~V}$; and (c) use soft switching at the dc-dc stage to reduce electromagnetic interference (EMI) and improve efficiency. Figure 1 shows an integrated dc-dc converter and charger architecture 
consisting mainly of an ac filter, a WBG front active converter, a dc bus capacitor, and a WBG isolation converter. The isolation converter integrates the functions for charging both the high-voltage traction battery and the $14 \mathrm{~V}$ battery for vehicle accessory loads. It includes a high-frequency transformer and dc filters as well as WBG switches.

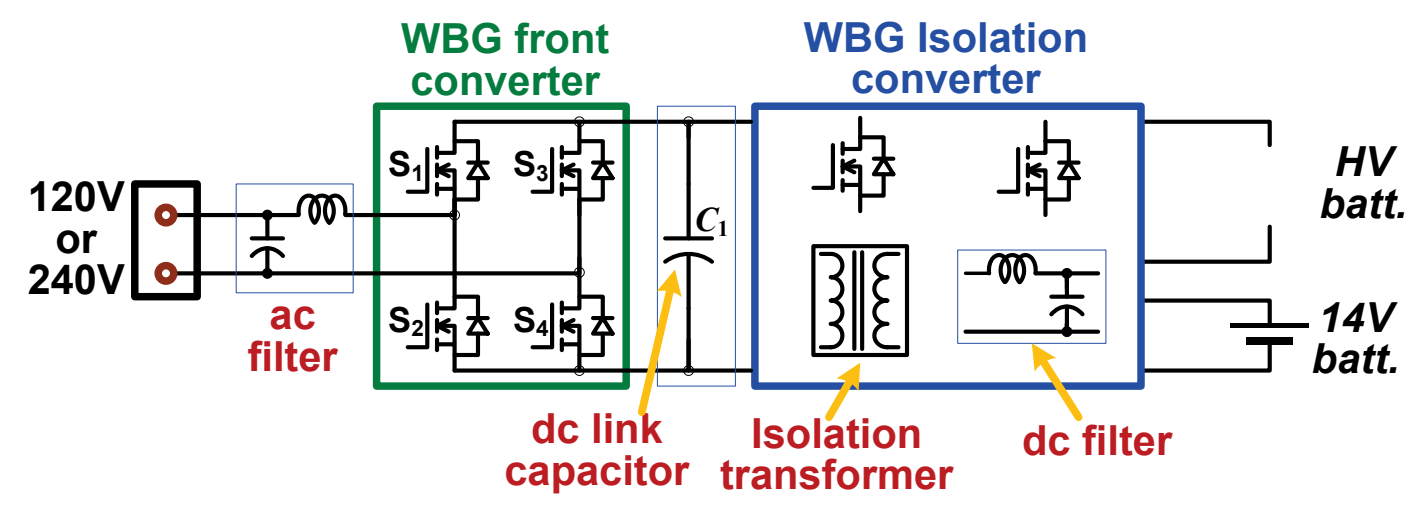

Figure 1: An integrated dc-dc converter and charger architecture.

Second, increasing power density and specific power without compromising efficiency is aggressively pursued by exploiting high switching frequency with WBG devices (especially GaN switches) and using advanced soft magnetic materials (nanocomposites) to drastically reduce the cost, weight, and volume of the ac and dc filters and isolation transformer. Because the availability of WBG power modules is limited, $\mathrm{SiC}$ and $\mathrm{GaN}$ devices are purchased or obtained directly from device vendors; they are tested, characterized, and packaged for use in converter design and prototype development. Prototypes will be built and tested first using SiC devices-for which wafer processing and device fabrication technologies have advanced to a stage such that SiC MOSFETs and other switches are available commercially - and then GaN switches as that technology matures and devices with high current ratings become available.

Third, further integration with traction drive systems is employed to reduce the component count for OBCs. For instance, WBG traction drive inverters and motors will be used to operate as the active front converter and to replace the ac filter inductor, significantly reducing $\mathrm{OBC}$ cost, weight, and volume.

Finally, a control strategy for the isolation converter has been developed to shrink the bulky de link capacitor. Without adequate control, this bulky capacitor is necessary to filter out the large voltage ripple - with twice the grid supply frequency-inherent in single-phase ac-dc converters. The proposed control strategy enables a $60 \%$ reduction in the ripple current and thereby a significant size reduction in the bulky dc link capacitor in the front ac-dc converter.

Figure 2 shows the integrated isolation converter topology selected through simulation. The converter is based on a phase-shifted dual active H- bridge converter that consists of two H-bridges (HB1 and HB2) and a buck converter coupled through a high-frequency transformer (Tr), which provides galvanic isolation for charging the batteries. One H-bridge is connected to the high-voltage traction battery and the other to the active front acdc converter. The H-bridge connected to the high-voltage battery, transformer, and buck converter forms a typical $14 \mathrm{~V}$ accessory converter, rated at around $2 \mathrm{~kW}$ in plug-in electric vehicles, for charging the $14 \mathrm{~V}$ battery and powering the vehicle's low-voltage accessory loads. Sharing the transformer and other switch components between the $\mathrm{OBC}$ and the $14 \mathrm{~V}$ converter leads to substantial cost, weight, and volume savings for the OBC compared with a stand-alone counterpart. Other features of the integrated charger include these: (1) It provides bidirectional power flow and thus can offer additional desired functions, such as vehicle-to-grid and vehicle-to-home applications. (2) It can charge the $14 \mathrm{~V}$ battery from the grid in addition to normal operation from the high-voltage traction battery. (3) It uses the parasitic capacitance of the switches and the transformer leakage inductance to achieve zero-voltage switching for EMI noise reduction and efficiency improvement. (4) The dual H-bridge converter enables the OBC to charge the battery over a wide range of voltages by providing a voltage buck-and-boost function through phase shifting and duty ratio control. 


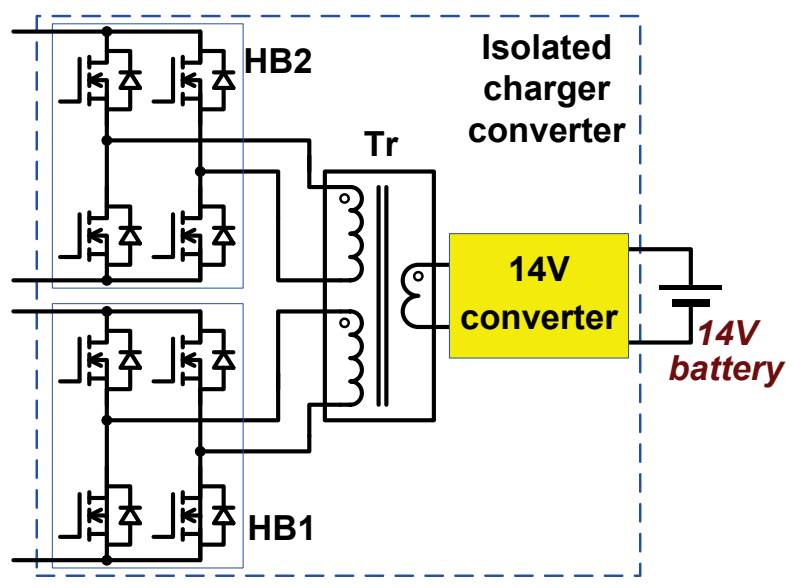

Figure 2: Dual active H-bridge-based isolated charger converter topology.

Figure 3 shows the block diagram of a segmented electrical drive system with the proposed integrated OBC functionality. It consists mainly of a high-voltage battery, a segmented traction drive system, three sets of contact switches (CS1, CS2 and CS3), and the isolated battery charging converter (Figure 2). The segmented traction drive system is realized by separating the switch dies of the inverter (INV) and the stator windings of the motor into two groups and connecting the groups of the switches and windings as two drive units. The segmented traction drive can significantly $(>60 \%)$ reduce the dc bus capacitor by performing interleaved switching between the two drive units. The neutral points of the two stator winding groups (N1 and N2) are brought out to a charging port through the contact switch, CS3. In addition, as in a stand-alone charger, a common mode and/or differential mode filter is usually used to filter out switching harmonics and common mode noises to meet power quality standards and safety regulations. The differential mode filter is typically realized with a capacitor and an inductor connected across and in series, respectively, with the charging port. One advantage of the integrated charger is that the motor is used as the filter inductor (as is described later), thus eliminating the need for an external filter inductor. The use of WBG switches further reduces the needed inductance. A $100 \mathrm{~kW}$ segmented inverter prototype using SiC MOSFETs was developed during FY 2015 for use in demonstrating an integrated $\mathrm{OBC}$ function. Details of the prototype are provided in the Results section.

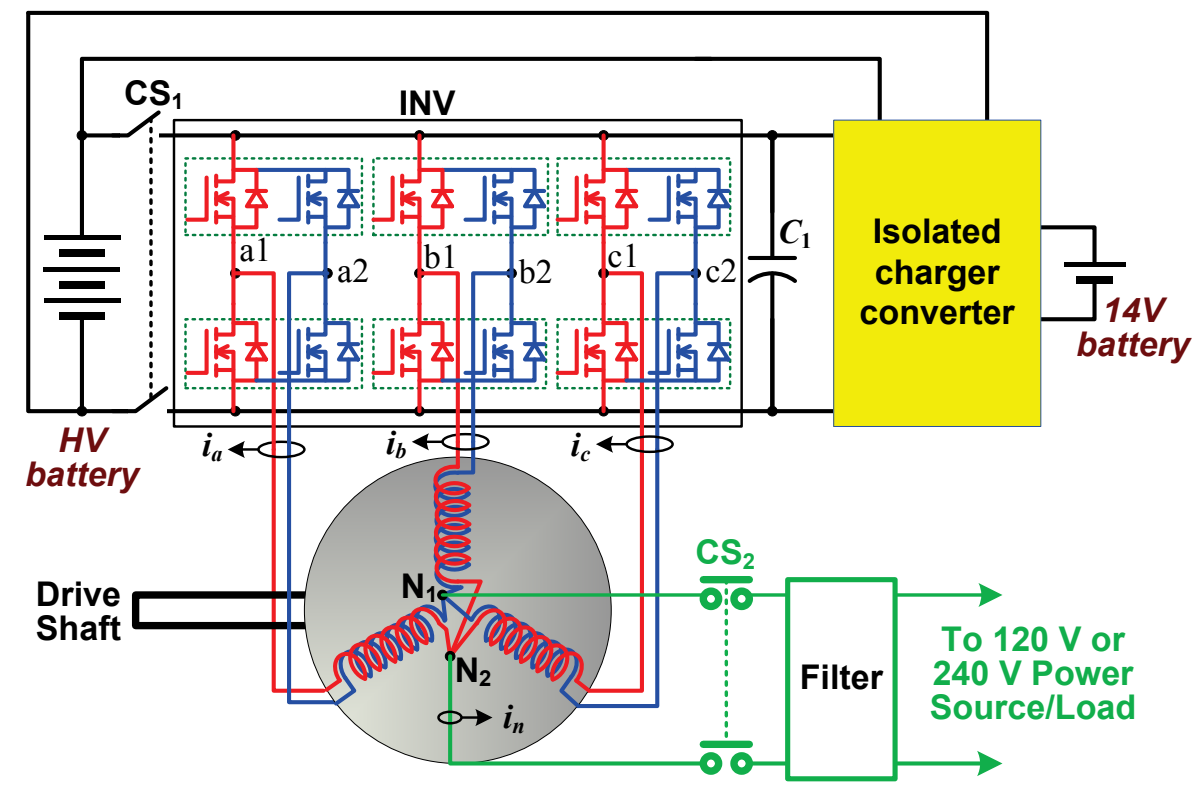

Figure 3: An example of the proposed integrated ОВC based on the segmented traction drive topology.

The electric drive system has three operation modes: (1) propulsion mode-providing the propulsive force for driving the vehicle and charging the $14 \mathrm{~V}$ battery, (2) charging mode - charging the high-voltage battery, and (3) sourcing mode - supplying power to external loads. 
In propulsion mode, contact switch CS1 is closed and CS2 is open, connecting the high-voltage battery to the drive units while disconnecting the charging converter and the charging port from the drive units. The two drive units operate with interleaved switching to reduce the dc bus ripple current, and thus the required size for the dc bus capacitor, and to control the speed and/or torque of the motor according to the amount of power required to propel the vehicle. Two (at minimum) or three current sensors that measure the combined currents of each respective phase are used in the motor control. In the meantime, the H-bridge and $14 \mathrm{~V}$ converter operate to charge the $14 \mathrm{~V}$ battery from the high-voltage battery.

In charging mode, contact switch CS1 is open and CS2 and CS3 are closed, disconnecting the high-voltage battery from the drive units while connecting the charging converter and the external source to the drive units. Figure 4 shows an equivalent circuit in this mode, where the capacitor is a filter component. All the switch legs in each of the INVs collectively function as a single switch leg, and the motor functions as a set of inductors. The latter is enabled by the motor's zero sequence (ZS) impedance network (ZSIN) consisting of three branches bundled together at the neutral point, with each branch formed by the motor leakage inductance and stator winding resistance ( $\operatorname{lm} 0 \mathrm{~s}$ and $\mathrm{rms}$ ). Together, the two drive units form a single-phase front active converter to regulate the dc bus voltage and perform power factor correction or reactive power control. A smaller current sensor is used to sense the grid current for use in the controller of the front converter. Moreover, the two H-bridges, HB1 and HB2, and the transformer operate as a zero-voltage switching phaseshifted dual-active-bridge converter to charge the high-voltage battery. If needed, the buck converter can also be activated to charge the $14 \mathrm{~V}$ battery. In this mode, the motor acts as a coupled inductor, and the resulting ZS current will not generate a rotating air-gap flux and thus will not produce any torque.

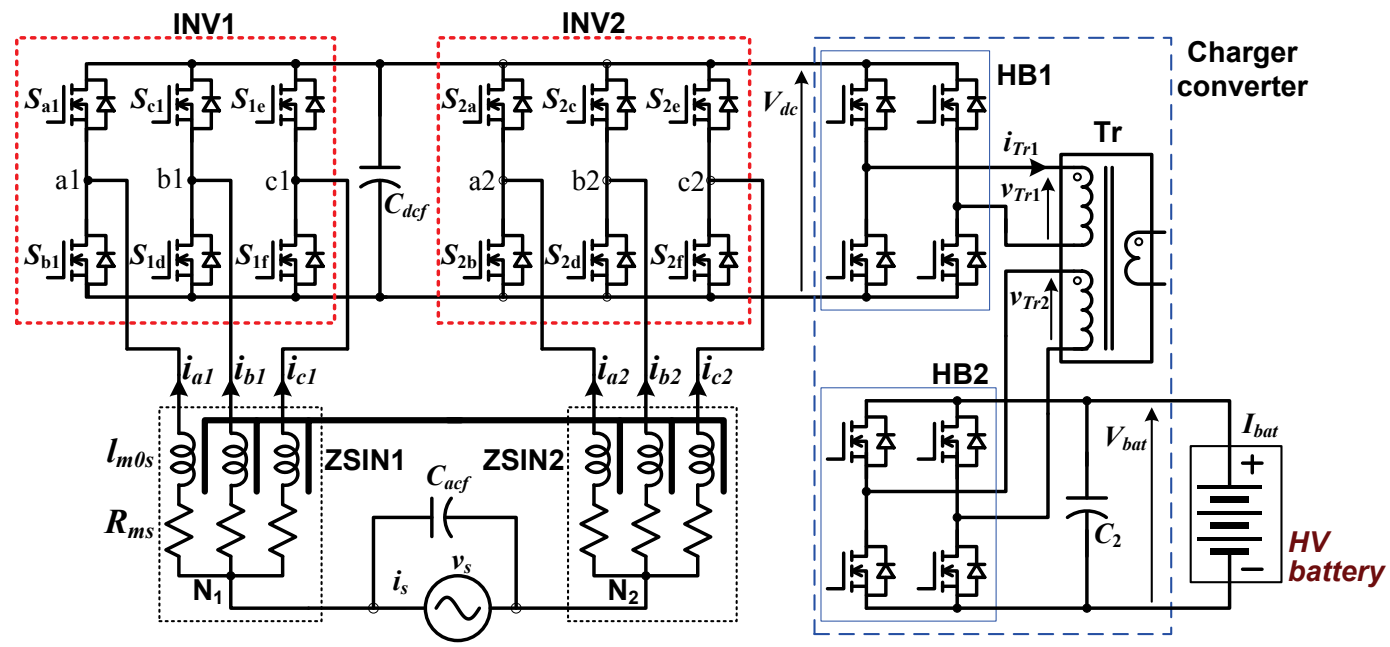

Figure 4: Equivalent circuit for operating in the charging mode.

During operation in the sourcing mode, the power flow is reversed from that in the charging mode. The two drive units form a single-phase inverter to supply either external loads or the grid. In this mode, the H-bridges operate to supply dc power from the high-voltage battery to the single-phase inverter, which in turn converts the dc power to ac power to the external load. If needed, the buck converter can be activated to charge the $14 \mathrm{~V}$ battery. Again as in the charging, the motor functions as a set of filter inductors provided by the ZS network. The grid current splits into three equal parts, and each part flows in each branch of the motor ZS network; therefore, the currents do not produce air-gap flux or generate any torque in the motor.

Figure 5 shows a control block diagram that consists of three control loops. The dc bus voltage control loop for maintaining a constant dc bus voltage at a commanded level of $\mathrm{V}_{\mathrm{dc}}^{*}$ is implemented with a proportional integral (PI) regulator, which generates a portion of the amplitude $\left(\mathrm{I}_{\mathrm{s}}^{*}\right)$ of the current command, $\mathrm{i}_{\mathrm{s}}^{*}$, for the inner grid current control loop. The other part of the amplitude of the current command is provided by a feed forward compensation determined by the battery charging power command, $\mathrm{P}_{\text {bat }}^{*}$, modified by a feed-forward gain, kff. The battery charging power command is generated in the charger converter controller and is described below. The current regulator (GI), whose purpose is to produce a near sinusoidal grid current with unity power factor (or a commanded value for reactive power compensation), can be implemented with a simple gain block of a relatively high value or a PI regulator to generate pulse-width-modulated gating signals for the two INVs. As 
shown in the figure, low pass filters (LPFs) are used in the feedback paths to remove the high-frequency components in the sensed voltages and currents.

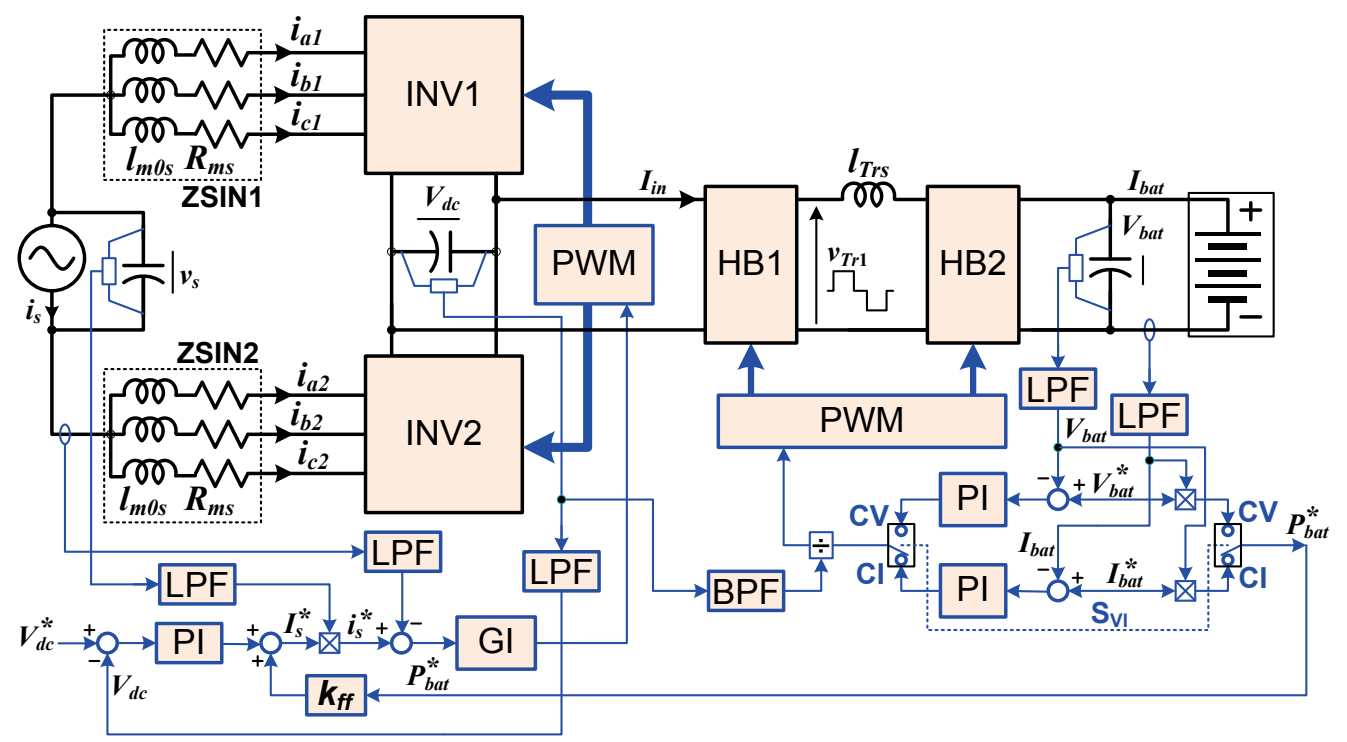

Figure 5: Control block diagram.

The third control loop is for the charger converters, HB1 and HB2. The purpose of this control is to maintain a constant battery terminal voltage at a commanded level of $\mathrm{V}_{\text {bat }}^{*}$ in the constant voltage $(\mathrm{CV})$ charging mode or a constant current $(\mathrm{CI})$ at a commanded level of $\mathrm{I}_{\text {bat }}^{*}$ in the CI charging mode. A software switch, SVI, is used to select the charging mode, according to the state of charge of the battery. Again, a PI controller is used to regulate the battery voltage or current. Because of the significantly smaller dc bus capacitor in the segmented inverter, the dc bus voltage, $\mathrm{Vdc}$, will fluctuate to a greater degree during operation in single-phase charger mode. To prevent it from causing a large ripple component in the battery charging current, the PI output is divided by the high-frequency component of Vdc, obtained with a band-pass filter and then fed to the pulsewidth-modulation block, which controls the duty cycles. It also regulates the phase shift between the two H-bridges, if needed.

In addition, the battery charging power command, $\mathrm{P}_{\mathrm{bat}}^{*}$, is computed by

$$
P_{b a t}^{*}=\left\{\begin{array}{l}
V_{b a t}^{*} I_{b a t}, \text { in CV charging mode } \\
I_{b a t}^{*} V_{b a t}, \text { in CI charging mode }
\end{array}\right.
$$

where Vbat and Ibat are the measured battery terminal voltage and current. As mentioned, $P_{b a t}^{*}$ is used in the feed-forward compensation in the grid current control loop to improve the dynamic response of the dc bus voltage loop.

\section{Results and Discussion}

A prototype consisting of an electrical drive system and a charger converter was built to test the integrated charging functionality. A traction drive inverter was designed and built using six commercial SiC MOSFET phase-leg modules rated at $1,200 \mathrm{~V} / 120 \mathrm{~A}$, four film dc bus capacitors with a total $880 \mu \mathrm{F}$ of capacitance, a water-cooled cold plate $(36 \times 12.7 \mathrm{~cm})$, a 3D-printed mounting frame, and other fixture components. The $\mathrm{SiC}$ MOSFET modules contain antiparallel SiC Schottky barrier diodes, so the MOSFET body diodes with inferior characteristics are bypassed. The inverter design is flexible so that it can be configured as dual 3-phase inverters, each rated at $50 \mathrm{~kW}$, or a segmented 3-phase inverter, rated at $100 \mathrm{~kW}$, by swapping the output bus bar and current sensor assembly, as shown in Figure 6. Figure 7 shows a photo of the inverter prototype configured as a $100 \mathrm{~kW}$ segmented inverter used in the OBC tests. 


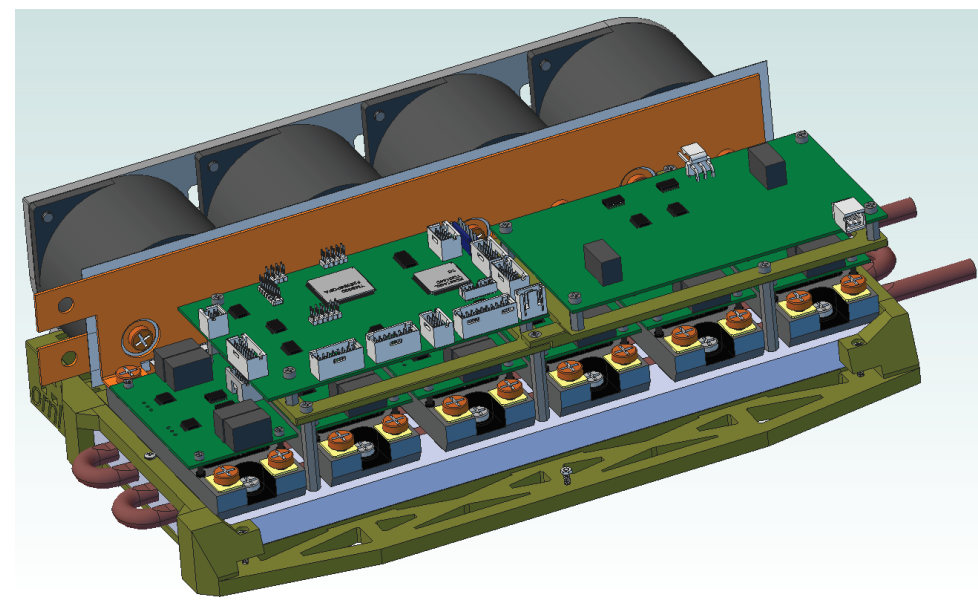

(a)

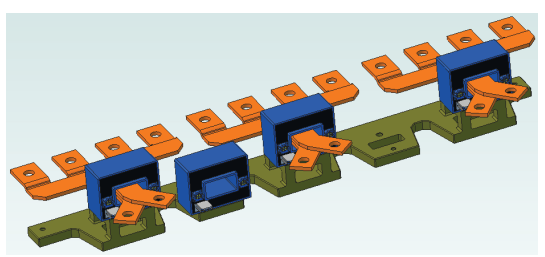

(b)

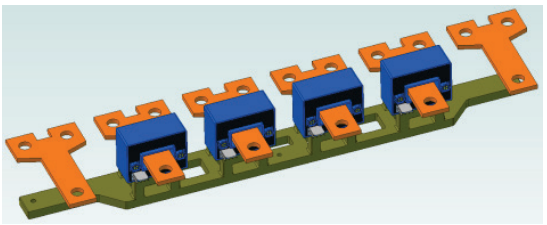

(c)

Figure 6: Design of an SiC traction drive inverter that can be operated as dual 3-phase inverters, each rated at $50 \mathrm{~kW}$, or a segmented 3-phase inverter, rated at $100 \mathrm{~kW}$, by swapping the output bus bar and current sensor assembly. (a) Inverter base assembly, (b) output bus bar and current sensor assembly for segmented inverter, (c) output bus bar and current sensor assembly for dual 3-phase inverters.

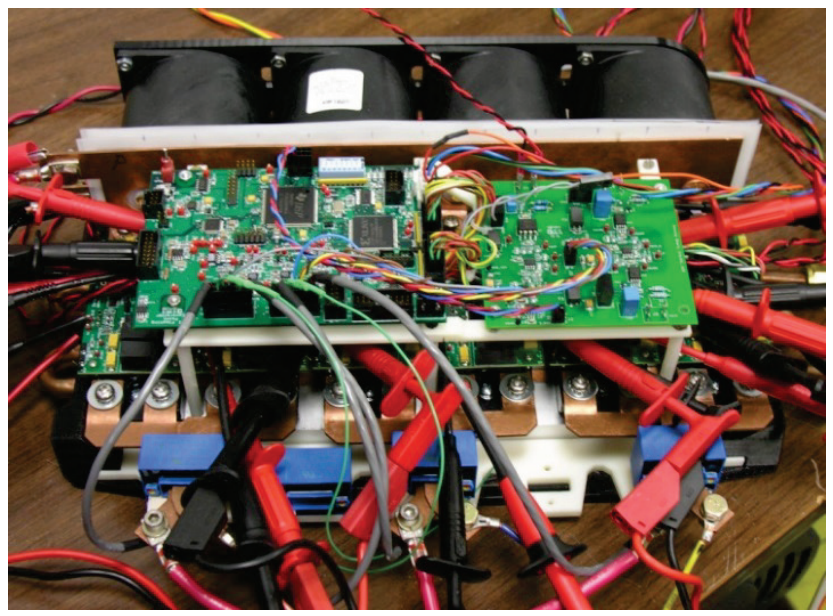

Figure 7: Photo of a $100 \mathrm{~kW} \mathrm{SiC} \mathrm{traction} \mathrm{drive} \mathrm{inverter} \mathrm{prototype} \mathrm{used} \mathrm{in} \mathrm{the} \mathrm{integrated} \mathrm{charger} \mathrm{tests.}$

A $6.8 \mathrm{~kW}$ charger converter prototype (Figure 8) was designed and built using four SiC phase leg modules, a planar transformer, and heavy copper PCBs to eliminate wire connections. Figure 8 also shows a photo of the $\mathrm{SiC}$ phase leg modules, which were designed and packaged in-house using direct-bonded copper substrates, Cree SiC MOSFETs, and Schottky barrier diodes. A control PCB using a TI TMS320F2809 fixed-point microcontroller with micro-edge-positioning-based high-resolution pulse width/phase shift capability (180 ps vs. $10 \mathrm{~ns}$ for normal resolution) was used to implement the battery charging voltage and current control blocks.

The charger converter was tested at switch frequencies of fsw $=100 \mathrm{kHz}, 200 \mathrm{kHz}$, and $250 \mathrm{kHz}$. Figure 9 shows typical operating waveforms at $\mathrm{fsw}=200 \mathrm{kHz}$ and $250 \mathrm{kHz}$. Figure 10 plots measured charger converter efficiencies. As expected, the efficiencies drop as the switching frequency increases. Maximum efficiency numbers are $99.0 \%$ at $100 \mathrm{kHz}, 97.5 \%$ at $200 \mathrm{kHz}$, and $97.3 \%$ at $250 \mathrm{kHz}$. 

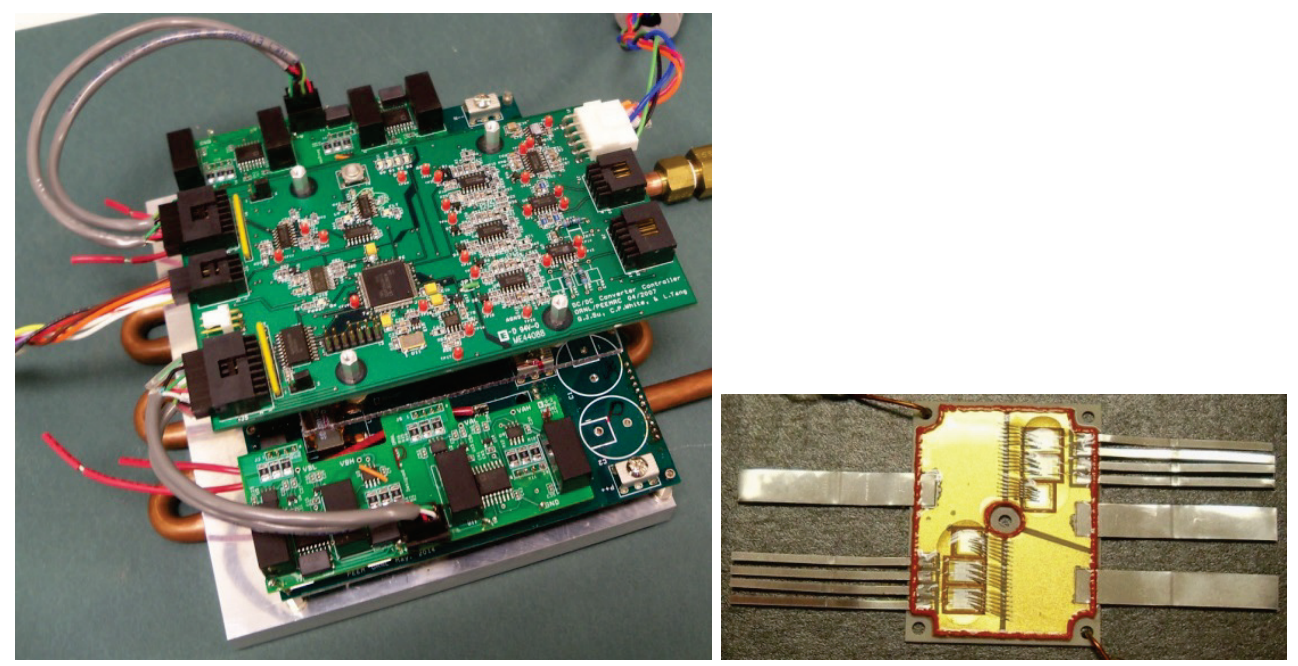

Figure 8: Photos of a $6.8 \mathrm{~kW}$ charger converter and SiC MOSFET phase-leg modules ( $36 \times 46 \mathrm{~mm})$.

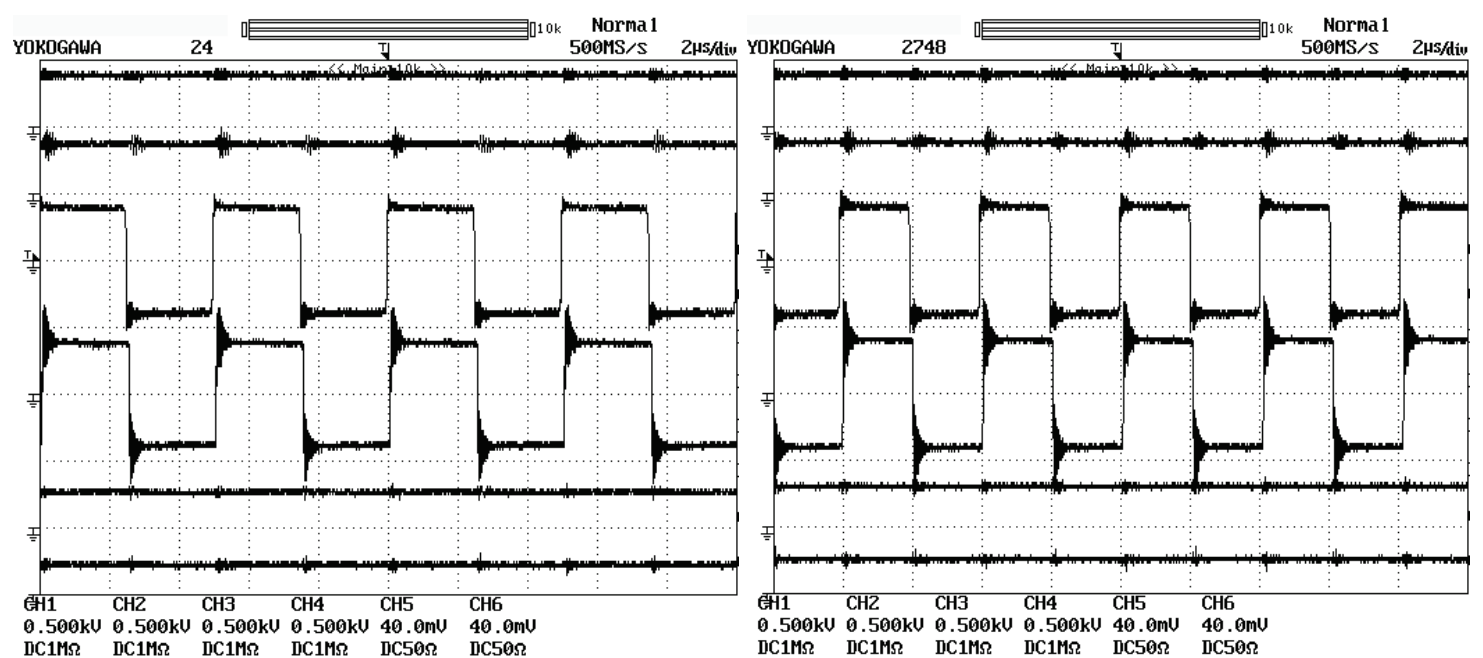

Figure 9: Typical operating waveforms of the SiC charger converter at fsw=200 kHz (left) and $250 \mathrm{kHz}$ (right). From top: input dc voltage (Vin, $500 \mathrm{~V} /$ div), output dc voltage (Vout, $500 \mathrm{~V} /$ div), transformer primary terminal voltage (vTr1, $500 \mathrm{~V} /$ div), transformer secondary terminal voltage (vTr2, $500 \mathrm{~V} /$ div), input current (lin, $40 \mathrm{~A} / \mathrm{div}$ ) and output current (lout, $40 \mathrm{~A} / \mathrm{div})$.

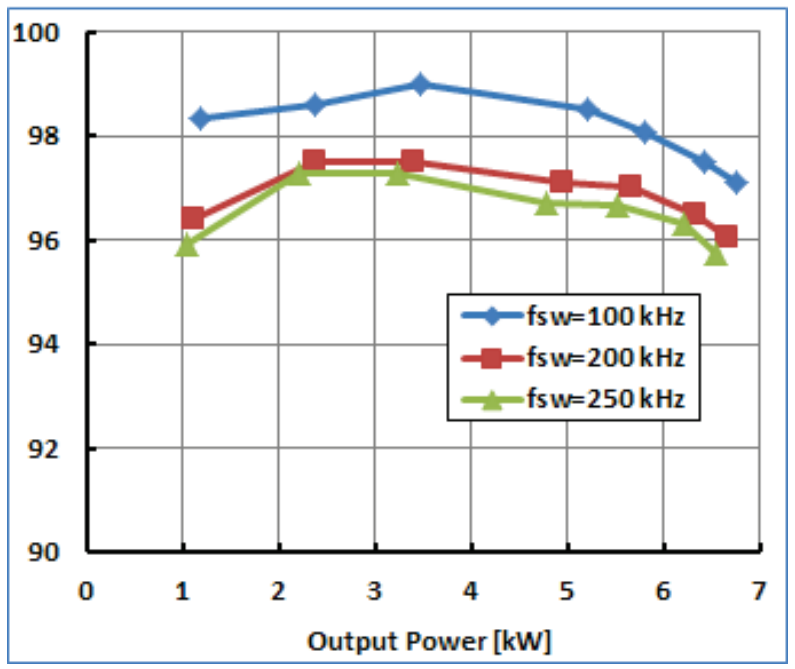

Figure 10: Measured charger converter efficiencies. 
The $\mathrm{SiC}$ segmented inverter and charger converter were then integrated together and connected to a commercial off-the-shelf induction motor to form an integrated $\mathrm{OBC}$ for performance tests. Figure 11 shows a photo of the test setup. The motor — rated at $14.9 \mathrm{~kW}, 230 \mathrm{Vrms}, 45.4 \mathrm{Arms}$ - has two poles and two sets of stator windings with all leads accessible.

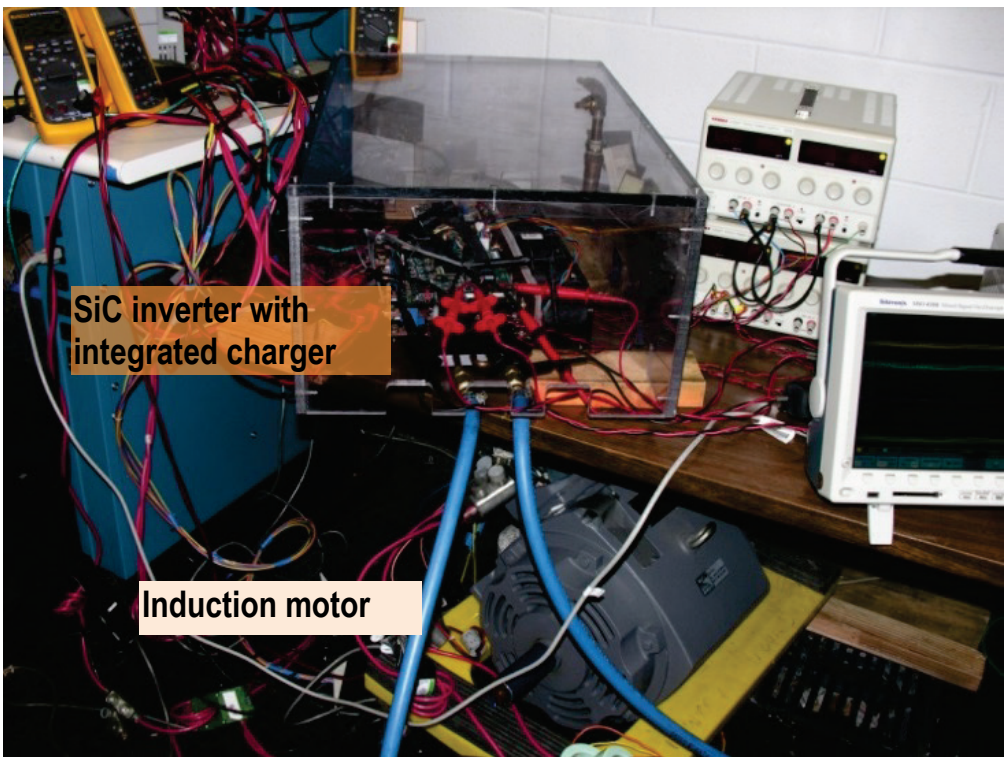

Figure 11: Photo of the test setup using the SiC segmented inverter, the charger converter, and a motor for an integrated $6.8 \mathrm{~kW} \mathrm{OBC.}$

The integrated OBC was tested successfully with a resistive load bank at both 120 and $240 \mathrm{~V}$ grid voltages. Representative waveforms were included to illustrate the operation of the integrated charger. Figure 12 shows typical operating waveforms of the system with a $120 \mathrm{~V}$ input voltage and charging power of $1.0 \mathrm{~kW}$ (left) and $1.7 \mathrm{~kW}$ (right). Figure 13 shows waveforms of the system with a $240 \mathrm{~V}$ grid voltage and charging power of $2.7 \mathrm{~kW}$ (left) and $6.8 \mathrm{~kW}$ (right). Figure 14 plots OBC system efficiencies. The maximum efficiency is $96.5 \%$ at a grid voltage of $240 \mathrm{~V}$ and $92.6 \%$ at $120 \mathrm{~V}$. A $2 \%$ point improvement over a silicon-based counterpart developed in FY 2013 was observed.
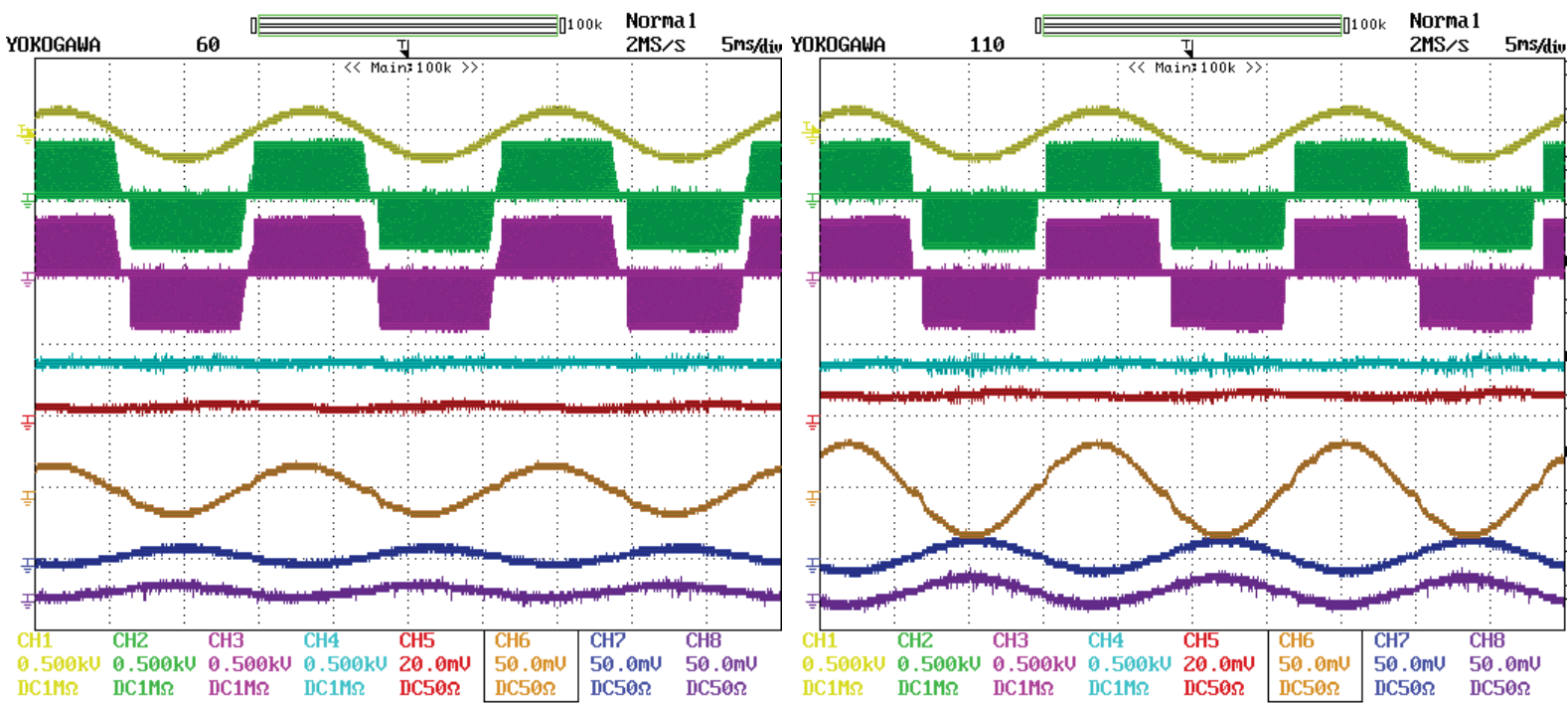

Figure 12: Waveforms of the OBC with $120 \mathrm{~V}$ input and $1.0 \mathrm{~kW}$ (left), $1.7 \mathrm{~kW}$ (right) charging power. From top: grid voltage (vs, $500 \mathrm{~V} /$ div, CH1), converter input voltages (va1a2, CH2, vb1b2, CH3, $500 \mathrm{~V} /$ div), dc bus voltage (Vdc, $500 \mathrm{~V} / \mathrm{div}, \mathrm{CH} 4)$, charging current (Ibat, $20 \mathrm{~A} /$ div, CH5), grid current (is, $50 \mathrm{~A} / \mathrm{div}, \mathrm{CH} 6$ ) and motor phase a1 and a2 currents (ia1, CH7, ia2, CH8, $50 \mathrm{~A} /$ div). 


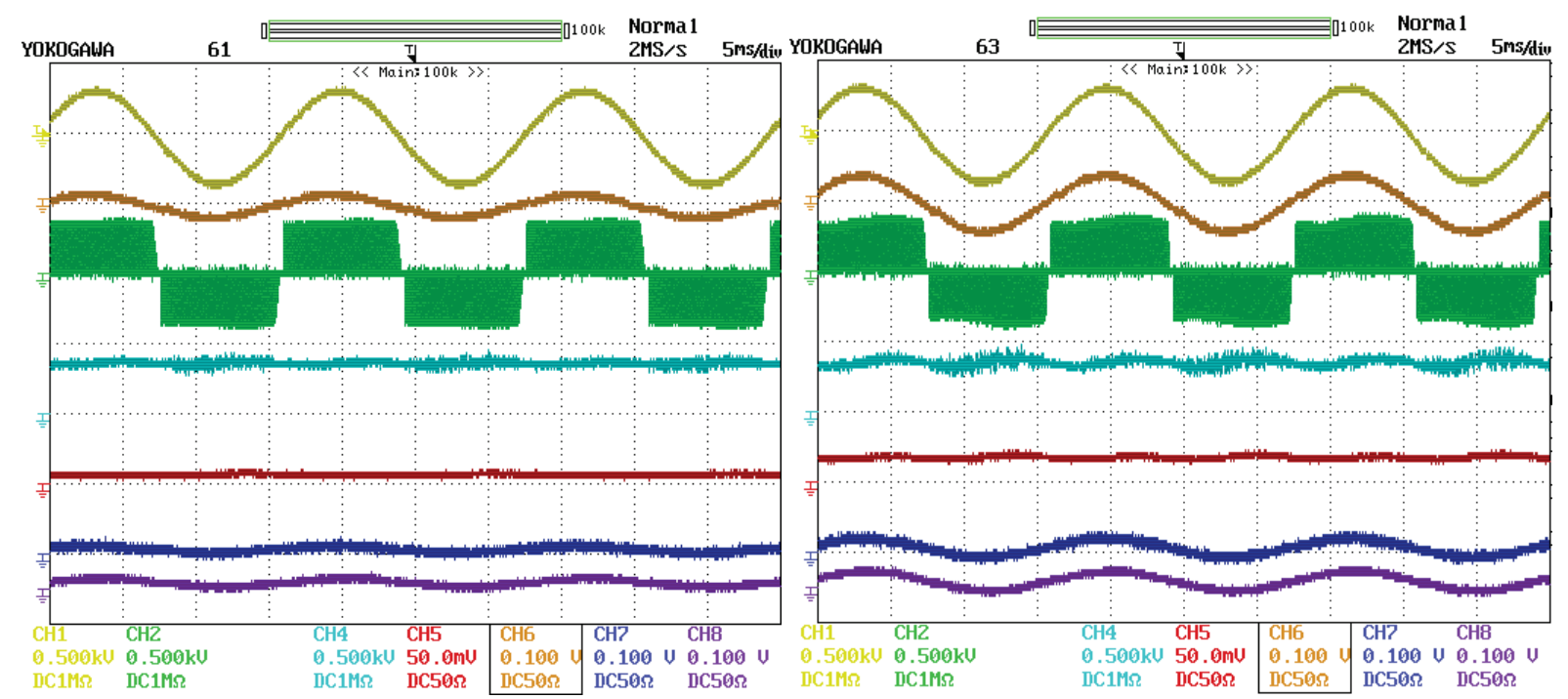

Figure 13: Waveforms of the $\mathrm{OBC}$ at $240 \mathrm{~V}$ input and $2.7 \mathrm{~kW}$ (left), $6.8 \mathrm{~kW}$ (right) charging power. From the top: grid voltage (vs, $500 \mathrm{~V} / \mathrm{div}, \mathrm{CH} 1$ ), grid current (is, $100 \mathrm{~A} / \mathrm{div}, \mathrm{CH} 6)$, converter input voltage (va1b1, $500 \mathrm{~V} / \mathrm{div}, \mathrm{CH2}$ ), dc bus voltage (Vdc, $500 \mathrm{~V} /$ div, CH4), charging current (lbat, $50 \mathrm{~A} / \mathrm{div}, \mathrm{CH} 5$ ) and motor phase a1 and a2 currents (ia1, CH7, ia2, CH8, 100 A/div).

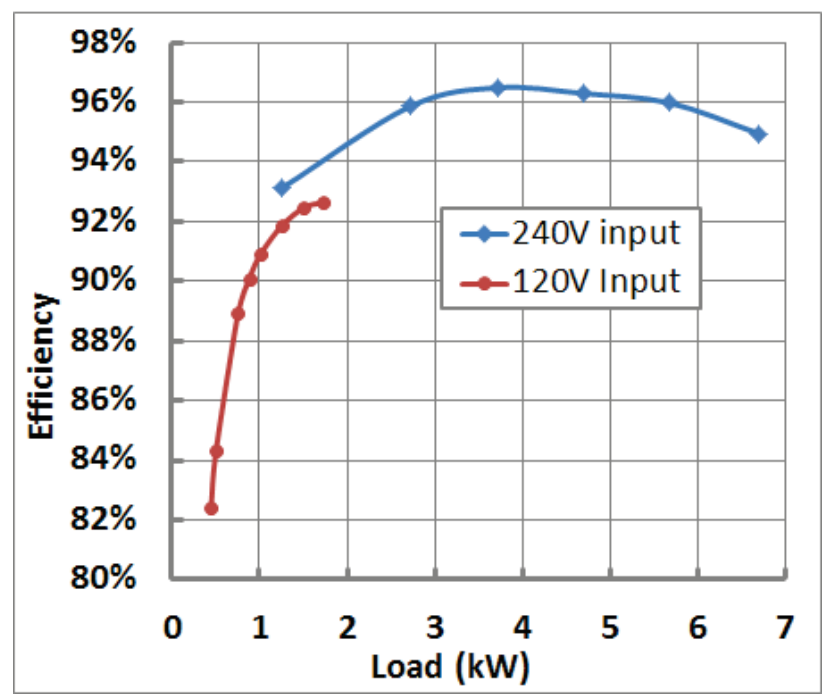

Figure 14: Measured OBC system efficiencies at grid voltages of 120 and $240 \mathrm{~V}$.

In collaboration with Aegis Technology, an advanced magnetic material-based core set of an E-shaped core and a plate (Figure 15) was made at ORNL's Manufacturing Demonstration Facility using a nanocomposite magnetic powder supplied by Aegis Technology. The powder is a mixture of magnetic nanoalloy (FeNbSiCuB) particles (60 vol \% or $90 \mathrm{wt} \%$ ) and polyphenylene sulfide (PPS) polymer powders (40 vol \% or $10 \mathrm{wt} \%$ ). The cores were printed with an inkjet 3D printer using the powder, and then baked in an oven at $320^{\circ} \mathrm{C}$ for a little over an hour to set the PPS polymer in the mixture. The cores produced were a PPS-bound magnetic nanocomposite with an operating temperature of up to $200^{\circ} \mathrm{C}$. The core set was designed to emulate a commercial-off-the-shelf ferrite core and is being comparatively evaluated against the commercial product for high-frequency inductor and transformer applications. 


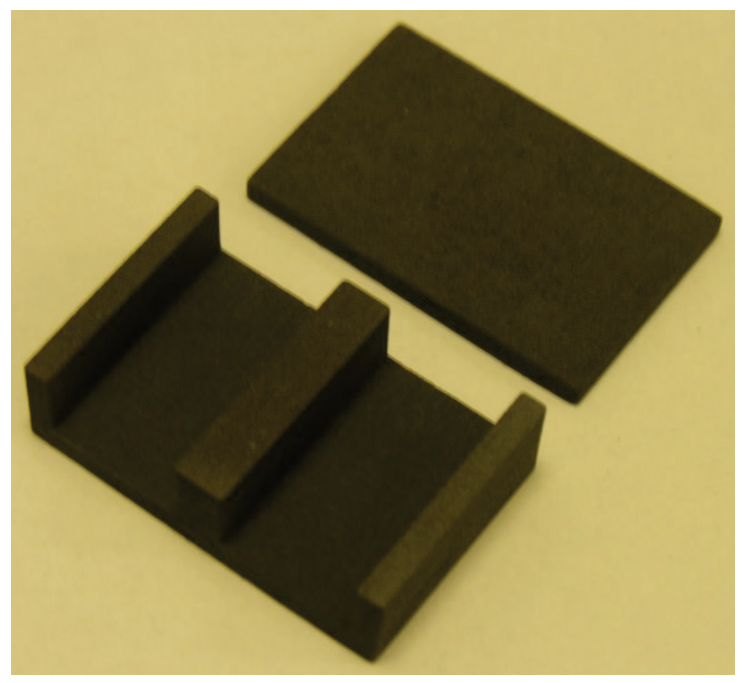

Figure 15: Photo of the 3D-printed nanocomposite magnetic E and I cores $(58 \times 38 \times 17 \mathrm{~mm})$.

A $6.8 \mathrm{~kW}$ charger converter was designed using GaN Systems GaN transistor GS66516T. The GaN transistor is rated at $650 \mathrm{~V}$ and $47 \mathrm{~A}$ continuous power at a case temperature of $100^{\circ} \mathrm{C}$. The design involves customization of a liquid-cooled cold plate; PCB design for the high-voltage heavy copper power planes for mounting the GaN transistors, dc bus capacitors, and transformer windings; PCB design for the $14 \mathrm{~V}$ highcurrent power plane for mounting the $14 \mathrm{~V}$ transformer winding and buck converter; planar transformer core design; gate drive PCBs; and a digital signal processing control PCB. Figure 16 shows the converter design (left) and the assembly of the power planes and a planar transformer (right). The dimensions of the converter are $7 \times 4.75 \times 1.75 \mathrm{in}$., giving a power density of $7.1 \mathrm{~kW} / \mathrm{L}$. A purchasing order for the $\mathrm{GaN}$ transistors has been issued. Once the devices are received, a prototype will be built and tested in FY 2016.
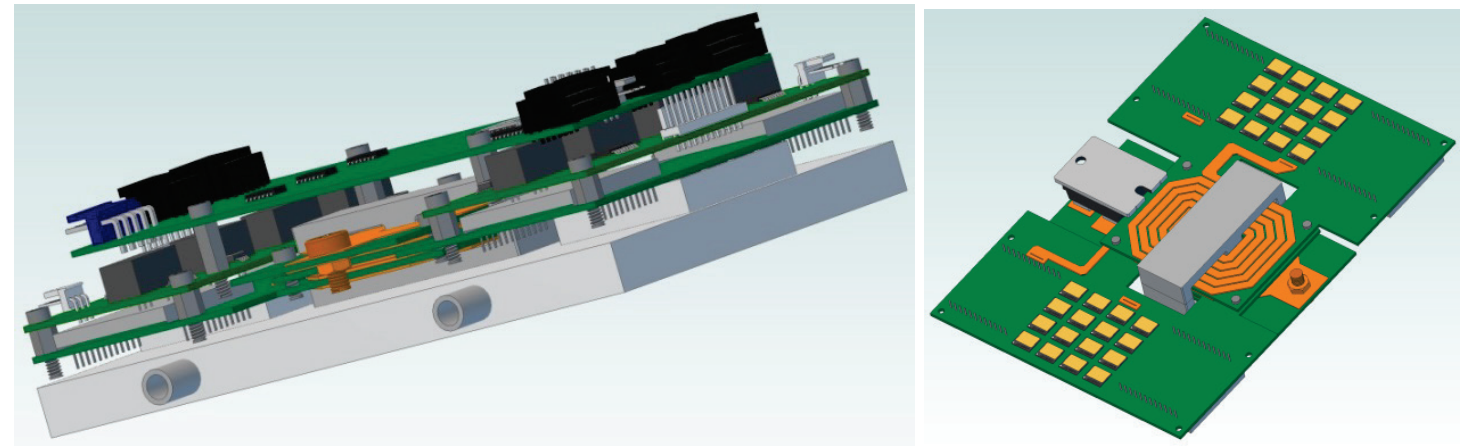

Figure 16: Design for a $6.8 \mathrm{~kW}$ charger converter using a GaN transistor (left) and power plane and planar transformer assembly (right).

\section{Conclusions and Future Direction}

This project is aimed at leapfrogging existing silicon-based charger technology to address charger and converter cost, weight, volume, and efficiency. It proposes to overcome the limitations of silicon semiconductor and magnetic materials by using WBG devices, including those made with $\mathrm{SiC}$ and GaN; using advanced magnetic materials; and employing a novel integrated charger architecture and control strategy.

Under this multiyear project, a new integrated $\mathrm{OBC}$ and dc-dc converter architecture has been developed that integrates the segmented traction drive, $14 \mathrm{~V}$ dc-dc converter, and a high-voltage battery charger dc-dc converter. The new topology significantly reduces the number of components: it achieves a $47 \%$ reduction in power circuit components alone, not counting savings in the gate driver and control logic circuits, translating to a $50 \%$ reduction in cost and volume compared with existing stand-alone OBCs. In addition, WBG-based devices are employed in the converter and inverter to further reduce the cost, weight, and volume of the 
passive components, as well as improve system efficiency. A control strategy for the charger isolation converter was also developed to reduce the battery ripple current inherent in single-phase ac-dc converters.

The control strategy was shown to reduce the ripple current by $60 \%$, enabling a corresponding reduction in the bulky dc link capacitor in the active front end converter.

A $6.8 \mathrm{~kW}$ SiC-based OBC prototype based on the new topology was designed, built, and tested by integrating a 3-port isolated $\mathrm{SiC}$ dc-dc converter with a $100 \mathrm{~kW} \mathrm{SiC} \mathrm{traction} \mathrm{inverter.} \mathrm{Test} \mathrm{results} \mathrm{showed} \mathrm{it} \mathrm{to} \mathrm{have} \mathrm{a} \mathrm{peak}$ efficiency of $96.5 \%$ when charged from a $240 \mathrm{~V}$ source and of $92.6 \%$ when charged from a $120 \mathrm{~V}$ source.

The test results also show a $2 \%$ point improvement over the silicon-based counterpart.

Progress was made on employing GaN switches and advanced nanocomposite magnetic materials for OBCs. A design for a $6.8 \mathrm{~kW}$ charger converter was completed using GaN Systems GaN transistors, high-voltage heavy copper PCB power planes, low-voltage $(14 \mathrm{~V})$ high-current PCB power planes, and a planar transformer. The design has a high power density of $7.1 \mathrm{~kW} / \mathrm{L}$. In addition, in collaboration with Aegis Technology, an advanced magnetic material core set of an E-shaped core and a plate was fabricated using ORNL's additive manufacturing capability and Aegis Technology's nanocomposite magnetic powders.

Future work will be directed at designing, building, and testing prototypes for a $6.6 \mathrm{~kW} \mathrm{GaN}$ isolation converter and $\mathrm{OBC}$ and a $2 \mathrm{~kW}$ GaN $14 \mathrm{~V}$ converter.

\section{FY 2015 Presentations/Publications/Patents}

1. G. J. Su and L. Tang, "An integrated onboard charger and accessory power converter using WBG devices," in Proceedings of the 7th IEEE Energy Conversion Congress and Exposition (ECCE 2015), pp. 6306-6313, Montreal, Canada, September 20-24, 2015.

2. G. J. Su, "Innovative technologies for converters and chargers," presented at the DOE Vehicle Technologies Office Electric Drive Technologies Advanced Power Electronics and Electric Motors R\&D FY 2015 Kickoff Meeting, Oak Ridge, Tennessee, November 18-20, 2014.

3. G. J. Su, "Innovative technologies for converters and chargers," presented at the 2015 DOE Hydrogen and Fuel Cells Program and Vehicle Technologies Office Annual Merit Review and Peer Evaluation Meeting, Arlington, Virginia, June 8-12, 2015. 


\subsection{Traction Drive Systems with Integrated Wireless Charging}

\section{Gui-Jia Su, Principal Investigator}

Oak Ridge National Laboratory (ORNL)

National Transportation Research Center

2360 Cherahala Boulevard

Knoxville, TN 37932

Phone: (865) 946-1330

E-mail: sugj@ornl.gov

\section{Susan A. Rogers, DOE EDT Program Manager}

Phone: (202) 586-8997

E-mail: Susan.Rogers@ee.doe.gov

\section{Burak Ozpineci, ORNL EDT Program Manager}

Phone: (865) 946-1329

E-mail: burak@ornl.gov

Contractor: UT-Battelle, LLC, managing and operating contractor for the Oak Ridge National Laboratory Contract No.: DE-AC05-00OR22725

\section{Abstract/Executive Summary}

- The project aims to redesign the electric drive system for plug-in electric vehicles to include wireless charging functionality and reduce cost while increasing efficiency and power density using wide bandgap (WBG) devices.

- The FY 2015 objective is to develop converter topologies suitable for traction drive systems with integral wireless charging functionality and control strategies for minimizing component size and circuit losses through detailed circuit simulation.

- An optimal resonant circuit was designed that can significantly reduce the resonant circuit current and the losses.

- Three electric drive topologies were developed and proved to be functional by circuit simulation.

\section{Accomplishments}

- Simulated various resonant circuits and designed one that is optimized for minimizing circulating current and the associated losses. It shows, with the optimized resonant circuit, a range of $61-75 \%$ reduction in the primary current and $10-44 \%$ reduction in the total losses in a dc-ac wireless charger.

- Simulated and proved concepts for three electric drive topology candidates with integrated wireless charging functionality:

- Topology 1: Tapping into the $14 \mathrm{~V}$ accessory power supply converter to eliminate the secondary ac-dc converter for the wireless charger.

- Topology 2: Using the traction motor and inverter to eliminate the secondary ac-dc converter for the wireless charger

- Topology 3: Using a multiport dc-dc converter that combines a reduced-power boost converter for stepping up the dc bus voltage of the traction drive inverter, a $14 \mathrm{~V}$ buck converter for powering the $14 \mathrm{~V}$ vehicle accessory loads, and a wireless battery charging converter.

- Simulation results show all the wireless chargers have high input power factors of greater than $99 \%$ and low total harmonic distortion (THD) factors of less than $3.5 \%$ in the ac source current. 


\section{Introduction}

Wireless power transfer is emerging as a safe, convenient charging technology for electric and plug-in hybrid electric vehicles (EVs and PEVs). With minimal or no user intervention and no need for a cable and plug (the vehicle need only be parked at a specified location), wireless chargers offer ultimate convenience. Automated charging using sensors and wireless communication systems can maximize the electrically powered mileage because it eliminates the problem of users forgetting to plug in their vehicles.

The wireless power transfer technology used by most systems on the market today is inductive power transfer, which uses a fair amount of magnetic core material to enhance the flux coupling effect. Figure 1 illustrates a conceptual block diagram for wireless chargers based on two loosely coupled coils in which a $50 / 60 \mathrm{~Hz}$ ac voltage is converted by a power factor correction (PFC) converter and a high-frequency inverter into a highfrequency voltage in the range from tens of kilohertz to a few megahertz. The ac voltage is transmitted to an onboard ac-dc converter through the loosely coupled coils and converted to a dc voltage level suitable for charging the battery. As illustrated in Figure 1, a wireless charger is divided into off-vehicle components (positioned in garages or other charging stations) and in-vehicle components; thus, compared with an onboard wired charger, it reduces the cost to the vehicle of charging the battery.

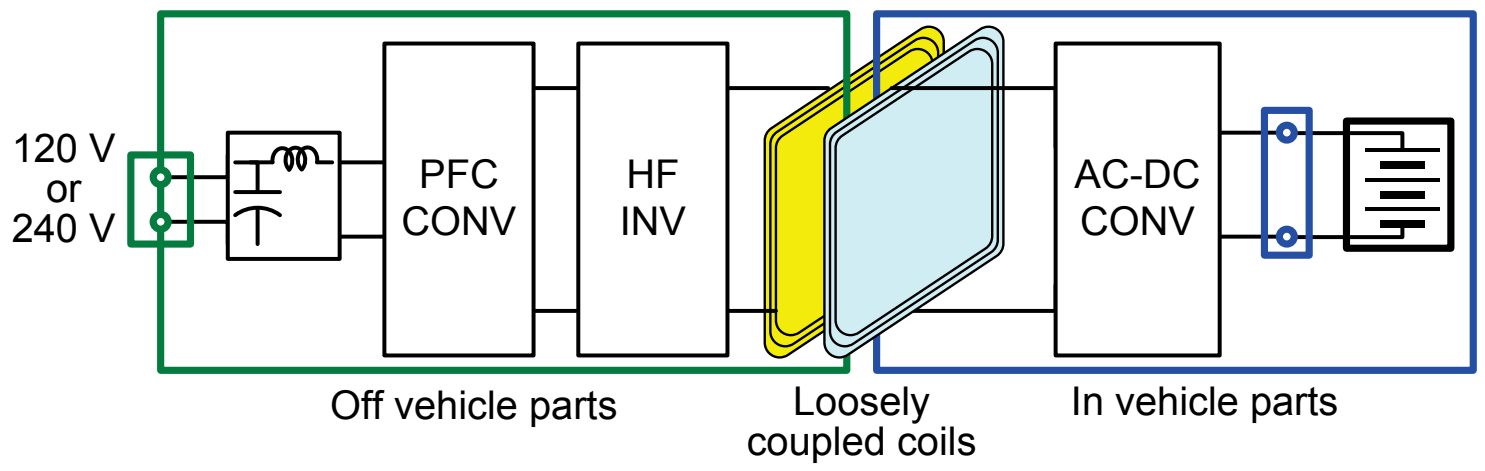

Figure 1: A conceptual block diagram for wireless chargers based on loosely coupled coils.

Because typical wireless chargers employ a PFC front-end converter, a high-frequency inverter, a resonant circuit including a set of coupled transmitting and receiving coils, and an ac-dc converter, they require a large number of semiconductor devices and passive components. These lead to high cost, weight, and volume, which restricts wireless chargers from mass production. The performance of ac-dc and dc-ac converters is further constrained by the limitations of current semiconductor and magnetic materials: (1) silicon switches restrict switching frequencies to typically $100 \mathrm{kHz}$, and (2) soft ferrite magnetic material-based inductors and transformers further limit power density and efficiency because of their low saturation flux densities and high core losses at high frequencies. As a result, passive components contribute significantly to the cost, weight, and volume of wireless charging systems. In addition, efficiency is low, less than $90 \%$.

Moreover, existing EVs and PEVs employ individually optimized converters for the electric traction drive and the battery charger, leading to high component counts, system weight, volume, and cost. The objective of this project is therefore to redesign the electric drive system with integral wireless charging functionality to minimize electric drive system cost, weight, and volume and maximize efficiency.

The project takes advantage of WBG semiconductors, including $\mathrm{SiC}$ and gallium nitride (GaN), and advanced magnetic materials to enable a substantial reduction in the cost, weight, and volume of passive components and an increase in efficiency. WBG semiconductors permit devices to operate at much higher temperatures and frequencies with lower losses. And advanced soft magnetic materials allow transformers and inductors to shrink significantly in volume, weight, and core loss. Together, these semiconductor and magnetic materials can make ac-dc and dc-dc converters and inverters significantly more compact and energy-efficient than those made from conventional materials. 


\section{Approach}

Our approach will focus on (1) minimizing PEV system components and cost through functional integration and (2) increasing efficiency. Our goal for wireless charging efficiency is greater than $92 \%$.

Figure 2 shows a conceptual block diagram for a traction drive system with integral wireless charging functionality. Our strategies to address the issues of existing systems are (1) integrate the onboard portion of a wireless charger into the traction drive power electronics system to reduce the number of components; (2) use WBG devices made of materials including $\mathrm{SiC}$ and $\mathrm{GaN}$ and advanced magnetic materials to reduce the cost, weight, and volume of passive components; (3) develop control strategies to minimize the resonant current and voltage to shrink the cost and size of the resonant components and their losses; and (4) develop coil designs for maximum coupling coefficient and minimum fringe field level through novel geometries and optimization of coils and ferrite shields.

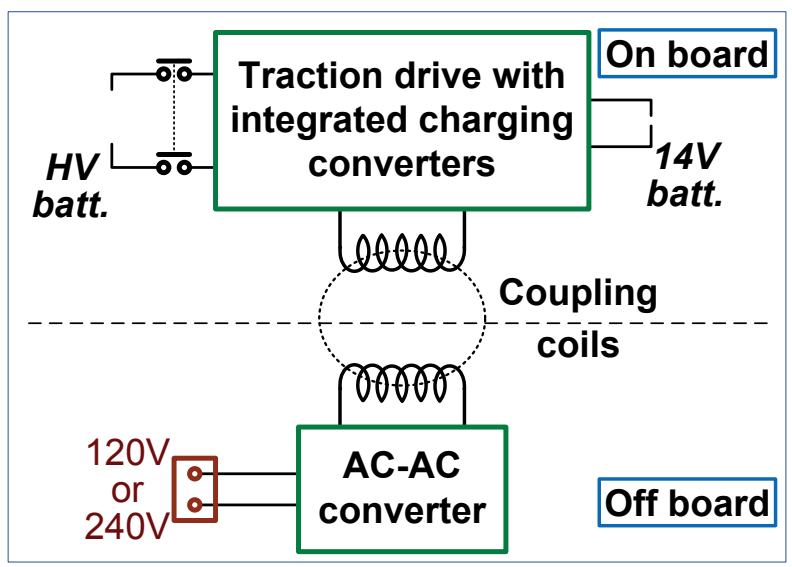

Figure 2: Conceptual block diagram for a traction drive system with integral wireless charging functionality, in which the traction drive inverters and motors are used as part of the onboard ac-dc converter to eliminate or minimize the number of components for the wireless charger. Primary converters and transmitting coils are installed at charging stations, and receiving coils are installed underneath the electric vehicles.

We will study by analysis, circuit simulation, and hardware validation various converter topologies that are suitable for integration with different traction drive system architectures and that can achieve substantial reductions in the number of semiconductor devices and passive components. In the topology study, we will pursue maximization of the benefits that result from the higher operating frequencies and lower losses of WBG devices, especially GaN switches, and advanced soft magnetic materials such as nanocomposites. Resonant circuits for wireless chargers tend to produce currents and voltages several times higher than those needed for power transmission; thus they require costly components that can withstand high current and voltage stresses. Developing control strategies and optimal circuit designs that can minimize the resonant current and voltage is therefore another important aspect of this research.

FY 2015 work focuses on (1) developing converter topologies suitable for integration with various traction drive systems by reviewing and comparing published electric drive systems and simulating new topologies, and (2) improving wireless charging efficiency via design optimization for converter and resonant circuits with optimal control strategies to reduce resonant current.

This work builds upon ORNL's previous work on traction drives, onboard chargers (OBCs), and add-on wireless chargers. ORNL has demonstrated a $6.8 \mathrm{~kW}$ bidirectional SiC-based isolation converter that has a built-in $2 \mathrm{~kW} 14 \mathrm{~V}$ buck converter with a peak efficiency of $99 \%$, and a bidirectional $6.8 \mathrm{~kW} \mathrm{SiC-based} \mathrm{OBC}$ and dc-dc converter that enables a $47 \%$ reduction in component number for the power circuit components alone. The latter also enables savings in the gate driver and control logic circuits and provides a peak charging efficiency of $96.5 \%$ at $240 \mathrm{~V}$ input (Figure 3). 

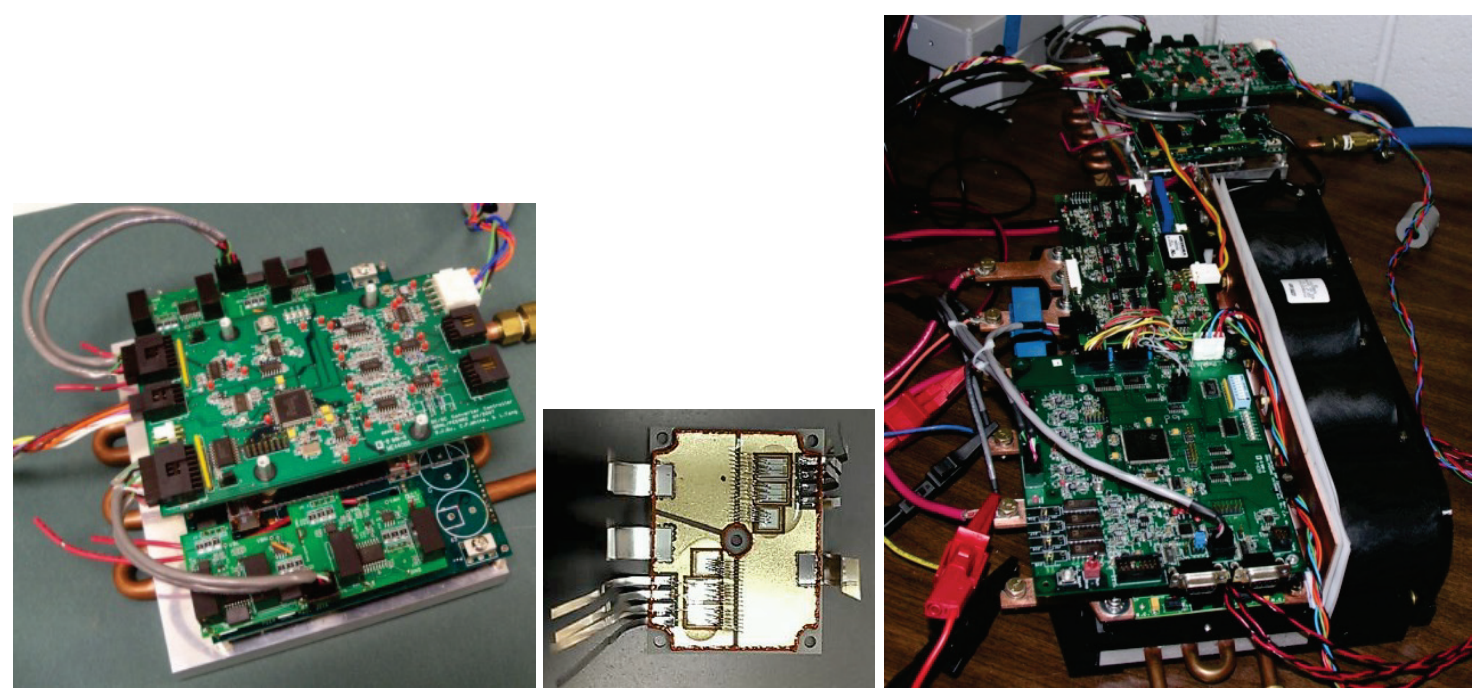

Figure 3: ORNL's previous work on traction drive, onboard charger: $6.8 \mathrm{~kW} \mathrm{SiC}$ isolation converter (left), ORNL SiC module $1200 \mathrm{~V} / 100 \mathrm{~A}$ (middle), SiC traction drive inverter with an integrated $6.8 \mathrm{~kW}$ OBC (right).

ORNL has demonstrated several add-on wireless chargers for both stationary and in-motion applications (Figures 4 and 5). The ORNL approach employs a series resonant circuit that operates at $22-26 \mathrm{kHz}$ and has high voltage gain. A high-frequency transformer is inserted in the primary side to adjust the gain for matching the output voltage to the vehicle battery. The ORNL work has been focused on antenna design and power transfer capability against misalignment of the antennas. It has demonstrated power transfer up to $10 \mathrm{~kW}$ continuous in full-scale laboratory test setups, with a peak efficiency of around $90 \%$.

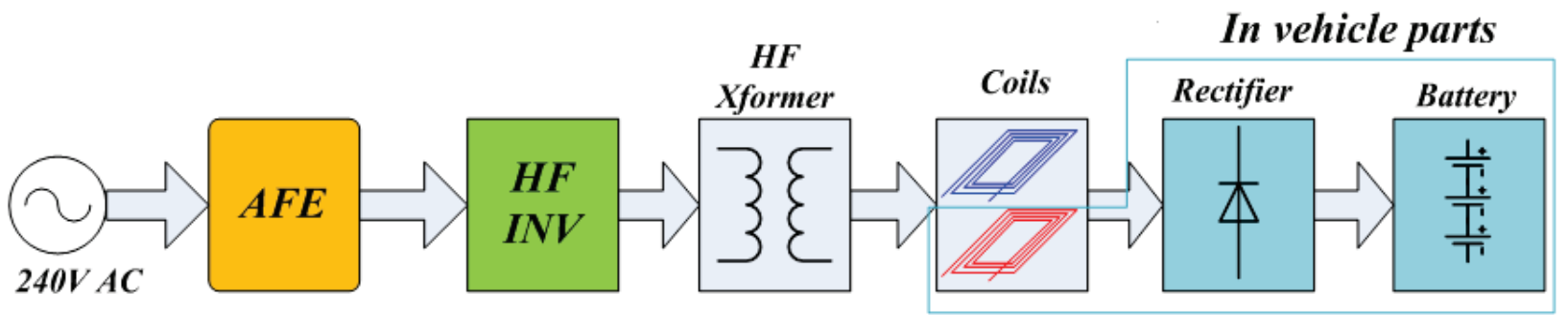

Figure 4: ORNL's add-on wireless chargers operating at $22-26 \mathrm{kHz}$.
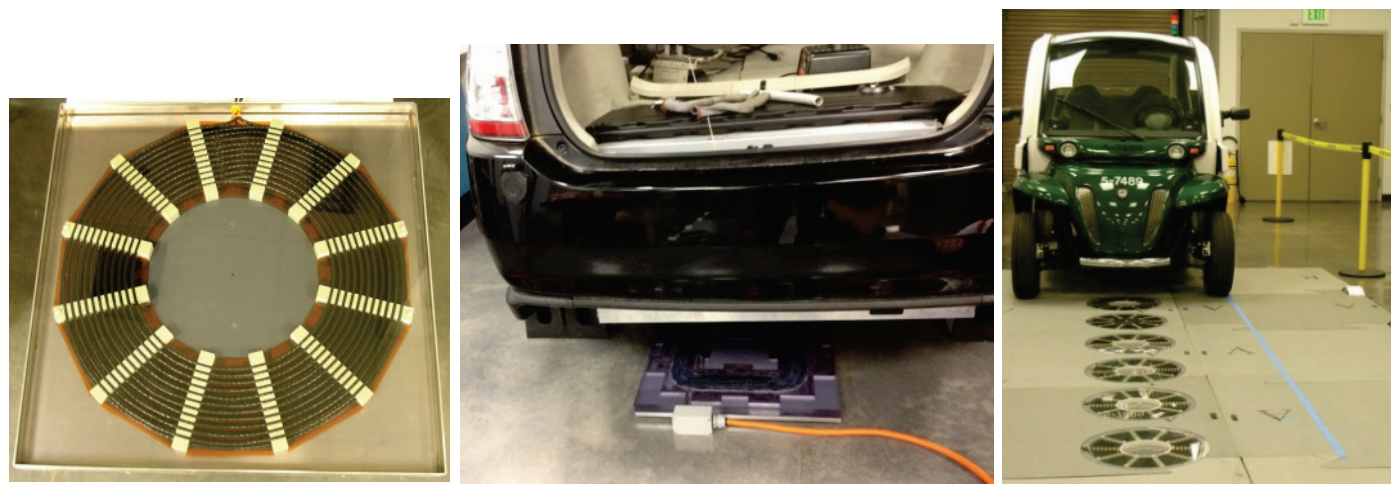

Figure 5: ORNL's previous work on add-on wireless chargers: coils (left), stationary wireless charging of a Prius PHEV (middle), in-motion wireless charging of a GEM EV (right).

Unique aspects of the approach are (1) leveraging ORNL's extensive past and current work on wired and wireless chargers and traction drives to redesign the electric drive system with wireless charging functionality; and (2) using ORNL's expertise and facility in converter design, packaging, and testing to fully take advantage of WBG devices in PEV applications. 
The impacts of the ORNL work are (1) successful demonstration of an electric drive system that incorporates wireless charging and WBG devices, providing a technological path to commercialization; and (2) a low-cost, high-efficiency electric drive system with wireless charging.

\section{Results and Discussion}

Inductor (L) and capacitor (C) resonant circuits are employed in wireless power transfer systems to increase the power transfer capability and efficiency between primary and secondary coils and minimize the supply voltage and current ratings by achieving soft-switching operation in the converters. There are four basic combinations of resonant topologies between the primary and secondary windings for wireless power transfer systems: series-series, series-parallel, parallel-series, parallel-parallel. In general, series resonance on the secondary side leads to a constant voltage source, while parallel resonance on the secondary side produces a constant current source.

Various LC resonant circuit designs that use modified circuits based on the four basic combinations were studied by simulation using the simplified de charger converter in Figure 6, and one design was selected that can minimize the $\mathrm{H}$-bridge inverter reactive power requirement by increasing load power factor and reducing circulating current.

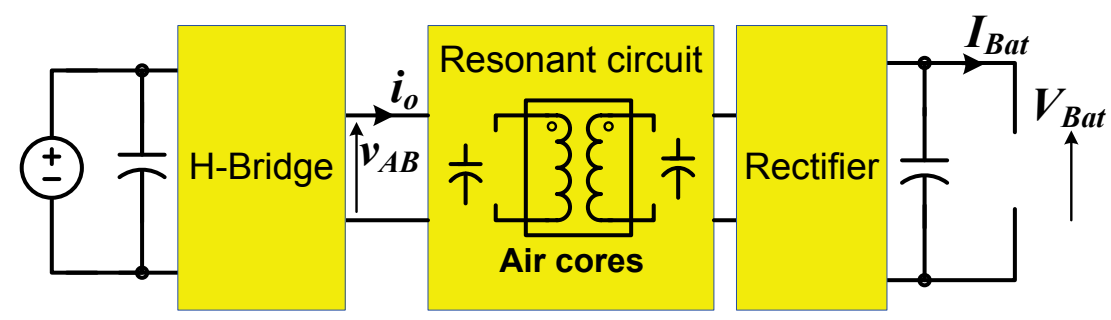

Figure 6: Simplified converter block diagram for studying resonant circuits.

Figure 7 shows a comparison of operating waveforms between a traditional resonant circuit with low load power factor (left) and the optimized resonant circuit with high load power factor (right). The traditional resonant circuit has a high primary current of io $=64.3 \mathrm{Vrms}$, compared with 19.3 Arms for the optimized resonant circuit.

The lower primary current levels and high power factors with the optimized resonant circuit lead to lower losses in the dc charger. For a comparison of losses and primary currents between the two resonant circuits, Figure 8 plots the ratios of the losses and primary current of the optimized resonant circuit against those of the traditional low-power-factor resonant circuit at various charging rates. The chart shows a range of $61-75 \%$ reduction in current and $10-44 \%$ reduction in losses.

Three electric drive topology candidates that have integrated wireless charging functionality and employ the optimized LC resonant circuit were studied using a detailed circuit simulation software package, which proved these concepts:

- Topology 1: Tapping into the $14 \mathrm{~V}$ accessory power supply converter to eliminate the secondary ac-dc converter for the wireless charger

- Topology 2: Using the traction motor and inverter to eliminate the secondary ac-dc converter for the wireless charger

- Topology 3: Using a multiport dc-dc converter that combines a boost converter of reduced power for stepping up the dc bus voltage of the traction drive inverter, a $14 \mathrm{~V}$ buck converter for powering the $14 \mathrm{~V}$ vehicle accessory loads, and a wireless battery charging converter 

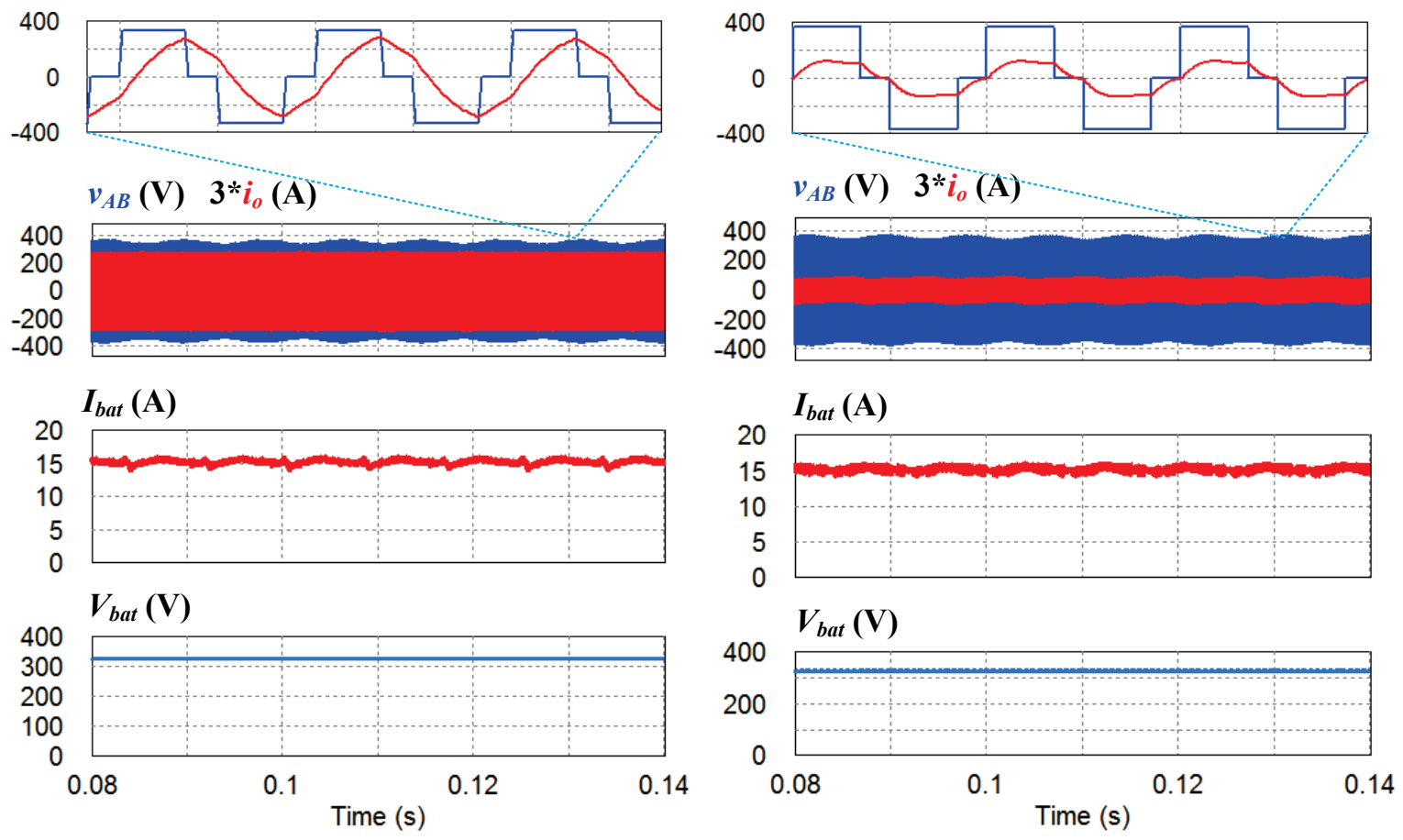

Figure 7: Comparison of operating waveforms between a traditional resonant circuit with low load power factor (left) and the optimized resonant circuit with high load power factor (right).

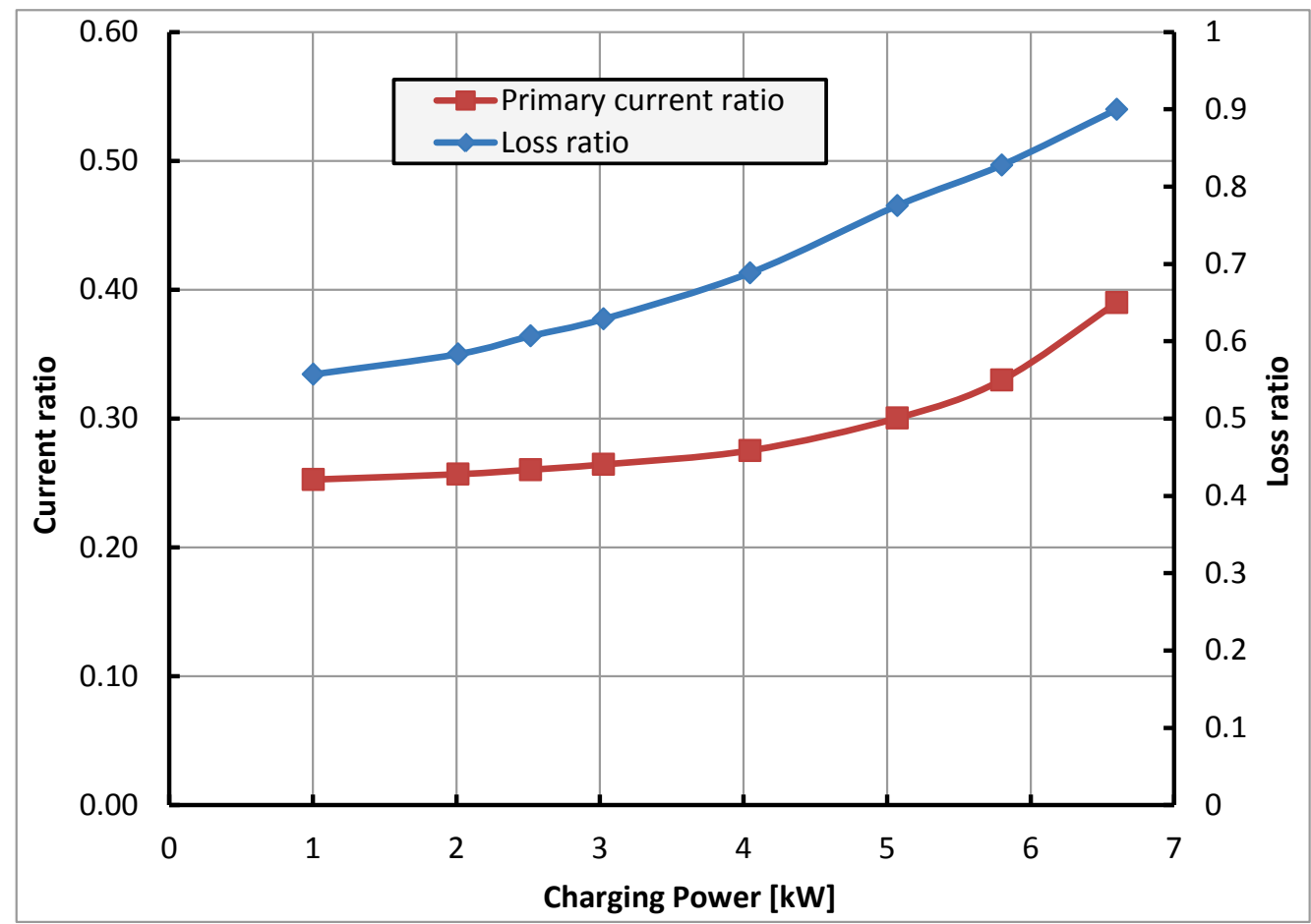

Figure 8: Comparison of losses and primary currents between the optimized resonant circuit with high power factor and the traditional circuit with low power factor at various charging rates.

Figure 9 shows a high-level block diagram of the first topology that taps into the $14 \mathrm{~V}$ accessory power supply converter to eliminate the need for a secondary ac-dc converter for the wireless charger. Figure 10 shows simulation results for charging at $1.5 \mathrm{~kW}$ from a $120 \mathrm{~V}$ ac source. It gives a high input power factor of $99.6 \%$ and a low THD of $1.1 \%$ in the ac source current. 


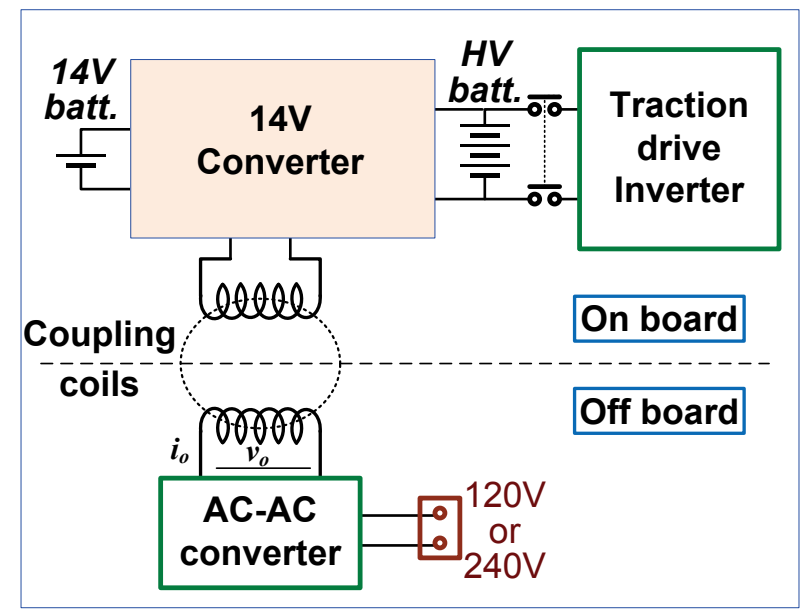

Figure 9: Simulated topology 1: tapping into the accessory power supply $14 \mathrm{~V}$ converter.

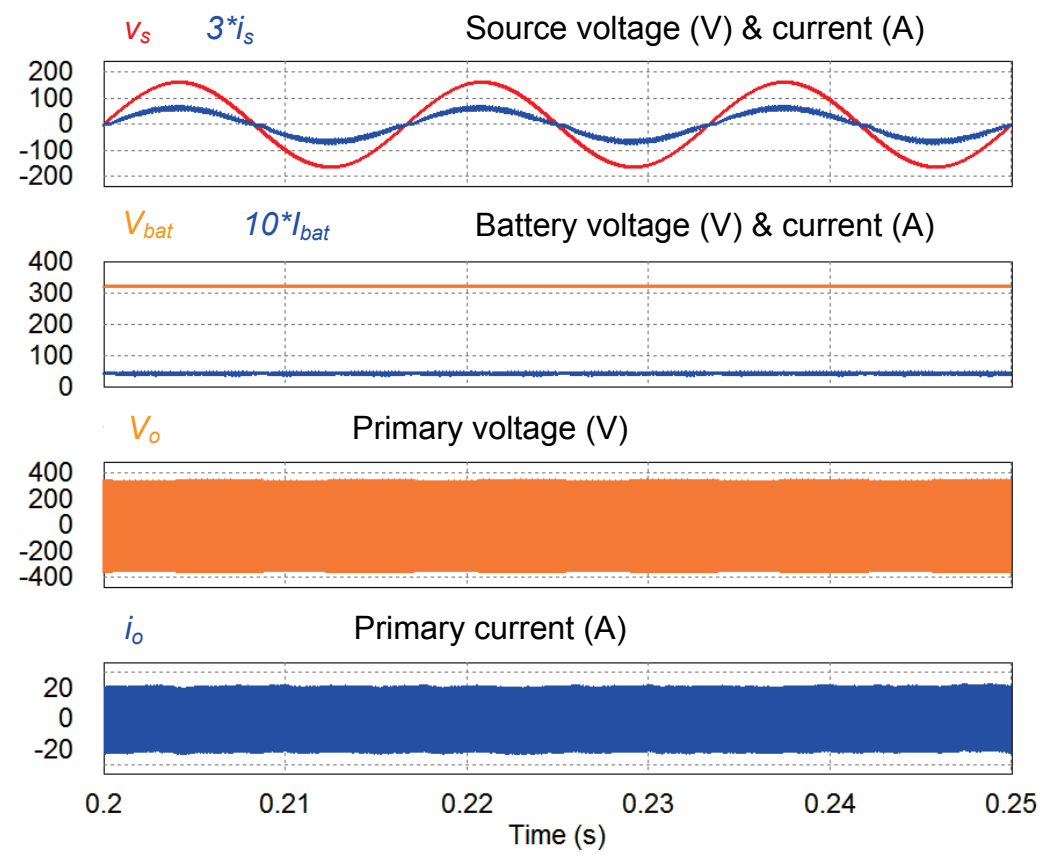

Figure 10: Simulation results for topology 1 for charging at $1.5 \mathrm{~kW}$ from a $120 \mathrm{~V}$ ac source, showing a high input power factor of $99.7 \%$ and a low THD of $1.2 \%$.

Figure 11 shows a simplified block diagram of the second topology that uses the traction motor and inverter to eliminate the need for a secondary ac-dc converter for the wireless charger. Figure 12 shows simulation results for charging at $5 \mathrm{~kW}$ from a $240 \mathrm{~V}$ ac source. It gives a high input power factor of $99.7 \%$ and a low THD of $1.2 \%$ in the ac source current. 


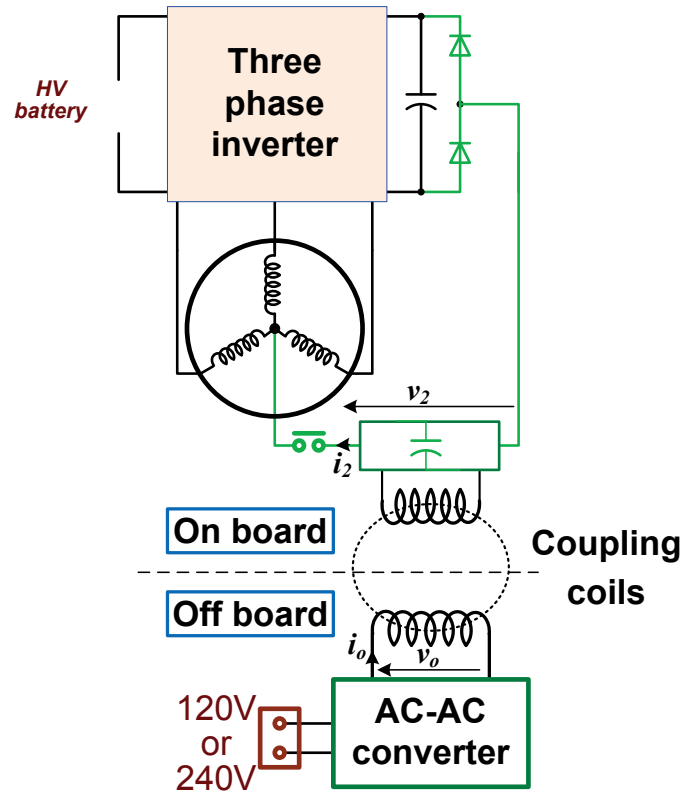

Figure 11: Simulated topology 2: using the traction motor and inverter.
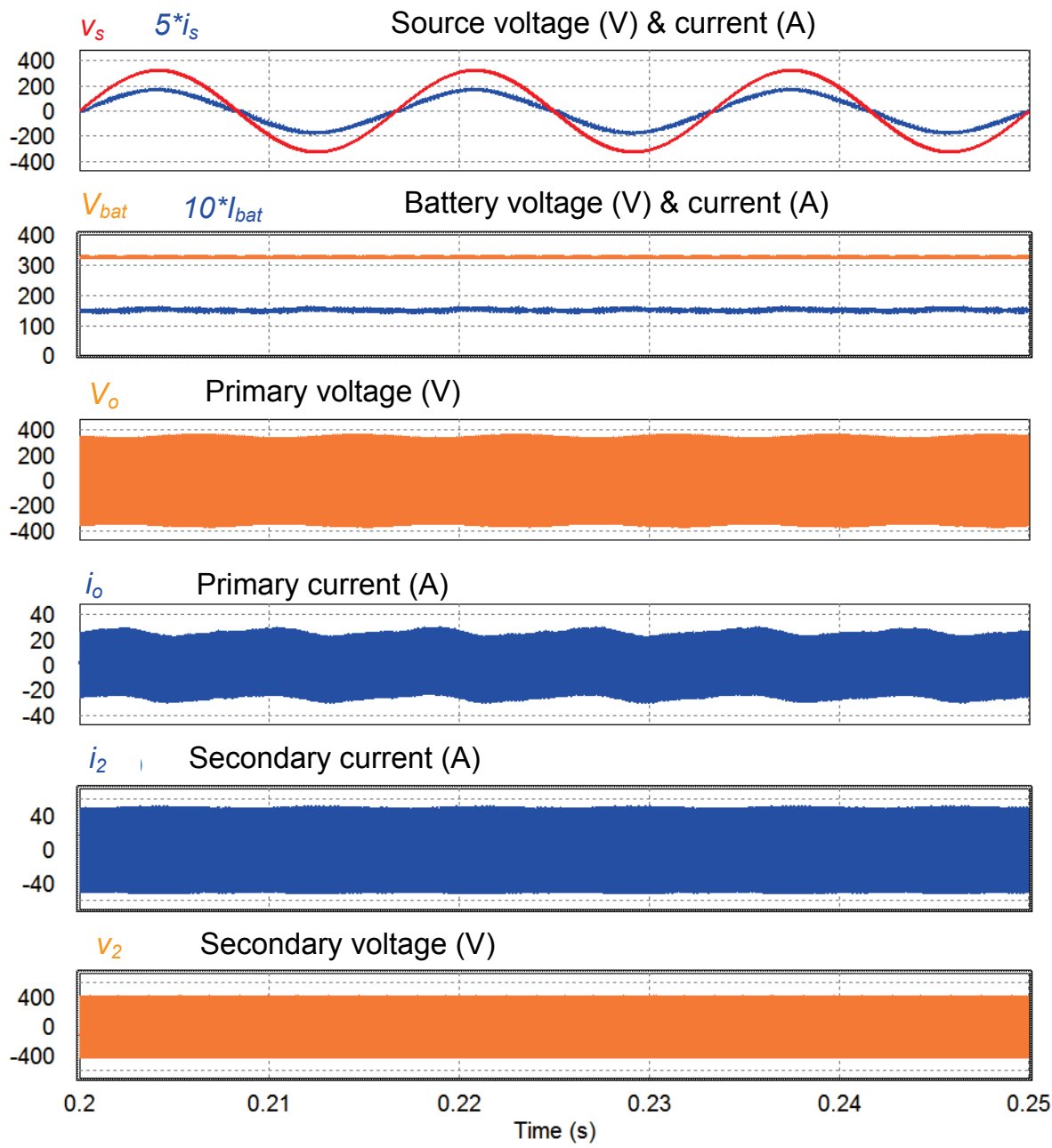

Figure 12: Simulation results for topology 2 for charging at $5 \mathrm{~kW}$ from a $240 \mathrm{~V}$ source, showing a high input power factor of 99.5\% and a low current THD of $0.8 \%$. 
Figure 13 shows a high-level block diagram of the third topology that employs a multiport dc-dc converter combining a reduced-power boost converter for stepping up the dc bus voltage of the traction drive inverter, a $14 \mathrm{~V}$ buck converter for powering the $14 \mathrm{~V}$ vehicle accessory loads, and a wireless battery charging converter.

Figure 14 gives simulation results for topology 3 charging the battery at the rated power of $6.6 \mathrm{~kW}$ from a $240 \mathrm{~V}$ ac source. It gives a high input power factor of $99.73 \%$ and a low THD of $1.1 \%$ in the ac source current. Figure 15 gives simulated operating waveforms for battery voltage, inverter dc link voltage (boosted from the battery at 250 to $650 \mathrm{~V}$ ), and inverter 3-phase output currents during operation in propulsion mode at an inverter output power level of $50 \mathrm{kVA}$.

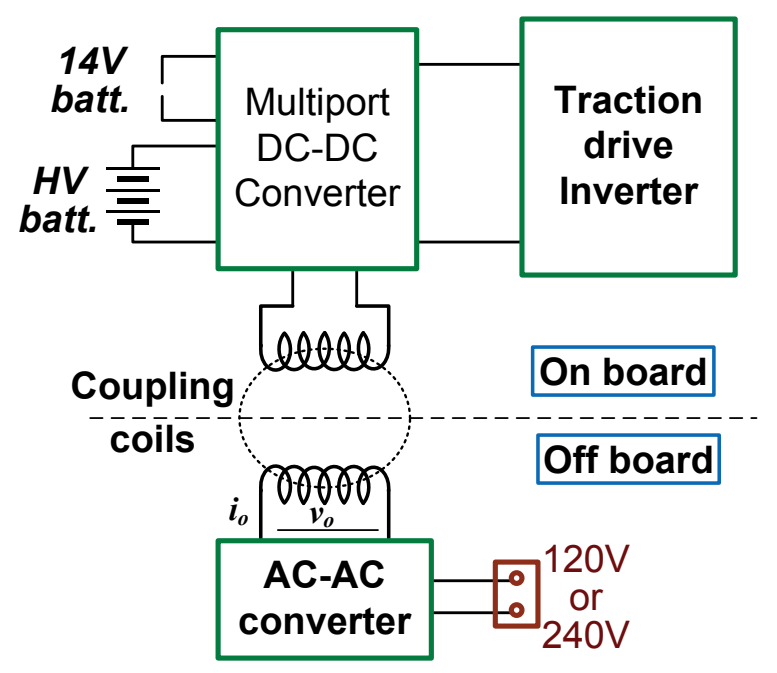

Figure 13: Simulated topology 3: using a multiport dc-dc converter that combines a boost converter of reduced power for stepping up the dc bus voltage of the traction drive inverter, a $14 \mathrm{~V}$ buck converter for powering the $14 \mathrm{~V}$ vehicle accessory loads, and a wireless battery charging converter.
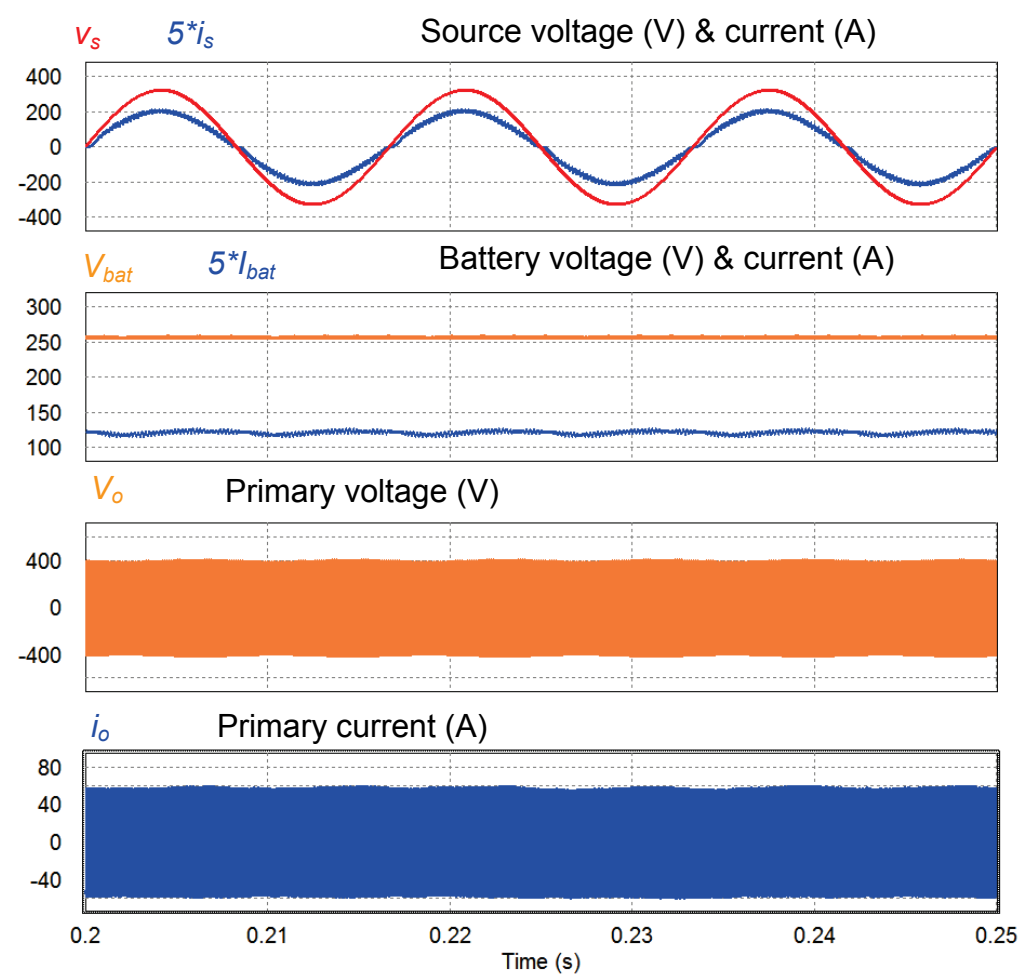

Figure 14: Simulated operating waveforms for topology 3 for charging at $6.6 \mathrm{~kW}$ from a $240 \mathrm{~V}$ source, showing high input power factor and low current distortion. 


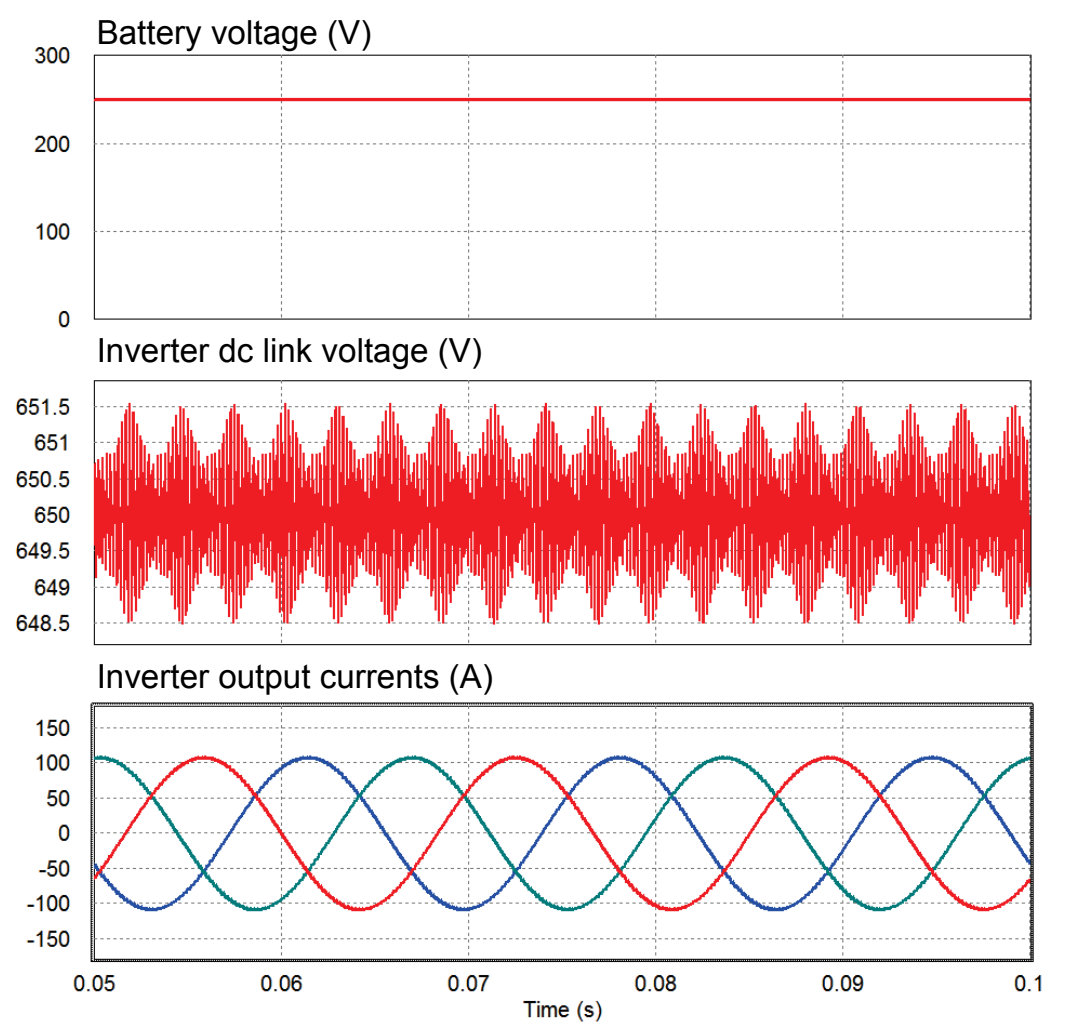

Figure 15: Simulated operating waveforms for topology 3 operating in propulsion mode.

Figure 16 plots simulated power factor and THD against charging power for topology 3 . It shows high input power factors of greater than $99 \%$ and low THD factors of less than $3.5 \%$ in the ac source current.

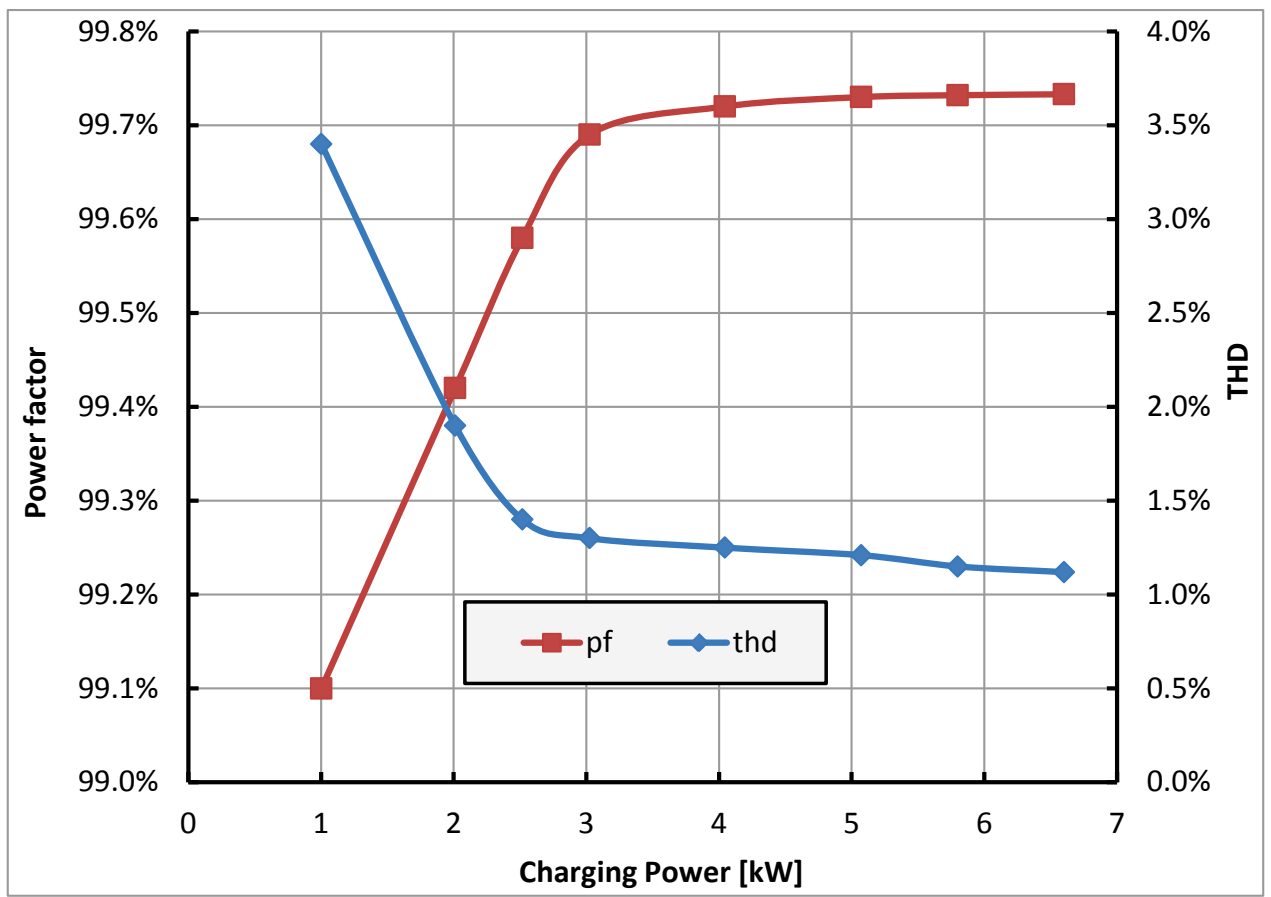

Figure 16: Simulated power factor and THD against charging power for topology 3. 


\section{Conclusions and Future Direction}

This project aims at redesigning the electric drive system for PEVs to provide wireless charging as an integral function at low cost and high efficiency. It does so by minimizing the PEV system component numbers and cost through functional integration of onboard power electronics for vehicle propulsion and wireless battery charging. In addition, increased efficiency and further reduced weight and volume are achieved by optimizing the converter topology and coil design and using WBG devices.

FY 2015 work generated a high-power-factor resonant circuit that was found effective in reducing the circulating current and the associated losses. Simulation results showed reductions in the range of $61-75 \%$ for the current and $10-44 \%$ for the losses.

FY 2015 work also produced and proved concepts for three electric drive topologies with integrated wireless charging functionality. Simulation results showed all the wireless chargers have high input power factors of greater than $99 \%$ and low THD factors of less than $3.5 \%$ in the ac source current.

Future work includes (1) design, build, and testing of WGB-based prototypes and (2) once proof-of-concept hardware development is complete, collaboration with and/or technology transfer to industry.

\section{FY 2015 Presentations/Publications/Patents}

1. G. J. Su, "Traction drive systems with integrated wireless charging," presented at the DOE Vehicle Technologies Office Electric Drive Technologies Advanced Power Electronics and Electric Motors R\&D FY 2015 Kickoff Meeting, Oak Ridge, Tennessee, November 18-20, 2014.

2. G. J. Su, "Traction drive systems with integrated wireless charging," presented at the 2015 DOE Hydrogen and Fuel Cells Program and Vehicle Technologies Office Annual Merit Review and Peer Evaluation Meeting, June 8-12, 2015. 


\title{
3.4 Gate Driver Optimization for WBG Applications
}

\author{
M. Nance Ericson, Principal Investigator \\ Oak Ridge National Laboratory (ORNL) \\ National Transportation Research Center \\ 2360 Cherahala Boulevard \\ Knoxville, TN 37932 \\ Phone: (865) 574-5637 \\ E-mail: ericsonmn@ornl.gov
}

Susan A. Rogers, DOE EDT Program Manager

Phone: (202) 586-8997

E-mail: Susan.Rogers@ee.doe.gov

Burak Ozpineci, ORNL EDT Program Manager

Phone: (865) 946-1329

E-mail: burak@ornl.gov

Contractor: UT-Battelle, LLC, managing and operating contractor for the Oak Ridge National Laboratory Contract No.: DE-AC05-00OR22725

\section{Abstract/Executive Summary}

This project addresses a technology gap resulting from the consequences of repeated fast current and voltage transitions in wide bandgap (WBG) -based drive systems and the deleterious effect they can have on system and motor reliability over time. By providing enhanced control and optimization of the gate drive, through dynamic closed-loop slew rate limiting of di/dt (instantaneous current change rate) and $\mathrm{dv} / \mathrm{dt}$ (instantaneous rate of voltage change over time), fast-switching WBG-based drive systems can achieve higher system reliability, power density, and efficiency, leading to increased market acceptance at a lower system cost point.

This project will further the minimization and integration of the electronics required for traction motor drive systems. An optimized gate drive for $\mathrm{SiC}$ metal-oxide-semiconductor field-effect transistors (MOSFETs) will be designed, fabricated, and tested in two phases. The first prototype will be built in a benchtop circuit using off-the-shelf components and its operation will be evaluated. This will be followed by the second phase of the project, focusing on the development of the gate drive in a monolithic integrated circuit, advancing highfrequency WBG design-based size reduction benefits.

\section{Accomplishments}

All tasks were completed during FY 2015 within budget. All reporting and presentation requirements were met.

- Research of Existing Methods

- A review of the scientific literature dealing with state-of-the-art methods of performing $\mathrm{di} / \mathrm{dt}$ and $\mathrm{dv} / \mathrm{dt}$ control was performed. Three primary protection methods were identified: solid state circuit breaker, fault current evaluation, and desaturation detection. Of these methods, desaturation detection was chosen to be the most appropriate, particularly for approaches with a goal of maximized monolithic integration.

- Of the active gate drive methods identified, source inductance-based current measurement was the common method of choice. All methods addressed silicon devices (insulated gate bipolar transistors [IGBTs] and MOSFETs), with none addressing the specific needs of driving SiC devices. Furthermore, all methods appeared monolithically compatible, but none was found that had been implemented as an integrated circuit or integrated circuit chipset. 
- di/dt was established as the most appropriate measured parameter from this literature search, and a review of conventional means for current measurement was performed, specifically for measuring the drain current of a power MOSFET (see Table 1).

- Current measurement using a sense inductor was determined to be the overall best choice for motor drive applications.

- Results of the reviewed methods and architectures for active gate drives are summarized in Table 2, with an emphasis on the topology and measured parameter (di/dt, dv/dt).

- Gate Driver Design and Architecture Selection

- Multiple gate drive waveforms were investigated and simulations performed using SPICE to predict efficacy in controlling the $\mathrm{di} / \mathrm{dt}$ and $\mathrm{dv} / \mathrm{dt}$ of a MOSFET switching a motor leg.

- A double pulse test setup was constructed and used to evaluate the transient switching characteristics of commercially available SiC MOSFET devices from Cree. Using data from these tests, some SPICE model adjustments were made and simulations re-run.

- It was determined that improved SPICE models were needed for more accurate dynamic simulation of the WBG devices.

- To address the SiC MOSFET modeling need, a private company was identified that has expertise in $\mathrm{SiC}$ circuit modeling and simulation. A commercial simulation tool (CoolSPICE) was acquired, along with a few $\mathrm{SiC}$ powerMOS device models for evaluation.

- Gate Driver Design Simulations

- A new gate drive waveform was developed (variable threshold) and was shown via simulation to provide the desired control of the transient switching characteristics. This method also automatically compensates for variation in the powerMOS threshold voltage from device to device and over temperature.

- Simulations of the closed-loop gate driver were performed in PSPICE using vendor-supplied SiC powerMOS device models (C2M0080120D, Cree Inc.). The loop control set point was stepped to demonstrate the range of operation. Some simplifications were used to improve the simulation convergence, including the use of a single-slope gate drive waveform. Results showed the loop operated in a stable fashion.

- Enhanced modeling activities with CoolSPICE software were begun and will be carried forward in year 2 of the project.

The results demonstrated the efficacy of the ORNL-developed sensing and control approach and will serve as the beginning point for the physical prototyping of an advanced gate drive circuit in year 2 of the project.

\section{Introduction}

WBG devices, primarily $\mathrm{SiC}$ and $\mathrm{GaN}$, have characteristics that can lead to dramatic benefits in a variety of power electronics applications. These include higher-temperature and higher-frequency operation, smaller device and overall system sizing, and greater operational efficiency. Their ability to switch at higher frequencies than their silicon counterparts can also lead to significant volume reduction in associated passive components, yielding second-order benefits in reduced material costs and weights. For every $10 \%$ reduction in the weight of a vehicle, a 6-7\% increase in fuel economy can be realized. For this reason, significant attention has been given to the miniaturization and lightweighting of all components and systems in a vehicle by automotive original equipment manufacturers. The rapidly escalating move from the use of power silicon to WBG devices to capture some of these advantages entails revisiting and optimizing their interplay with ancillary circuits and systems to truly exploit their unique characteristics.

Available commercial gate drives are not optimized for use with WBG devices. They have historically been developed for silicon devices and cannot exploit the distinctions that WBG semiconductors can offer traction drive systems. The faster di/dt and dv/dt switching capabilities of WBG devices can impose implications that need to be addressed at a system level. Fast switching can reduce the reliability of motor insulation by creating pinholes in the insulation that eventually can cause arcing and failure of the motor. To combat this possibility, 
additional costs must be incurred for higher-rated winding insulation, imposing increased motor and cabling costs and resulting in larger and heavier motors. Additionally, fast switching transients can cause pitted bearings, resulting in unwanted circulating currents. Fast voltage and current transitions can also necessitate added electromagnetic interference (EMI) filtering requirements to protect sensitive electronic systems from electronic interference, adding further costs and increased system volume.

This project addresses the cost and volume barriers to achieving the DOE traction system goals of $\$ 8 / \mathrm{kW}$ and $>4 \mathrm{~kW} / \mathrm{L}$ power density. To achieve these goals, a cost-effective, efficient topology for monitoring and controlling the dynamic slew rate of a WBG gate drive will be developed to drive a SiC MOSFET motor leg. The design will be implemented in an integrated circuit to minimize the volume and reduce packaging issues.

This work can serve as an enabling technology, which, if implemented in a high-temperature process, can be utilized for future integrated motor/inverter systems.

The design will result in

- Higher inverter reliability and efficiency by monitoring and utilizing dynamic slew control

- $50 \%$ reduction in gate drive electronics volume by drive integration

- Higher temperature capability $\left(>200^{\circ} \mathrm{C}\right)$, by use of silicon-on-insulator (SOI) technology

- Higher motor reliability

- Reduced insulation breakdown

- Reduced bearing currents

- Lower-cost gate drive systems through parts reduction

- Gate driver integration

- Decreased EMI filtering

This project will advance the technology of gate drives and hasten the deployment of WBG devices in vehicular traction drives by significantly increasing power density and lowering cost, circuit volume, and weight while increasing reliability and allowing the opportunity to move toward an integrated, hightemperature-capable traction system.

\section{Approach}

An active gate driver topology was developed to address the technology gaps associated with WBG-specific gate drives. This architecture was simulated using a custom-designed circuit topology with a vendor-supplied WBG device model in PSPICE. The three tasks identified for carrying out the project goals are the following:

- $\quad$ Task 1. Research Existing Methods

- Evaluate existing methods for slew rate limiting/control for feasibility and for implementation in an integrated circuit.

- Investigate both commercially available methods and those in the open literature.

- Task 2. Gate Driver Design and Architecture Selection

- Perform an iterative gate drive architecture design considering potential cost and system-level performance implications.

- Task 3. Gate Driver Design Simulations

- Optimize and finalize the gate driver architecture via iterative circuit simulation.

Previous work had been performed on a VTO-funded project with the University of Tennessee on an SOI integrated gate driver design. However, it did not incorporate active switching. Circuits constructed and tested under that project included the following: 
- Input level translator

- Shoot-through protection

- Protection circuits

- Thermal shutdown

- Under-voltage lockout

- Short circuit detection

- Desaturation

- Gate current

- Integrated voltage regulator

The project strategy was to incorporate lessons learned from that project, as well as synergies with other previous projects focused on $\mathrm{SiC}$ gate drivers; incorporate slew rate control; and ultimately develop a hightemperature-capable gate driver integrated circuit suitable for integration with a traction motor to realize size, volume, and DOE cost targets.

The implementation of an active gate drive was divided into two broad categories, and the pros and cons of each topology were examined.

1. Gate voltage control—gate voltage is fed through a resistor from an amplifier or buffer

- Implementation advantages
- Simple
- Voltage amplifier can be used
- Can be used to control both di and dv loops

- Implementation disadvantages

- Amplifier/resistor combination needs to be able to handle both on and off switching, which likely requires different set points

- Gate voltage is dropped across a resistor

2. Gate current control—current through a resistor is monitored

- Implementation advantages

- Actual gate voltage is sensed

- More opportunity for control with greater complexity

- Can be used to control both di and dv loops

- Implementation disadvantages

- More complex

- Potentially harder to compensate

Following a careful analysis of options suitable for incorporation into an integrated circuit gate driver design, tasks were combined with modeling and simulation activities. These activities resulted in the selection of a closed-loop circuit architecture. This topology was designed with several requirements in mind: ability to handle the high bandwidth of SiC MOSFET switches, practical and effective sensing of di/dt and dv/dt, potential to use only a single feedback parameter, and suitability for monolithic integration.

\section{Results and Discussion}

A literature review was performed to investigate common current measurement methods. Each of the methods was evaluated with an emphasis on parameters important to incorporation of the technique into a gate drive feedback system. These parameters included insertion loss, the need for external power, degree of electrical isolation, signal bandwidth, physical size, measurement accuracy, and relative cost compared with other conventional current measurement techniques (see Table 1). Of the methods investigated, the use of a sense inductor collectively met all of the requirements for the intended application, except accuracy. The accuracy limitation is primarily due to the relatively low inductance value generally used $(5-10 \mathrm{nH})$. However, the method is receiving significant industry attention, and a number of $\mathrm{SiC}$ powerMOS devices are now packaged to provide separate current sense and source power pins (a Kelvin connection). Furthermore, this method is the 
most commonly reported for current measurement in active gate drive circuits (see Table 2), was very costeffective, and was selected for use in this program.

Table 1: Summary of conventional current measurement methods (sources: Allegro STP98-1-AN, Rev.2, Pearson 2878 data sheet)

\begin{tabular}{|l|l|l|l|l|l|l|l|}
\hline \multicolumn{1}{|c|}{ Sensor type } & $\begin{array}{c}\text { Insertion } \\
\text { loss }\end{array}$ & $\begin{array}{l}\text { External } \\
\text { power }\end{array}$ & $\begin{array}{c}\text { Circuit } \\
\text { isolation }\end{array}$ & \multicolumn{1}{|c|}{ Bandwidth } & Size & Accuracy & $\begin{array}{c}\text { Relative } \\
\text { cost }\end{array}$ \\
\hline Sense resistor & High & Low & Low & DC to $50 \mathrm{MHz}$ & Medium & Medium & Low \\
\hline Sense inductor & Low & Low & Low & DC to $50 \mathrm{MHz}$ & Small & Low & Low \\
\hline $\begin{array}{l}\text { Open-loop Hall } \\
\text { effect }\end{array}$ & Low & Low & High & DC to $>100 \mathrm{kHz}$ & Small & Medium & Medium \\
\hline $\begin{array}{l}\text { Closed-loop } \\
\text { Hall effect }\end{array}$ & Low & Medium & High & DC to $1 \mathrm{MHz}$ & $\begin{array}{l}\text { Medium/ } \\
\text { large }\end{array}$ & Medium & Medium \\
\hline $\begin{array}{l}\text { Current } \\
\text { transformers }\end{array}$ & Medium & None & High & $30 \mathrm{~Hz}$ to $70 \mathrm{MHz}$ & $\begin{array}{l}\text { Medium/ } \\
\text { large }\end{array}$ & $\begin{array}{l}\text { Low- } \\
\text { High }\end{array}$ & High \\
\hline
\end{tabular}

Table 2: Summary of published active gate drive methods

\begin{tabular}{|l|l|l|l|l|l|l|}
\hline \multicolumn{1}{|c|}{ Reference } & di/dt & dv/dt & Complexity & $\begin{array}{l}\text { i measurement } \\
\text { method }\end{array}$ & $\begin{array}{c}\text { Chip } \\
\text { compatible }\end{array}$ & \multicolumn{1}{|c|}{ Notes } \\
\hline Lobsinger $2012^{1}$ & $\mathrm{Y}$ & $\mathrm{Y}$ & Moderate & Le & $\mathrm{Y}$ & IGBT \\
\hline Lobsinger $2015^{2}$ & $\mathrm{Y}$ & $\mathrm{Y}$ & Moderate & Le & $\mathrm{Y}$ & IGBT \\
\hline Wang $2013^{3}$ & $\mathrm{Y}$ & $\mathrm{N}$ & Low & Le & $\mathrm{Y}$ & IGBT \\
\hline Park $2003^{4}$ & $\mathrm{Y}$ & $\mathrm{Y}$ & Low & Le & $\mathrm{Y}$ & IGBT/MOSFET \\
\hline Gerster $1996^{5}$ & $\mathrm{Y}$ & $\mathrm{Y}$ & Low & Le & $\mathrm{Y}$ & IGBT \\
\hline $\begin{array}{l}\text { Riazmontazer } \\
2015^{6}\end{array}$ & $\mathrm{Y}$ & $\mathrm{Y}$ & Moderate & - & $\mathrm{Y}$ & IGBT/MOSFET, \\
\hline Chen $2009^{7}$ & $\mathrm{Y}$ & $\mathrm{Y}$ & Low & Le & $\mathrm{Y}$ & IGBT \\
\hline
\end{tabular}

Previous VTO-funded collaborative research between ORNL and the University of Tennessee was reviewed to identify opportunities to leverage it. This previous research included chip-level design of an input level translator, shoot-through protection, various protection circuits, and an integrated voltage regulator. The primary candidate protection methods are compared in Table 3. A desaturation detection method was chosen for future implementation based on its functionality and suitability for monolithic integration. These methods will be further reviewed and finalized during the chip integration activities of year 3 . 
Table 3: Comparison of three candidate protection methods

\begin{tabular}{|l|l|l|l|}
\hline \multicolumn{1}{|c|}{$\begin{array}{c}\text { Protection } \\
\text { method } \\
\text { features }\end{array}$} & SSCB & \multicolumn{1}{|c|}{ Desaturation } & $\begin{array}{l}\text { Fault current } \\
\text { evaluation }\end{array}$ \\
\hline Fault response & Fast & Fast & Fast \\
\hline Power dissipation & Hery Good & Good & Very low \\
\hline Reliability & Medium & High & Hood \\
\hline Implementation complexity & High & Low & Medium \\
\hline Integration complexity & High & Low & Low \\
\hline Cost & & & \\
\hline
\end{tabular}

A literature review was performed to investigate closed-loop active gate drive (AGD) architectures, with an emphasis on the type of feedback ( $\mathrm{di} / \mathrm{dt}$ and/or $\mathrm{dv} / \mathrm{dt}$ ), implementation complexity, current measurement method, chip compatibility, and application space (e.g., Si IGBT, Si MOSFET, WBG MOSFET). These methods were entirely directed toward silicon IGBT and MOSFET switching devices, except in Riazmontazer et al. (2015), who used a predictive, somewhat complex method solely based on drain-to-source $\mathrm{dv} / \mathrm{dt}$ for driving SiC MOSFETs.

A clamped inductor test system was constructed to facilitate double pulse testing of commercial $\mathrm{SiC}$ power MOSFETs. Second- and third-generation devices from CREE (C2M0080120 and C3M0065090, respectively) were tested using this setup, and the measurement results were compared with PSPICE simulations using vendor-supplied device simulation models.

The initial proposed AGD topology for this work is based on di/dt feedback using a sensing inductor, as shown in Figure 1. Since the bandwidth requirements for pulse-by-pulse gate control are impractically high, a topology was chosen that averages a number of cycles to produce the needed gate drive for $\mathrm{di} / \mathrm{dt}$ control. Means of sampling the peak di/dt on both the rising and falling transitions are shown, and each is low-pass filtered and fed to the slew generator block for adjusting the subsequent gate drive slew characteristics. Sampling and control are synchronous operations that are controlled by the timing generator block. The output gate buffer provides large source and sink currents required for the gate drive, and protection circuits (undervoltage-lockout and desaturation) are included for monitoring and drive disabling.

Multiple gate drive waveforms were investigated via PSPICE simulation and the results were compared for the purpose of maximizing di/dt and dv/dt control, including single-slope (variable slew), RC (variable RC time constant), dual slope (fixed threshold, variable slew to opposite rail), and threshold control (fast transition to a plateau voltage - hold for a period of time, then transition rapidly to the opposite rail). For the latter, note that on the turn-on transition, the plateau voltage is above the device threshold voltage; and for the turn-off transition, the plateau voltage is below the device threshold voltage. Of these waveforms (shown in Figure 2), threshold control was found to provide the optimum drive, in terms of maximized $\mathrm{di} / \mathrm{dt}$ and $\mathrm{dv} / \mathrm{dt}$ dynamic range and minimized delay variation. One added advantage of this technique is that it compensates for variations in the switching device threshold voltage and associated temperature dependence. Hybrids of these control methods are possible - including varying the hold time, varying the slew rate during the hold period, and control of the fast control edge slew rates - which may be investigated, time permitting. A summary of the PSPICE simulation results for the threshold gate drive waveform method is shown in Figure 3. The simulated $\mathrm{di} / \mathrm{dt}$ and $\mathrm{dv} / \mathrm{dt}$, shown as a function of the voltage level (plateau voltage) for both turn-on and turn-off transitions, predict reasonable control of these important transient parameters using this waveform method. 


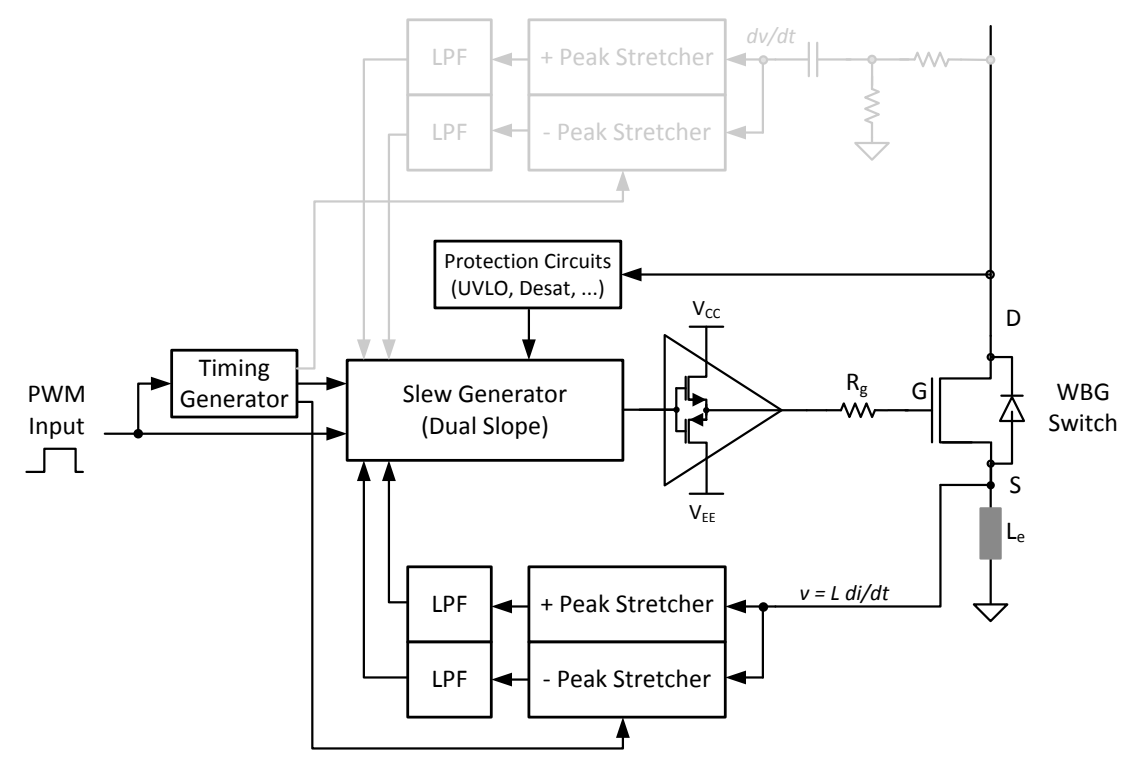

Figure 1: General active gate drive topology selected incorporating WBG source inductive current sensing for di/dt feedback control. Optional dv/dt sensing method is also shown for completeness.

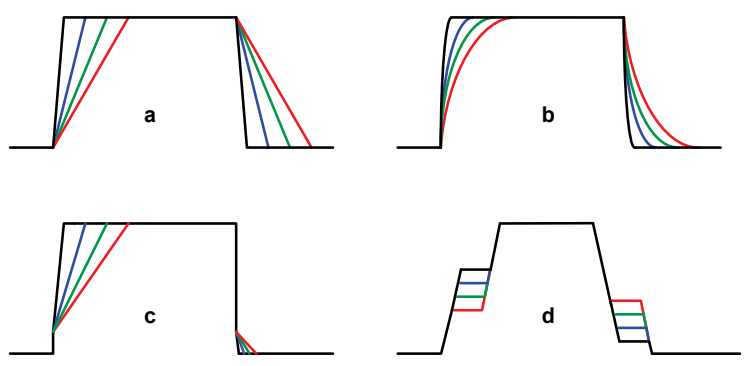

Figure 2: Candidate gate drive waveforms for di/dt and dv/dt control: (a) single slope, (b) RC-based, (c) dual slope, and (d) threshold method.
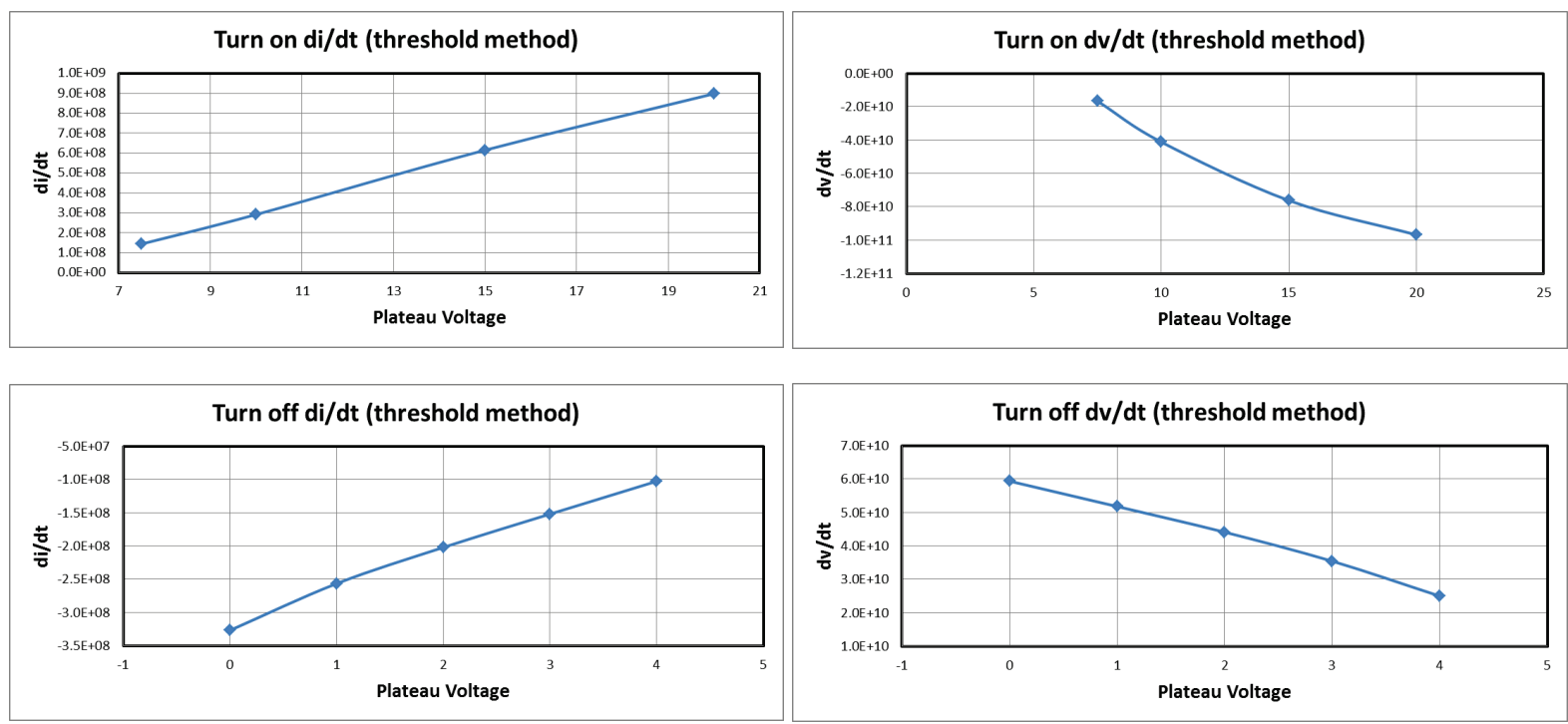

Figure 3: Simulated di/dt (left column) and dv/dt (right column) produced using the threshold gate drive waveform during SiC powerMOS device turn-on (top row) and turn-off (bottom row).

After further simulation, the AGD topology was refined for closed-loop simulation using PSPICE (see Figure 4). For simplicity, the single slope drive method (Figure 2a) was used, as it provided a straightforward 
slew generator implementation with good convergence characteristics. The positive peak of the source sensing inductor di/dt was sampled, low-pass filtered, and compared with a reference set point, and the error signal was used to modify subsequent gate drive cycles. For these simulations, an $8 \mathrm{kHz}$ input signal was used, as is common for a pulse-width-modulated (PWM) drive signal in traction drive inverters. In this sampled system, the di/dt generated error signal changes only every $125 \mu$ s (or every PWM period). Careful attention was given to this loop to produce stable operation. Figure 5 shows a closed-loop simulation while the $\mathrm{di} / \mathrm{dt}$ set point is changed at four different values. These closed-loop simulations indicate the ability to control the di/dt and $\mathrm{dv} / \mathrm{dt}$ using di/dt-based feedback from a source inductance. Further refinement of the topology is ongoing, including implementation of the threshold gate drive circuit and further optimization of the feedback loop to improve stability and response time. These results will serve as a good starting point for a bench prototype gate driver to be constructed using commercial off-the-shelf components, tested, and optimized in year 2 of the program.

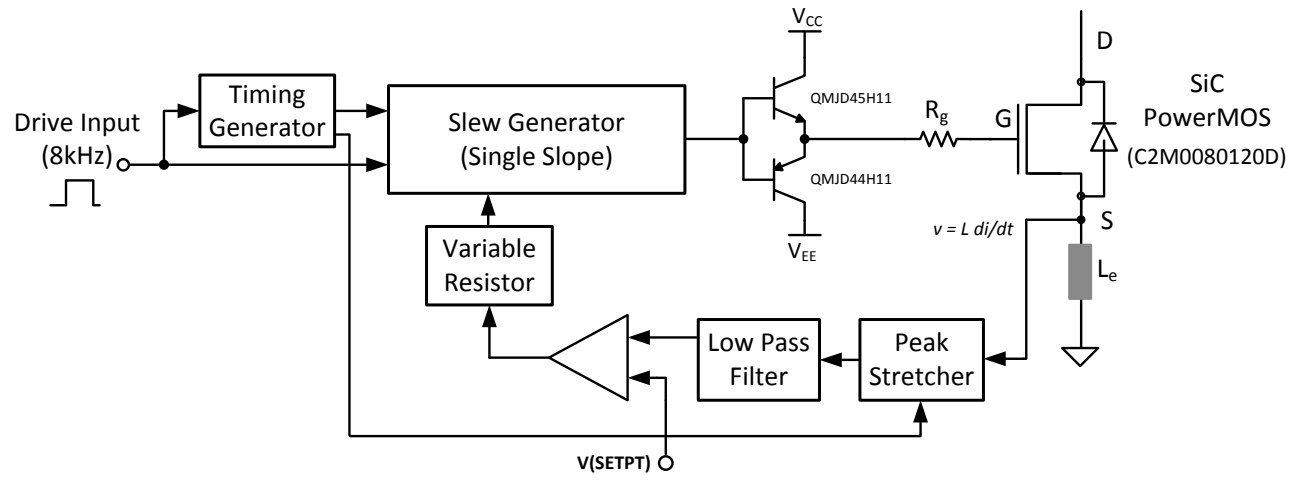

Figure 4: Closed-loop PSPICE simulation showing dynamic changing of the di/dt loop control set point and subsequent loop settling. The switch frequency chosen is $8 \mathrm{kHz}$.

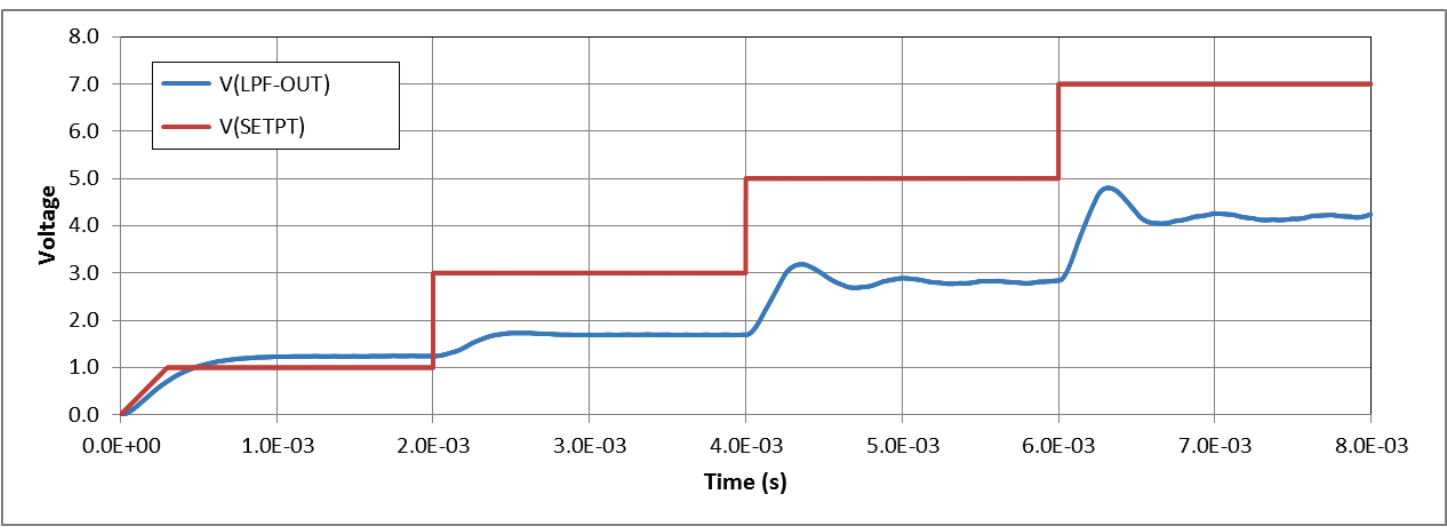

Figure 5: Closed-loop PSPICE simulation showing dynamic changing of the di/dt loop control set point and subsequent loop settling. The switch frequency chosen is $8 \mathrm{kHz}$ and the dynamic range of the di/dt for this simulation is $\sim 3 \times$.

\section{Conclusions and Future Direction}

This project is focused on achieving the DOE volume, weight, and cost targets for the traction drive inverter while enabling a high-temperature solution for an integrated motor/inverter system. It proposes to develop a circuit to actively monitor and optimally respond to the fast switching characteristics of SiC switches to ensure system reliability, enabling safe use of SiC MOSFETs in drive inverter legs to increase inverter efficiency. In year 2, work will begin on incorporating the circuit into an integrated circuit.

Tests of WBG devices were performed to construct/optimize models for use in simulation activities during the year. Extensive simulations were carried out during FY 2015, leading to iterative model modifications and 
simulations. This effort emphasized the importance of having precise WBG device models, and an improved simulation tool and associated SiC power MOSFETs models are being acquired to assist with the research.

The past year's work has culminated in the development of an improved architecture for actively controlling the gate drive of an inverter $\mathrm{SiC}$ power switch. Multiple waveform types were evaluated by simulation, and an improved waveform was developed that is actively modulated using a di/dt sensing inductor. To handle the extremely fast switching behavior of WBG devices, a slower control loop is used, rather than high bandwidth. Single pulse modulation is commonly used in silicon-based gate drive applications. This topology and associated implementation were developed with the intention of incorporating it into an integrated circuit for minimizing size, weight, cost and volume.

Further simulation activities will be performed during FY 2016 to more accurately optimize the gate drive architecture, including the drive waveform specifics and compensation of the control loop. Simulation efforts will transition to the newly acquired CoolSPICE for closed-loop, system-level simulations with optimized SiC powerMOS device models (provided by CoolCAD Inc.). A board-level prototype of the active gate driver will be constructed and bench tested with an inductive load. This prototype will enable further testing and optimization of the circuit, including the drive waveform, feedback parameters, and associated sensing, before the device is transitioned to an integrated circuit.

With the maturation of WBG devices and their penetration into the automotive sector, it is expected that a more system-level approach to their implementation will be needed in the years to come. Optimization of the gate drive and its packaging will be paramount in these efforts. This research will position the ORNL team to initiate SOI integrated circuit implementation of the AGD an enabling technology for realizing reliable and highly compact WBG-based traction drive systems.

\section{FY 2015 Presentations/Publications/Patents}

1. N. Ericson, C. Britton, L. Marlino, S. Frank, D. Ezell, L. Tolbert, and J. Wang, "Gate Driver Optimization for WBG Applications," DOE VTO Electric Drive Technologies FY 2015 Kickoff Meeting, Knoxville, Tennessee, November 18, 2014.

2. N. Ericson, C. Britton, L. Marlino, S. Frank, D. Ezell, L. Tolbert, J. Wang, and B. Blalock, "Gate Driver Optimization for WBG Applications," USCAR EE Tech Team Project Update Meeting, Southfield, Michigan, March 26, 2015.

3. N. Ericson, C. Britton, L. Marlino, S. Frank, D. Ezell, L. Tolbert, and J. Wang, "Gate Driver Optimization for WBG Applications," DOE Annual Merit Review, Washington, DC, June 10, 2015.

\section{References}

1. Y. Lobsiger and J. W. Kolar, "Closed-loop IGBT gate drive featuring highly dynamic di/dt and dv/dt control," in IEEE Energy Conversion Congress and Exposition, 4754-4761, September 2012.

2. Y. Lobsiger and J. W. Kolar, "Closed-loop di/dt and dv/dt IGBT gate driver," IEEE Transactions on Power Electronics 30(6), 3402-3417, June 2015.

3. Z. Wang, X. Shi, L. M. Tolbert, B. J. Blalock, "Switching performance improvement of IGBT modules using an active gate driver," in IEEE Applied Power Electronics Convergence, 266-1273, March 2013.

4. S. Park and T. M. Jahns, "Flexible dv/dt and di/dt control method for insulated gate power switches," IEEE Transactions on Industry Applications 39(3), 657-664, May/June 2003.

5. C. Gerster and P. Hofer, "Gate-controlled dv/dt and di/dt limitation in high power IGBT converters," EPE Journal 5(3/4), 11-16, January 1996.

6. H. Riazmontazer, A. Rahnamaee, A. Mojab, S. Mehrnami, S. K. Mazumber, M. Zefran, "Closed-loop control of switching transition of SiC MOSFETs," in IEEE Applied Power Electronics Conference, 782-788, March 2015.

7. L. Chen, F. Z. Peng, "Closed-loop gate drive for high power IGBTs," in IEEE Applied Power Electronics Conference, 1331-1337, February 2009. 


\subsection{Benchmarking EVs and HEVs}

\section{Tim Burress, Principal Investigator}

Oak Ridge National Laboratory (ORNL)

National Transportation Research Center

2360 Cherahala Boulevard

Knoxville, TN 37932

Phone: (865) 946-1216

E-mail: burressta@ornl.gov

\section{Susan A. Rogers, DOE EDT Program Manager}

Phone: (202) 586-8997

E-mail: Susan.Rogers@ee.doe.gov

\section{Burak Ozpineci, ORNL EDT Program Manager}

Phone: (865) 946-1329

E-mail: burak@ornl.gov

Contractor: UT-Battelle, LLC, managing and operating contractor for the Oak Ridge National Laboratory Contract No.: DE-AC05-00OR22725

\section{Abstract/Executive Summary}

Information from the Benchmarking project provides crucial information about the status of and trends in electric vehicle/hybrid electric vehicle (EV/HEV) technologies to DOE VTO for strategic planning with respect to state-of-the-art progressions. ORNL's detailed benchmarking reports have received overwhelmingly positive feedback from researchers in academia and industry, as well as other individuals with an interest in $\mathrm{EV} / \mathrm{HEV}$ technologies. This information serves as a valuable educational resource for EV/HEV architectures and yields a track of lessons learned, prevents reinventing/duplication of advancements, and promotes "leapfrog"/competitive development.

\section{Accomplishments}

- Designed, fabricated, and assembled the hardware necessary to adapt the 2014 Honda Accord HEV transmission to the dynamometer test cell.

- Successfully implemented the integration of ORNL controls with the 2014 Accord power converter unit (PCU).

- Conducted comprehensive dynamometer testing of a 2014 Accord inverter and motor at 300, 500, and $700 \mathrm{Vdc}$ to obtain an efficiency map and many other performance metrics.

- Confirmed published peak torque and power specifications for a 2014 Accord inverter and motor.

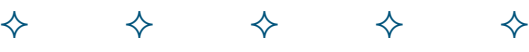

\section{Introduction}

The 2014 Honda Accord is a full hybrid vehicle that includes a motor with a published power rating of $124 \mathrm{~kW}$, which is much more powerful than previous-generation ratings of about 12 and $14 \mathrm{~kW}$. The hybrid system has many similarities to Toyota's Hybrid Synergy Drive, and the vehicle reportedly has the highest city fuel efficiency rating (49 MPG) of all hybrids in its class. This report reviews results from the initial disassembly of the transaxle, motor, and PCU and discusses detailed findings from teardown analyses. Comprehensive dynamometer testing was conducted and the performance, efficiency, and other operational results are summarized. 


\section{Approach}

Automotive manufacturers usually do not publish details about the design, functionality, and operation of $\mathrm{EV} / \mathrm{HEV}$ technologies, and even published details and specifications need to be verified and clarified. For example, single-value power ratings for motors and inverters are often published; but they do not include information about the power capability throughout the operation range (e.g., versus speed), the duration for which this power can be maintained, the efficiency throughout the operation region, and many other important characteristics. Therefore, ORNL performs teardown assessments to obtain comprehensive information on design, functionality, and sub-component characteristics. Furthermore, components are completely instrumented and tested in a dynamometer test cell to determine operational characteristics such as performance and efficiency. These activities provide the information needed for DOE to warrant a robust program and provide DOE partners and other researchers with valuable information on state-of-the-art EV/HEV technologies.

\section{Results and Discussion}

The new Accord hybrid system contains two 3-phase electric motor-generators. The power train can operate in full electric mode, series electric mode, and engine mode. In series electric mode, power from the engine is absorbed and supplied to the battery and drive motor. When cruising at highway speeds, the engine can be directly coupled through a fixed gear ratio to the drive wheels, facilitating higher-efficiency operation. A diagram of the new hybrid system is shown in Figure 1. Overall, the electrical system layout and configuration is nearly identical to that of the previous Toyota hybrid systems that have been benchmarked. The HEV and plug-in HEV models include batteries with nominal voltages of 259 and $320 \mathrm{~V}$, respectively. The HEV system includes a bidirectional boost (dc-dc) converter with a $411 \mu \mathrm{F}, 370 \mathrm{~V}$ capacitor on the input of the boost converter and a large $1,125 \mu \mathrm{F}, 700 \mathrm{Vdc}$ capacitor on the output of the boost converter. The battery voltage is boosted to a maximum level of $700 \mathrm{Vdc}$, and both motor and generator inverters are attached to the highvoltage bus.

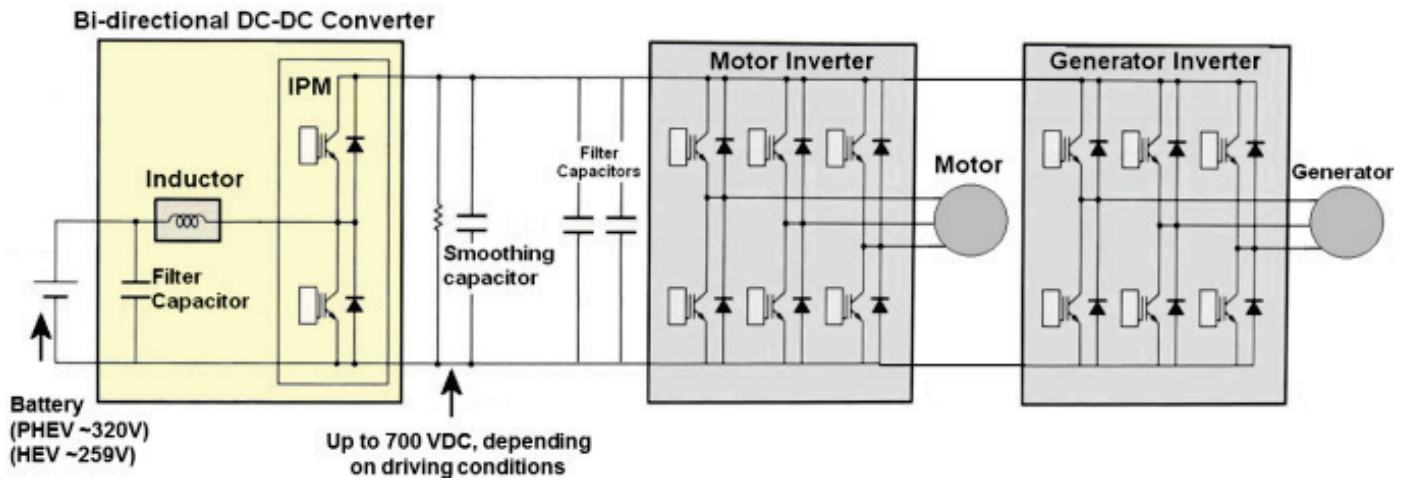

Figure 1: Diagram of the 2014 Honda Accord hybrid electrical system.

Exterior views of the 2014 Honda Accord HEV PCU are shown in Figure 2. It is manufactured by Fuji Electric, and the overall mass and volume are $17.5 \mathrm{~kg}$ and $12.4 \mathrm{~L}$, respectively. An oval connector on the PCU attaches to power cables from the battery, and two circular connectors attach to the 3-phase motor and generator. A 31-pin connector provides power to the PCU and, among many other functions, communication with other components on the vehicle. Cooling ports for the flow of standard ethylene glycol/water cooling are located at one end of the PCU, and another port is located on the opposite end to which the cooling system reservoir attaches.

The PCU has three main sections: the top compartment, bottom compartment, and heat sink located between them. As shown at right in Figure 3, the bottom compartment houses the capacitors, the inductor for the boost converter, a bleed resistor, and a current transducer for the boosted dc current. A white, zinc oxide-based thermal paste was observed on the bottom of the inductor, which is mounted to the bottom of the heat sink, but no other components in the bottom compartment are actively cooled. 

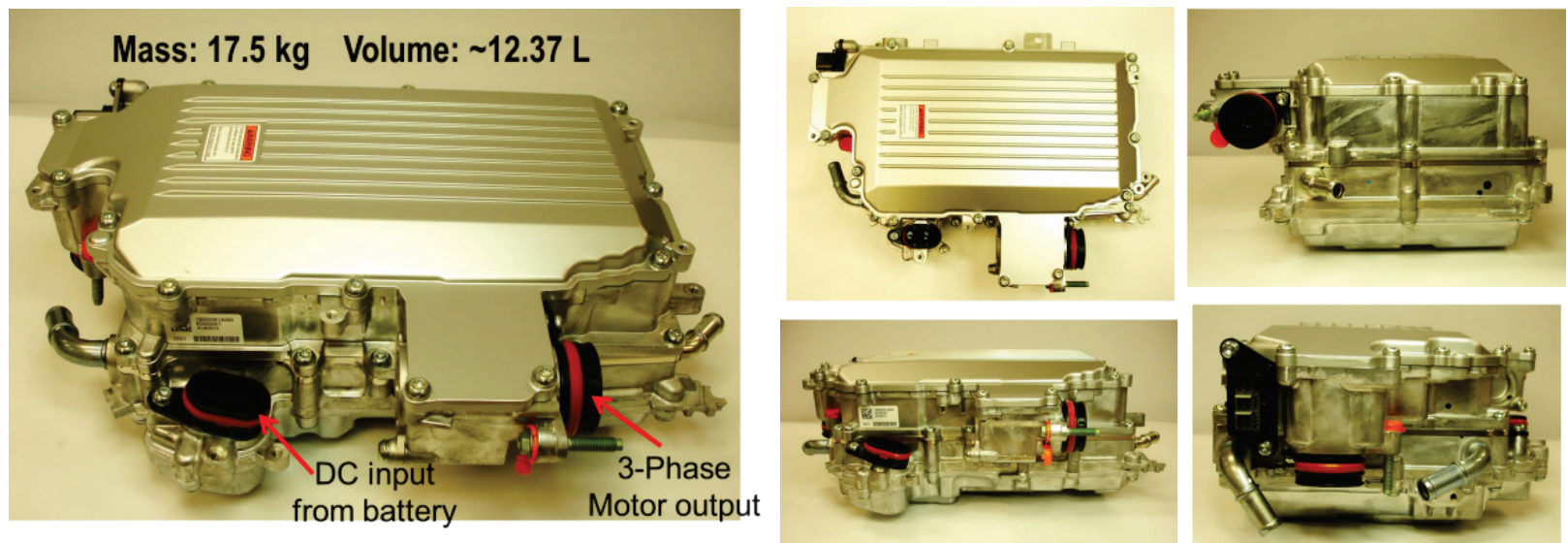

Figure 2: Various exterior views of the 2014 Honda Accord hybrid PCU.
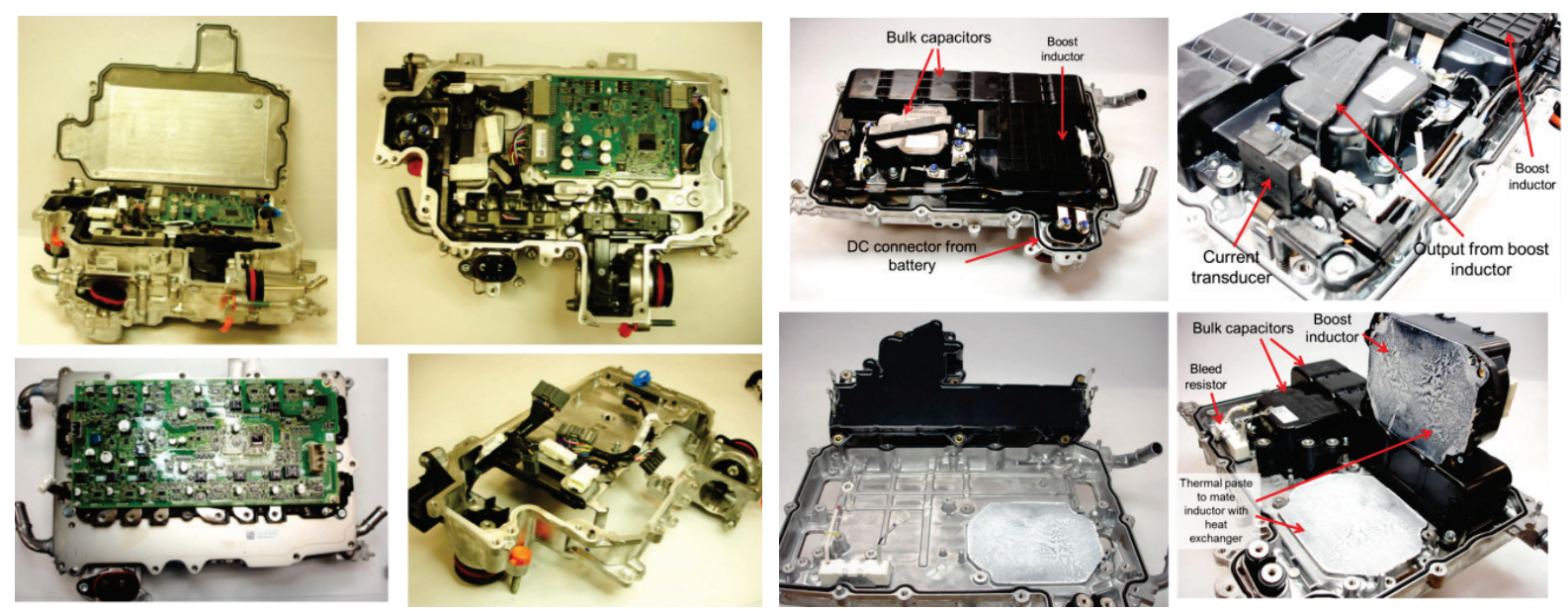

Figure 3: Upper compartment (left) and lower compartment (right) of 2014 Honda Accord PCU.

Shown at left in Figure 3 is the upper compartment, which includes the control board, power module, current transducers, and dc and ac bus bars from the power module to the exterior connectors. The control board is supported by a substantial plate that was cast as a part of the aluminum housing. After the driver board is removed from the power module, a planar electromagnetic interference (EMI) shield can be seen above the dc and ac bus bar infrastructure. In most Toyota power modules, the bus bars are more readily integrated within the module. The power module is integrated with the heat sink, which is shown at right in Figure 4. Upon removal of the driver board (shown at left in Figure 5), EMI shield, and bus bar structure, the power electronics devices are visible.
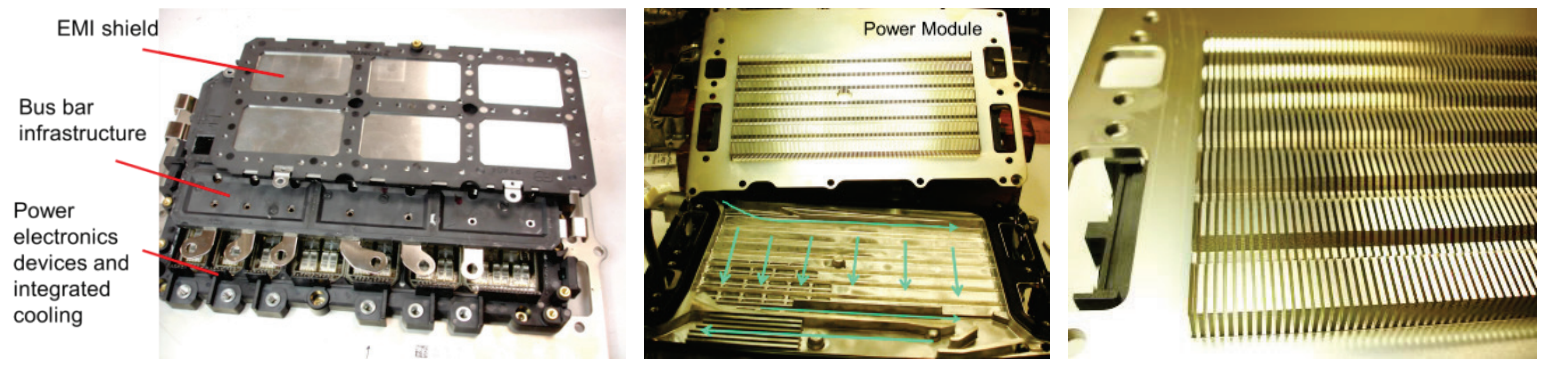

Figure 4: Accord power module periphery (left) and heat exchanger (right).

As shown at right in Figure 5, power devices for the generator inverter, motor inverter, and boost converter are located within the same module, a consolidation also observed in recent Toyota power modules. The generator inverter uses one insulated-gate bipolar transistor (IGBT) and one diode per switch, for a total of six IGBTs and six diodes. The motor inverter uses 2 IGBTs and 2 diodes per switch, giving a total of 12 IGBTs and 12 
diodes. The boost converter uses three IGBTs with two diodes for the lower switch, and two IGBTS and two diodes for the upper switch. All previously benchmarked boost converters contained the same number of IGBTs for upper and lower switches.
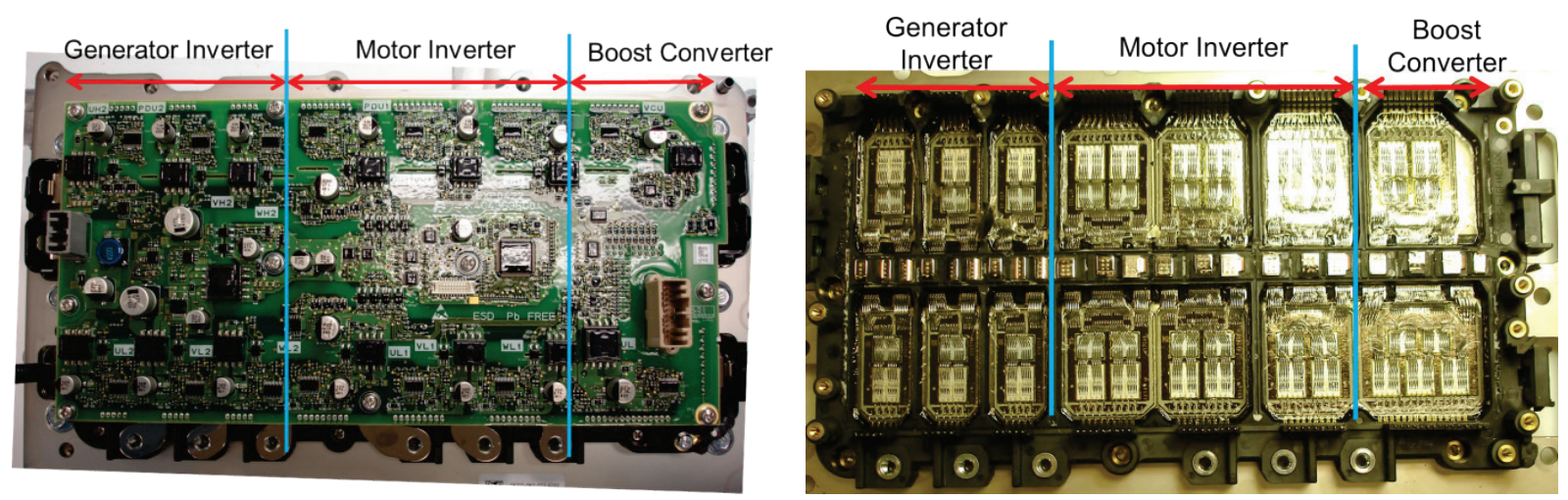

Figure 5: Accord power module with (left) and without (right) driver board.

Dimensions of the IGBTs and diodes are labeled at left in Figure 6. All IGBTs and diodes in the PCU have the same dimensions: approximately $12.18 \times 15.21 \mathrm{~mm}$ and $12.15 \times 11.06 \mathrm{~mm}$, respectively. Material compositional analysis was conducted to determine the materials used within the power module. The center image in Figure 6 indicates the overall stack-up of the power module; it shows that IGBT and diode power devices are soldered to a direct-bond copper (DBC) cladding about $1.12 \mathrm{~mm}$ thick. The solder layer thickness is $\sim 0.13-0.14 \mathrm{~mm}$. The upper copper cladding is attached to an $\sim 0.32 \mathrm{~mm}$ thick silicon nitride ceramic dielectric that serves as an electrical insulator for the IGBT and diode. A more commonly used lower-cost ceramic dielectric is aluminum nitride; but a few other hybrid systems, including the Lexus LS 600h, also have used silicon nitride. The ceramic dielectric is attached to another copper-clad layer that is about $0.40 \mathrm{~mm}$ thick. The lower copper-clad layer is soldered to the nickel-plated aluminum baseplate/heat sink. The nickel plating is about $10 \mathrm{um}$ thick and the lower solder layer is about $0.40 \mathrm{~mm}$ thick. Nickel plating appears to cover the entire baseplate/heat sink shown in Figure 4.
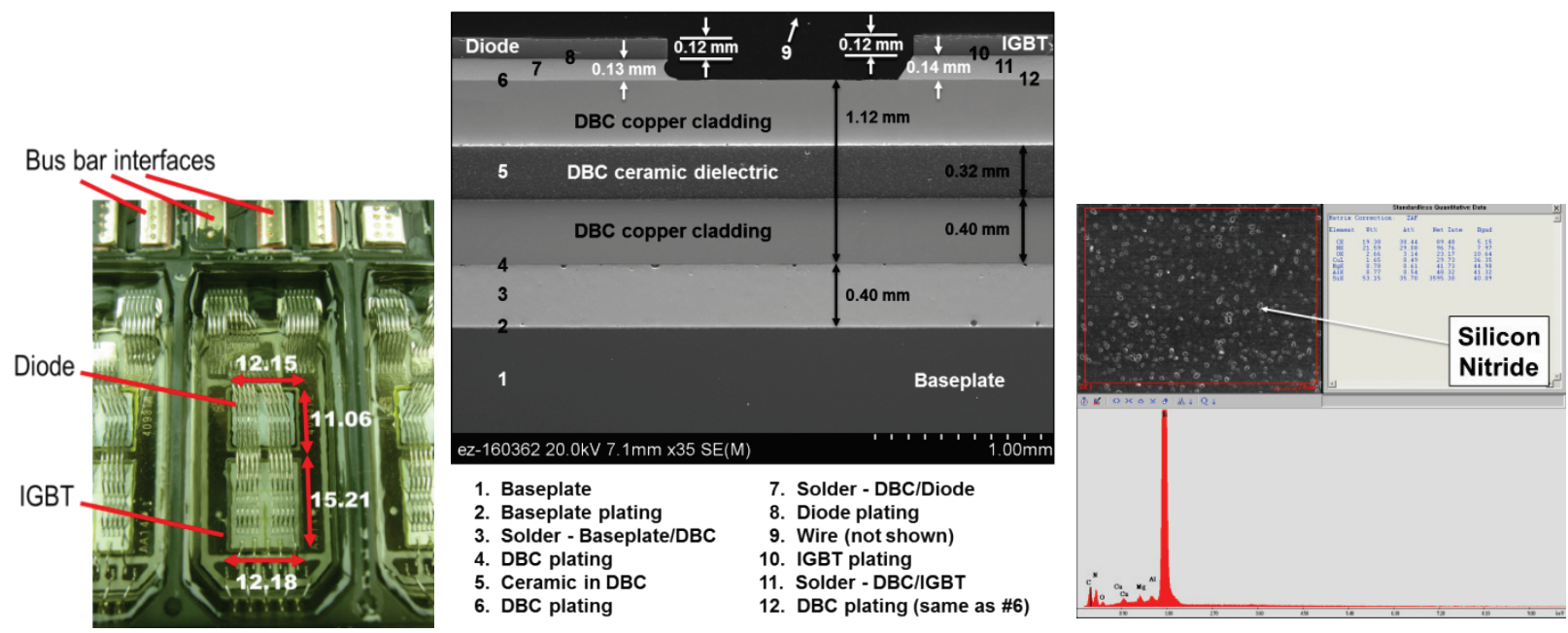

Figure 6: Accord IGBT and diode (left) and power module stack-up (right).

The 2014 Accord transmission, shown in Figure 7, has a total mass of about $113.5 \mathrm{~kg}(249.5 \mathrm{lb})$, which is quite close to the 2007 Camry hybrid transmission mass of $108 \mathrm{~kg}$. This is a good comparison because both passenger vehicles are sedans of similar size and power requirements. As is the case for many hybrid transmissions, there is no torque converter between the internal combustion engine and the transmission. Other than the absence of a torque converter, the transmission mounts to the engine in a conventional manner with a flywheel attached to the spline. Various sections of the transmission are indicated at right in Figure 7, where the engine input spline shafted is labeled. The gear section is adjacent to the engine mounting location and 
includes the differential gear that drives the left and right drive shafts and ultimately the front wheels. The section that houses the drive motor is located next to the gear section, and the generator section is located next to the motor section on the opposite end of the transmission.
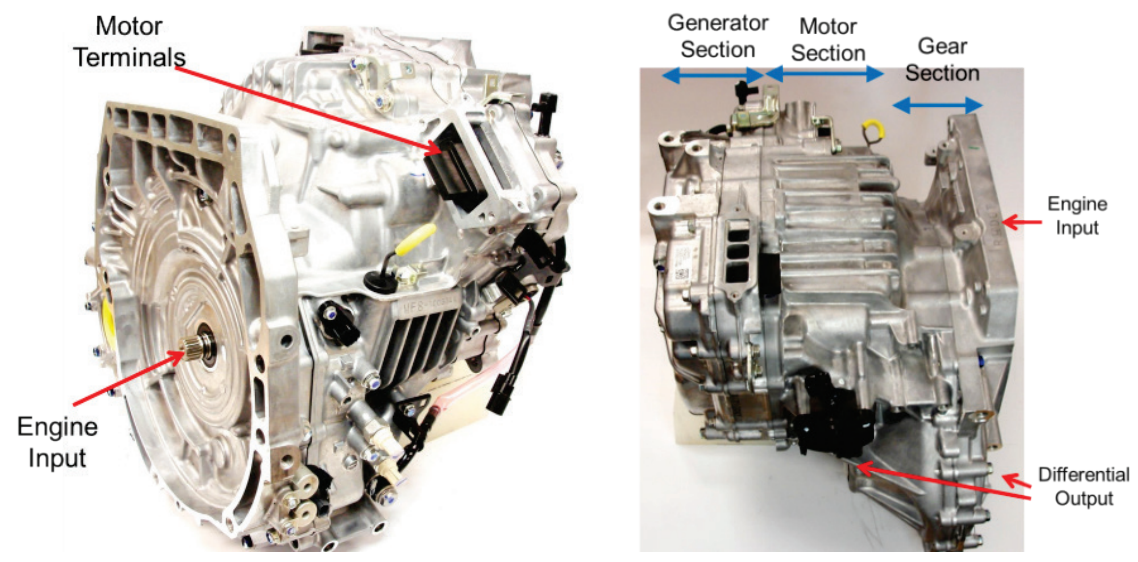

Figure 7: 2014 Honda Accord electronic continuously variable transmission.

After the end plate of the generator section is removed, the generator is clearly visible, as shown at left in Figure 8 . The generator end plate includes a position resolver that has 14 stator teeth, and the generator shaft has a soft-magnetic resolver rotor with 4 smooth lobes consisting of 8 laminations for position sensing. Also indicated at left in Figure 8 are four tubes used for oil spray cooling of the generator and motor end windings. Four groups of two holes are positioned in the tubes so that they spray the end turns on both sides of the generator and motor. Since the generator is smaller than the motor, the two groupings for the generator are closer together than the grouping of the motor spray holes. After the generator is removed, a clutch located between the generator and the motor is visible. The clutch does not have hydraulic activation and is used only as a torque limiter, most likely to mitigate transients and peak conditions when the generator is used to start the engine.
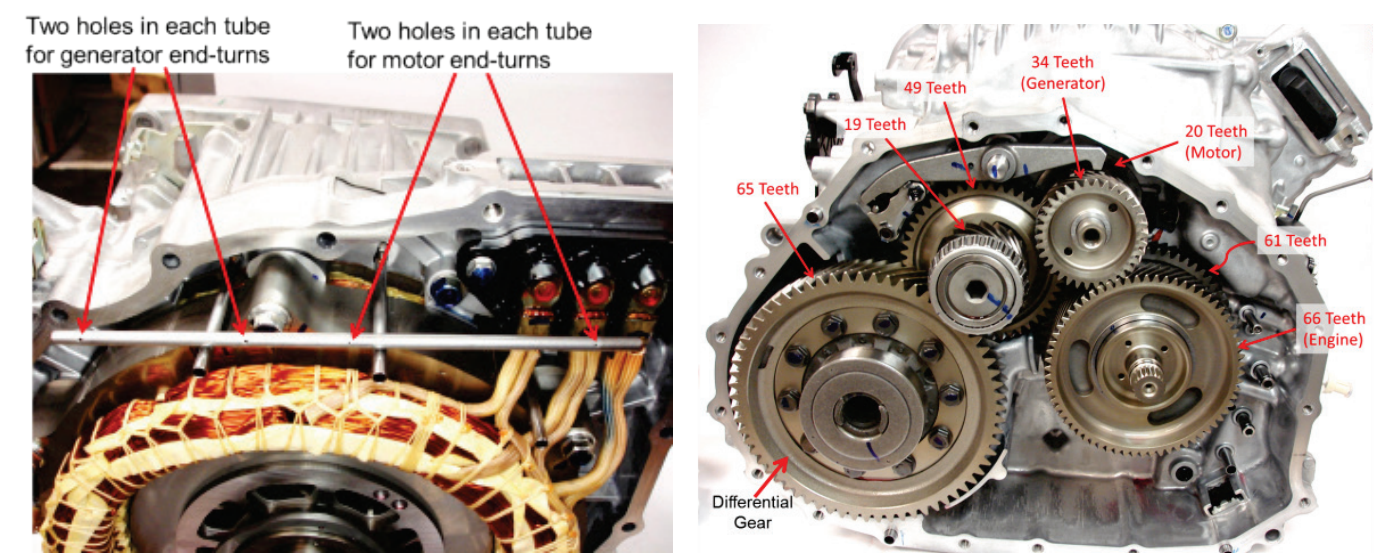

Figure 8: 2014 Honda Accord motor cooling system (left) and gear arrangement (right).

One side of the torque-limiting clutch is connected to the generator, and the other side is connected to a long shaft that feeds through the hollow motor rotor shaft and ultimately into the gear section of the transmission. At the end of the long generator clutch shaft, a small helical gear mates with a gear that is fixed to the splined engine input shaft, shown at right in Figure 8. Additional components in the gear section include a large differential gear, a drive gear, an overdrive clutch, and a small motor gear. The overdrive clutch is located between the engine input spline and the gear with 61 teeth that mates to the drive train.

During normal operation, the Accord hybrid system operates as a series hybrid, in which power from the engine is absorbed by the generator and passed to the battery and the electric motor for vehicle propulsion. However, at high speeds, the overdrive clutch engages the engine to the drive wheels through a fixed gear ratio. The number of gear teeth on each gear is indicated at right in Figure 8. The differential gear has 65 teeth, 
the driven gear has 39 teeth, the drive gear has 49 teeth, the clutched generator shaft has 34 teeth, the electric motor output shaft has 20 teeth, the clutched engine output has 61 teeth, and the engine input gear that mates with the generator gear has 66 teeth. Neglecting occasional slippage in the torque-limiting clutch, the generator rotational speed is 1.94 times faster than that of the engine.

Based on the original equipment manufacturer (OEM) tire size, the differential axle rpm is about 13 times the vehicle speed in miles per hour ( $\mathrm{mph}$ ). The total gear ratio from the electric motor to the differential output is 8.38; therefore, the rated motor speed of $14,000 \mathrm{rpm}$ correlates with a vehicle speed of about $128 \mathrm{mph}$. The common vehicle speed of $60 \mathrm{mph}$ correlates with an electric motor speed of about $6,500 \mathrm{rpm}$. When the overdrive clutch is active, the gear ratio from the engine to the differential output is about 2.75 , so engine speeds of 2,000 and 4,000 rpm correlate with vehicle speeds of 66 and $112 \mathrm{mph}$, respectively.

Stator and rotor laminations from the Accord motor and generator appear to be identical - the stator outer diameters are $29.13 \mathrm{~cm}$ (11.469 in.), the rotor outer diameters are $19.502 \mathrm{~cm}(\sim 7.678 \mathrm{in}$.), and the air gap is about $0.79 \mathrm{~mm}(0.031 \mathrm{in}$.). The motor stator stack length is $6.17 \mathrm{~cm}$, which is $\sim 1.64$ times the generator stator stack length of $3.762 \mathrm{~cm}$. The motor stator and rotor masses are 20.8 and $11.8 \mathrm{~kg}$, respectively, versus the generator stator and rotor masses of 14.4 and $8.3 \mathrm{~kg}$, respectively. The overall NdFeB magnet mass is $0.76 \mathrm{~kg}$ for the generator rotor and 1.24 for the motor rotor. Both stators (Figure 15) have 48 slots with 8 poles with windings comprising wires that are $18 \mathrm{AWG}$ in size. The motor has 20 wires in parallel for each phase; these split into 2 parallel paths, each having 4 poles in series before they combine at the neutral point. The generator motor has 22 wires in parallel for each phase. A comparison of a 2010 Toyota Prius rotor lamination and the 2014 Accord rotor lamination is provided in Figure 9. Although the designs are quite similar, two noticeably different features in the Accord lamination are a large oval-shaped hole between the "V"-oriented magnets and a radial slit extending outward radially from the oval.
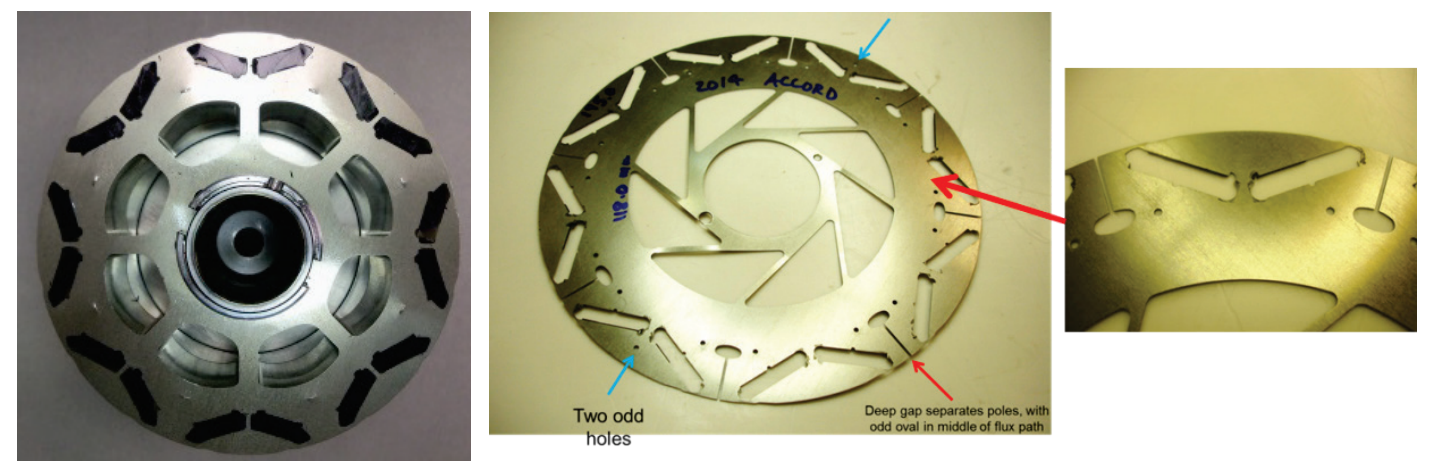

Figure 9: 2010 Prius rotor (left) and 2014 Honda Accord rotor lamination (right).

Electromagnetic finite element analysis (FEA) simulations were conducted to determine if the oval and slit features contribute any significant impact on the electromagnetic and torque characteristics of the motor. A flux density plot of the Accord simulation is shown at left in Figure 10, and a differential plot of FEA simulations with and without these features is shown at the right. There is very little difference in the stator flux density when these features are added, and notable differences only at and near the features. The simulated locked rotor torque versus electrical position with and without these features (labeled "With Cut" and "Without Cut") is shown at left in Figure 11. There is no significant difference when overall torque production and reluctance torque contribution are compared. Similarly, a comparison of running torque at right in Figure 11 indicates that the features have no significant impacts on torque ripple.

Building on previous collaborative efforts, ORNL coordinated with Argonne National Laboratory (ANL) to conduct background work and visited ANL's test facility to help with instrumentation and observation of component signals during full-vehicle testing of the 2014 Accord. Approximated motor torque values were recorded from the OEM CAN communication bus. Throughout extensive testing of the vehicle, CAN motor torque and power readings reached a maximum of $265 \mathrm{Nm}$ and $118 \mathrm{~kW}$, respectively. Measurements of various parameters during vehicle operation provided helpful feedback before detailed dynamometer analysis at ORNL. Examples of information obtainable via ANL vehicle testing are frequent dc link voltages, voltage relationship with battery state of charge, converter switching frequencies, and coolant temperature. Shown at left in Figure 12 are voltage and current waveforms measured during vehicle testing during operation at $5 \mathrm{mph}$. 
A fast Fourier transform of the voltage waveform indicates a fairly high-frequency distribution, whereas operation at almost all higher speeds resulted in primary components near and at integer multiples of $5 \mathrm{kHz}$. The latter is likely an indication of acoustic noise mitigation due to the use of higher-frequency pulse width modulation for low vehicle speeds and low acceleration levels.
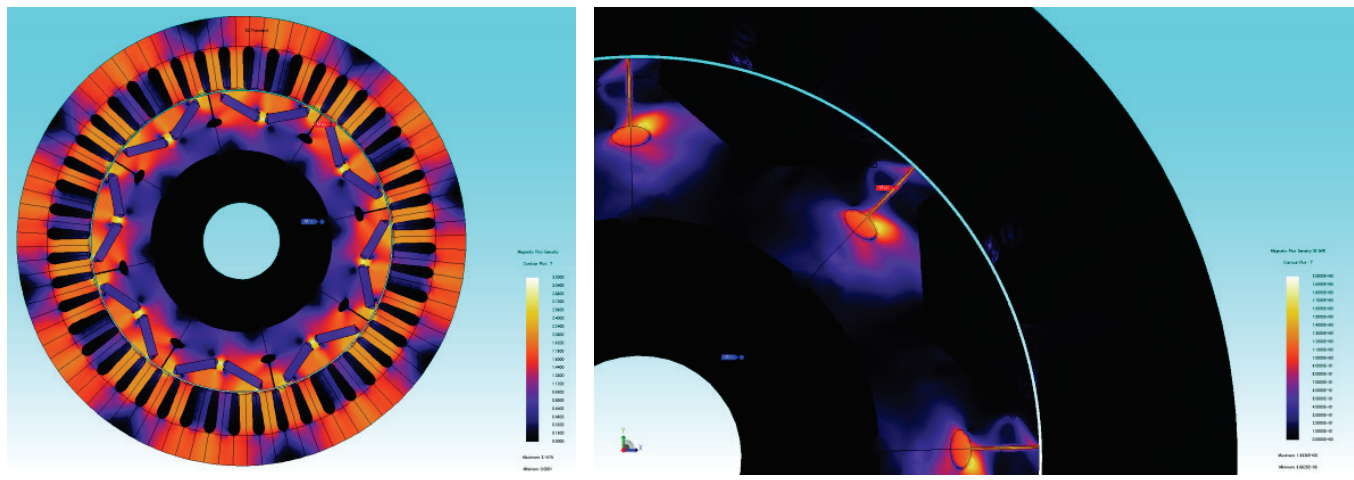

Figure 10: Flux density plot for 2014 Honda Accord (left) and differential plot of flux density — cut versus no cut (right).
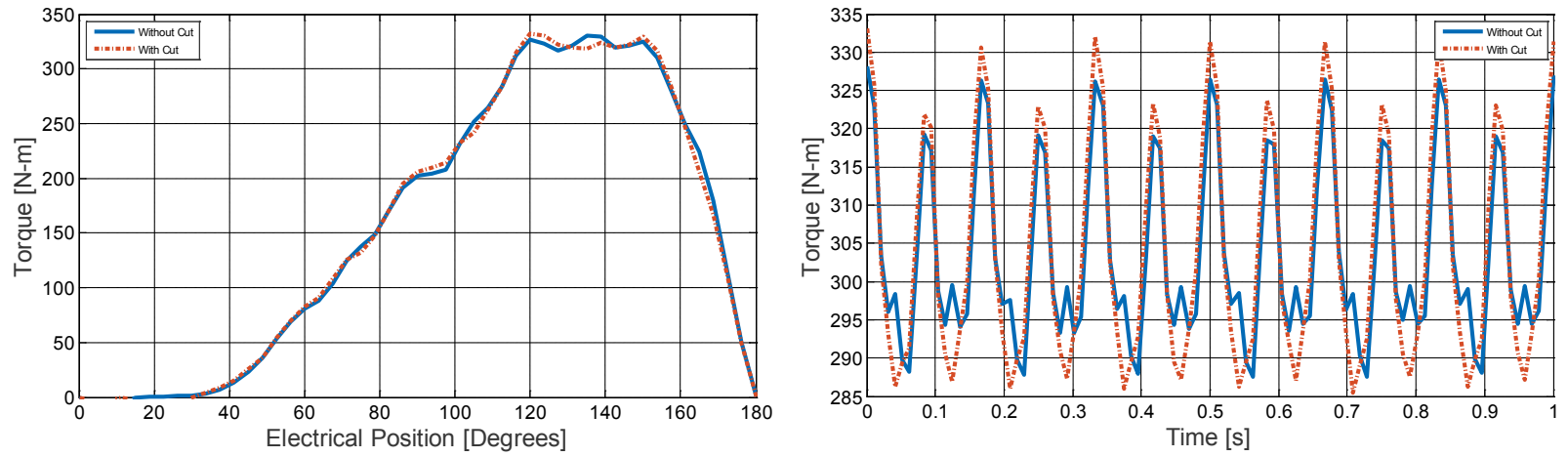

Figure 11: Simulated locked rotor torque (left) and running torque ripple (right).
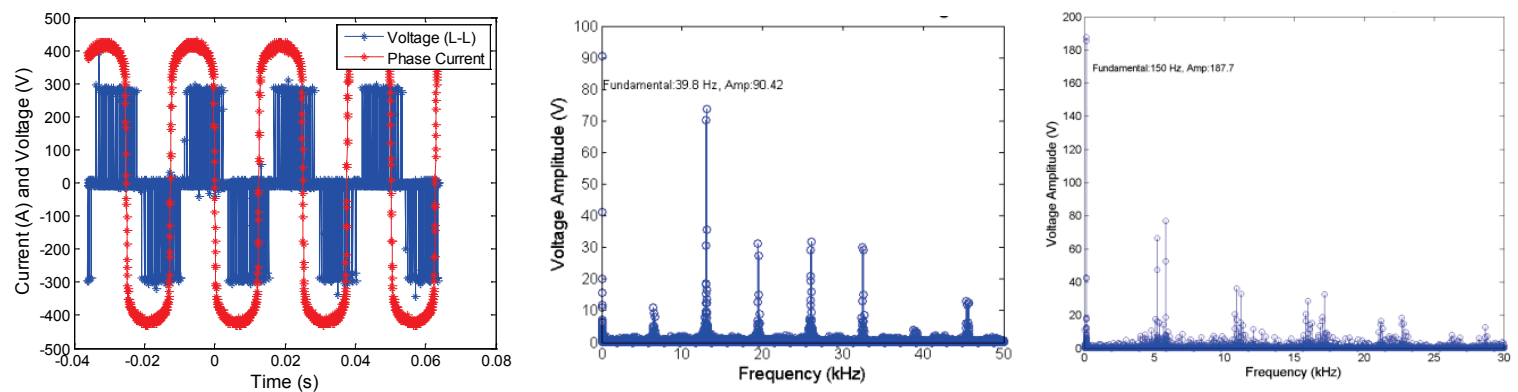

Figure 12: 2014 Accord running waveforms collected from vehicle in collaboration with ANL.

For detailed component testing at ORNL, designs were developed to provide access to the electric motor shaft in the transmission while maintaining the cooling and lubrication functionality used in the original form. This integration requires special attention to detail because high speed and power levels are involved. The electric motor shaft is not normally externally accessible, and unique methods were required to obtain access for dynamometer testing. A custom plate was designed with high tolerance alignment features and considerations for lubrication. A unique adapter shaft also was designed to adapt the motor shaft to ORNL's dynamometer, as shown in Figure 13. The graph at left in Figure 13 shows a comparison of back-electromotive force (EMF) results with those for other HEV motors. Locked rotor torque measurements for various positions and applied current are indicated at left in Figure 14. Test results indicate that a peak current of $\sim 425 \mathrm{~A} \mathrm{dc}$ is required to produce the published peak torque of $307 \mathrm{Nm}$. It was also observed that a significant amount of reluctance torque is produced in the motor. Additionally, the current angle at which the maximum torque occurs is 
slightly larger than that in most interior permanent magnet motor designs tested previously. For torques above $\sim 200 \mathrm{Nm}$, magnetic saturation begins to occur and the torque vs. current ratio drops from $\sim 0.85$ to 0.72 at peak torque, an indication that magnetic saturation is not extreme at peak torque.

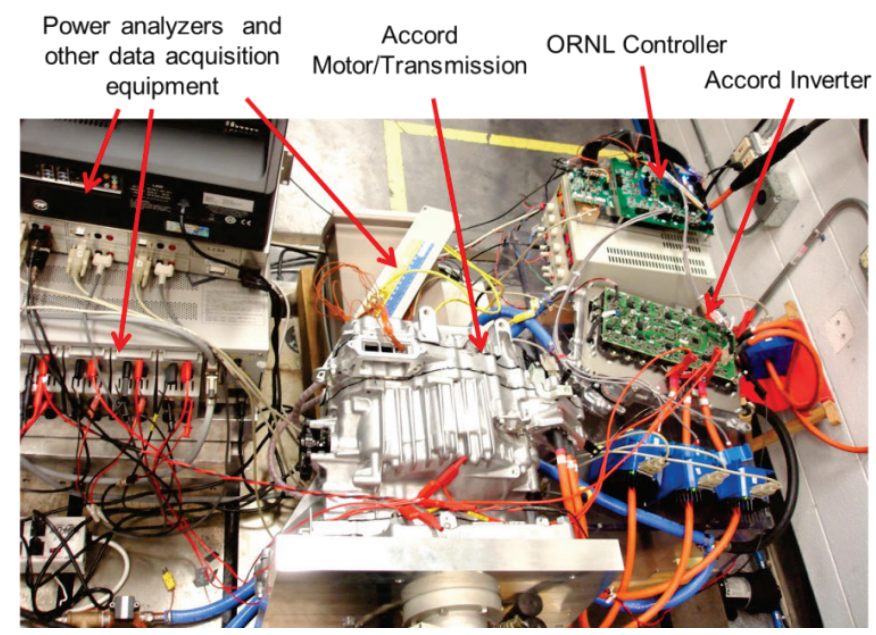

Figure 13: 2014 Honda Accord PCU and electronic continuously variable transmission components in ORNL dynamometer test cell.
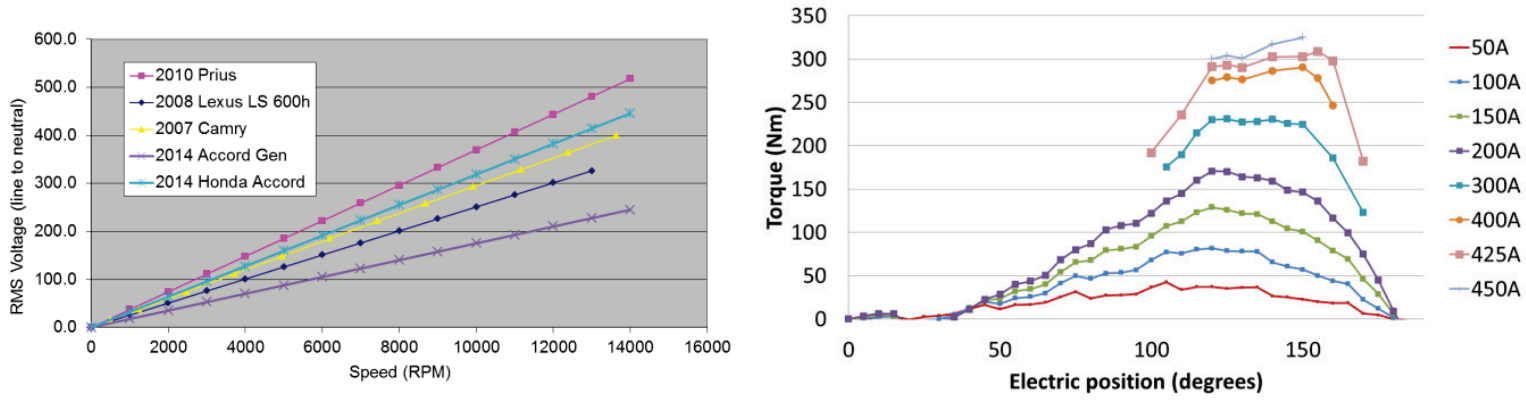

Figure 14: 2014 Accord motor back-EMF voltage (left) and locked rotor torque (right).

Performance testing and efficiency mapping for the 2014 Honda Accord was conducted at various torques and speeds for dc link voltage levels of 300, 500, and $700 \mathrm{Vdc}$. During these tests, the Accord inverter and motor were operated together, as optimal operation at each point was ensured. The inverter was cooled directly with standard automotive $50 \%$ water $/ 50 \%$ ethylene glycol coolant flowing at a rate of $10 \mathrm{~L} / \mathrm{min}$ with an inlet temperature of $65^{\circ} \mathrm{C}$. The motor and transaxle were cooled using the OEM oil cooling system, and the oil was cooled with a oil-water heat exchanger system that emulates typical radiator/oil-cooler functionality. Motor efficiency contours for operation at $300 \mathrm{Vdc}$ are shown in Figure 15. Motor efficiencies exceeding 93\% were observed between $\sim 2,200$ and 4,100 rpm and $\sim 60$ to $90 \mathrm{Nm}$. Inverter efficiency contours for the $300 \mathrm{~V}$ tests are shown in Figure 16, which shows efficiencies ranging from about 89 to $99 \%$ as speed increases. A combined efficiency map for both motor and inverter is shown in Figure 17. Efficiencies exceed $90 \%$ when the motor operates above torque levels of $\sim 50 \mathrm{Nm}$ at speeds greater than 2,000 rpm.

An efficiency contour map including motor and inverter (combined) efficiency for operation at $500 \mathrm{~V}$ is shown in Figure 18. This increase in voltage yields a considerable increase in the operation range, with an efficiency of $92 \%$ observed for a larger portion of the operating range. The peak region ranges from 4,000 to $9,000 \mathrm{rpm}$, between torque levels of $\sim 60$ and $160 \mathrm{Nm}$. For permanent magnet motors, voltage limitations are reached during high-speed operation because the back-EMF voltage induced by the permanent magnets increases with speed. Operation beyond these limits requires the application of field-weakening current, which yields some reluctance torque but ultimately leads to low-efficiency operation. Increased voltages allow for the motor to operate at higher speeds with relatively lower field-weakening current. 


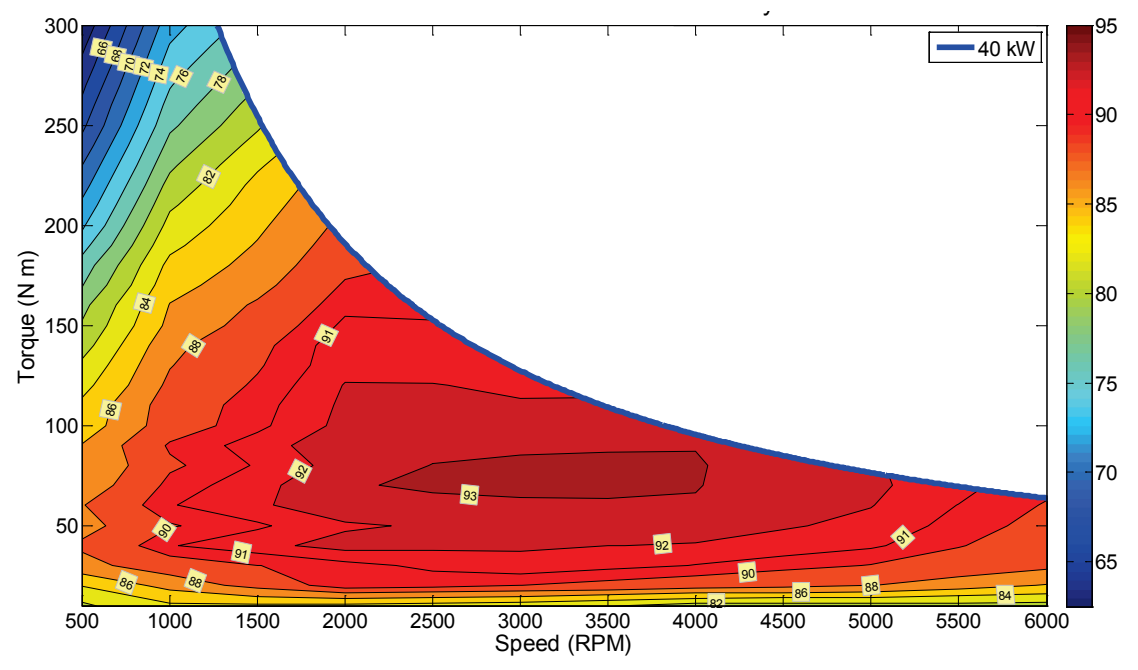

Figure 15: 2014 Honda Accord motor efficiency map at 300 Vdc.

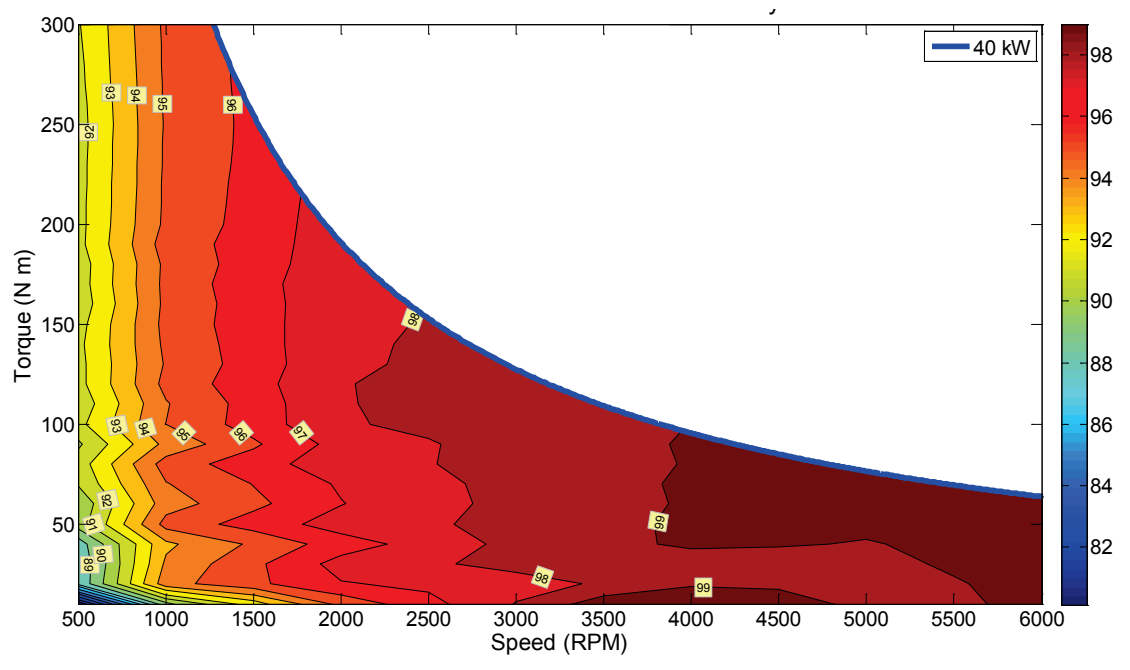

Figure 16: 2014 Honda Accord inverter efficiency map at 300 Vdc.

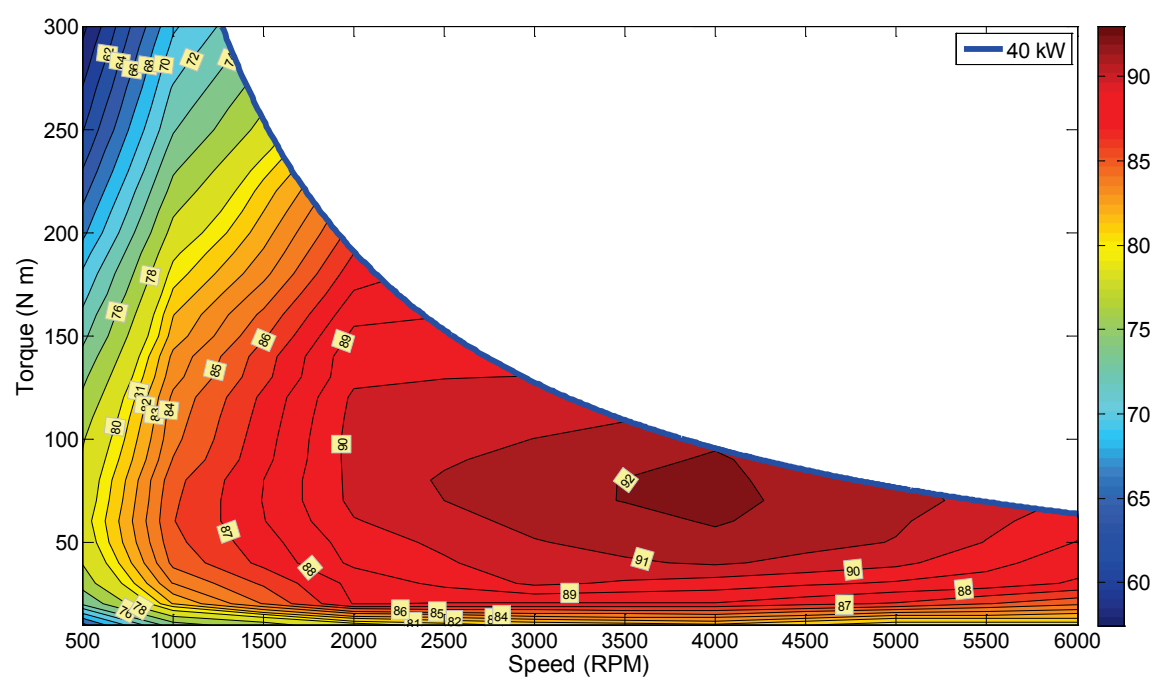

Figure 17: 2014 Honda Accord combined (motor and inverter) efficiency map at 300 Vdc. 


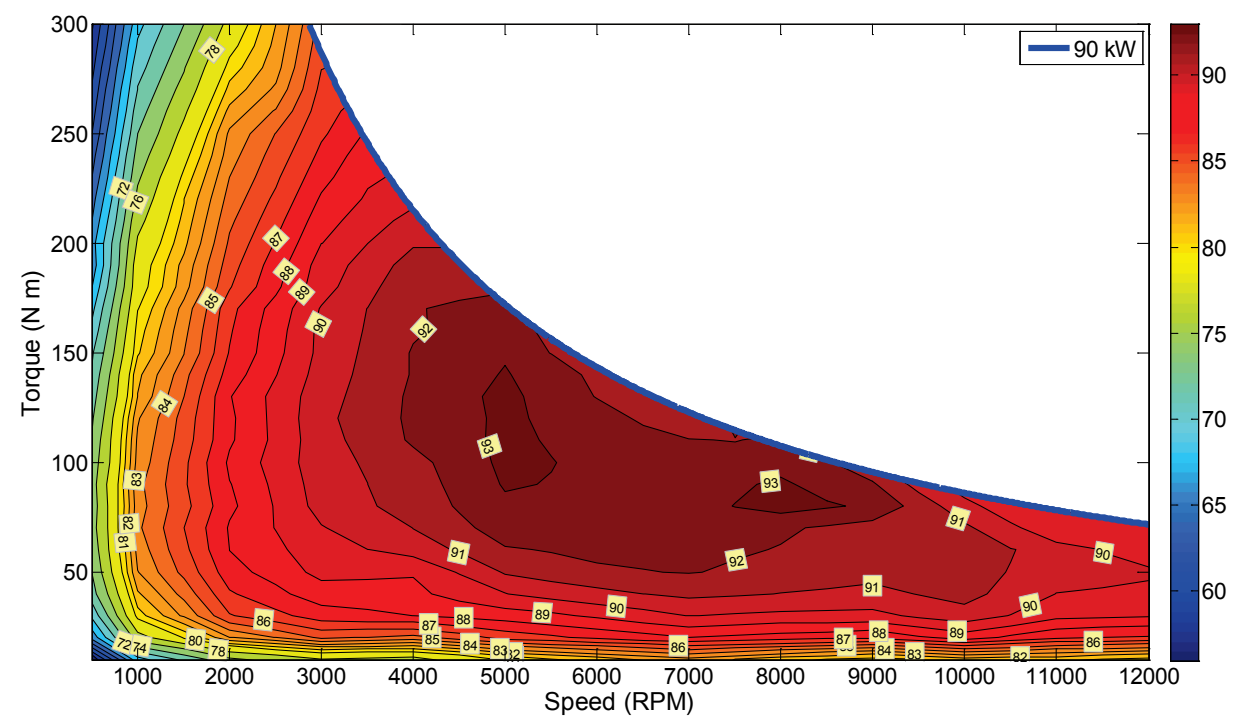

Figure 18: 2014 Honda Accord combined (motor and inverter) efficiency contours at 500 Vdc.

A motor efficiency contour map for operation at $700 \mathrm{Vdc}$ is shown in Figure 19. Motor efficiencies reached above $94 \%$ for speeds between $\sim 6,000$ and $10,000 \mathrm{rpm}$ and between torque levels of $\sim 40$ and $150 \mathrm{Nm}$. Inverter efficiencies for $700 \mathrm{Vdc}$ operation are shown in Figure 20, in which a peak inverter efficiency above $99 \%$ was observed for a considerable portion of the high-speed operation region. A combined (inverter and motor) efficiency contour map is shown in Figure 21, where the peak motor-inverter efficiency is $94 \%$ and efficiencies above $90 \%$ are noticeable for speeds ranging from about 3,500 to $12,000 \mathrm{rpm}$. During these tests, the published rated torque of $307 \mathrm{Nm}$ was confirmed up to a speed of 3,500 rpm. Tests also confirmed that the motor is capable of producing the published peak power of $124 \mathrm{~kW}$ at speeds between $\sim 4,000$ and $\sim 9,000 \mathrm{rpm}$.

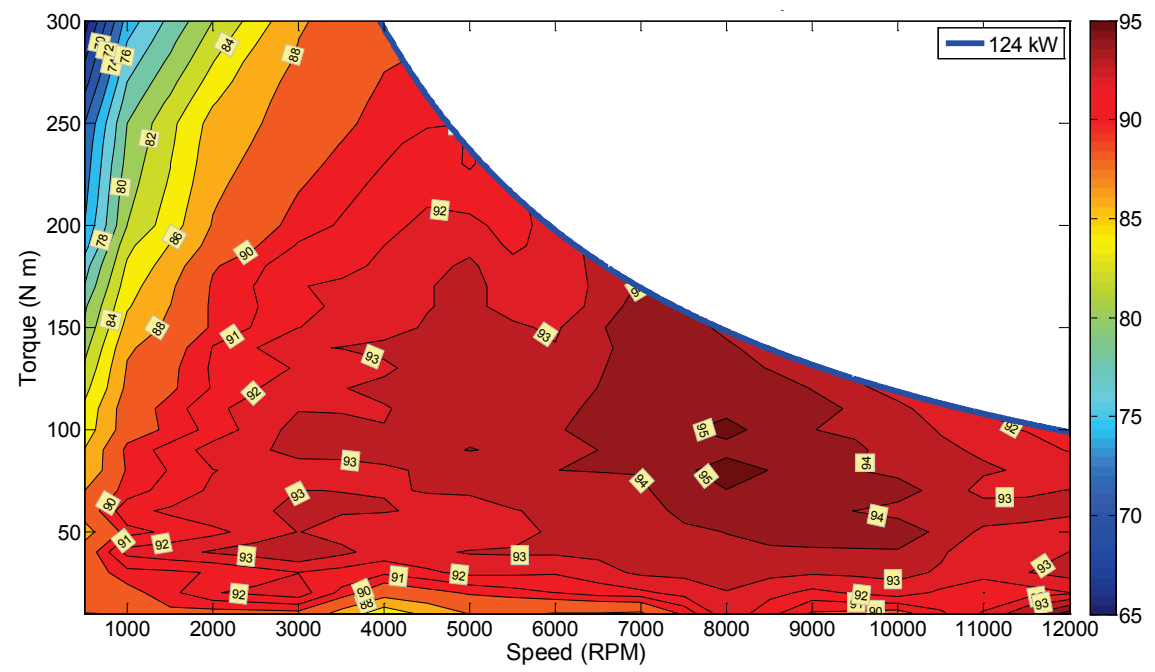

Figure 19: 2014 Honda Accord combined motor efficiency contours with 700 Vdc. 


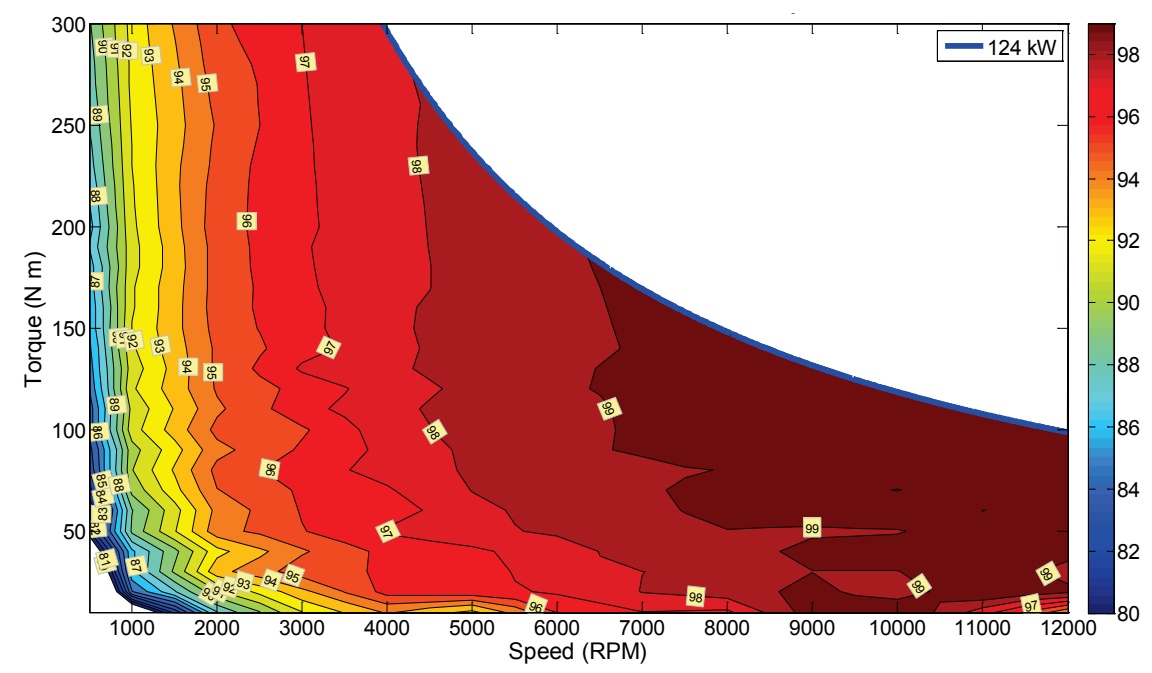

Figure 20: 2014 Honda Accord inverter efficiency contours with 700 Vdc.

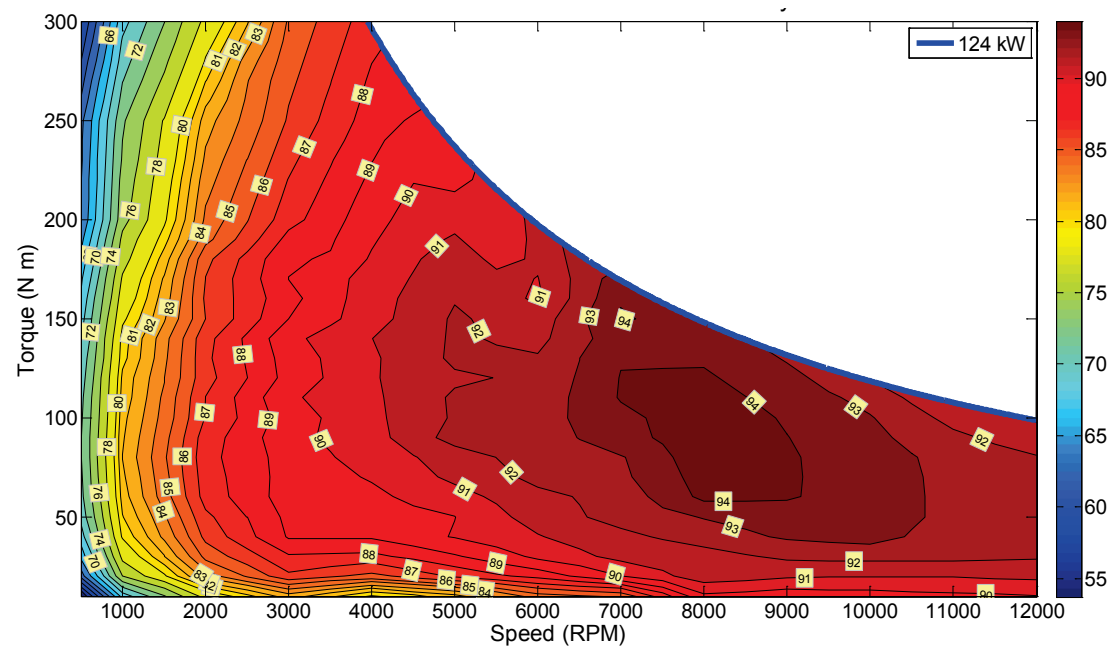

Figure 21: 2014 Honda Accord combined (motor and inverter) efficiency contours with 700 Vdc.

Continuous tests were conducted at 5,000, 7,000, and 9,000 rpm at power levels of 25 and $50 \mathrm{~kW}$. The plot in Figure 22 indicates the power level and motor winding temperature versus time for continuous tests at $7,000 \mathrm{rpm}$ and 25 and $50 \mathrm{~kW}$, with a dc bus voltage level of $700 \mathrm{~V}$. After operation at $25 \mathrm{~kW}$ for an hour, the motor temperature exceeded $100^{\circ} \mathrm{C}$. Then the power was increased to $50 \mathrm{~kW}$ for an hour, and the motor temperature was relatively stable at $\sim 135^{\circ} \mathrm{C}$. Note that this speed is associated with the highest efficiency at which $124 \mathrm{~kW}$ can be reached, and $124 \mathrm{~kW}$ operation at other speeds resulted in higher motor temperatures. 


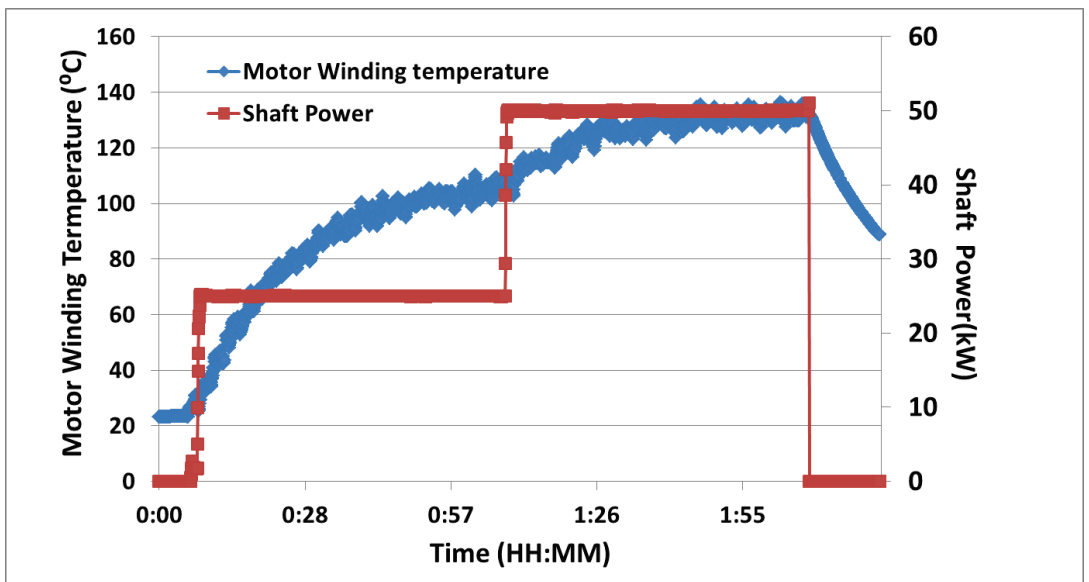

Figure 22: Continuous tests of 2014 Honda Accord at 7000 rpm and 25 and 50 kW.

\section{Conclusions and Future Direction}

Detailed disassembly and analysis of a PCU/inverter revealed key design features. They include a heat sink integrated with the power module, whereas most previously benchmarked components have used a thermal paste, which greatly inhibits heat transfer. In regard to cost, manufacturability, and power density, the inverter heat sink, bus bars, and other peripherals seemed slightly more complex than similar components observed from Toyota. Silicon nitride insulators were used, as opposed to the less expensive and more commonly used aluminum nitride. The inverter performed well thermally during peak and continuous power testing and the operation requirements.

Transaxle/electric motor teardown analyses yielded many interesting design features, including a comprehensive oil-spray cooling system that targets the end-windings of the motor and generator. Rotor laminations looked relatively similar to those of many Toyota products, except for a large gap located between the magnetic poles. Electromagnetic FEA simulations indicated that this feature has no significant impact on motor operation, but a benefit is gained with respect to mechanical stress relief. This facilitates a reduction in the thickness of the magnet supports near the airgap, thereby reducing leakage and increasing efficiency.

Operation at published peak torque and peak power was confirmed, and tests were conducted at 300,500, and $700 \mathrm{Vdc}$. Peak motor efficiencies reached $95 \%$ and inverter-motor efficiencies reached $94 \%$ at $700 \mathrm{Vdc}$ operation. Continuous tests indicated that the system is capable of operating continuously at $50 \mathrm{~kW}$, and the motor temperature remained below $140^{\circ} \mathrm{C}$.

Components for the BMW i3 were procured and will be the focus of comprehensive benchmarking studies in FY 2016. Additionally, there is high interest in benchmarking a state-of-the-art onboard charger, and efforts to procure a suitable candidate will continue.

\section{FY 2015 Presentations/Publications/Patents}

1. T. Burress, et. al, "Benchmarking of EVs and HEVs," presented at the DOE Vehicle Technologies Program Electric Drive Technologies FY15 Kickoff Meeting, Oak Ridge, Tennessee, November 18, 2014.

2. T. Burress, et. al, "Benchmarking EV and HEV Technologies: 2014 Honda Accord PCU," presented at the DOE Vehicle Technologies Program Electric Drive Technologies Electrical and Electronics Technical Team meeting, location, February 24, 2015.

3. T. Burress, et. al, "Benchmarking EV and HEV Technologies," presented at the U.S. DOE VTO 2015 Annual Merit Review, Washington DC, June 2015. 


\subsection{Power Electronics Packaging}

Zhenxian Liang, Principal Investigator

Oak Ridge National Laboratory (ORNL)

National Transportation Research Center

2360 Cherahala Boulevard

Knoxville, TN 37932

Phone: (865) 946-1467

E-mail: liangz@ornl.gov

Susan A. Rogers, DOE EDT Program Manager

Phone: (202) 586-8997

E-mail: Susan.Rogers@ee.doe.gov

Burak Ozpineci, ORNL EDT Program Manager

Phone: (865) 946-1329

E-mail: burak@ornl.gov

Contractor: UT-Battelle, LLC, managing and operating contractor for the Oak Ridge National Laboratory Contract No.: DE-AC05-00OR22725

\section{Abstract/Executive Summary}

- The project focused on the implementation of advanced packaging technologies in wide bandgap (WBG) semiconductor power modules to accelerate the application of WBG power semiconductor devices in the automotive industry. Most development work on packaging technologies conducted in FY 2015 is for silicon carbide (SiC) modules.

- Packaging technologies were designed and developed for the in-house manufacture of the SiC power modules for air-cooled inverters, using the latest industrial SiC devices and integrating electrical, thermal, and mechanical functions in a high-density package.

- An innovative integrated cooling package was developed based on planar SiC power modules. The inhouse-fabricated prototypes successfully demonstrated the integration of the electrical and cooling functions in power electronics building blocks. In addition, numerous simulations and experimental analyses were performed and validated the design features of this packaging technology, such as lower thermal resistance, small electric parasitic parameters, and efficient manufacturability.

- Working jointly with the material research projects (6.1, PE and EM Materials Support), silver (Ag) sintering bond technology was successfully used in the packaging for highly reliable SiC power modules. The packaging structure and associated packaging process technology were developed, and prototypes with different metal finishings on the substrate and different die layouts were manufactured for further evaluation.

\section{Accomplishments}

- Manufactured and delivered high-power SiC power modules for air-cooling system evaluation, which allow a reduction of $30 \%$ in overall volume and weight.

- Demonstrated that the integrated cooled planar SiC power electronics modules not only increase the power density by $60 \%$ but also enable a threefold increase in current density over their conventional silicon ( $\mathrm{Si}$ ) counterparts, resulting from a $35 \%$ reduction in the die size; 40 and $80 \%$ reductions in conduction and switching power losses, respectively; and a $35 \%$ reduction in package thermal resistance.

- Fabricated and delivered Ag-sintered die-attached $\mathrm{SiC}$ power modules. Initiated research on that technology to develop high-reliability, high-temperature WBG power electronics.

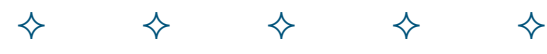




\section{Introduction}

State-of-the-art power inverters and converters in electric vehicles (EVs), as in the 2010 Toyota Prius and the 2011 Nissan LEAF, use Si power semiconductors and industrial drive-type packaging technologies; however, the electrical, thermal, and thermomechanical performance of these inverters is limited, as well as their manufacturability. These limitations cause large power losses, low semiconductor operational temperatures (limited to $150^{\circ} \mathrm{C}$ ), poor cooling $\left(0.6 \mathrm{~cm}^{2} \cdot{ }^{\circ} \mathrm{C} / \mathrm{W}\right)$, and poor power thermal/temperature cycling capabilities, resulting in a derating of the power devices. They also result in the need for costly semiconductors, as well as complicated manufacturing processes and overly bulky inverter/converter assemblies including an extra cooling loop.

WBG semiconductors such as $\mathrm{SiC}$ and gallium nitride $(\mathrm{GaN})$ permit devices to operate at much higher temperatures, currents/voltages, and frequencies - making power electronic modules using these materials significantly more powerful and energy efficient than those made from conventional semiconductor materials, such as Si. They also offer greater efficiency in converting electrical power and in operating the electric traction drive during vehicle use.

This research will lead to all-inclusive improvements in the performance and manufacturing of power modules for use in inverters/converters as a result of transitioning from Si to WBG power semiconductors and of innovations in packaging materials, structure, and processing. These comprehensive advances can directly affect the cost, efficiency, reliability, and density of power electronics systems in the electric drives of EVs. The goal of a $40 \%$ cost reduction and $60 \%$ power density increase in the power module supports DOE EDT 2022 power electronics targets of $\$ 3.3 / \mathrm{kW}$ and $14.1 \mathrm{~kW} / \mathrm{kg}$, respectively.

\section{Approach}

The project focuses on the design and development of advanced packaging technologies for all-SiC power modules, enabling the exploitation of SiC's superior performance. Three technologies - packaging of an aircooled inverter module, integrated cooling packaging of planar power modules, and Ag-sintering die bonding for high-reliability power modules - were investigated, as well as packaging structure and associated process technology. Performance improvements were determined through experimental measurements; and efficiency, cost, and reliability benefits to power electronics systems were evaluated.

\section{A. SiC Power Module Packaging for Air-Cooled Inverters}

A forced-air-cooled SiC inverter was developed as part of another EDT project (3.1, Inverter R\&D). The all$\mathrm{SiC}$ power module has a one-phase-leg configuration composed of $\mathrm{SiC}$ metal-oxide-semiconductor field-effect transistors (MOSFETs) and SiC Schottky barrier diodes (Figure 1), which is the basic building block for various automotive power converters and inverters. Power SiC MOSFETs and diodes are commercially available in the form of bare dies. The current rating of the power module can be multiplied by paralleling more dies. For the designed air-cooled power inverter, the switch units consist of three parallel bare dies of MOSFETs (each rated at $80 \mathrm{~A}, 1200 \mathrm{~V}$ ) and three bare dies of diodes (each is rated at $50 \mathrm{~A}, 1200 \mathrm{~V}$ ).

Figure 2 presents a schematic of this module's packaging structure in which the upper unit of a phase-leg inverter, including all U-MOSFETs and U-diode dies, is attached to a direct bond copper (DBC) substrate and interconnected through bond wires. In the same way, in the lower unit, L-MOSFETs plus L-diode dies are attached to another DBC substrate. These two power units are mounted mechanically onto the two sides of a custom-manufactured heat sink, with a thermal interface material applied between the substrates and heat sink. All power devices and interconnections are also encapsulated for mechanical protection and electrical insulation. 


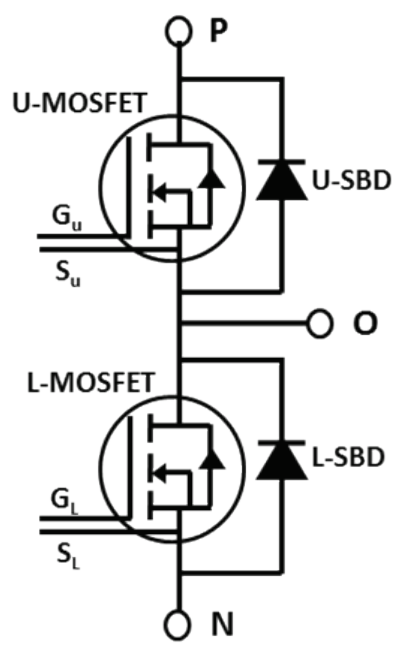

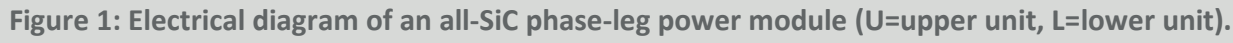

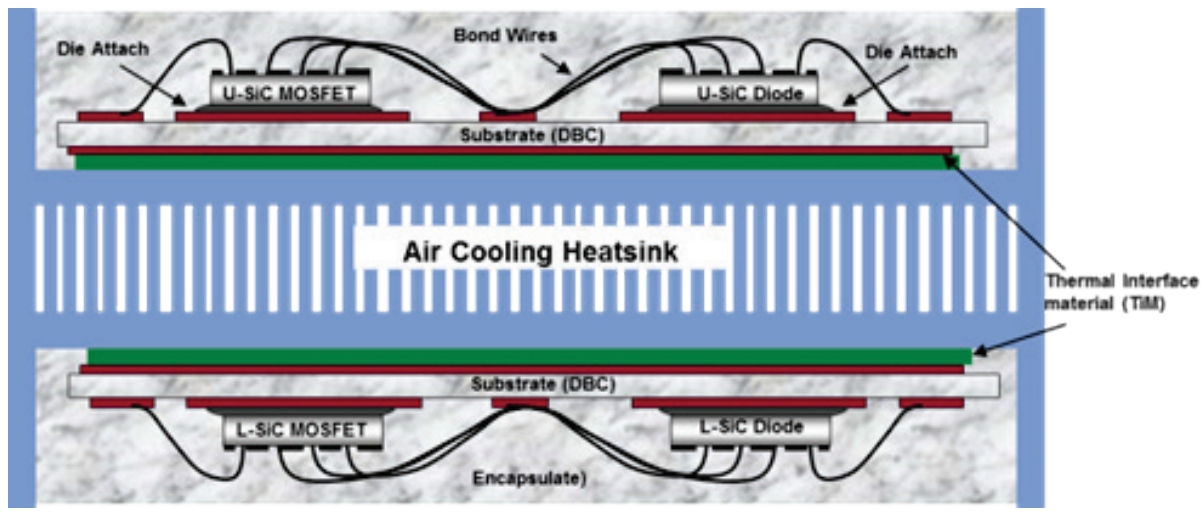

Figure 2: Schematic packaging structure of forced-air-cooled SiC phase-leg power module.

This module packaging design eliminates the baseplate and solder layer used in a standard packaging structure and effectively reduces the thermal resistance of the module. In addition, the integral heat sink helps increase the power density of the inverter.

\section{B. Integrated Double-Sided Cooling of SiC Power Modules}

State-of-the-art WBG discrete devices and multi-chip modules are manufactured using technologies that follow packaging schemes used for Si devices, employing well-established wire bond technology and a thermal stacking structure suitable for single-side cooling. In power converter/inverter assemblies, as shown in Figure 3, the module is attached on one side to a heat sink or cold plate providing liquid cooling for the devices in the modules. Through this packaging configuration, the fundamental packaging functions-e.g., electrical interconnection, thermal management, and mechanical support - are met. However, this hybrid package has limitations in its electrical performance, cooling capability, thermomechanical properties, and manufacturability. 


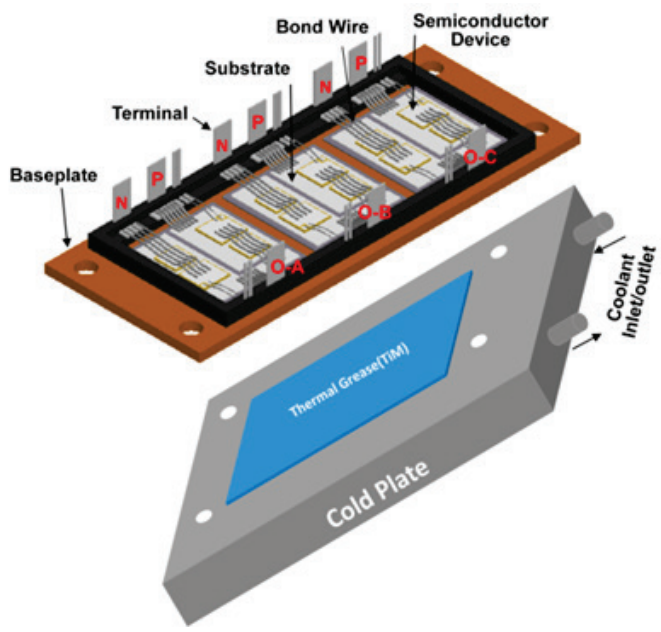

Figure 3: Schematic of conventional packaging of power electronics modules.

To overcome these limitations, an innovative packaging structure was proposed (Figure 4), in which multiple planar SiC power modules with pin-fin cold plates on both sides are embedded into a plastic manifold (in black), which guides the coolant going through the pins on both sides of the cold plates. The inlet and outlet are arranged on one side of the block, and the electrical inputs/outputs are on the other three sides. Thus, an integrated electronics building block, including both electrical interconnection and thermal management, will replace the power electronics assembly shown in Figure 3. Furthermore, direct, double-sided cooling ensures minimum thermal resistance in this package; and the 3-dimensional electrical interconnection in the planar module and compact connection in the inverter results in the lowest parasitic electrical parameters, which enable $\mathrm{SiC}$ 's superior performance in power electronics systems.

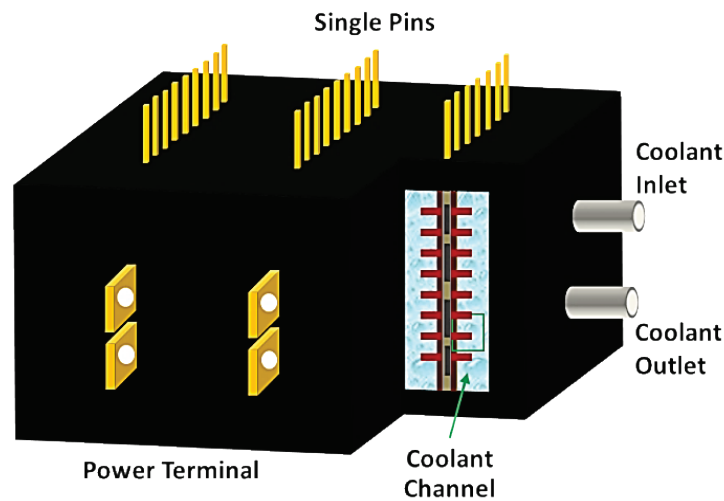

Figure 4: Schematic of an integrated cooling structure of multiple planar SiC modules for a multiphase converter/inverter.

\section{Planar-Bond-All SiC Power Module}

Figure 5 presents a cross-sectional view of the planar-bond-all (PBA) SiC power module with a phase-leg electrical configuration, as shown in Figure 1. The all-MOSFET and diode dies are sandwiched between two DBC substrates. The electrical interconnection is achieved by conductively bonding both the top and bottom sides of the dies to the copper traces on the two substrates, which are patterned to form circuitry corresponding to the electrode pad layout on the dies. The DBC substrates provide electrical insulation via the internal ceramics (such as aluminum nitride) between two copper layers. This symmetric planar-bonded package offers flexibility in the arrangement of the switch dies. As shown in Figure 5, the upper switch pair and lower switch pair in the phase-leg topology are oriented in a face-up/face-down configuration that significantly reduces the electrically parasitic inductance and resistance.

In addition, two pin-fin base plates made of copper are directly bonded to the power package from the back sides of both DBC substrates by another soldering process. Thus, after encapsulation of the plastics (black color), a direct and double-sided cold power module is ready for further integration. 


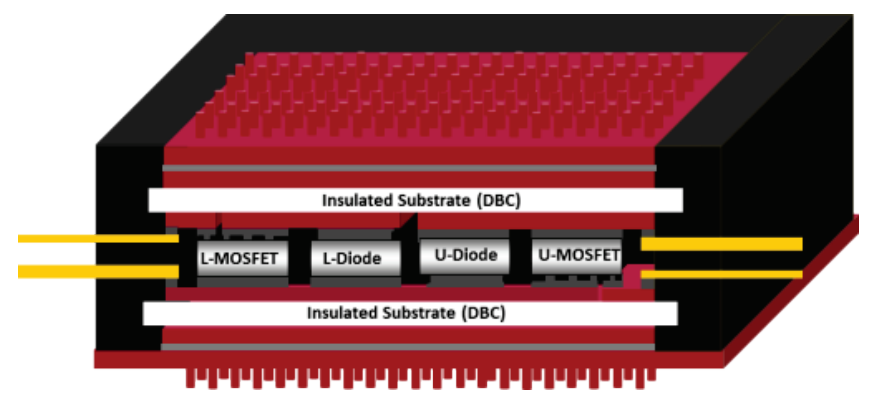

Figure 5: Cross-sectional view of a planar-bond-all SiC phase-leg power module.

To realize the concept shown in Figure 4, a special coolant manifold was assembled, as shown in Figure 6. The parts are made of plastic and manufactured using additive manufacturing (3D printing) technology. The manifold is designed to provide liquid passageways for coolant passing mainly through the pin-fin areas. The open tubes provide coolant inlets and outlets, and the inner channels serve to guide the coolant flow uniformly.

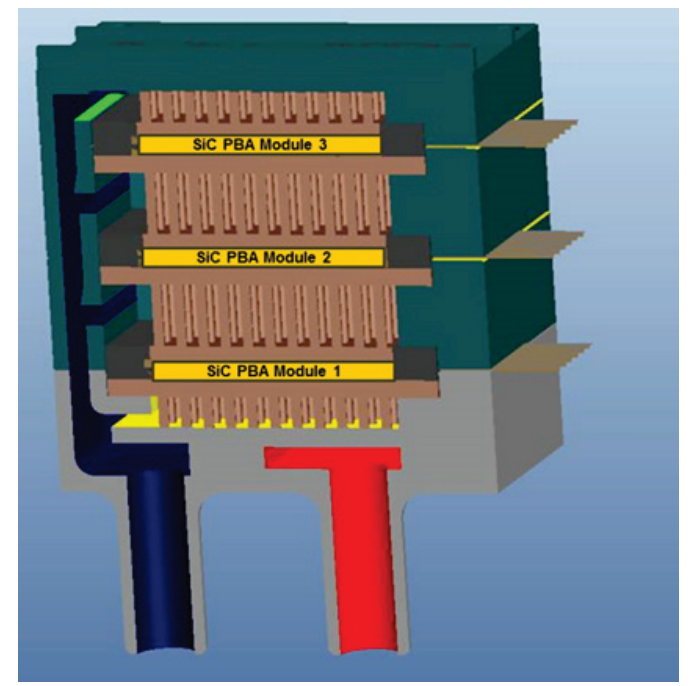

Figure 6: Design for assembly of an integrated double-sided, direct liquid-cooled SiC power electronics block.

\section{Ag-Sintered Die Attachment}

The Ag layer formed during the sintering process, from a paste mixed with nanoscale Ag particles and other chemicals, is known to be a superior die attachment material compared with the solder layer formed during the soldering process. It offers much better electrical, thermal, and especially thermomechanical properties for power module packaging. These properties are especially critical to $\mathrm{SiC}$ power devices, allowing them to operate at temperatures higher than those of Si devices. However, Ag sintering also includes an additional process of mechanically pressing the stack of dies and substrates during heating. The quality of the bond formed between die and substrate can be affected by many factors, such as finishing metals on the die and substrate, geometry of the die, and patterns on the substrate, as well as process parameters - pressure, heating temperature, and heating time. Based on fundamental studies using various coupons, research on Ag sintering of $\mathrm{SiC}$ device dies on DBC substrate began this year. Figure 7 shows schematically the packaging design of this $\mathrm{SiC}$ power module sample and where the $\mathrm{Ag}$-sintering process is employed to realize the die attachment, instead of soldering, as in conventional power modules. Wire bonds and gel encapsulation are also included in the fabrication of a complete power module for electrical thermal and thermomechanical tests. 


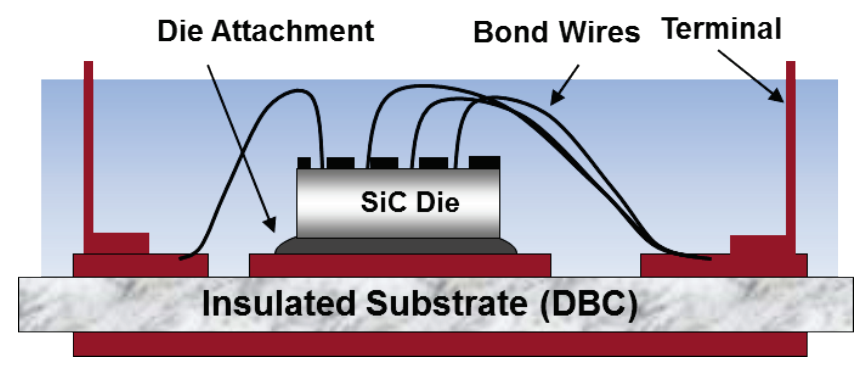

Figure 7: Cross-sectional view of an SiC die bonded onto a DBC substrate.

\section{Results and Discussion}

The development of innovative packaging technologies promotes the application and improves the performance of the latest WBG semiconductors in electric drive systems for the automotive industry.

\section{A. SiC Power Module for Air-Cooled Inverter}

A number of $\mathrm{SiC}$ power modules for air-cooled inverters have been successfully fabricated at ORNL. Figure 8 shows three groups of packaged $\mathrm{SiC}$ power units. As described in Figure 2, the power devices for the upper unit and lower unit in a phase-leg configuration (Figure 1) are packaged separately onto two DBC substrates. The left unit carries the lower devices (three MOSFETs and three diodes), and the right unit includes the upper devices (three MOSFETs and three diodes) in each group. The bare $\mathrm{SiC}$ dies used were the latest products released by industry vendors. The ratings of the MOSFET die are $80 \mathrm{~A}$ and $1200 \mathrm{~V}$, and the diodes are rated at $50 \mathrm{~A}$ and $1200 \mathrm{~V}$. To meet the designed power capability, three dies are packaged in parallel on the DBC substrates.

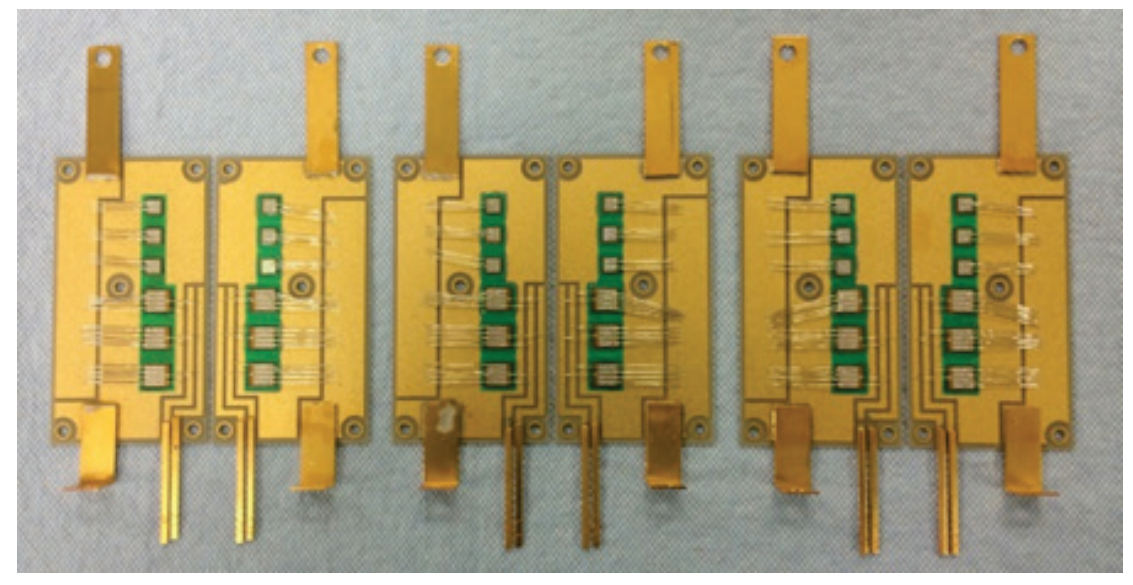

Figure 8: Three groups of packaged SiC power device units for air-cooled inverter.

By incorporating the heat sink design, the layouts on the DBC substrates have been designed for optimal electrical and thermal performance. The big power terminals $\mathrm{P}, \mathrm{O}$, and $\mathrm{N}$, as marked in Figure 1, are mounted on the DBC substrates close to the devices; whereas the gate drive signal pins $\mathrm{Gu}, \mathrm{Su}, \mathrm{G}_{\mathrm{L}}$, and $\mathrm{S}_{\mathrm{L}}$ are mounted on the separated traces, forming a Kelvin configuration and eliminating the interference from the main power loop when the devices are switching. All of these measures also minimize any electrical parasitic parameters (inductance and resistance).

To optimize the thermal management of the inverter, an aluminum nitride ceramic DBC was employed with a thermal conductivity of $180 \mathrm{~W} / \mathrm{m}^{\circ} \mathrm{K}$, which is much higher than that of a conventional aluminum oxide ceramic $\left(20 \mathrm{~W} / \mathrm{m}^{\circ} \mathrm{K}\right)$. Combined with $\mathrm{SiC}$ 's high thermal conductivity $\left(180 \mathrm{~W} / \mathrm{m} \cdot{ }^{\circ} \mathrm{K}\right)$, it efficiently transferred the heat generated in the devices to the bottoms of the modules. 
To fabricate these units, the first step was to attach all six dies, two power terminals, and two pins using a soldering process. A special jig was designed that made for the precise alignment of all the components. Solder preforms were used and placed beneath each component before one heating (solder reflow) profile. Then, multiple aluminum wires (10 mil in diameter) were bonded on the chips and substrate for electrical connection.

As shown in Figure 2, two packaged units were directly mounted onto a heat sink from both the top and bottom surfaces by mechanical pressing. To do so, five through holes were made in each DBC substrate. Figure 9 is a photo of the phase-leg $\mathrm{SiC}$ power module assembly with the lower unit visible. The heat sink was specially designed and manufactured. To improve thermal transfer, a thin thermal grease layer was applied beneath the DBC substrate to keep the substrate and heat sink in close contact. This simplified thermal stack ensured that the assembly had low thermal resistance.

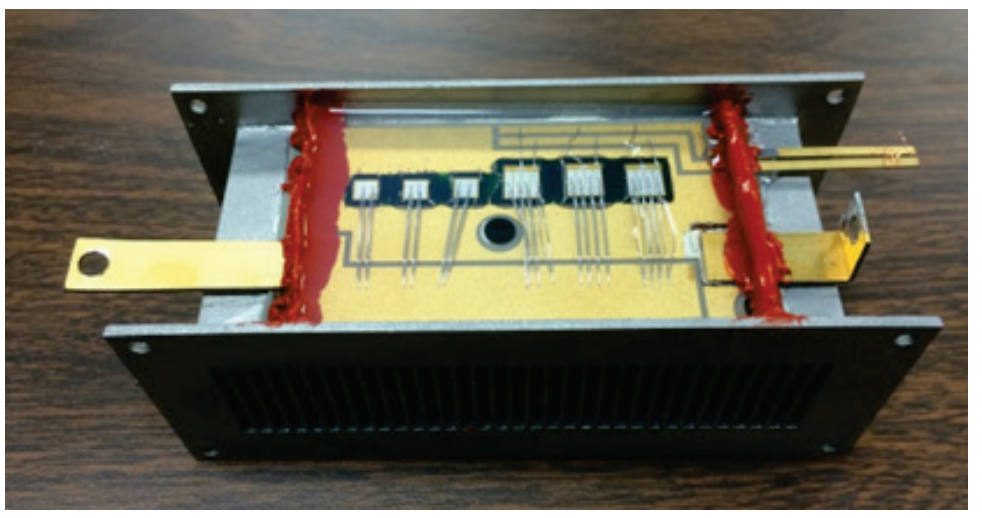

Figure 9: Packaged one-phase leg SiC power module assembled on a heat sink for an air-cooled inverter.

Potting encapsulation was used to provide electrical and environmental protection to the DBC substrate, the devices, and the bond wires.

The module assemblies were delivered to another EDT project (3.1, Inverter R\&D) to build and test a 3-phase air-cooled $\mathrm{SiC}$ inverter.

\section{B. Integrated Double-Sided Cooling of SiC Power Modules}

Fabrication of the PBA SiC power module has been described in previous reports. The key difference is that the top interconnections of the dies use a planar, larger-area bond to their top electrodes, instead of multiple tiny wire bonds. This change makes it possible to integrate many superior packaging concepts into power modules. Figure 10 shows photos of such a module fabricated in the ORNL packaging laboratory.

Figure 10(a) is the package of a $100 \mathrm{~A}, 1,200 \mathrm{~V} \mathrm{SiC}$ phase leg power with the topology illustrated in Figure 1. The power device upper or lower unit consists of two MOSFET dies and diode dies for power ratings at $100 \mathrm{~A}$, $1200 \mathrm{~V}$ (the rating of each die is $50 \mathrm{~A}, 1200 \mathrm{~V}$ ). All eight SiC dies were bonded between two DBC substrates by solder from both the top and bottom surfaces (see Figure 5). At the same time, the power terminals P, N (on the left side), and $\mathrm{O}$ (on the right side) and signal pins $\mathrm{Gu}, \mathrm{Su}, \mathrm{G}_{\mathrm{L}}$, and $\mathrm{S}_{\mathrm{L}}$ (on the upper side) were also mounted onto the DBCs. A soldering process was employed to form the planar bonds. It offers high electrical conductivity and easy processing. As shown at the top left, bond wires were used to connect the device electrodes and pads on the DBC. Again, the Kelvin configuration was used here to avoid interference between the gate drive loop and power loop. This module measures $40 \times 40 \times 2 \mathrm{~mm}$, excluding the terminals and pins.

Figure 10(b) shows the attachment of the dual cold plates from both sides, in which two pin-fin heat sinks made of copper were directly bonded onto the back sides of the DBC substrates by two solder layers (one is shown in photo (a), grey color) with a lower melting point. Another soldering process was employed using a special jig for these two-layer solder reflows. The area of each effective pin fin array is $38 \mathrm{~mm} \times 38 \mathrm{~mm}$; the fin height is $7 \mathrm{~mm}$. 


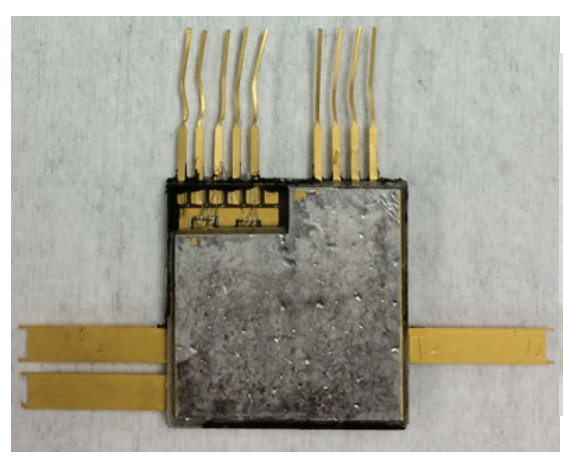

(a)

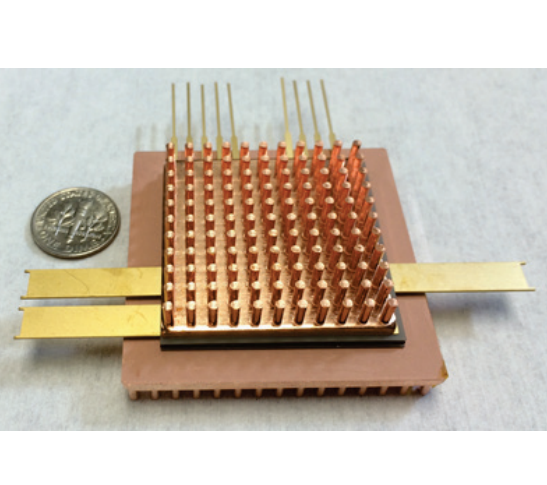

(b)

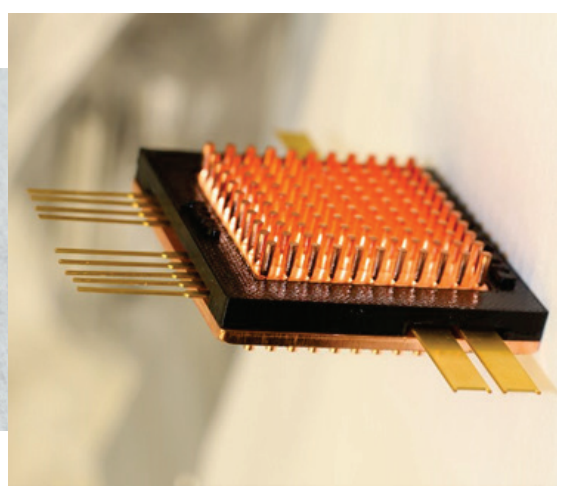

(c)

Figure 10: Photos of a 100 A, 1,200 V PBA SiC MOSFET/JBS diode phase-leg power module: (a) 3-dimensional planar interconnections, (b) double-sided cooling with two pin-fin substrates directly bonded on both sides, and (c) the final encapsulated module.

Figure 10(c) shows the final encapsulated module. A premade plastic frame was attached to the bottom pin-fin baseplate. Silicone gel or epoxy then filled the gaps and cavities using the potting process. The encapsulation provides electrical insulation for high-voltage $\mathrm{SiC}$ devices and strong mechanical support to ensure the integrity of the modules. The final package measures $50 \times 50 \times 8 \mathrm{~mm}$, excluding terminals and pins.

Following the design shown in Figure 6, integrated double-sided liquid cooling of multiple phase-leg inverters can be achieved by assembling them in a special coolant manifold. Figure 11 shows such an integrated power electronics building block. The parts of this special coolant manifold were made of plastic and manufactured by 3-D printing. The manifold was designed to match the form factors of the module discussed above, providing sealed liquid passageways for the coolant passing through mainly the pin-fin areas. The multi-part manifold was assembled seamlessly together with the modules using a sealing polymer and mechanical joining.

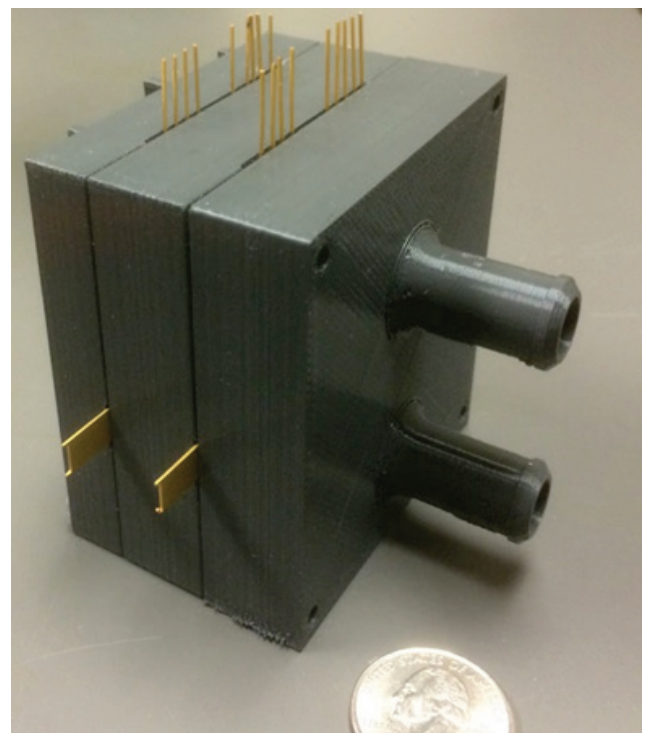

Figure 11: Double-sided integrated direct liquid cooling of PBA module: two phase-leg power modules in a coolant manifold.

The packaging of the semiconductor devices brings with it parasitic electrical components such as resistance, inductance, and capacitance, adding to SiC's intrinsic electric parameter network. These parasitic components not only consume electrical power but also increase the power losses of the $\mathrm{SiC}$ devices by limiting their operational capability, such as $\mathrm{dV} / \mathrm{dt}, \mathrm{dI} / \mathrm{dt}$, and blocking voltage, as well as electromagnetic compatibility.

Figure 12 shows electrical parasitic parameters in the PBA SiC module obtained using a simulation tool known as MAXWELL Q3D Extractor. This tool calculates the electromagnetic field and extracts the parasitic components through current conduction paths. Figure 12(a) shows a typical current density distribution in 
interconnection loops inside the package. The electrical components responsive to each section of the interconnection traces are shown in a lump-element circuit model of the entire phase-leg module. The components enclosed within the dashed line are related to the outside signal pins. This model provides a basis for further performance simulation of power converters and/or inverters built of these modules. The loop inductance from terminal $\mathrm{P}$, through upper MOSFETs and lower diodes to terminal $\mathrm{N}$, is usually considered to be a feature parasitic parameter at $11.9 \mathrm{nH}$ for the PBA SiC module.

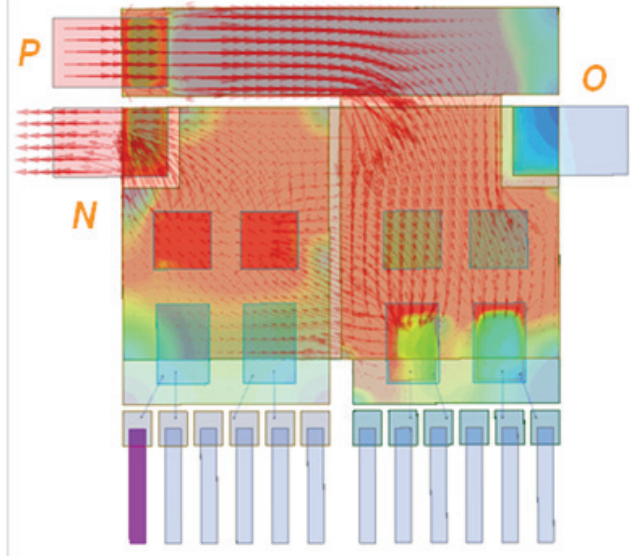

(a)

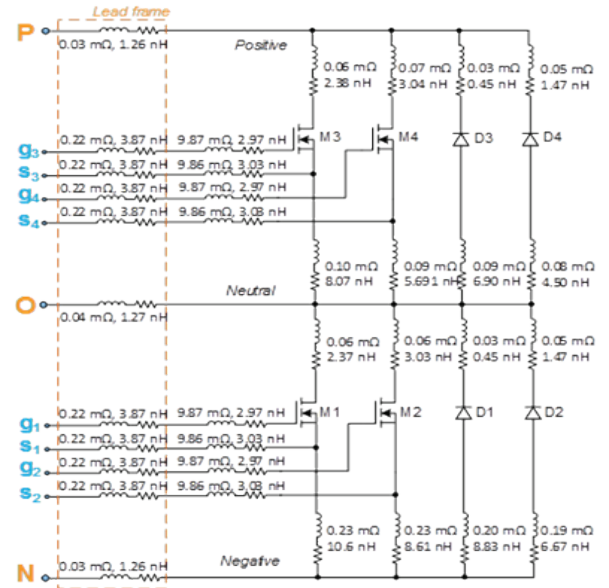

(b)

Figure 12: Extraction of electrical parasitic parameters in PBA SiC power module: (a) simulated current distribution and (b) lump element model.

The electrical performance of the PBA SiC power module was evaluated experimentally. Figure 13 shows an experimental setup in which the PBA module is very closely connected to a bus capacitor bank with negligible additional feature inductance. Compared with the benchmark inverter, the feature inductance can reach more than $100 \mathrm{nH}$. The PBA module cut the parasitic inductance by $80 \%$. On the other side of the setup, the gate drive circuitry is connected to the module at the shortest distance to the devices, eliminating the parasitic components of the outside pins, as shown in Figure 12(b). Those components would affect the gate drive signal fidelity, causing undesired voltage and current ringing and limiting the switching speed of SiC devices, resulting in a greatly derated regime. The significant reduction in these parasitic components makes it possible to operate the $\mathrm{SiC}$ devices at higher frequency and higher efficiency.

Figure 14 shows the voltage and current waveforms when the module is operated under switching conditions. A much smaller overshoot is added to a $600 \mathrm{~V}$ bus at the switching-off transition, indicating smaller feature inductance with the module. Lower inductance allows the MOSFETs in the PBA module to operate at a higher $\mathrm{dI} / \mathrm{dt}$, an important feature of $\mathrm{SiC}$ devices and an effective way to reduce the switching losses and increase the efficiency of $\mathrm{SiC}$ inverters.

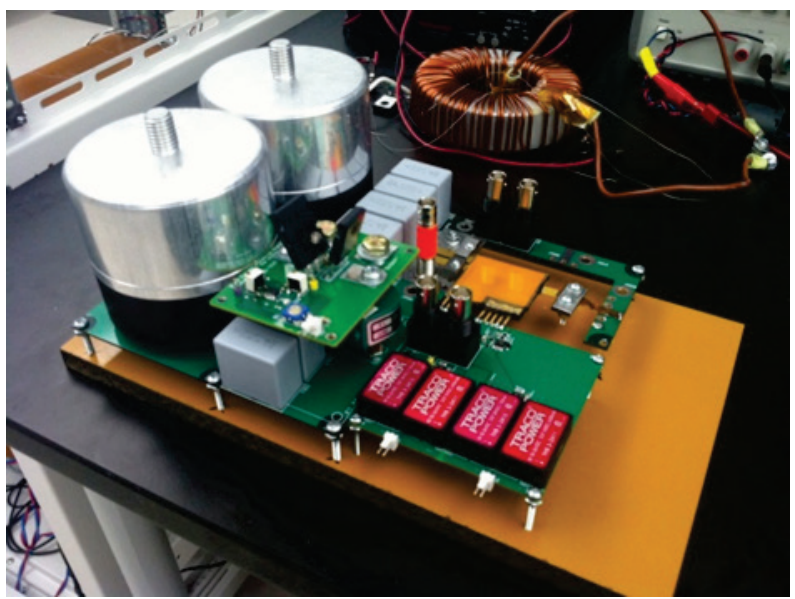

Figure 13: Photo of an experimental setup for evaluation of PBA SiC module electrical performance. 


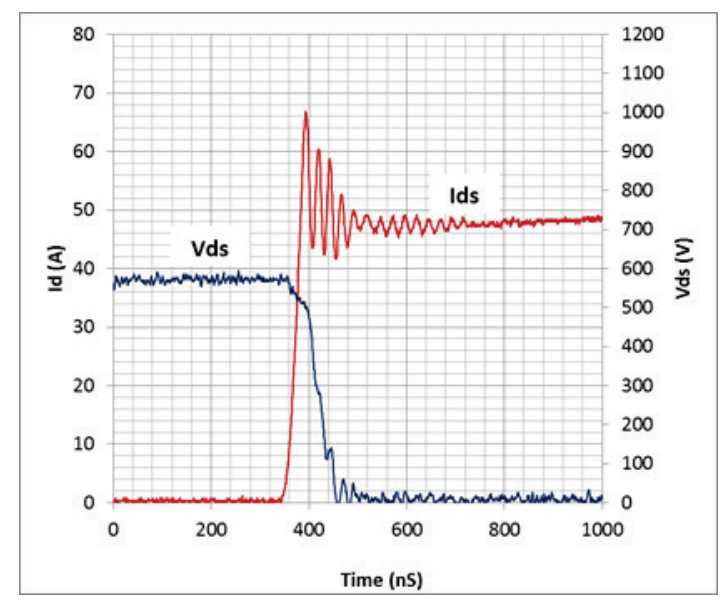

(a)

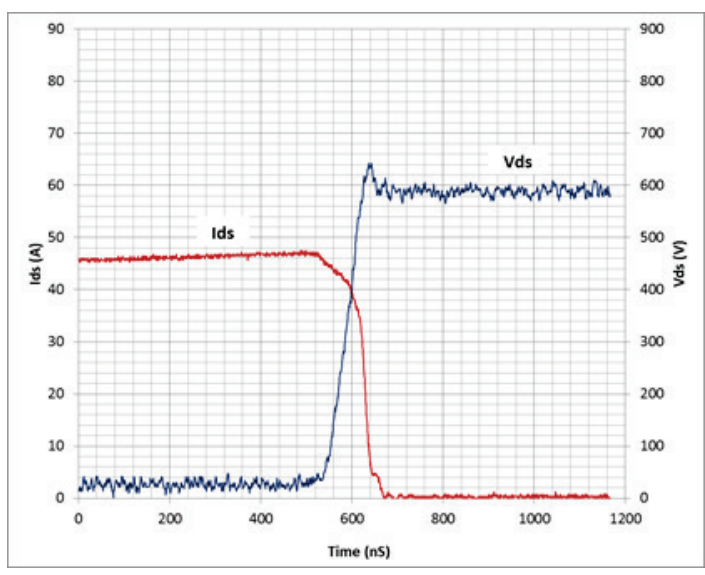

(b)

Figure 14: Current/voltage waveforms of PBA SiC module: (a) switching on and (b) switching off.

The thermal performance of the $\mathrm{SiC}$ power module was characterized by specific thermal resistance, $\theta \mathrm{ja}, \mathrm{sp}$, which is a normalized parameter by die area (i.e., $\theta \mathrm{ja}, \mathrm{sp}=$ die area $\times$ thermal resistance). It is the sum of those from all thermal stacking elements, including the coolant, pin-fin substrate, DBC substrates, and SiC dies. The specific thermal resistance of the double-sided cooling of the PBA module assembly is $0.33 \mathrm{~cm}^{2} \cdot{ }^{\circ} \mathrm{C} / \mathrm{W}$, compared with the specific thermal resistance of a conventional module assembly of $0.54 \mathrm{~cm}^{2} \cdot{ }^{\circ} \mathrm{C} / \mathrm{W}-\mathrm{a} 39 \%$ reduction in thermal resistance.

The reduction in both parasitic power losses and thermal resistance helps increase the operational current (power) density in the $\mathrm{SiC}$ die. It has been demonstrated that the integrated double-sided-cooled $\mathrm{SiC}$ module packaging increases the current density of the $\mathrm{SiC}$ power device by $53 \%$.

The current density increase helps reduce the $\mathrm{SiC}$ die size and, in turn, the cost of the power electronics system.

\section{Ag-Sintered Die Attachment SiC Module Packaging}

$\mathrm{Ag}$-sintered $\mathrm{SiC}$ die attachment was investigated by prototyping a $\mathrm{SiC}$ diode totem-pole module. Figure 15 shows photos of some typical samples. Figure 15(a) shows $10 \mathrm{SiC}$ diode dies bonded onto five DBC substrates at different locations. The two DBC substrates on the left have Ag finishing, while the three on the right have gold $(\mathrm{Au})$ finishing. The different designs allow evaluation of the effect of the metallization and die layout on the quality of this special die attachment.

The modules were packaged using processes identical to those used in the solder die attachment module packaging. Figure 15(b) shows the six aluminum wires (diameters of 10 mil) bonded onto the top of each diode die. A zoomed-in image also shows the sintered $\mathrm{Ag}$ on the substrate. Finally, the terminals were mounted on the substrate, and the entire module was encapsulated by Si gel, as shown in Figure 15(c).

Comprehensive evaluations including microstructural analysis, thermomechanical evaluation, and electrical reliability are under way in collaboration with other projects within the VTO program [6.1, PE and EM Materials Support], the results of which will be provided in future reports. 


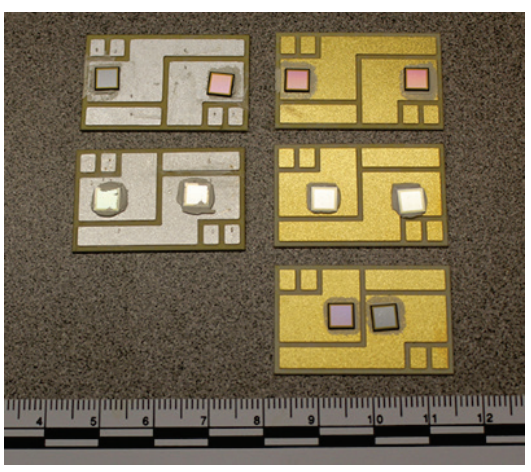

(a)

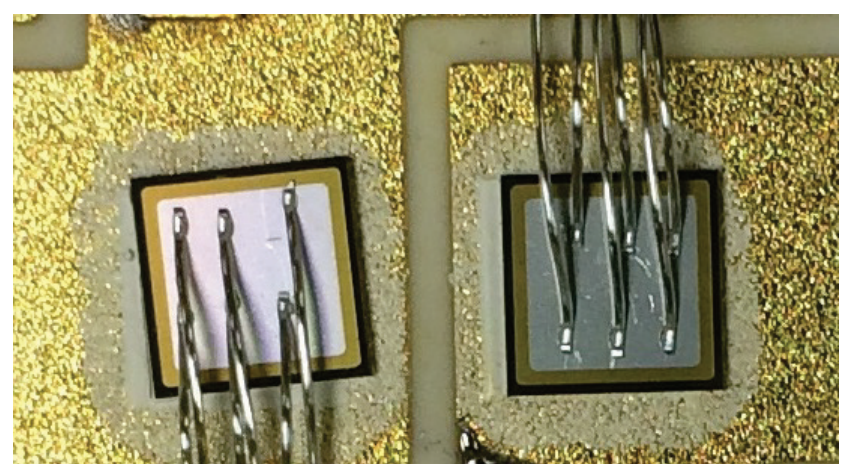

(b)

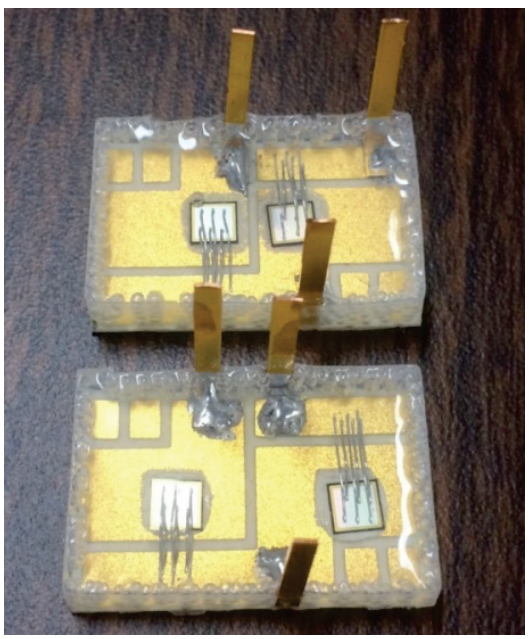

(c)

Figure 15: Photos of Ag-sintered die attachment SiC module packaging: (a) SiC diode dies bonded on DBC substrates, (b) zoomed-in die attachment and wire bonds, and (c) encapsulated power modules.

\section{Conclusions and Future Direction}

The advanced packaging technologies successfully developed in FY 2015 include packaging of SiC air-cooled inverter modules, integrated cooling packaging of planar power modules, and Ag-sintered bond power modules. The resulting improvements made in reducing electrical parasitics and thermal impedance enable the efficient exploitation of WBG power devices. The benefits gained from these innovations were demonstrated by comprehensive upgrades in $\mathrm{SiC}$ packaging performance, leading to high-efficiency, high-density system operation beyond the limits of state-of-the-art technologies. These advances resulted in higher power conversion efficiency (low losses) and improved cost-effectiveness through reductions in power semiconductor size and higher productivity in manufacturing.

Further advancement in WBG automotive power electronics will depend greatly on improvements in power packaging technology through advances in structure, materials, and processing techniques. The focus will be on developing highly integrated functionality for WBG power inverter/converter modules with intelligence and improved operating performance (efficiency, density, and cost). The effort will include (1) incorporating advanced gate drive circuitry into the package, (2) implementing highly integrated cooling technologies for integrated multiphase conversion systems, (3) optimizing interconnection layouts and embedding passives for electromagnetic interference containment and sensors, and (4) developing processes for temperature-tolerant integrated $\mathrm{SiC}$ power module packages.

These advancements will enable considerable strides to be made toward achieving DOE power density and cost targets for power electronics systems in electric drive vehicles. 


\section{Publications/Presentations}

1. Z. Wang, X. Shi, L. M. Tolbert, Fellow, F. Wang, Z. Liang, D. Costinett, and B. J. Blalock, “A high temperature silicon carbide MOSFET power module with integrated silicon-on-insulator-based gate drive," IEEE Transactions on Power Electronics 30(3), 1432-1445, March 2015.

2. A. A. Wereszczak, Z. Liang, M. K. Ferber, and L. D. Marlino, "Uniqueness and challenges of sintered silver as a bonded interface material," Journal of Microelectronics and Electronic Packaging 11, 158-165, 2014.

3. Z. Liang, "Integrated double sided cooling packaging of planar SiC power modules," The Seventh Annual IEEE Energy Conversion Congress and Exposition (ECCE 2015), Montreal, Canada, September 20-24, 2015.

4. Z. Liang, "Advanced packaging technologies for fully exploiting attributes of WBG power electronics,” IEEE International Workshop on Integrated Power Packaging (IEEE IWIPP'15), Chicago, Illinois, May 3-6, 2015.

5. Z. Liang, "Planar-bond-all: A technology for three-dimensional integration of multiple packaging functions into advanced power modules," IEEE International Workshop on Integrated Power Packaging (IEEE IWIPP'15), Chicago, Illinois, May 3-6, 2015.

6. Z. Wang, X. Shi, L. M. Tolbert, F. Wang, Z. Liang, D. Costinett, and B. J. Blalock, "Development of a board-level integrated silicon carbide MOSFET power module for high temperature application," IEEE International Workshop on Integrated Power Packaging (IEEE IWIPP'15), Chicago, Illinois, May 3-6, 2015.

7. Z. Liang, "Development of packaging technologies for advanced $\mathrm{SiC}$ power modules," the Second IEEE Workshop on Wide Bandgap Devices and Applications (WiPDA), Knoxville, Tennessee, October 13-15, 2014.

8. Z. Liang, "Power electronics packaging," DOE AMR, Washington, DC, June 10, 2015.

9. Z. Liang, "Advanced packaging technologies and designs," Kick-off meeting of DOE Vehicle Technologies Office-Electric Drive Technologies, Oak Ridge, Tennessee, November 19, 2014.

10. Z. Liang, "Packaging technologies to exploit the attributes of WBG power electronics," tutorial presented at the Second IEEE Workshop on Wide Bandgap Devices and Applications (WiPDA), Knoxville, Tennessee, October 13-15, 2014.

11. Z. Liang, "Packaging technology for multi-functional integration of advanced SiC power modules," presented at the Fourth International Power Supply on Chip Workshop (PwrSoC2014), Boston, Massachusetts, October 6-8, 2014.

\section{Patents}

12. Z. Liang, "Integrated Packaging of Multiple Double Sided Cooling Planar Bond Power Modules," provisional patent application 62/167,371 filed for ID-3211, May 28, 2015.

13. Z. Liang, P. Ning, F. Wang, and L. Marlino, "Power Module Packaging with Double Sided Planar Interconnection and Heat Exchangers," US Patent No. 9,041,183, May 25, 2015. 


\title{
6.1 Power Electronics and Electric Motor Materials Support (Joint with VTO Propulsion Materials)
}

\author{
Andrew Wereszczak, Principal Investigator \\ Oak Ridge National Laboratory (ORNL) \\ National Transportation Research Center \\ 2360 Cherahala Boulevard \\ Knoxville, TN 37932 \\ Phone: (865) 946-1543 \\ E-mail: wereszczakaa@ornl.gov
}

Susan A. Rogers, DOE EDT Program Manager

Phone: (202) 586-8997

E-mail: Susan.Rogers@ee.doe.gov

Burak Ozpineci, ORNL EDT Program Manager

Phone: (865) 946-1329

E-mail: burak@ornl.gov

Contractor: UT-Battelle, LLC, managing and operating contractor for the Oak Ridge National Laboratory Contract No.: DE-AC05-00OR22725

\section{Abstract/Executive Summary}

- Materials research and development (R\&D) support is provided to both power electronics (PE) and electric motor (EM) research efforts under way at the National Transportation Research Center via joint funding from the VTO Propulsion Materials and Electric Drive Technologies (EDT) Programs.

- PE support: Most FY 2015 effort involved cross-cutting applied R\&D of sinterable silver (Ag) as a candidate interconnect for PE devices. Sintered-Ag interconnection technology has several advantages over conventionally used solder-based interconnection technology (e.g., better electrical and thermal conductivity, microstructural equilibrium, potential for much better reliability), but adoption has been slow because it is a relatively new technology and the PE community is relatively conservative. This effort attempts to hasten the more confident adoption of sintered-Ag technology and consequent improvement in the reliability of automotive PE devices. Work in FY 2015 focused on understanding the concurrent mechanical limitations of sinterable $\mathrm{Ag}$ and the plating layers that interface it to substrates and die. PE-materials R\&D support was also provided to EDT Tasks 4.1 (Benchmarking EV and HEVs, Burress, PI) and 5.1 (Power Electronic Packaging, Liang, PI). Portions of the PE materials effort involved an ORNL-led collaboration with the National Renewable Energy Laboratory (NREL).

- $\quad$ EM support: Two primary applied R\&D efforts occurred in FY 2015 that supported EDT Task 2.1 (Non-Rare Earth Motor Development, Burress, PI). The first effort involved the investigation of new candidate potting compounds and molding compounds that could have better thermal transfer characteristics and higher-temperature capability and that are economically competitive. The second effort involved a fundamental study and measurement of the directional thermal transfer in copper windings used in EMs; it involved an ORNL-led collaboration with NREL. Both efforts will ultimately enable more efficient automotive EM operation and smaller and lighter EMs.

\section{Accomplishments}

- PE support

- Quantified the shear strength of sintered-Ag interconnects as a function of printing method (screen vs. stencil printing) and plating material (Ag vs. gold or $\mathrm{Au}$ ). Initiated failure analysis to censor the measured strengths in the context of cohesive and adhesive failure locations. These results will illustrate the achievable strength characteristics of such interconnect systems, guide 
future sintered-Ag design for PE, and improve the community's receptiveness to adopting sintered-Ag technology.

- Conceived of, coordinated, fabricated all specimens for, and assumed leadership of an ORNL-led collaboration with NREL to study delamination initiation and propagation response in sintered-Ag interconnects.

- Sectioned and analyzed the micro-architecture of the power module used in the Honda Accord inverter in support of EDT Task 4.1.

- Used sintered $\mathrm{Ag}$ as an interconnect to bond an $\mathrm{SiC}$ die to direct-bonded copper (DBC) substrates in support of EDT Task 5.1.

- Fabricated sintered-Ag samples for thermal and mechanical testing at NREL (DeVoto).

- $\quad$ EM support ( EDT Task 2.1)

- Measured thermal properties of developmental, mineral-filled potting and molding compounds and established an collaboration with an industrial manufacturer of such materials.

- Conceived of, coordinated, fabricated all specimens for, and assumed leadership of an ORNL-led collaboration with NREL to better understand the directional thermal response of copper-wound structures.

- Developed versatile copper-wound billets from which samples were harvested to enable the thermal property measurement of their anisotropic structure using three different standardized techniques.

- Showed that the thermal conductivity in the direction of the copper wires is over two orders of magnitude higher than the thermal conductivity perpendicular to them.

\section{Introduction}

For future PE devices, the potential use of sintered-Ag interconnection technology has several advantages over conventionally used solder-based interconnection technology (e.g., better electrical and thermal conductivity, microstructural equilibrium, potential for much better reliability). However, its adoption has been slow because it is a relatively new technology and the PE community is relatively conservative. This VTO Propulsion Materials-EDT jointly funded effort will hasten the more confident adoption of sintered Ag by identifying the achievable strength characteristics of such interconnect systems (which are relative complex, as illustrated in Figure 1). This effort will guide future sintered-Ag design for PE and improve the PE community's receptiveness to adopting sintered-Ag technology.

For EMs, minimizing the service temperature in copper windings in slot liners will promote greater efficiency and enable EM size and weight reductions. Figure 2 illustrates these wires in slot liners. Two ways to achieve minimization are by using more thermally conductive materials in EMs and by improving the understanding of the anisotropic thermal transfer within those copper windings. New potting and molding compounds that have better thermal transfer characteristics and higher-temperature capability are attractive candidates for this purpose, provided they are cost-competitive with currently used materials and do not introduce performance compromises. Greater understanding of the thermal transfer characteristics starts with the fundamental study and measurement of the directional thermal transfer in copper windings used in EMs; however, specimens must be developed and thermally measured that represent how these copper windings thermally respond in service. 


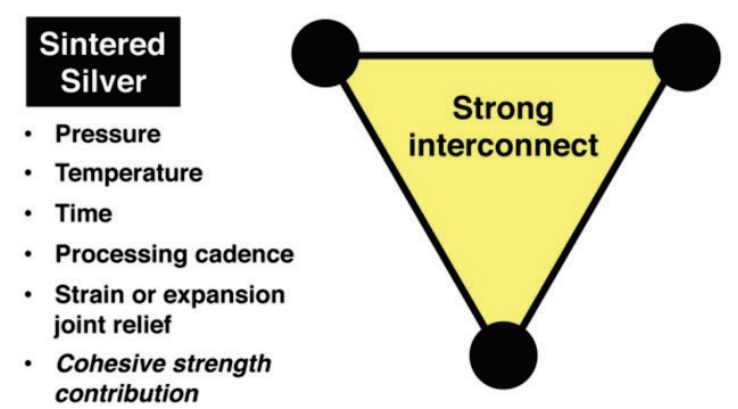

Plating

- Thickness

- Cleanliness

- O, C, and S contamination

- Promote metallurgical bond

- Chemical component of adhesive strength

Die, Substrate, or Baseplate

- Surface finish \& topography

- CTE (mismatch)

- Size/area

- Mechanical component of adhesive strength

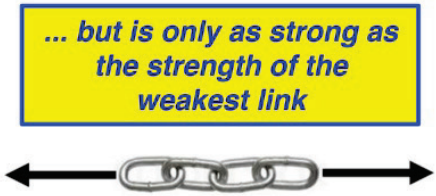

Figure 1: The strength and reliability of a sintered-Ag interconnect is a function of many parameters.

Figure 2: Example of copper windings in slot liners [Sato et al., SAE Intl, 2011].

\section{Approach}

\section{Power Electronics Support}

Fabricate custom-designed sintered-Ag test coupons that enable the measurement of shear strength as a function of the plating material (Ag vs. $\mathrm{Au}$ ) and printing method (screen vs. stencil). Perform failure analysis to identify location in the context of either cohesive or adhesive failure of any of the constituents making up the sintered-Ag interconnect system.

Coordinate and lead an ORNL-led sintered-Ag thermomechanical reliability study with NREL. Examine the effect of coefficient-of-thermal-expansion (CTE) -induced residual stress on sintered-Ag printed pad size and the onset of delamination of the interconnect. Fabricate all specimens and provide materials characterization.

Section, metallographically polish, and analyze the micro-architecture of the power module used in the Honda Accord inverter in support of EDT Task 4.1. The analyses included optical microscopy, scanning electron microscopy, and chemistry mapping via energy-dispersive spectroscopy.

Bond an SiC die to DBC substrates using sintered Ag in support of EDT Task 5.1. Bond the die at different locations to ready it for different wire bonding placements and eventual device testing.

Fabricate sintered-Ag samples for NREL's internal thermal and mechanical evaluations (DeVoto). 


\section{Electric Motor Support}

Support EDT Task 2.1 and measure thermal properties of developmental, mineral-filled potting and molding compounds.

Coordinate and lead an ORNL-led collaboration with NREL (Bennion) to better understand the directional thermal response of copper-wound structures. Conceive of and fabricate test specimens that facilitate the valid measurement of thermal diffusivity and thermal conductivity as a function of wire orientation. Use different methods of thermal property measurement to enable comparisons and validate the legitimacy of the results.

\section{Results and Discussion}

\section{Power Electronics Support}

Custom-designed sintered-Ag test coupons were fabricated that enabled the measurement of shear strength as a function of plating material (Ag vs. Au) and printing method (screen vs. stencil). A scanning acoustic microscopy image of some of these samples is shown in Figure 3. Polished cross-sections of the platings are shown in Figure 4. Silver was plated directly onto the copper cladding on the DBC substrate, but an Ni-P interphase was applied onto the copper. Then the Au plating was deposited onto the Ni-P. The squares were then shear-tested to failure using a Nordson-Date tester (see Figure 5), and their uncensored results were regressed against a 2-parameter Weibull distribution (graphically shown in Figure 6).

Failure analysis is occurring in early FY 2016 to link each specimen's strength with failure locations (i.e., censor the data). This data censoring will provide context to either cohesive or adhesive failure of any of the constituents making up the sintered-Ag interconnect system.

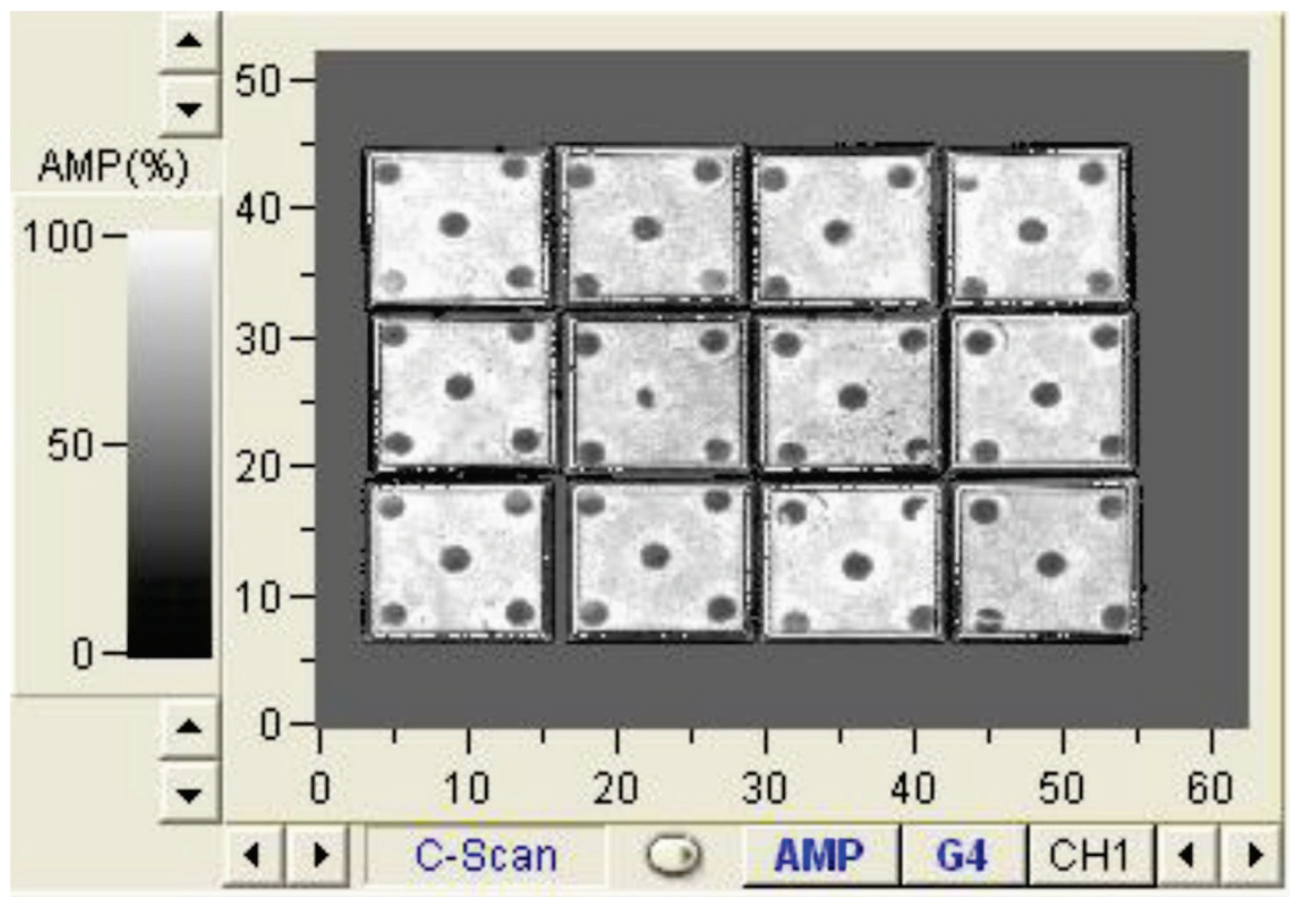

Figure 3: Scanning acoustic microscopy image of the "5-dice-pattern" interconnect used with DBC sandwiches to quantify shear strength. Squares $(12.7 \times 12.7 \mathrm{~mm})$ are the DBC substrates. The dark circles are the sintered-Ag interconnecting pads. Total bonds are nominally $20 \mathrm{~mm}^{2}$. 

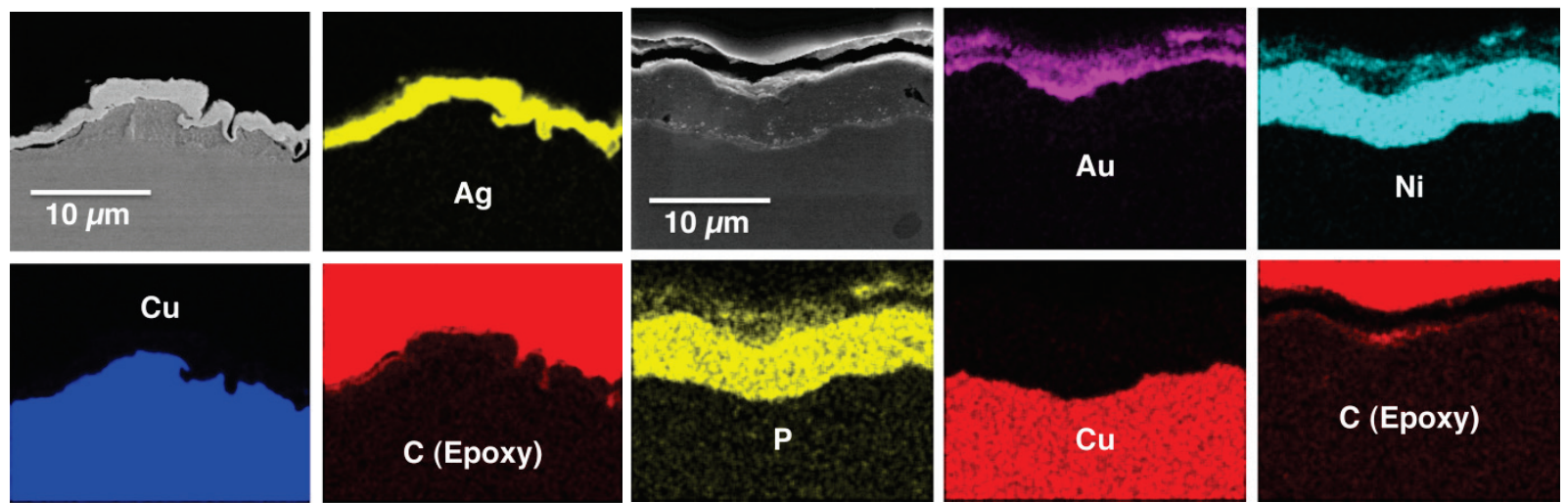

Figure 4: Polished cross-sections of the silver (left) and gold (right) platings used on the DBC substrates.

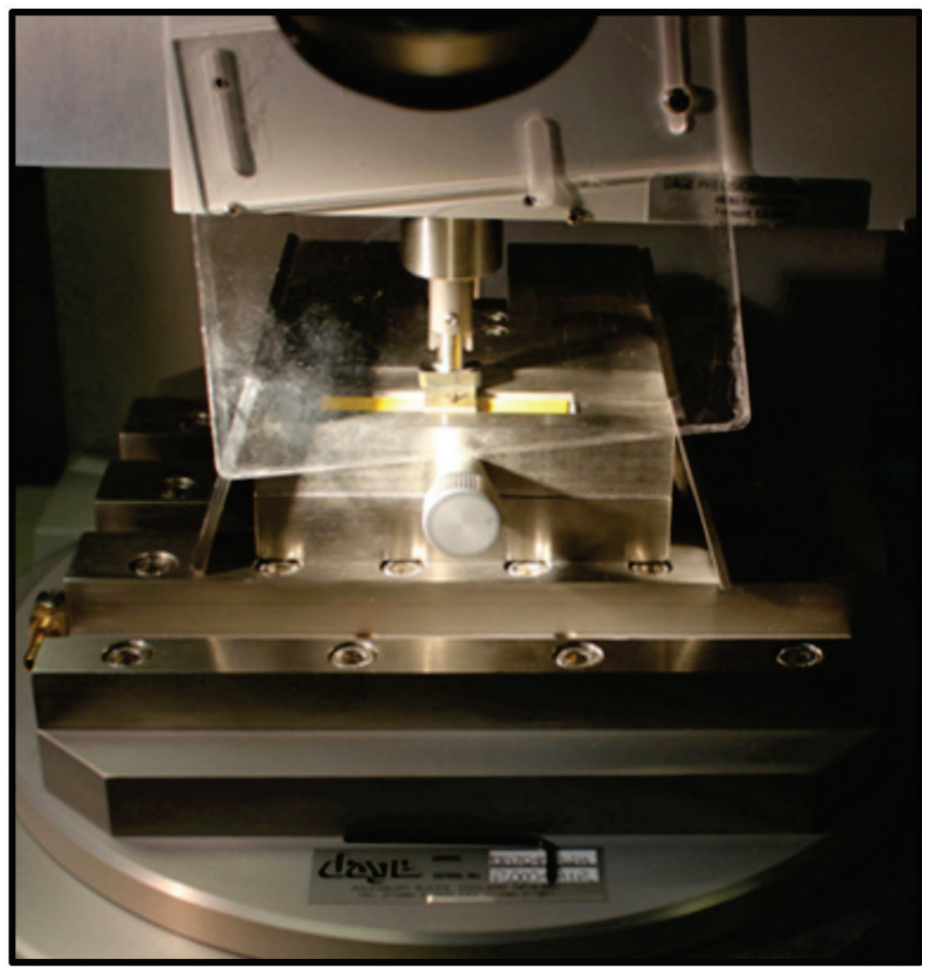

Figure 5: Test setup for measuring the shear strength of the DBC sandwiches.

ORNL conceived of and is leading a collaboration with NREL (DeVoto) involving the study of the thermomechanical response of sintered $\mathrm{Ag}$ as a function of CTE-mismatch-induced residual stress. Residual stress was purposely introduced and varied using the conditions described in Figure 7. An "Oreo-cookie" sample was chosen because of its axisymmetric simplicity and to avoid complications introduced by the presence of corners. Invar (metal) was chosen for this study because its CTE is similar to that of silicon, and because the weakness and brittleness of silicon can prohibit measurement of the shear strength of the interconnect material. Three different sintered-Ag pad diameters and three different combinations of $\mathrm{Cu}-\mathrm{Cu}$, Invar-Invar, and $\mathrm{Cu}$-Invar were used, producing the processed matrix shown in Figure 8. These disks are now being thermally cycled at NREL to monitor the onset and rate of advance of delamination of the sintered-Ag interconnect. 


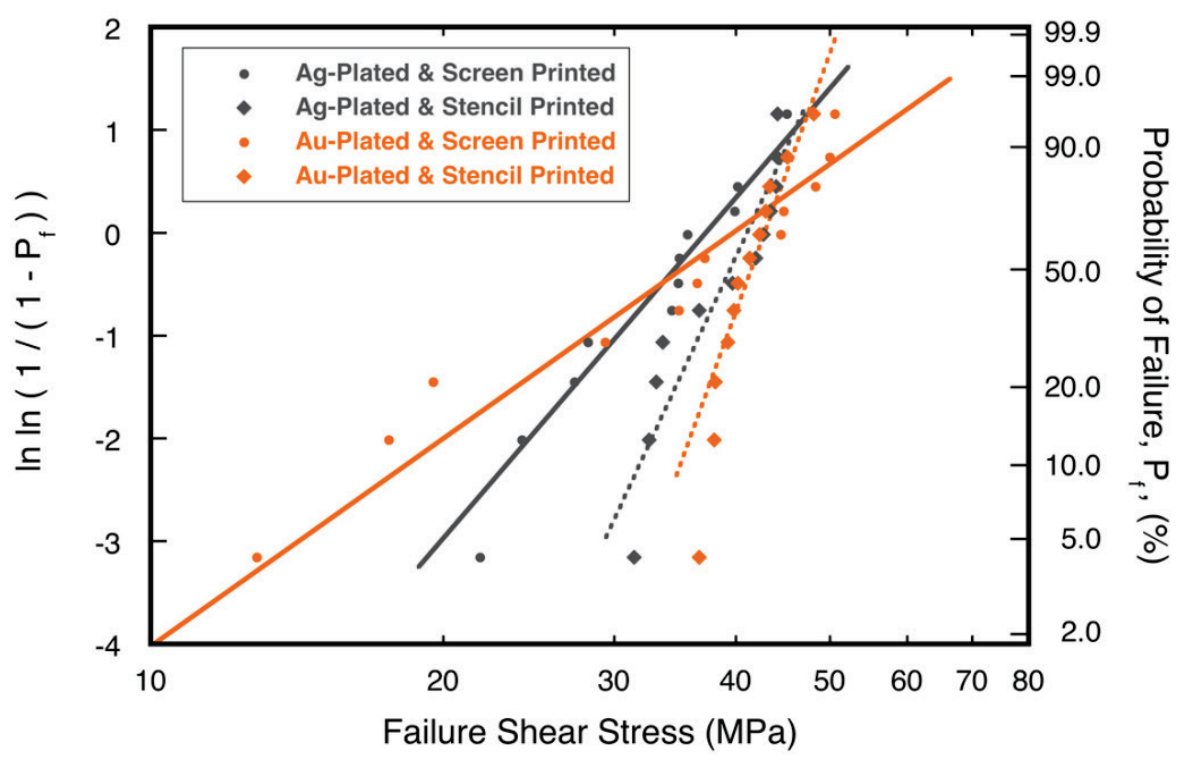

Figure 6: Failure shear stress values fitted against 2-parameter Weibull distributions. Specimens are now undergoing failure analysis and data censoring.

\section{Side View}

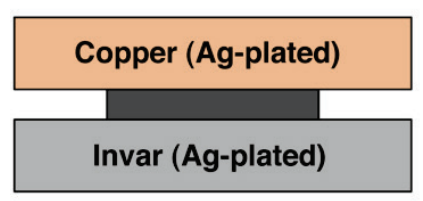

- Invar: model material used to simulate low CTE of silicon and substrates in electronic devices

- Vary pad diameter of bond layer

- Vary residual stress

- Copper-copper disk pair

- Invar-invar disk pair

- Copper-invar disk pair

- Thermal cycling $\left(-40^{\circ} \mathrm{C}\right.$ to $\left.140^{\circ} \mathrm{C}\right)$ $\&$ track delamination response

Independent Parameters: Magnitude of residual stress Orientation of residual stress

\section{Top View}

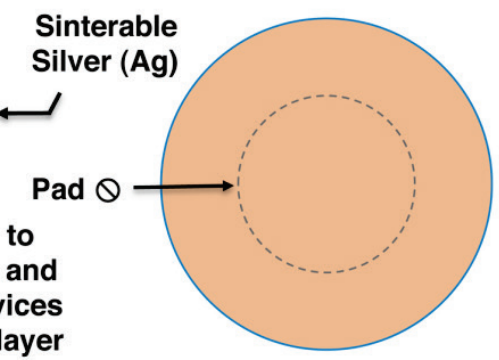

Nominal Properties:

\begin{tabular}{|c|c|c|}
\hline Material & $\mathrm{CTE}\left(\mathrm{ppm} /{ }^{\circ} \mathrm{C}\right)$ & $\mathrm{E}(\mathrm{GPa})$ \\
\hline Copper & 17 & 115 \\
\hline Sintered $\mathbf{A g}$ & 20 & $15-60$ \\
\hline Invar & $1.3-2.7$ & 145 \\
\hline
\end{tabular}

Figure 7: Description of "Oreo-cookie" test coupons used to study the effect of CTE-induced residual stress on delamination onset and propagation. Sintered-Ag print diameters are 10, 18, and $22 \mathrm{~mm}$ on these $25 \mathrm{~mm}$ diameter disks of either copper or Invar.

A delamination effect was immediately observed after the processing of these disks. Its analysis is ongoing with the intent of advocating a simple method to identify the potential maximum bond size of a sintered-Ag interconnect for a given suite of independent parameters (like those illustrated in Figure 1). Scanning acoustic microscopy showed that some of the interconnects had started to delaminate as a consequence of the hightemperature "cookie" sintering (Figure 9). The analysis will be described in a manuscript to be submitted to the open literature with NREL as a collaborator. 


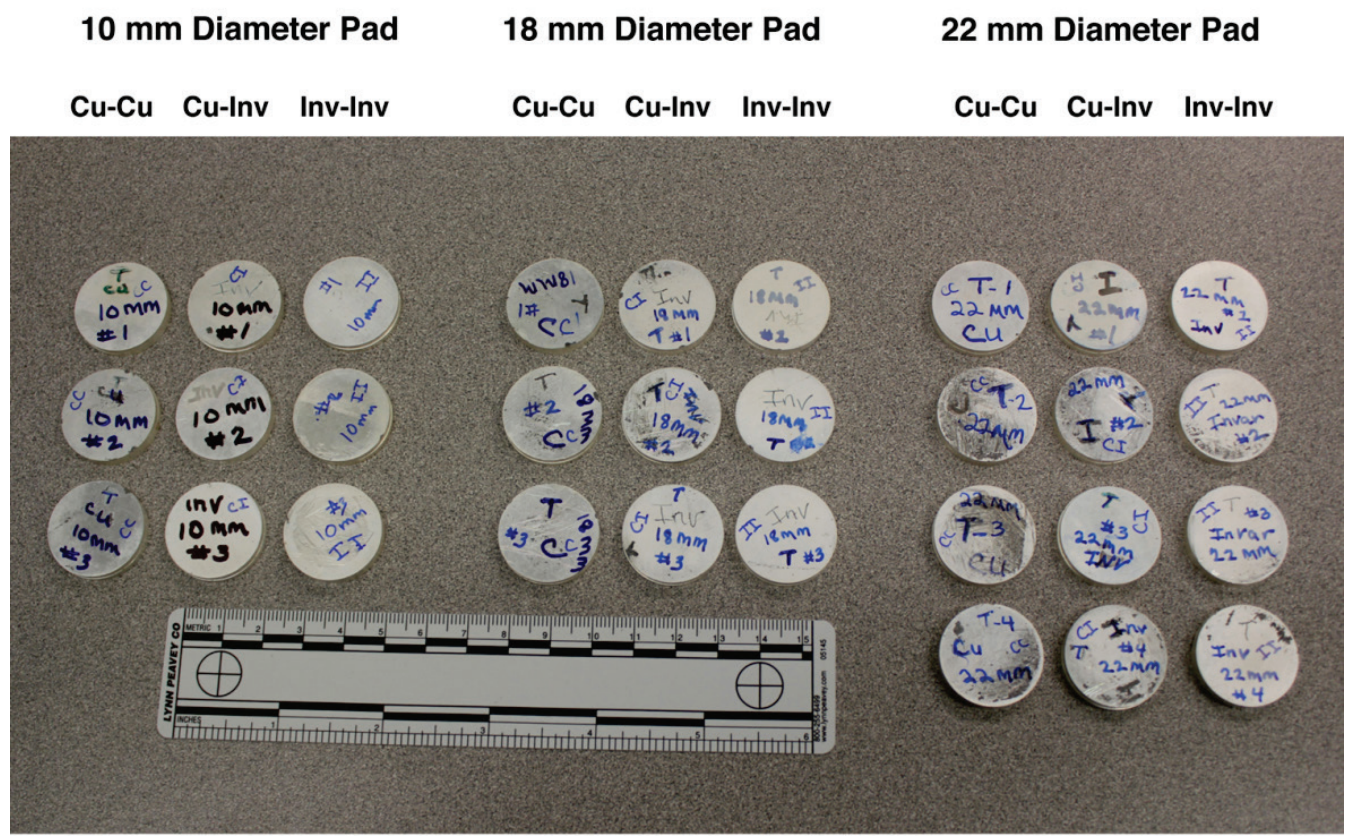

Figure 8: Matrix of "cookie" samples for the three sintered-Ag bond diameters and three different combinations of copper and invar. At least three samples were made of each.

Example: Copper-Invar 22-mm Print Diameter

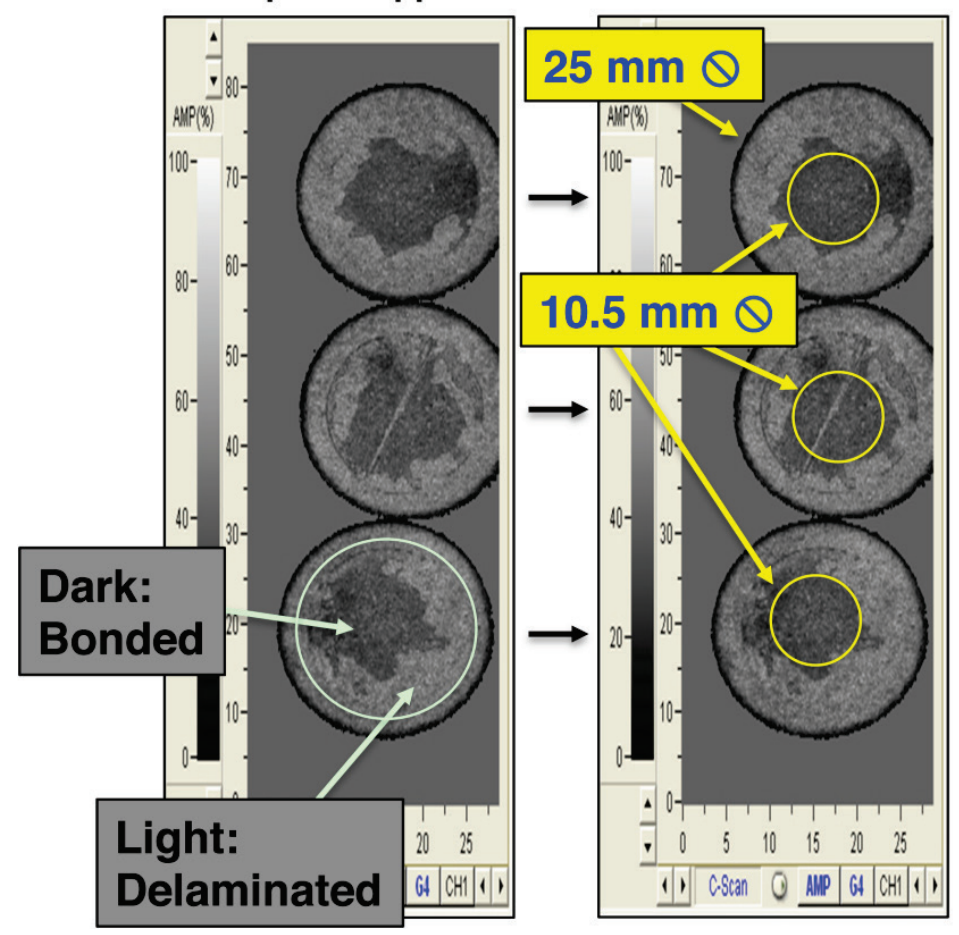

Figure 9: Preliminary scanning acoustic microscopy shows a residual bond area/size, which is being analyzed as a way to identify the maximum allowable sintered-Ag interconnect size.

A power module was sectioned and metallographically polished (Figure 10) to examine its micro-architecture (Figure 11). Additional details of this work are featured in EDT Task 4.1 reporting and are not repeated here. 


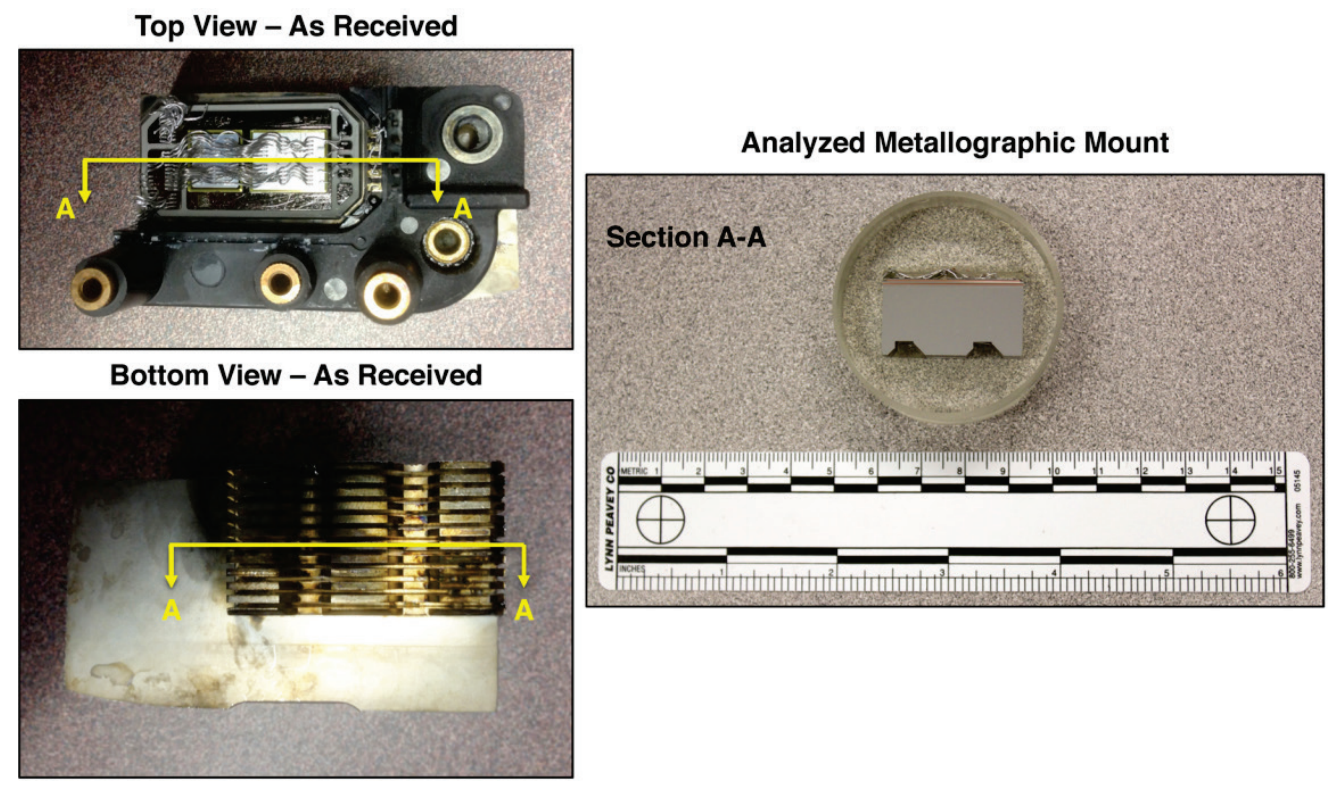

Figure 10: The power module from a Honda Accord was sectioned and metallographically prepared.

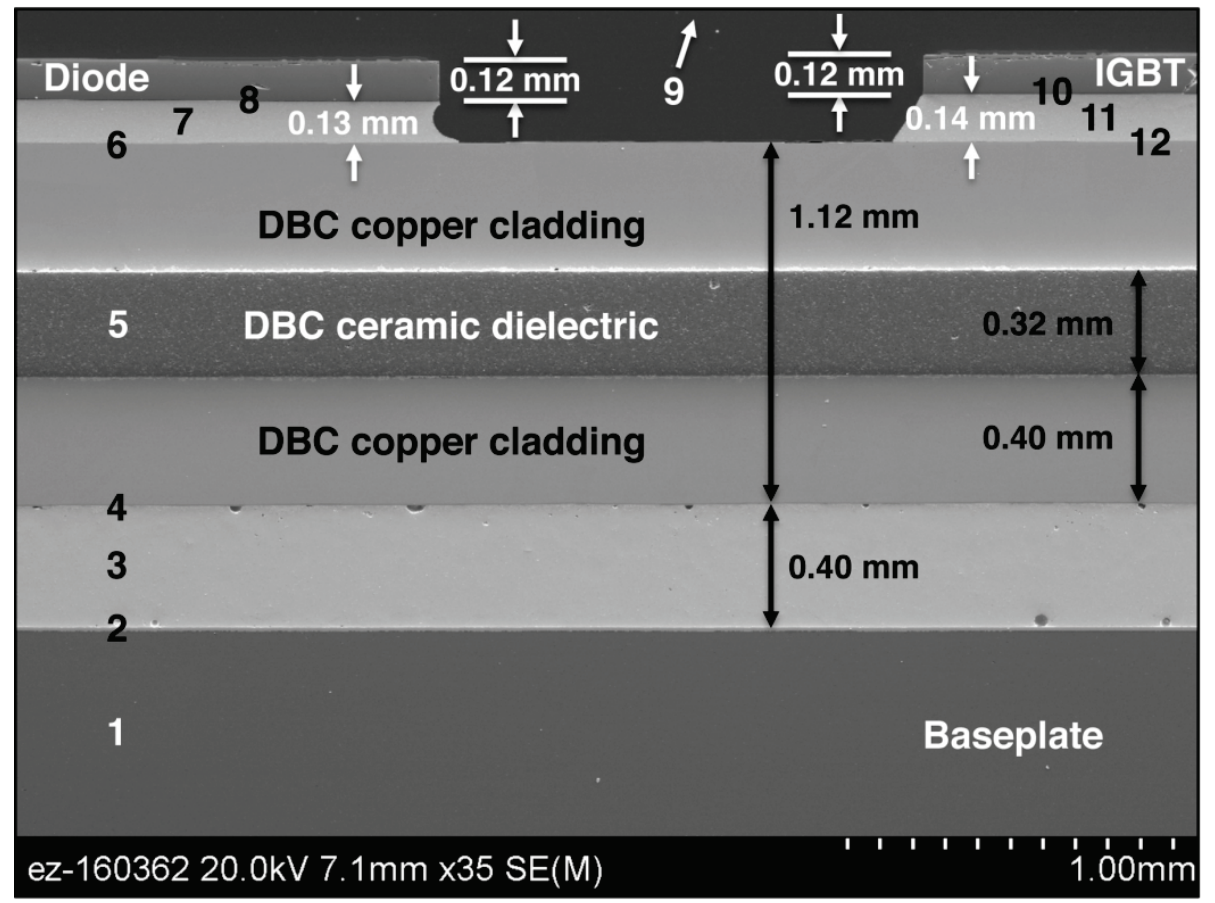
1. Baseplate
2. Baseplate plating
3. Solder - Baseplate/DBC
4. DBC plating
5. Ceramic in DBC
6. DBC plating
7. Solder - DBC/Diode
8. Diode plating
9. Wire (not shown)
10. IGBT plating
11. Solder - DBC/IGBT
12. DBC plating (same as \#6)

Figure 11: Annotated cross-section of power module shown in Figure 10.

In support of EDT Task 5.1, SiC dies were bonded to Ag- and Au-plated DBC substrates to ready them for wire bonding placement and eventual device testing (Figure 12). 


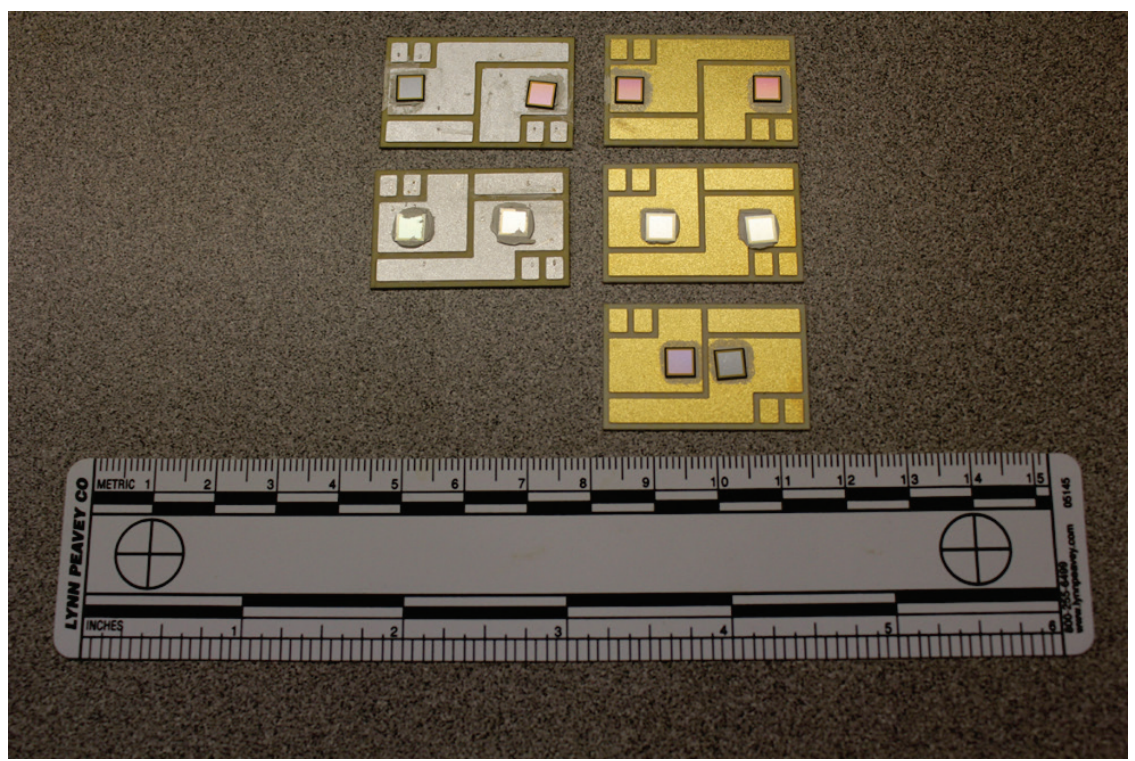

Figure 12: Examples of preliminary sintered-Ag bonding of SiC to either Ag-plated or gold-plated DBC substrates.

Two additional sintered-Ag processing efforts were undertaken for the benefit of NREL (DeVoto). They included the sintered-Ag bonding of silicon disks to metal disks (Figure 13) for NREL's internal thermoreflectance measurements and sintered-Ag bonding of squares to make double-lap shear specimens for mechanical testing (Figure 14). ORNL designed and fabricated the necessary fixtures to promote concentric alignment of the bonded pieces and then executed the sintered-Ag bonding.

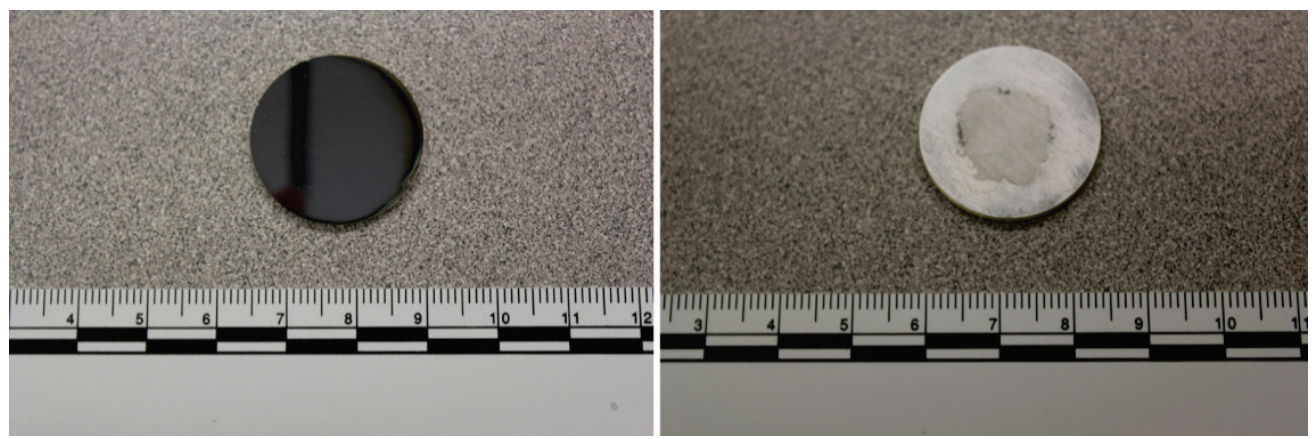

Figure 13: Disk specimens were sintered-Ag bonded by ORNL for NREL for internal thermoreflectance measurements.

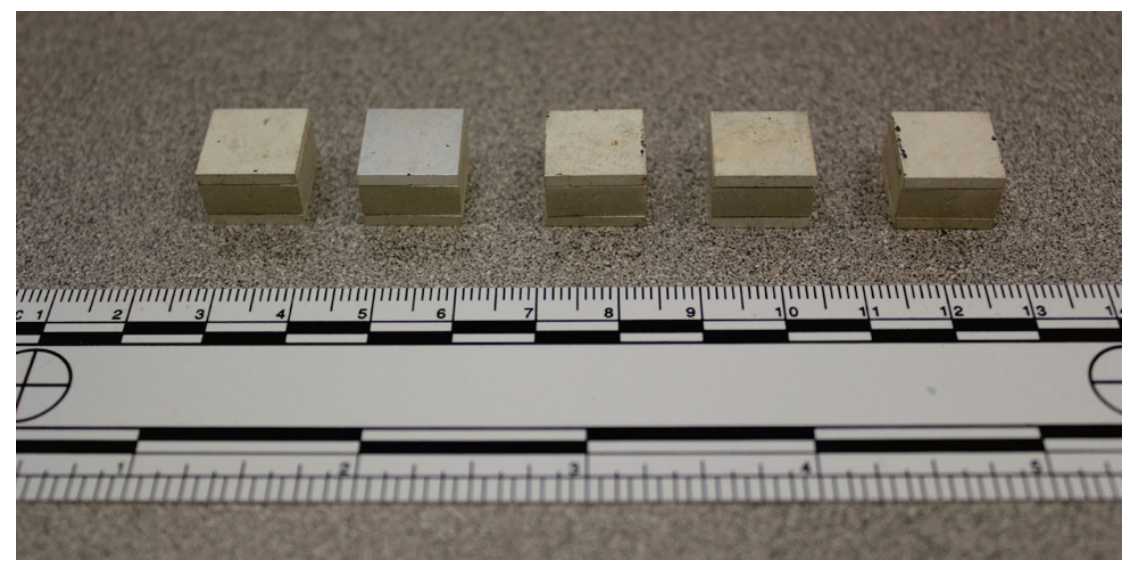

Figure 14: Double-lap specimens were sintered-Ag bonded by ORNL for NREL to conduct shear tests. 


\section{Electric Motor Support}

Thermal property measurements were made with a wide variety of mineral-filled molding compounds (example shown in Figure 15). Additional work and refinement, and a new collaboration with an established molding compound manufacturer, will continue in FY 2016. This effort is a subtask to EDT Task 5.1.

Also as part of EDT Task 5.1, ORNL conceived of, coordinated, and led a collaboration with NREL (Bennion) to better understand the directional thermal response of packed copper wires. The effort included the examination of valid measurements of thermal diffusivity and thermal conductivity as a function of wire orientation. Six cubes of copper windings (nominally $50 \mathrm{~mm}$ on a side) (Figure 16) were fabricated at ORNL using conventional varnish with custom-fabricated molds. Three were fabricated with 19 gage wire and three with 22 gage wire. Polished cross sections of these copper wires are shown in Figure 17. Laser flash diffusivity (Figure 18) conclusively and quantitatively showed that the thermal conductivity in the direction parallel to the copper wires was more than 2 orders of magnitude higher than the thermal conductivity perpendicular to the wires. The combination of the varnish, interstitial voids, and wire coatings all combined to compromise thermal transfer perpendicular to the wires. These results will be discussed in a journal article, along with values to be measured by a conduction method at NREL, and the work will continue in FY 2016. Additional details of this work are featured in Task 5.1 reporting and are not repeated here.

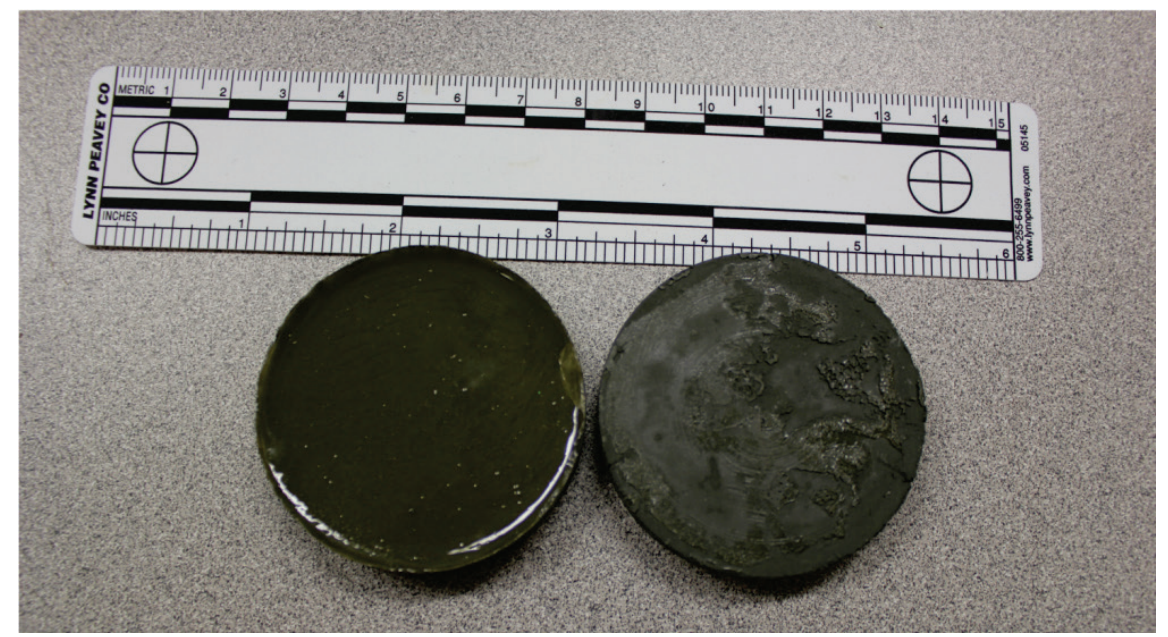

Figure 15: Examples of mineral-filled molding compounds fabricated at ORNL.

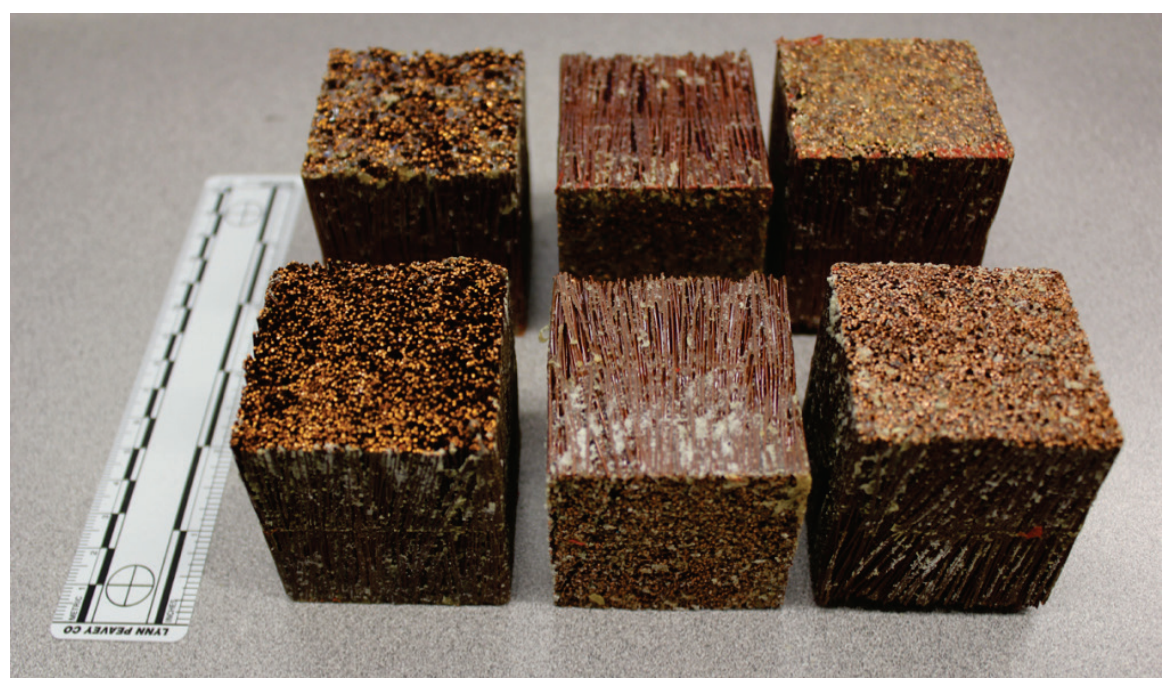

Figure 16: Custom copper-wound specimens were fabricated to facilitate the measurement of directional thermal diffusivity and thermal conductivity. Two copper wire diameters also were examined. 

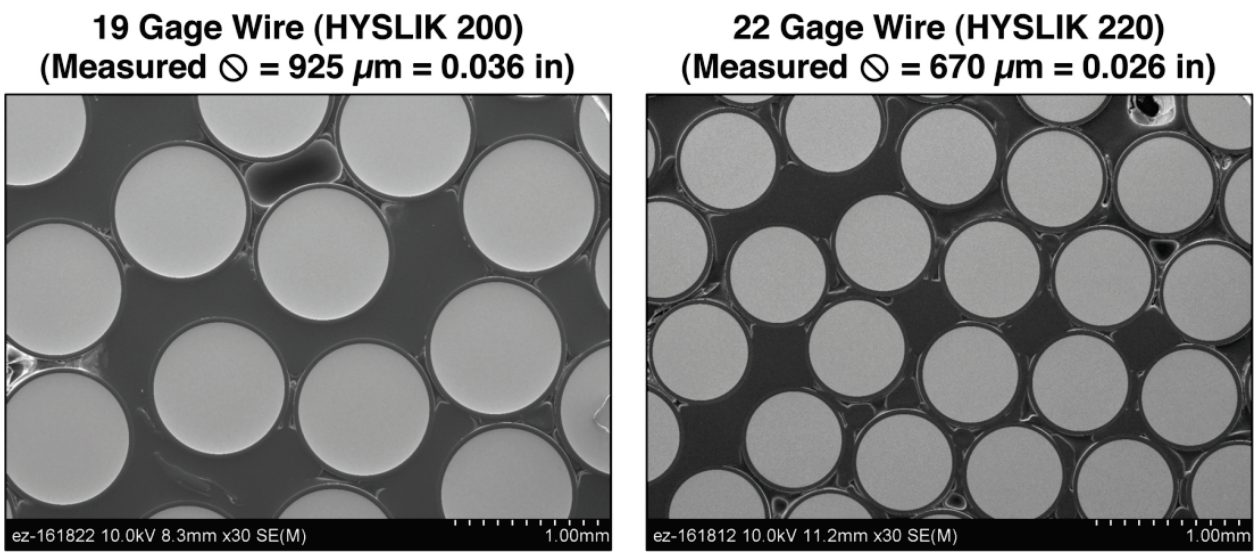

Figure 17: Polished cross sections of the two copper wire diameters used in the specimens shown in Figure 16.

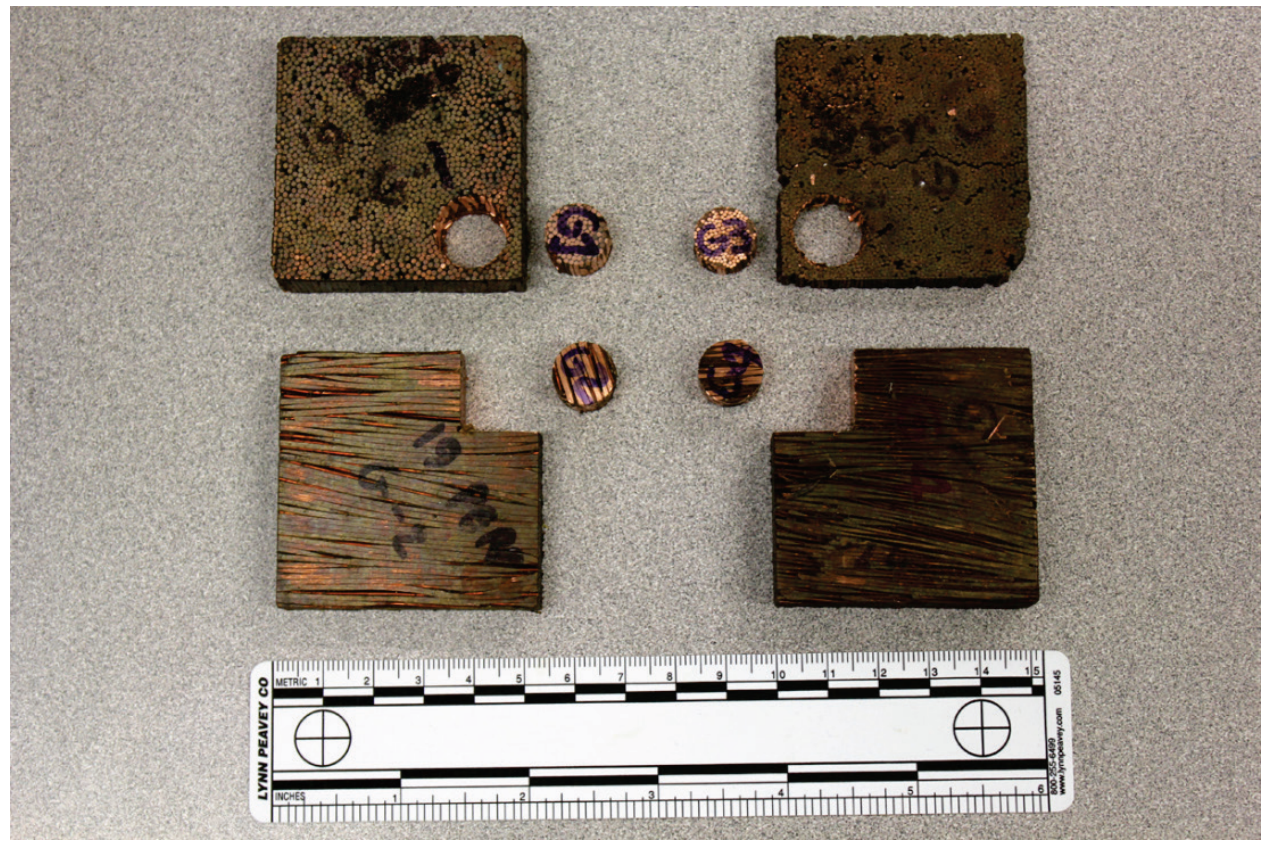

Figure 18: Core-drilled specimens were harvested from thin slabs of packed copper wire and used for thermal diffusivity measurements.

\section{Conclusions and Future Direction}

The shear strengths of sintered-Ag interconnect systems were on the order of 40-50 MPa. Preliminary failure analysis showed the concurrency of different failure mechanisms that limit those strengths. It also indicated that PE devices having sintered-Ag interconnects should be designed so that the maximum service stress is some fraction (safety factor) of the measured shear stress. Additional sintered-Ag efforts are planned for FY 2016 that will further increase the versatility of the processing and promote receptiveness to this technology.

There appears to be a simple method of identifying the maximum allowable sintered-Ag bond size. Work in FY 2016 will enable a more complete understanding of this activity in the context of thermal cycling.

Laser flash testing showed that the thermal conductivity in the direction of the copper wires is over 2 orders of magnitude higher than the thermal conductivity perpendicular to them. Now that these reference thermal property values are available for these fundamentally simple architectures, our attention in FY 2016 will turn 
toward (1) how to improve the thermal transfer perpendicular to the wires and (2) understanding thermal property variability in the context of wire diameter.

\section{FY 2015 Presentations/Publications/Patents}

1. A. A. Wereszczak, Z. Liang, M. K. Ferber, and L. D. Marlino, "Uniqueness and challenges of sintered silver as a bonded interface material," Journal of Microelectronics and Electronic Packaging 11,158-165 (2014).

2. A. A. Wereszczak, S. B. Waters, and W. Carty, "Transfer Method for Printed Sinterable Paste Having Nonaqueous Solvent," Invention Disclosure Number 201503508, DOE S-138,140, April 4, 2015.

3. A. A. Wereszczak, and W. Carty, "Drying Method for Sinterable Paste Used for Bonded Joints," Invention Disclosure Number 201503507, DOE S-138,139, April 3, 2015.

4. A. A. Wereszczak, Z. Liang, and T. A. Burress, "Enabling materials for high-temperature power electronics," presented at the 2015 VTO AMR, Arlington, Virginia, June 10, 2015.

5. A. A. Wereszczak, S. B. Waters, M. Modugno, D. J. DeVoto, and P. P. Paret, "Method to determine maximum allowable sinterable silver interconnect size," in preparation, 2015. 